\title{
O FLUXO DE POTÊNCIA ÓTIMO REATIVO COM \\ VARIÁVEIS DE CONTROLE DISCRETAS E RESTRIÇÕES DE ATUAÇÃO DE DISPOSITIVOS DE CONTROLE DE TENSÃO
}

Tese apresentada à Escola de Engenharia de São Carlos da Universidade de São Paulo como parte dos requisitos para obtenção do título de Doutor em Ciências, Programa de Engenharia Elétrica.

Área de Concentração: Sistemas Elétricos de Potência.

Orientador: Prof. Dr. Geraldo Roberto Martins da Costa.

Coorientadora: Profa. Dra. Vanusa Alves de Sousa.

São Carlos

2013

Trata-se da versão corrigida da tese. A versão original se encontra disponível na EESC/USP, que aloja o Programa de Pós-Graduação de Engenharia Elétrica. 
AUTORIZO A REPRODUÇÃO TOTAL OU PARCIAL DESTE TRABALHO, DE ESTUDO E PESQUISA, DESDE QUE CITADA A FONTE.

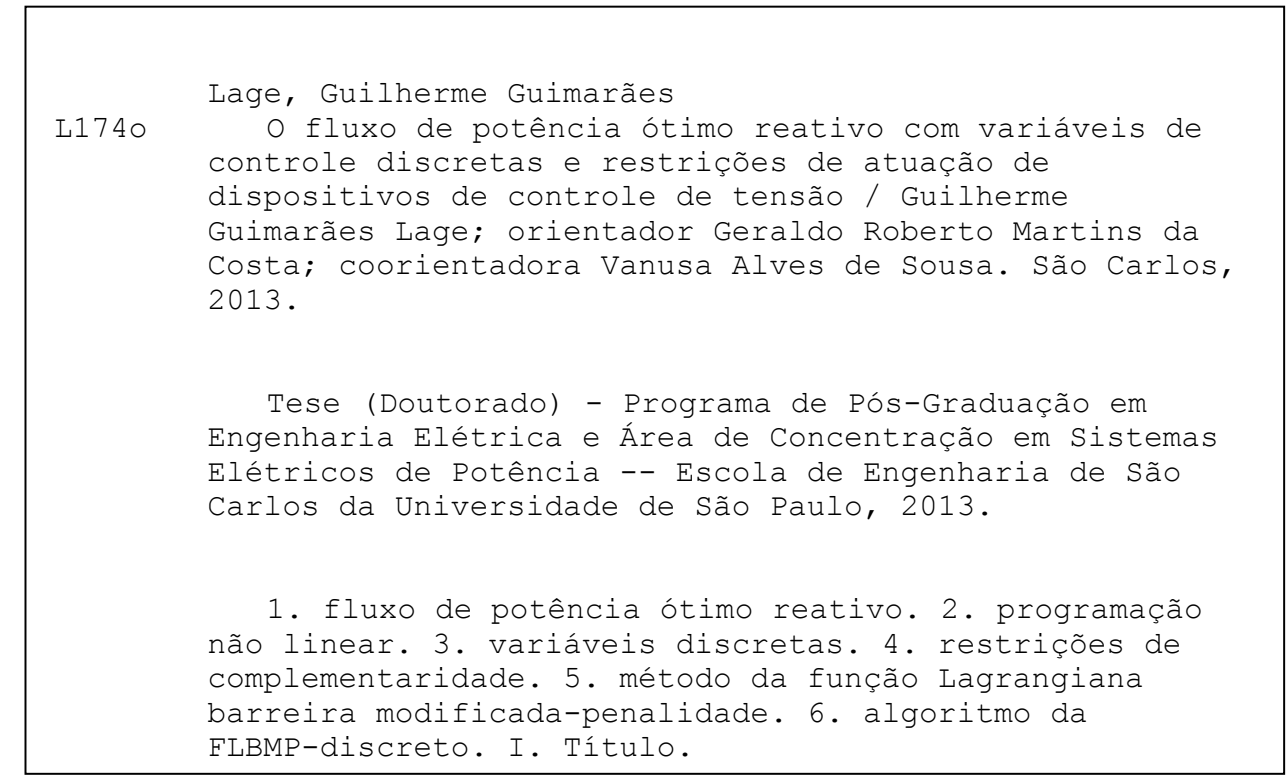




\section{FOLHA DE JULGAMENTO}

Candidato: Engenheiro GUILHERME GUIMARÃES LAGE.

Título da tese: "O fluxo de potência ótimo reativo com variáveis de controle discretas e restrições de atuação de dispositivos de controle de tensão".

Data da defesa: 25/03/2013

\section{Comissão Julgadora:}

Resultado:

Prof. Titular Geraldo Roberto Martins da Costa (Orientador) APROUNDO

(Escola de Engenharia de São Carlos/EESC)

Prof. Dr. José Carlos de Melo Vieira Júnior

APROVADO

(Escola de Engenharia de São Carlos/EESC)

Prof. Dr. Luiz Carlos Pereira da Silva

APROVADO

(Universidade Estadual de Campinas/UNICAMP)

Prof. Dr. Aurélio Ribeiro Leite de Oliveira

ARNOVADO

(Universidade Estadual de Campinas/UNICAMP)

Prof. Dr. Thales Sousa

(Universidade Federal do ABC)

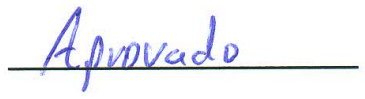

Coordenador do Programa de Pós-Graduação em Engenharia Elétrica e Presidente da Comissão de Pós-Graduação:

Prof. Titular Denis Vinicius Coury 

Aos meus pais, Eduardo e Vânia, e aos meus irmãos, Otávio e Vitor. 



\section{Agradecimentos}

Ao Professor Dr. Geraldo Roberto Martins da Costa, pela minha formação como pesquisador e pelos ensinamentos, orientação e amizade durante o desenvolvimento deste trabalho.

À Professora Dra. Vanusa Alves de Sousa, por toda ajuda e paciência dedicadas a mim no início do meu curso de doutorado.

Ao Professor Ph.D. Claudio Adrián Cañizares, pela receptividade e supervisão do meu trabalho durante o estágio de pesquisa em Waterloo, no Canadá.

À minha família e aos amigos de Patrocínio, que, mesmo distantes, sempre me deram força e me apoiaram ao longo desta longa jornada.

Aos amigos do Laboratório de Análise de Sistemas de Energia Elétrica (LASEE) e todos os outros que fiz ao longo desses anos em São Carlos, pelas nossas conversas, trocas de ideias, parcerias e momentos de descontração.

Aos professores e funcionários do Departamento de Engenharia Elétrica (SEL) da Escola de Engenharia de São Carlos (EESC), em especial à Jussara Ramos Zoia e à Marisa Helena R. Vicente Fortulan, pela colaboração, amizade e presteza.

À EESC, pela oportunidade de realização do curso de doutorado.

À Fundação de Amparo à Pesquisa do Estado de São Paulo (FAPESP) e à Coordenação de Aperfeiçoamento de Pessoal de Nível Superior (CAPES), pelas concessões de bolsas de doutorado direto e de estágio de pesquisa no exterior. 

O homem nasceu para aprender, aprender tanto quanto a vida lhe permita.

Guimarães Rosa 



\section{Resumo}

\section{LAGE, G. G. O fluxo de potência ótimo reativo com variáveis de controle}

discretas e restrições de atuação de dispositivos de controle de tensão. 2013. 234 f. Tese (Doutorado em Ciências, Programa de Engenharia Elétrica) - Escola de Engenharia de São Carlos, Universidade de São Paulo, São Carlos, 2013.

Este trabalho propõe um novo modelo e uma nova abordagem para resolução do problema de fluxo de potência ótimo reativo com variáveis de controle discretas e restrições de atuação de dispositivos de controle de tensão. Matematicamente, esse problema é formulado como um problema de programação não linear com variáveis contínuas e discretas e restrições de complementaridade, cuja abordagem para resolução proposta neste trabalho se baseia na resolução de uma sequência de problemas modificados pelo algoritmo da função Lagrangiana barreira modificada-penalidade-discreto. Nessa abordagem, o problema original é modificado da seguinte forma: 1) as variáveis discretas são tratadas como contínuas por funções senoidais incorporadas na função objetivo do problema original; 2) as restrições de complementaridade são transformadas em restrições de desigualdade equivalentes; e 3) as restrições de desigualdade são transformadas em restrições de igualdade a partir do acréscimo de variáveis de folga não negativas. Para resolver o problema modificado, a condição de não negatividade das variáveis de folga é tratada por uma função barreira modificada com extrapolação quadrática. O problema modificado é transformado em um problema Lagrangiano, cuja solução é determinada a partir da aplicação das condições necessárias de otimalidade. No 
algoritmo da função Lagrangiana barreira modificada-penalidade-discreto, uma sequência de problemas modificados é resolvida até que todas as variáveis do problema modificado associadas às variáveis discretas do problema original assumam valores discretos. Para demonstrar a eficácia do modelo proposto e a robustez dessa abordagem para resolução de problemas de fluxo de potência ótimo reativo, foram realizados testes com os sistemas elétricos IEEE de 14, 30, 57 e 118 barras e com o sistema equivalente CESP $440 \mathrm{kV}$ de 53 barras. Os resultados mostram que a abordagem para resolução de problemas de programação não linear proposta é eficaz no tratamento de variáveis discretas e restrições de complementaridade.

Palavras-chave: fluxo de potência ótimo reativo, programação não linear, variáveis discretas, restrições de complementaridade, método da função Lagrangiana barreira modificada-penalidade, algoritmo da FLBMP-discreto. 


\section{Abstract}

LAGE, G. G. The reactive optimal power flow with discrete control variables and voltage-control actuation constraints. 2013. 234 f. Tese (Doutorado em Ciências, Programa de Engenharia Elétrica) - Escola de Engenharia de São Carlos, Universidade de São Paulo, São Carlos, 2013.

This work proposes a novel model and a new approach for solving the reactive optimal power flow problem with discrete control variables and voltage-control actuation constraints. Mathematically, such problem is formulated as a nonlinear programming problem with continuous and discrete variables and complementarity constraints, whose proposed resolution approach is based on solving a sequence of modified problems by the discrete penalty-modified barrier Lagrangian function algorithm. In this approach, the original problem is modified in the following way: 1) the discrete variables are treated as continuous by sinusoidal functions incorporated into the objective function of the original problem; 2) the complementarity constraints are transformed into equivalent inequality constraints; and 3) the inequality constraints are transformed into equality constraints by the addition of non-negative slack variables. To solve the modified problem, the non-negativity condition of the slack variables is treated by a modified barrier function with quadratic extrapolation. The modified problem is transformed into a Lagrangian problem, whose solution is determined by the application of the first-order necessary optimality conditions. In the discrete penalty-modified barrier Lagrangian function algorithm, a sequence of modified problems is successively solved until all the variables of the modified 
problem that are associated with the discrete variables of the original problem assume discrete values. The effectiveness of the proposed model and the robustness of this approach for solving reactive optimal power flow problems were verified with the IEEE 14, 30, 57 and 118-bus test systems and the $440 \mathrm{kV}$ CESP 53-bus equivalent system. The results show that the proposed approach for solving nonlinear programming problems successfully handles discrete variables and complementarity constraints.

Keywords: reactive optimal power flow, nonlinear programming, discrete variables, complementarity constraints, penalty-modified barrier Lagrangian function method, discrete PMBLF algorithm. 


\section{Lista de Figuras}

Figura 4.1 - Convergência do método da FLBMP aplicado ao problema (4.27), para $x_{1}^{0}=1,1$ e $x_{2}^{0}=1,7 \ldots \ldots \ldots \ldots \ldots$

Figura 4.2 - Convergência do método da FLBMP aplicado ao problema (4.27), para $x_{1}^{0}=1,5$ e $x_{2}^{0}=1,0$ e multiplicadores $\sigma^{*}$ conhecidos. . . . . 88

Figura 5.1 - Representação gráfica do problema (5.6), onde $x \in \mathcal{X} . \ldots$. . . . 93

Figura 5.2 - Função $\psi$ definida por $x \in \mathcal{X}$, para $\beta=1 \ldots \ldots$. . . . . . . 94

Figura 5.3 - Função $\psi$ definida por $x \in \mathcal{X}$, para $\beta=2 \ldots \ldots \ldots$. . . . . 94

Figura 5.4 - Função $\psi$ definida por $x \in \mathcal{X}$, para $\beta=4 \ldots \ldots$. . . . . . 95

Figura 5.5 - Função objetivo aumentada definida por $x \in \mathcal{X}$, para $\gamma=0,3 \ldots$. . 96

Figura 5.6 - Função objetivo aumentada definida por $x \in \mathcal{X}$, para $\gamma=1,3 \ldots$. . 96

Figura 5.7 - Função objetivo aumentada definida por $x \in \mathcal{X}$, para $\gamma=3,0 \ldots \ldots 9$

Figura 5.8 - Representação gráfica do problema (5.6), onde $x \in \mathcal{X}^{\prime} \ldots$. . . . . 99

Figura 5.9 - Função objetivo aumentada definida por $x \in \mathcal{X}^{\prime}$, para $\gamma=0,3 . \quad$. . 99

Figura 5.10 - Função objetivo aumentada definida por $x \in \mathcal{X}^{\prime}$, para $\gamma=1,3 . \quad$. . 100

Figura 5.11 - Função objetivo aumentada definida por $x \in \mathcal{X}^{\prime}$, para $\gamma=3,0 . \quad$. . 100

Figura 5.12 - $1^{a}$ iteração do algoritmo da FLBMP-discreto aplicado ao problema (5.10) - Convergência do método da FLBMP aplicado ao problema modificado $(5.12) \ldots \ldots \ldots$. . . . . . . . . 105 
Figura 5.13 - $2^{a}$ iteração do algoritmo da FLBMP-discreto aplicado ao problema (5.10) - Convergência do método da FLBMP aplicado ao problema modificado $(5.12) \ldots \ldots \ldots$. . . . . . . . 105

Figura 5.14 - $3^{a}$ iteração do algoritmo da FLBMP-discreto aplicado ao problema (5.10) - Convergência do método da FLBMP aplicado ao problema modificado (5.12) . . . . . . . . . . . 106

Figura 5.15 - $4^{a}$ iteração do algoritmo da FLBMP-discreto aplicado ao problema (5.10) - Convergência do método da FLBMP aplicado ao problema modificado $(5.12) \ldots \ldots \ldots$. . . . . . . . 106

Figura $5.16-5^{a}$ iteração do algoritmo da FLBMP-discreto aplicado ao problema (5.10) - Convergência do método da FLBMP aplicado ao

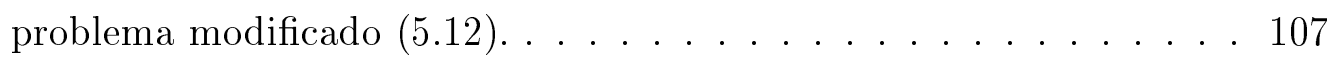

Figura 6.1 - Representação gráfica do problema de complementaridade (6.4). . . 111

Figura 6.2 - Representação gráfica do problema de complementaridade (6.6). . . 112

Figura 6.3 - Convergência do método da FLBMP aplicado ao problema (6.16). . . 122

Figura 8.1 - Modelo de um transformador em-fase. . . . . . . . . . . . . 132

Figura 8.2 - Circuito equivalente tipo $\pi$ de transformadores em-fase. . . . . . 133

Figura 8.3 - Modelo de um shunt de barra. . . . . . . . . . . . . 139

Figura 10.1 - Valor da susceptância equivalente do banco de capacitores do sistema IEEE de 14 barras ao longo do processo de resolução do FPOR (9.4). 153

Figura 10.2 - Valores dos taps dos transformadores do sistema IEEE 14 barras ao longo do processo de resolução do FPOR (9.4). . . . . . . . . . . . 154

Figura 10.3 - Perdas ativas no sistema IEEE de 14 barras ao longo do processo de resolução do FPOR (9.4). . . . . . . . . . . . . . . . . . 154

Figura 10.4 - Valor da susceptância equivalente do banco de capacitores do sistema IEEE de 14 barras ao longo do processo de resolução do FPOR (9.5). 157 
Figura 10.5 - Valores dos taps dos transformadores do sistema IEEE de 14 barras ao longo do processo de resolução do modelo (9.5) . . . . . . . . . 157

Figura 10.6 - Perdas ativas no sistema IEEE de 14 barras ao longo do processo de resolução do modelo $(9.5) \ldots$. . . . . . . . . . . . . 158

Figura 10.7 - Perfis das magnitudes de tensão determinados pelos modelos (9.3), (9.4) e (9.5) para o sistema IEEE de 14 barras. . . . . . . . . . . . 158

Figura 10.8 - Valores das susceptâncias equivalentes dos bancos de capacitores do sistema IEEE de 30 barras ao longo do processo de resolução do FPOR $(9.4) \ldots \ldots \ldots \ldots \ldots \ldots \ldots$

Figura 10.9 - Valores dos taps dos transformadores do sistema IEEE de 30 barras ao longo do processo de resolução do FPOR (9.4). . . . . . . . . . . 163

Figura 10.10 - Perdas ativas no sistema IEEE de 30 barras ao longo do processo de resolução do FPOR (9.4). . . . . . . . . . . . . . . . 164

Figura 10.11 - Valores das susceptâncias equivalentes dos bancos de capacitores do sistema IEEE de 30 barras ao longo do processo de resolução do FPOR $(9.5) \ldots \ldots \ldots \ldots \ldots \ldots$

Figura 10.12 - Valores dos taps dos transformadores do sistema IEEE de 30 barras ao longo do processo de resolução do modelo (9.5). . . . . . . . . 166

Figura 10.13 - Perdas ativas no sistema IEEE de 30 barras ao longo do processo de resolução do modelo $(9.5) \ldots \ldots$. . . . . . . . . . 167

Figura 10.14 - Perfis das magnitudes de tensão determinados pelos modelos (9.3), (9.4) e (9.5) para o sistema IEEE de 30 barras. . . . . . . . . . . 167

Figura 10.15 - Valores ótimos dos taps dos transformadores determinados pelo modelo (9.3) para o sistema IEEE de 57 barras. . . . . . . . . . 169

Figura 10.16 - Valores ótimos dos taps dos transformadores determinados pelo modelo (9.4) para o sistema IEEE de 57 barras. . . . . . . . . . . 170

Figura 10.17 - Valores ótimos dos taps dos transformadores determinados pelo modelo (9.5) para o sistema IEEE de 57 barras. . . . . . . . . . . 170 
Figura 10.18 - Valores ótimos das susceptâncias equivalentes dos bancos de capacitores e reatores shunt determinados pelo modelo (9.3) para o sistema IEEE de 118 barras. . . . . . . . . . . . . . 173

Figura 10.19 - Valores ótimos dos taps dos transformadores determinados pelo modelo (9.3) para o sistema IEEE de 118 barras. . . . . . . . . . 173

Figura 10.20 - Valores ótimos das susceptâncias equivalentes dos bancos de capacitores e reatores shunt determinados pelo modelo (9.4) para o sistema IEEE de 118 barras. . . . . . . . . . . . . . . . . 174

Figura 10.21 - Valores ótimos dos taps dos transformadores determinados pelo modelo (9.4) para o sistema IEEE de 118 barras. . . . . . . . . . . . 174

Figura 10.22 - Valores ótimos das susceptâncias equivalentes dos bancos de capacitores e reatores shunt determinados pelo modelo (9.5) para o sistema IEEE de 118 barras. . . . . . . . . . . . . . . . 175

Figura 10.23 - Valores ótimos dos taps dos transformadores determinados pelo modelo (9.5) para o sistema IEEE de 118 barras. . . . . . . . . . 175

Figura 10.24 - Valores ótimos dos taps dos transformadores determinados pelo modelo (9.3) para o sistema equivalente CESP $440 \mathrm{kV}$ de 53 barras.

Figura 10.25 - Valores ótimos dos taps dos transformadores determinados pelo modelo (9.4) para o sistema equivalente CESP $440 \mathrm{kV}$ de 53 barras.

Figura 10.26 - Valores ótimos dos taps dos transformadores determinados pelo modelo (9.5) para o sistema equivalente CESP $440 \mathrm{kV}$ de 53 barras. 178

Figura A.1 - Modelo equivalente $\pi$ de uma linha de transmissão. . . . . . . . . . 193

Figura A.2 - Modelo genérico de um transformador. . . . . . . . . . . . . . 195

Figura A.3 - Modelo de um shunt de barra. . . . . . . . . . . . . . . . . 197 


\section{Lista de Tabelas}

Tabela 4.1 - Convergência do método da FLBMP aplicado ao problema (4.27), para $x_{1}^{0}=1,1$ e $x_{2}^{0}=1,7$ - Função objetivo, variáveis primais e parâmetro $\mu \ldots \ldots \ldots \ldots \ldots \ldots$

Tabela 4.2 - Convergência do método da FLBMP aplicado ao problema (4.27), para $x_{1}^{0}=1,1$ e $x_{2}^{0}=1,7$ - Variáveis duais. . . . . . . . 85

Tabela 4.3 - Convergência do método da FLBMP aplicado ao problema (4.27), para $x_{1}^{0}=1,5$ e $x_{2}^{0}=1,0$ e multiplicadores $\sigma^{*}$ conhecidos - Função objetivo, variáveis primais e parâmetro $\mu \ldots \ldots$. . . . . 87

Tabela 4.4 - Convergência do método da FLBMP aplicado ao problema (4.27), para $x_{1}^{0}=1,5$ e $x_{2}^{0}=1,0$ e multiplicadores $\sigma^{*}$ conhecidos - Variáveis duais.

Tabela 5.1 - Convergência do algoritmo da FLBMP-discreto aplicado ao problema (5.10) - Função objetivo, variáveis primais e parâmetro $\mu$. . 103

Tabela 5.2 - Convergência do algoritmo da FLBMP-discreto aplicado ao problema (5.10) - Variáveis duais. . . . . . . . . . . . 104

Tabela 6.1 - Convergência do método da FLBMP aplicado ao problema (6.16) Função objetivo, variáveis $x$ e parâmetro $\mu$. . . . . . . . . 120

Tabela 6.2 - Convergência do método da FLBMP aplicado ao problema (6.16) Variáveis de folga $s$ e variáveis $c_{a}$ e $c_{b} \ldots \ldots \ldots 120$ 
Tabela 6.3 - Convergência do método da FLBMP aplicado ao problema (6.16) -

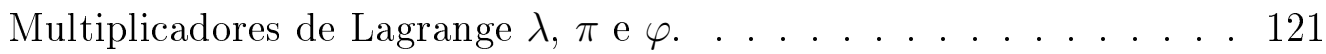

Tabela 6.4 - Convergência do método da FLBMP aplicado ao problema (6.16) Multiplicadores de Lagrange $\sigma . \ldots . . . . . . .121$

Tabela 10.1 - Limites mínimo e máximo da magnitude de tensão das barras controladas do sistema IEEE de 14 barras. . . . . . . . . . . . . . . . 150

Tabela 10.2 - Valores ótimos das variáveis de controle do sistema IEEE de 14 barras determinados pelo modelo (9.3). . . . . . . . . . . . 151

Tabela 10.3 - Valores ótimos das variáveis de controle do sistema IEEE de 14 barras

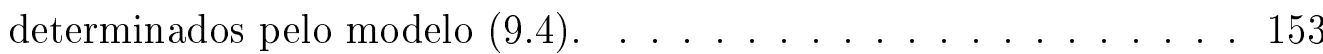

Tabela 10.4 - Valores ótimos das variáveis de controle do sistema IEEE de 14 barras determinados pelo modelo $(9.5) . \ldots \ldots \ldots . . \ldots 156$

Tabela 10.5 - Variáveis de controle em função dos limites mínimo e máximo da magnitude de tensão na barra 9. . . . . . . . . . . . . . . 159

Tabela 10.6 - Limites mínimo e máximo da magnitude de tensão das barras controladas do sistema IEEE de 30 de barras. . . . . . . . . . . . 160

Tabela 10.7 - Valores ótimos das variáveis de controle do sistema IEEE de 30 barras determinados pelo modelo $(9.3) . \ldots \ldots$. . . . . . . . 161

Tabela 10.8 - Valores ótimos das variáveis de controle do sistema IEEE de 30 barras determinados pelo modelo $(9.4) . \ldots \ldots$. . . . . . . 162

Tabela 10.9 - Valores ótimos das variáveis de controle do sistema IEEE de 30 barras determinados pelo modelo $(9.5) . \ldots \ldots . \ldots 165$

Tabela 10.10 - Valores ótimos das susceptâncias equivalentes dos shunts do sistema IEEE de 57 barras determinados pelos modelos (9.3), (9.4) e (9.5). 169

Tabela 10.11 - Valores das susceptâncias equivalentes dos reatores shunt e perdas no sistema equivalente CESP $440 \mathrm{kV}$ de 53 barras. . . . . . . . . . . . 177 


\section{Lista de Siglas}

$\begin{array}{ll}\text { AMPL } & \text { A Mathematical Programming Language } \\ \text { CESP } & \text { Companhia Energética de São Paulo } \\ \text { DE } & \text { Despacho Econômico } \\ \text { FBC } & \text { Função Barreira Clássica } \\ \text { FBM } & \text { Função Barreira Modificada } \\ \text { FBMEQ } & \text { Função Barreira Modificada com Extrapolação Quadrática } \\ \text { FC } & \text { Fluxo de Carga } \\ \text { FLBM } & \text { Função Lagrangiana Barreira Modificada } \\ \text { IPEPT } & \text { Interior Point Optimizer } \\ \text { FLBMP } & \text { Função Lagrangiana Barreira Modificada-Penalidade } \\ \text { FPO } & \text { Fluxo de Potência Ótimo } \\ & \text { Fluxo de Potência Ótimo Reativo de Minas e Energia } \\ & \end{array}$


MPI Método de Pontos Interiores

PB Programação Binível

PI Programação Inteira

PIM Programação Inteira-Mista

PL Programação Linear

PLIM Programação Linear Inteira-Mista

PNL Programação Não Linear

PQ Programação Quadrática

SEB Sistema Elétrico Brasileiro

SEE Sistema de Energia Elétrica 


\title{
Lista de Símbolos
}

\author{
$n_{p v} \quad$ número de barras do tipo $P V$ \\ $n_{p q} \quad$ número de barras do tipo $P Q$ \\ $\mathcal{B} \quad$ conjunto das barras do sistema \\ $\mathcal{G} \quad$ conjunto das barras de geração \\ $\mathcal{G}^{\prime} \quad$ conjunto das barras de geração, menos a barra slack \\ $\mathcal{C} \quad$ conjunto das barras de carga \\ $\mathcal{B}^{\text {sh }} \quad$ conjunto das barras com controle da magnitude de tensão por bancos de \\ capacitores
}

$\mathcal{L} \quad$ conjunto dos ramos $k-m$ que representam linhas de transmissão

$\mathcal{T} \quad$ conjunto dos ramos $k-m$ que representam transformadores em-fase com tap variável

$\mathcal{V}_{k} \quad$ conjunto das barras vizinhas à barra $k$

$\dot{E}_{k} \quad$ tensão fasorial na barra $k$

$V_{k} \quad$ magnitude da tensão na barra $k$

$\theta_{k} \quad$ ângulo de fase da tensão na barra $k$

$\theta_{k m} \quad$ abertura angular entre os fasores das tensões nas barras $k$ e $m$ 
$\dot{I}_{k m} \quad$ corrente fasorial que deixa a barra $k$ pelo ramo $k-m$

$\dot{I}_{k m}^{*} \quad$ conjugado complexo da corrente fasorial $\dot{I}_{k m}$

$\dot{I}_{k m}^{z} \quad$ componente série da corrente fasorial $\dot{I}_{k m}$

$\dot{I}_{k m}^{s h} \quad$ componente shunt da corrente fasorial $\dot{I}_{k m}$

$S_{k m} \quad$ fluxo potência complexa no ramo $k-m$

$P_{k m} \quad$ fluxo de potência ativa no ramo $k-m$

$Q_{k m} \quad$ fluxo de potência reativa no ramo $k-m$

$P_{k} \quad$ injeção líquida de potência ativa na barra $k$

$Q_{k} \quad$ injeção líquida de potência reativa na barra $k$

$Q_{k}^{s h} \quad$ injeção de potência reativa pelo shunt da barra $k$

$b_{k}^{s h} \quad$ susceptância equivalente do banco de capacitores da barra $k$

$\mathcal{D}_{k}^{s h} \quad$ conjunto dos valores discretos da susceptância equivalente do banco de capacitores da barra $k$

$b_{a_{k}} \quad$ variável auxiliar não negativa associada ao ajuste na susceptância do banco de capacitores ou reator shunt da barra $k$ quando a magnitude de tensão da barra controlada atinge seu limite mínimo

$b_{b_{k}} \quad$ variável auxiliar não negativa associada ao ajuste na susceptância do banco de capacitores ou reator shunt da barra $k$ quando a magnitude de tensão da barra controlada atinge seu limite máximo

$z_{k m} \quad$ impedância série do ramo $k-m$

$r_{k m} \quad$ resistência série do ramo $k-m$

$x_{k m} \quad$ indutância série do ramo $k-m$ 
$y_{k m} \quad$ admitância série do ramo $k-m$

$g_{k m} \quad$ condutância série do ramo $k-m$

$b_{k m} \quad$ susceptância série do ramo $k-m$

$b_{k m}^{s h} \quad$ susceptância shunt do modelo $\pi$ da linha de transmissão do ramo $k$ - $m$

$t_{k m} \quad$ tap do transformador em-fase do ramo $k-m$

$\mathcal{D}_{k m}^{\text {tap }} \quad$ conjunto dos valores discretos do tap do transformador em-fase do ramo $k$ - $m$

$t_{a_{k m}} \quad$ variável auxiliar não negativa associada ao ajuste no tap do transformador em-fase do ramo $k-m$ quando a magnitude de tensão da barra controlada atinge seu limite mínimo

$t_{b_{k m}} \quad$ variável auxiliar não negativa associada ao ajuste no tap do transformador em-fase do ramo $k-m$ quando a magnitude de tensão da barra controlada atinge seu limite máximo

$\varphi_{k m} \quad$ ângulo de defasamento do transformador defasador do ramo $k-m$

MW mega-watt

MVAr mega-volt-ampère reativo

$\mathbf{k V} \quad$ quilo-volt

p.u. por unidade 



\section{Sumário}

\section{Capítulo 1}

Introdução

1.1 Motivação . . . . . . . . . . . . . . . . . . 36

1.1.1 Modelagem das Variáveis de Controle Discretas . . . . . . . . . 37

1.1.2 Modelagem da Atuação de Dispositivos de Controle . . . . . . . . . 38

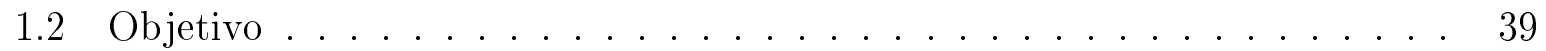

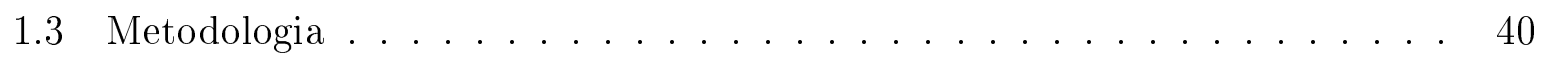

1.3.1 Forma de Análise dos Resultados . . . . . . . . . . . . . . . . 41

1.4 Organização do Trabalho . . . . . . . . . . . . . . . . . . . . . . 41

\section{Capítulo 2}

Histórico do Problema de FPO 43

2.1 Histórico do Problema de FPO . . . . . . . . . . . . . . 44

2.1.1 Variáveis de Controle Discretas . . . . . . . . . . . . . . 51

2.1.2 Restrições de Complementaridade . . . . . . . . . . . . . 55

\section{Capítulo 3}

$\begin{array}{ll}\text { Métodos de Otimização } & 57\end{array}$

3.1 Método Dual-Lagrangiano . . . . . . . . . . . . . 58

3.1 .1 Algoritmo . . . . . . . . . . . . . . . 60

3.1 .2 Dificuldades Computacionais . . . . . . . . . . . . . . . 61

3.2 Método de Newton-Lagrangiano . . . . . . . . . . . . . . 61 
3.2 .1 Algoritmo ...................... 63

3.2.2 Dificuldades Computacionais . . . . . . . . . . . . . . 63

3.3 Método de Penalidade . . . . . . . . . . . . . . . . . . 64

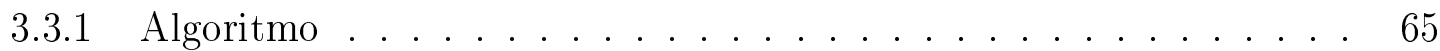

3.3.2 Dificuldades Computacionais . . . . . . . . . . . . . 66

3.4 Método de Barreira . . . . . . . . . . . . . . . 66

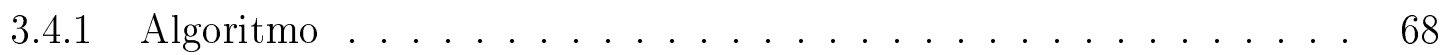

3.4.2 Dificuldades Computacionais . . . . . . . . . . . . . . 69

3.5 Método de Barreira Modificada . . . . . . . . . . . . . . 69

3.5.1 Algoritmo .......................... 71

3.5.2 Dificuldades Computacionais . . . . . . . . . . . . . 72

\section{Capítulo 4}

Método da Função Lagrangiana Barreira Modificada-Penalidade

4.1 Definição da FLBMP . . . . . . . . . . . . . . . . . . 75

4.2 Método da FLBMP . . . . . . . . . . . . . . . 78

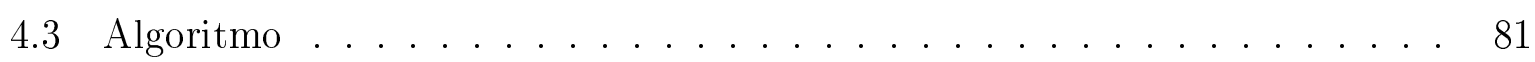

4.4 Exemplo Numérico . . . . . . . . . . . . . . . . . . 82

\section{Capítulo 5}

$\begin{array}{ll}\text { Algoritmo da FLBMP-Discreto } & 89\end{array}$

5.1 Definição do Problema Modificado . . . . . . . . . . . . 89

5.2 Algoritmo da FLBMP-Discreto . . . . . . . . . . . . . 91

5.2.1 Influência dos Parâmetros $\gamma$ e $\beta$ na Função $\psi \quad$. . . . . . . . . . . 92

$5.2 .2 \quad$ O Parâmetro $\alpha \ldots \ldots \ldots \ldots$. . . . . . . . . . . 97

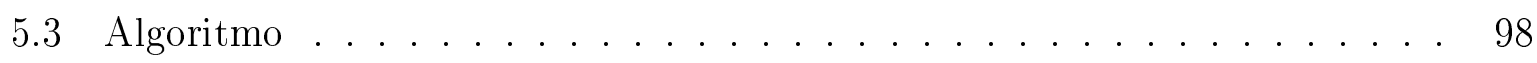

5.4 Exemplo Numérico . . . . . . . . . . . . . . . . . . . 101 


\section{Capítulo 6}

Problemas de PNL com Restrições de Complementaridade

6.1 Problemas de Complementaridade . . . . . . . . . . . . . . . 109

6.1.1 Condição de Complementaridade . . . . . . . . . . . . . . 109

6.1.2 Problemas de Complementaridade . . . . . . . . . . . . . . 110

6.2 Problemas de PNL com Restrições de Complementaridade . . . . . . . . . 114

6.3 Exemplo Numérico . . . . . . . . . . . . . . . . . . . 117

\section{Capítulo 7}

Modelagem Estática de Sistemas de Energia Elétrica

7.1 Modelagem Estática de Sistemas de Energia Elétrica . . . . . . . . . 123

7.2 Formulação do Problema de Fluxo de Carga . . . . . . . . . . . . . . . 125

7.2.1 Resolução do Problema de Fluxo de Carga . . . . . . . . . . . . . 128

\section{Capítulo 8}

Modelagem do Controle de Tensão por Problemas de Complementaridade

8.1 Modelagem da Atuação de Transformadores Em-fase . . . . . . . . . . . 132

8.2 Modelagem da Atuação de Bancos de Capacitores e Reatores Shunt . . . . 137

\section{Capítulo 9}

O Problema de FPOR

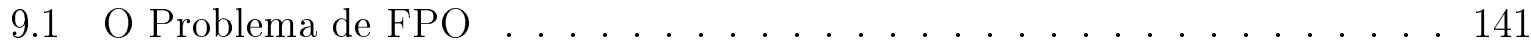

9.2 Formulação do Problema de FPOR . . . . . . . . . . . . . . . . . . 143

9.2.1 Modelo Clássico . . . . . . . . . . . . . . . . . . . . 143

9.2.2 Modelo com Variáveis de Controle Discretas . . . . . . . . . . . . . 145

9.3 FPOR com Variáveis de Controle Discretas e Restrições de Atuação de Dispositivos de Controle de Tensão . . . . . . . . . . . . . . . . . 146 


\section{Capítulo 10}

$\begin{array}{ll}\text { Resultados Numéricos } & 149\end{array}$

10.1 Sistema IEEE de 14 Barras . . . . . . . . . . . . . . . . . . 149

10.1.1 Modelo Clássico . . . . . . . . . . . . . . . . . . . . . 151

10.1.2 Modelo com Variáveis de Controle Discretas . . . . . . . . . . . . 152

10.1.3 Modelo com Variáveis de Controle Discretas e Restrições de Atuação de Dispositivos de Controle de Tensão . . . . . . . . . . . . 155

10.2 Sistema IEEE de 30 Barras . . . . . . . . . . . . . . . . . 159

10.2.1 Modelo Clássico . . . . . . . . . . . . . . . . . . . . 161

10.2.2 Modelo com Variáveis de Controle Discretas . . . . . . . . . . . . 161

10.2.3 Modelo com Variáveis de Controle Discretas e Restrições de Atuação de Dispositivos de Controle de Tensão . . . . . . . . . . . . . . . . 164

10.3 Sistema IEEE de 57 Barras $\ldots \ldots \ldots \ldots$. . . . . . . . . . 168

10.4 Sistema IEEE de 118 Barras . . . . . . . . . . . . . . . . . 171

10.5 Sistema Equivalente CESP $440 \mathrm{kV}$ de 53 Barras . . . . . . . . . . 176

\section{Capítulo 11}

Conclusões

11.1 Trabalhos Futuros . . . . . . . . . . . . . . . . 181

Referências Bibliográficas

Apêndice A

Expressões Gerais dos Fluxos de Potência Ativa e Reativa 191

A.1 Modelagem de Componentes do Sistema Elétrico . . . . . . . . . . . . . . 191

A.1.1 Linhas de Transmissão (MONTICELLI, 1983) . . . . . . . . . . 192

A.1.2 Transformadores Em-Fase e Defasadores (GROUP, 1973) . . . . . 194

A.1.3 Bancos de Capacitores e Reatores Shunt . . . . . . . . . . . 196

A.2 Expressões dos Fluxos de Potência . . . . . . . . . . . . 197 
A.2.1 Linhas de Transmissão . . . . . . . . . . . . . . . . . . . . 198

A.2.2 Transformadores Em-Fase e Defasadores . . . . . . . . . . . . 200

A.2.3 Expressões Gerais dos Fluxos . . . . . . . . . . . . . 203

A.3 Expressões das Injeções de Potência . . . . . . . . . . . . . . . . . . . . 204

\section{Apêndice B}

Relatórios dos Fluxos Ótimos $\quad 205$

B.1 Sistema IEEE 14 Barras . . . . . . . . . . . . . . . 205

B.2 Sistema IEEE 30 Barras . . . . . . . . . . . . . . . . . 208

B.3 Sistema IEEE 57 Barras . . . . . . . . . . . . . . . . . . 212

B.4 Sistema IEEE 118 Barras . . . . . . . . . . . . . . . . 220 



\section{Capítulo 1}

\section{Introdução}

A partir de 1995, o setor elétrico brasileiro passou por uma profunda reestruturação institucional e regulamentar, marcada pela mudança de um modelo de monopólio verticalmente integrado para um modelo de competição desverticalizada (ANEEL, 2008). Ao contrário do modelo antigo, totalmente regulado pelo Estado e verticalizado, no qual uma única empresa gerava, transmitia e distribuía energia elétrica para um grupo de consumidores cativos, no novo modelo, as empresas são divididas por atividade: geração, transmissão, distribuição, comercialização, exportação e importação; existe competição nos segmentos de geração e comercialização; e os segmentos de transmissão e distribuição, considerados monopólios naturais, são mantidos sob regulação do Estado (CCEE, 2012). Neste novo cenário, com as empresas do setor inseridas em um ambiente de mercado, o emprego de problemas de otimização no uso de recursos escassos e no auxílio à tomada de decisões desempenha um papel fundamental no planejamento e na operação dos Sistemas de Energia Elétrica (SEEs).

O planejamento e a operação dos SEEs devem considerar aspectos técnicos, financeiros, sociais e ambientais dentro de um contexto de regulação e leis que são diferentes para cada país. De forma geral, a tomada de decisões relacionadas ao planejamento e à operação dos SEEs é guiada por critérios de eficiência econômica para minimizar os custos 
de geração e transmissão da energia elétrica até os centros consumidores. No entanto, tais decisões são sujeitas, primeiramente, a restrições estritamente técnicas, que asseguram o fornecimento contínuo e de qualidade da energia elétrica demandada (GÓMEZ-EXPÓSITO; CONEJO; CAÑIZARES, 2011).

Entre os sistemas que integram um SEE, o sistema de transmissão, em especial, apresenta-se como uma grande fonte de complicações técnicas. Nesses sistemas, o efeito de fluxos em laço é um clássico exemplo que ilustra as complicações causadas pela existência de recursos críticos compartilhados, exibindo comportamentos característicos que são governados por leis físicas (leis de Kirchhoff), as quais não estão sujeitas aos contratos firmados pelas empresas do setor elétrico. Um outro exemplo que ilustra essa fonte de complicações técnicas é a determinação dos ajustes necessários para levar o sistema de volta ao estado de operação normal quando limites operacionais ou de segurança são violados. Em situações localizadas, o simples ajuste em um dispositivo de controle por um operador experiente pode resolver o problema. Porém, quando um grande número de limites forem violados, metodologias baseadas na resolução de problemas de Fluxo de Potência Ótimo (FPO) são a melhor alternativa, retirando dos operadores do sistema a responsabilidade da determinação dos melhores ajustes necessários (RAMOS et al., 1995).

Problemas de FPO constituem uma ampla classe de problemas cujo objetivo é a otimização de um dado desempenho operacional do SEE, sujeito ao balanço de potência ativa e reativa nas barras da rede, às restrições operacionais do sistema, aos limites das variáveis de controle etc. Diferentemente do problema de Fluxo de Carga (FC), nos problemas de FPO, as variáveis de controle do SEE são passíveis de ajustes para que um desempenho operacional ótimo do sistema seja determinado. Matematicamente, o FPO é formulado como um problema de Programação Não Linear (PNL), estático, não convexo, restrito, de grande porte e com variáveis contínuas e discretas. Desde sua proposição inicial por Carpentier, em 1962, o FPO tem se mostrado como uma ferramenta essencial para o planejamento e a operação dos SEEs. No planejamento, o FPO é empregado na determinação de cenários ótimos na evolução futura do sistema elétrico. 
Na operação, o FPO permite a determinação de ações de controle ótimas respeitando-se as restrições técnico-operacionais desses sistemas. Por esses motivos, o FPO aparece nos dias atuais como uma poderosa ferramenta para análise de SEEs graças ao desenvolvimento de metodologias eficientes (HUNEAULT; GALIANA, 1991; MOMOH; EL-HAWARY; ADAPA, 1999a; MOMOH; EL-HAWARY; ADAPA, 1999b) e à implementação de solvers robustos para a resolução desses problemas (QUINTANA; TORRES; MEDINA-PALOMO, 2000).

Apesar de todas as conquistas alcançadas com a reestruturação do setor elétrico, as margens de segurança operacional dos SEEs têm sido reduzidas, em parte, devido ao planejamento e à operação pautados por critérios de eficiência econômica. Em resposta às pressões de mercado, a operação dos SEEs, mais especificamente dos sistemas de transmissão, tem sido feita cada vez mais próxima aos seus limites de controle (ROSEHART, 2002; AFFONSO; SILVA; SILVA, 2008). Esta é uma característica inerente ao novo modelo do setor elétrico, no qual a tomada de decisões exige a escolha de um objetivo em detrimento de outro (MANKIW, 2009). Devido ao aumento da demanda por energia elétrica e à capacidade limitada do sistema de transmissão de acomodar cargas adicionais mantendo, ao mesmo tempo, um perfil de tensão adequado a diferentes cenários de operação, problemas de FPO aplicados a estudos de potência reativa têm despertado o interesse das empresas do setor elétrico e dos pesquisadores da área (SOUSA, 2006).

Tendo em vista a necessidade de operar SEEs de acordo com critérios de eficiência econômica e a crescente complexidade operacional dos sistemas de transmissão, este trabalho propõe um novo modelo e uma nova abordagem para resolução do problema de minimização das perdas ativas na transmissão, formulado como um problema de Fluxo de Potência Ótimo Reativo (FPOR). Nesses problemas de FPO, as variáveis de controle associadas à potência ativa são fixas e as variáveis de controle associadas à potência reativa são ajustadas de forma a otimizar o desempenho desejado.

A motivação para o desenvolvimento deste trabalho e os principais desafios associados à formulação e resolução de problemas de FPOR são discutidos a seguir. 


\subsection{Motivação}

Um dos objetivos de um FPO é a determinação dos ajustes das variáveis de controle de um SEE responsáveis por otimizar um determinado desempenho operacional do sistema. Através da resolução do problema minimização das perdas ativas na transmissão, determina-se, portanto, o conjunto de ações de controle que levam o sistema à uma operação mais eficiente e com menor custo operacional.

A motivação para o desenvolvimento de uma metodologia que dá suporte à tomada de decisões operacionais baseada na resolução desse problema de FPOR relaciona-se ao seu potencial de contribuir para um melhor desempenho do sistema de transmissão de energia elétrica, com um melhor perfil de tensão. Em estudos do sistema Sul-Sudeste brasileiro, as perdas ativas foram reduzidas em 3,53\%, mantendo-se um perfil de tensão médio em torno de 1,010 p.u. (BAPTISTA et al., 2006). De acordo com o Ministério de Minas e Energia (MME) do Brasil, a demanda média do Sistema Elétrico Brasileiro (SEB) entre outubro de 2011 e setembro de 2012 foi de aproximadamente 61.176,80 MW (BRASIL, 2012a). Perdas ativas na transmissão da ordem de $8,5 \%^{1}$ corresponderam, portanto, a aproximadamente 5.200 MW da potência média demandada nesse período. Nesse cenário, uma redução de 3,5\% nas perdas corresponderia a uma economia de aproximadamente $182 \mathrm{MW}$ médios. Isto não pode ser ignorado, pois essa economia pode ser obtida atuando-se exclusivamente sobre as variáveis de controle do sistema, sem a necessidade de investimentos adicionais em infraestrutura.

Dada a importância desse problema ao planejamento e à operação dos SEEs, passa-se agora à discussão de dois fatores que podem, dependendo da abordagem para resolução empregada, inviabilizar formulações mais realistas de problemas de FPO: a modelagem das variáveis de controle discretas e a modelagem da atuação de dispositivos de controle de tensão.

\footnotetext{
${ }^{1}$ Perdas ativas na transmissão que correspondem a pouco menos da metade das perdas totais nos SEEs são bem aceitas. De outubro de 2011 a setembro de 2012, as perdas totais registradas no SEB variaram entre $15,0 \%$ e 20,3\% da potência média mensal demandada (BRASIL, 2011; BRASIL, 2012b).
} 


\subsubsection{Modelagem das Variáveis de Controle Discretas}

Em muitos problemas de análise estática de SEEs, as variáveis de controle associadas aos taps de transformadores em-fase e chaveamentos de bancos de capacitores e indutores shunt são consideradas contínuas e, posteriormente, ajustadas, ou "arredondadas", para seu valor discreto mais próximo. No entanto, em problemas de FPO, essa abordagem pode descaracterizar a solução ótima do problema (LIU; PAPALEXOPOULOS; TINNEY, 1992) ou até resultar em soluções infactíveis (SOLER, 2011).

Problemas de PNL com variáveis contínuas e discretas são geralmente resolvidos por métodos de Programação Inteira-Mista (PIM). No entanto, a complexidade computacional da abordagem combinatorial para determinação de uma solução ótima desses problemas tende a aumentar exponencialmente com o número de variáveis discretas. Métodos como Outer Approximation, Decomposição de Benders, Branch-and-Bound e algoritmos de Planos de Corte são não polinomiais e requerem um grande esforço computacional quando aplicados a problemas de grande porte que envolvem um grande número de variáveis discretas (SOLER, 2011). Métodos meta-heurísticos incluem heurísticas de construção e busca local que exploram o espaço de soluções, porém podem demandar um alto tempo computacional (ÖSTERMARK, 2004). Neste contexto, a modelagem estritamente discreta de variáveis de controle e o uso de métodos de PIM inviabilizam formulações mais realistas de problemas de FPOR para análise de SEEs de grande porte.

Devido às dificuldades impostas pela existência de variáveis de controle discretas nos modelos de SEEs, a maioria das abordagens para resolução de problemas de FPO propostas na literatura emprega métodos clássicos de PNL, desconsiderando a modelagem discreta dessas variáveis. Essas abordagens, no entanto, estão longe da realidade de um SEE, pois alguns de seus controles só podem ser ajustados por passos discretos.

Dessa forma, opta-se neste trabalho pelo emprego da abordagem proposta por Soler, Sousa e Costa (2012) para o tratamento das variáveis de controle discretas em 
problemas de FPOR. Nela, as variáveis discretas do problema de FPOR são tratadas como contínuas por funções senoidais $\psi$ incorporadas na função objetivo do problema original. Essas funções se anulam quando essas variáveis assumirem valores discretos e sua amplitude é controlada por um parâmetro $\gamma>0$.

\subsubsection{Modelagem da Atuação de Dispositivos de Controle}

Os operadores de SEEs procuram ajustar o menor número de controles em um intervalo de tempo para o alcance de um determinado desempenho operacional do sistema. No entanto, os modelos e algoritmos para resolução de problemas de FPO existentes "utilizam" todas as variáveis de controle na determinação do desempenho operacional ótimo. Nesse caso, um número excessivo de ações de controle torna a solução obtida impraticável à operação de sistemas de grande porte.

Portanto, torna-se necessário restringir, sempre que possível, os ajustes em algumas variáveis de controle no processo de determinação da operação ótima praticável de um SEE. Esse requisito é especialmente importante para metodologias que dão suporte à tomada de decisões operacionais pela resolução de problemas de FPOR, pois um número muito grande de ajustes nas variáveis de controle (principalmente nas variáveis associadas a controles ajustados por passos discretos) é praticamente impossível de ser realizado antes que o estado do sistema mude significativamente.

Matematicamente, o controle de tensão por ajustes nos taps de transformadores em-fase e chaveamentos de bancos de capacitores e reatores shunt pode ser representado por problemas de complementaridade, os quais modelam algebricamente a condição de que esses dispositivos atuem somente quando um dos limites de tensão da barra controlada por eles for atingido. Dessa forma, através da incorporação desses problemas de complementaridade no conjunto de restrições de problemas de FPOR, restringem-se, portanto, os ajustes nas variáveis de controle associadas a dispositivos de controle de tensão. 
Todavia, métodos de otimização que empregam o cálculo de matrizes Hessianas no processo de resolução de problemas de PNL com restrições de complementaridade apresentam problemas numéricos em pontos de complementaridade não estrita ${ }^{2}$, pois a matriz Hessiana da função Lagrangiana se torna singular nesses pontos (ROSEHART; ROMAN; SCHELLENBERG, 2005).

Por esse motivo, restrições de complementaridade são, de acordo com as propostas na literatura, reformuladas pela função Fischer-Burmeister (PETOUSSIS; ZHANG; GODFREY, 2007; TODOROV, 2010; JIN; RYAN, 2011; ALMEIDA; SENNA, 2011), transformadas em restrições de desigualdade equivalentes (BENSON; SHANNO; VANDERBEI, 2002; RAGHUNATHAN; BIEGLER, 2005), ou tratadas por uma função penalidade (LEYFFER; LÓPEZ-CALVA; NOCEDAL, 2006). Porém, nas reformulações pela função Fischer-Burmeister, métodos clássicos de PNL podem continuar apresentando problemas numéricos, pois as derivadas de primeira e segunda ordem dessa função tendem ao infinito em pontos de complementaridade não estrita. A abordagem pela função penalidade pode trazer problemas de mal condicionamento à matriz Hessiana à medida que o valor do parâmetro de penalidade cresça.

Dessa forma, opta-se neste trabalho por transformar as restrições de complementaridade em restrições de desigualdade equivalentes (BENSON; SHANNO; VANDERBEI, 2002).

\subsection{Objetivo}

As proposições de novos modelos de problemas de FPO acompanham de perto o desenvolvimento de técnicas robustas e eficientes para sua resolução. Historicamente, problemas de FPO são resolvidos por métodos clássicos de PNL, ou uma associação destes, considerando as variáveis de controle discretas como contínuas e utilizando todos controles na determinação do desempenho operacional ótimo do sistema. Essas abordagens, no entanto, estão muito longe da realidade de um SEE, pois alguns de seus controles só

\footnotetext{
${ }^{2}$ A definição de pontos de complementaridade não estrita é apresentada no Capítulo 6 .
} 
podem ser ajustados por passos discretos, e a atuação irrestrita de dispositivos de controle é impraticável à operação de sistemas de grande porte.

Nesse contexto, este trabalho propõe um novo modelo e uma abordagem para resolução do problema de minimização das perdas ativas na transmissão, com variáveis de controle discretas e restrições de atuação de dispositivos de controle de tensão.

\subsection{Metodologia}

Matematicamente, o FPOR será formulado como um problema de PNL com variáveis contínuas e discretas e restrições de complementaridade, cuja abordagem para resolução se baseia na resolução de uma sequência de problemas pelo algoritmo da Função Lagrangiana Barreira Modificada-Penalidade (FLBMP)-discreto.

Nessa abordagem, o problema de FPOR é modificado da seguinte forma:

1) as variáveis discretas são tratadas como contínuas por funções senoidais $\psi$;

2) as restrições de complementaridade são transformadas em restrições de desigualdade equivalentes;

3) e as restrições de desigualdade são transformadas em restrições de igualdade a partir do acréscimo de variáveis de folga não negativas.

Para resolver o problema modificado, a condição de não negatividade das variáveis de folga é tratada por uma Função Barreira Modificada com Extrapolação Quadrática (FBMEQ) (BREITFELD; SHANNO, 1994; BREITFELd; SHANNO, 1996). O problema modificado é transformado em um problema Lagrangiano, cuja solução é determinada a partir da aplicação das condições necessárias de otimalidade. Esta abordagem para resolução de problemas de PNL é denominada método da FLBMP.

No algoritmo da FLBMP-discreto, uma sequência de problemas é resolvida pelo método da FLBMP até que todas as variáveis do problema modificado associadas às variáveis discretas do problema original assumam valores discretos. 


\subsubsection{Forma de Análise dos Resultados}

Para verificar a eficácia do modelo proposto e a robustez da abordagem para resolução de problemas de FPOR desenvolvida, serão realizados testes com os sistemas elétricos IEEE de 14, 30, 57 e 118 barras e com o sistema equivalente CESP $440 \mathrm{kV}$ de 53 barras.

\subsection{Organização do Trabalho}

Este trabalho está organizado da seguinte forma.

No Capítulo 2 é apresentado o histórico das principais abordagens para resolução de problemas de FPO desde sua proposição inicial por Carpentier em 1962, incluindo propostas de abordagens para resolução desses problemas com variáveis de controle discretas e restrições de complementaridade.

No Capítulo 3 apresenta-se uma revisão dos métodos de otimização que dão suporte teórico para o desenvolvimento da abordagem para resolução de problemas de PNL proposta neste trabalho. São apresentados os métodos dual-Lagrangiano, de Newton-Lagrangiano, de penalidade, de barreira e de barreira modificada

No Capítulo 4 é apresentado o método da FLBMP para resolução de problemas de PNL restritos.

No Capítulo 5 apresenta-se o algoritmo da FLBMP-discreto para resolução de problemas de PNL com variáveis contínuas e discretas.

No Capítulo 6 o método da FLBMP é aplicado à resolução de problemas de PNL com restrições de complementaridade.

No Capítulo 7 são apresentadas a modelagem estática de um SEE e a formulação básica do problema de FC, a partir do qual determina-se o estado da rede em função dos ajustes dos controles do sistema elétrico. 
No Capítulo 8 os controles da magnitude de tensão por ajustes nos taps de transformadores em-fase e chaveamentos de bancos de capacitores e reatores shunt são modelados como problemas de complementaridade.

No Capítulo 9 são apresentados a formulação de problemas de FPOR e o modelo proposto neste trabalho para o problema de minimização das perdas ativas na transmissão com variáveis de controle discretas e restrições de atuação de dispositivos de tensão.

No Capítulo 10 são apresentados os testes realizados com o modelo de FPOR e com a abordagem de resolução propostos neste trabalho.

E, finalmente, no Capítulo 11 são apresentadas as considerações finais a respeito do trabalho aqui apresentado, bem como as perspectivas de continuação deste. 


\section{Capítulo 2}

\section{Histórico do Problema de FPO}

Em 1962, Carpentier propôs uma nova formulação do problema de Despacho Econômico (DE) pela incorporação das equações do problema de FC no conjunto das restrições desse problema. Dessa forma, além de determinar o despacho ótimo de potência ativa ao menor custo operacional, respeitando-se os limites de cada unidade de geração, a formulação proposta por Carpentier calcula o FC na rede e, consequentemente, seu estado ótimo. Para resolver esse problema, Carpentier transformou-o em um problema irrestrito por uma função Lagrangiana clássica e resolveu o sistema de equações não lineares resultante da aplicação das condições necessárias de primeira ordem ao problema Lagrangiano pelo método de Gauss-Seidel. A solução do problema de DE é determinada quando as condições de Karush-Kuhn-Tucker (KKT) forem satisfeitas.

Após essa formulação do problema de DE, várias outras formulações de problemas de otimização aplicados à análise estática de SEEs e/ou propostas de abordagens para resolução destes foram publicadas. Desde então, qualquer problema que envolva a determinação do estado ótimo de um SEE em função de um determinado desempenho operacional é chamado de problema de FPO. O problema DE passou, portanto, a ser considerado um caso particular de problema de FPO. Neste capítulo, é apresentado o histórico das principais abordagens para resolução de problemas de FPO, incluindo 
propostas de abordagens para resolução desses problemas com variáveis de controle discretas e restrições de complementaridade.

\subsection{Histórico do Problema de FPO}

Dommel e Tinney (1968) propuseram a resolução de problemas de FPO pelo método do gradiente reduzido, que, por sua vez, consiste em uma abordagem de primeira ordem para determinação dos ajustes ótimos das variáveis de controle de um SEE por passos descendentes na função objetivo. Nessa abordagem, o problema de FPO é transformado em um problema irrestrito por uma função Lagrangiana. As restrições de desigualdade e os limites das variáveis dependentes são incorporados à função objetivo por penalidades quadráticas. Dado um conjunto de ajustes das variáveis de controle do SEE, a resolução do sistema de equações não lineares resultante da aplicação das condições necessárias de primeira ordem ao problema Lagrangiano é feita em três partes: 1) o estado da rede é determinado através da resolução do problema de FC pelo método de Newton (TINNEY; HART, 1967); 2) calculam-se os multiplicadores de Lagrange associados às restrições de igualdade do problema; e 3) os ajustes das variáveis de controle do SEE são determinados pelo gradiente negativo da função objetivo em relação a essas variáveis. O tamanho do passo para o ajuste das variáveis de controle do sistema é aquele que proporciona o maior decréscimo no valor da função objetivo. Na atualização das variáveis de controle, as variáveis cujos limites são violados são tratadas pelo método da projeção. O método continua, iterativamente, até que a norma do gradiente da função Lagrangiana em relação às variáveis de controle do SEE seja menor que uma tolerância predeterminada. Apesar do seu rigor matemático, o cálculo do tamanho do passo para o ajuste nas variáveis de controle é um fator crítico para o bom desempenho desta abordagem: passos muito pequenos garantem uma convergência local mas demandam muitas iterações do algoritmo; passos muito grandes fazem com que o algoritmo fique "ziguezagueando" nas proximidades da solução ótima. 
Sasson (1969) apresentou uma abordagem para a resolução de problemas de FPO baseada na associação dos métodos de Powell, para problemas de PNL restritos, e de Fletcher-Powell, para problemas de PNL irrestritos. Nela, o problema de FPO é transformado em um problema de minimização irrestrito pela incorporação das restrições de igualdade e desigualdade violadas à função objetivo do problema original por dois parâmetros distintos que controlam o processo de convergência do método. Em seguida, aplica-se o método de Fletcher-Powell, que de fato minimiza a função de Powell. Nessa abordagem, todas as variáveis do SEE são ajustadas simultaneamente por um vetor direção de busca determinado pela multiplicação entre uma aproximação da matriz Hessiana inversa da função de Powell e o gradiente da função de Powell. Essa matriz é construída iterativamente pelo método de Fletcher-Powell e corresponde à matriz Hessiana inversa da função de Powell na solução do problema. Embora o método de Fletcher-Powell possua uma característica de convergência quadrática, essa matriz Hessiana inversa é cheia. Além disso, esta abordagem apresenta problemas de convergência quando aplicada a SEEs de grande porte.

Sasson, Viloria e Aboytes (1973) propuseram o uso do método de penalidade em uma abordagem de segunda ordem para resolução dos problemas de FPO. Nessa abordagem, o problema de FPO é transformado em um problema irrestrito da seguinte forma: as restrições de igualdade e as restrições de desigualdade violadas são incorporadas à função objetivo por penalidades quadráticas, definindo, assim, uma função auxiliar. Essa função auxiliar é, então, minimizada pelo método de Newton. A cada iteração do método de penalidade, a matriz Hessiana dessa função auxiliar é calculada, todas as variáveis são atualizadas simultaneamente e, caso não haja a convergência do processo para uma solução factível, os fatores de penalidade são aumentados. A convergência desse processo se dá quando todas as restrições de igualdade e de desigualdade forem satisfeitas. A matriz Hessiana da função auxiliar é esparsa, possibilitando o uso de técnicas de esparsidade no processo computacional, mas, como é diretamente influenciada pelo parâmetro de penalidade, ela pode se tornar mal condicionada à medida que esse parâmetro cresça. 
No intuito de superar algumas deficiências da abordagem proposta por Dommel e Tinney (1968), Rashed e Kelly (1974) propuseram uma abordagem de segunda ordem para resolução de problemas de FPO pelo método do gradiente reduzido. Nessa abordagem, o problema de FPO é transformado em um problema irrestrito pelo método dos multiplicadores de Lagrange. As restrições de desigualdade e os limites das variáveis dependentes são incorporados à função objetivo por penalidades quadráticas. Dado um conjunto de ajustes das variáveis de controle do SEE, a resolução das condições necessárias de primeira ordem é feita em três partes: 1) o estado da rede é determinado através da resolução do problema de FC; 2) calculam-se os multiplicadores de Lagrange associados às restrições de igualdade do problema; e 3) os ajustes das variáveis de controle do SEE são determinados pela matriz Hessiana da função Lagrangiana definida em relação a essas variáveis. Essa matriz Hessiana é altamente esparsa e de menor dimensão que a matriz Hessiana da função auxiliar do método proposto por Sasson, Viloria e Aboytes (1973). Na atualização das variáveis de controle, as variáveis cujos limites são violados são tratadas pelo método da projeção. O método continua, iterativamente, até que uma condição de convergência, como a variação na função objetivo ou o módulo do maior ajuste nas variáveis de controle, seja menor que uma tolerância predeterminada. O algoritmo proposto foi aplicado, no entanto, a um sistema de 5 barras.

Sun et al. (1984) propuseram a resolução de problemas de FPO pelo método de Newton. Nessa abordagem, o problema de FPO é transformado em um problema irrestrito da seguinte forma: as restrições de igualdade e as restrições de desigualdade ativas na solução são incorporadas à função objetivo do problema por multiplicadores de Lagrange, e os limites das variáveis de controle são incorporados à função objetivo por penalidades quadráticas. A resolução das condições necessárias de primeira ordem pelo método de Newton é desacoplada em dois subproblemas: um subproblema de potência ativa e um subproblema de potência reativa. De acordo com os autores, esta abordagem é eficiente e robusta para resolução de problemas de FPO, independente do tamanho do SEE e do número de controles e restrições ativas na solução, e o esforço computacional é proporcional 
ao tamanho do sistema. Cada iteração minimiza uma aproximação quadrática da função Lagrangiana, e a ideia chave desta abordagem é o ajuste simultâneo nas variáveis primais e duais da função Lagrangiana a cada iteração. Para um dado conjunto de restrições ativas, o processo satisfaz as condições de KKT em poucas iterações. No entanto, esta abordagem tem como desafio, no desenvolvimento do seu algoritmo, a identificação das restrições de desigualdade ativas na solução.

Santos Jr., Deckmann e Soares (1988) aplicaram o método da função Lagrangiana aumentada à resolução de problemas FPO. Nele, o problema de FPO é transformado em um problema irrestrito por uma função Lagrangiana clássica, na qual as restrições de igualdade e desigualdade são incorporadas à função objetivo por multiplicadores de Lagrange. Em seguida, essa função Lagrangiana é aumentada pela incorporação de todas as restrições de igualdade e desigualdade do problema original por penalidades quadráticas. De acordo com esta abordagem, a função Lagrangiana aumentada é minimizada em relação às variáveis primais pelo método de Newton, os multiplicadores de Lagrange são atualizados no sentido de maximizar a função Lagrangiana aumentada, e a atualização do parâmetro de penalidade é feita de forma a garantir a existência do ponto de sela dessa função Lagrangiana. A trajetória de convergência do método ocorre pela região infactível do problema. O método da função Lagrangiana aumentada pode ser considerado um aperfeiçoamento dos métodos propostos por Sasson, Viloria e Aboytes (1973) e Sun et al. (1984), pois, nele, o mal condicionamento da matriz Hessiana da função Lagrangiana aumentada é evitado, já que o método controla a atualização do parâmetro de penalidade, e não há a necessidade de identificar as restrições ativas na solução.

Huneault e Galiana (1991) apresentaram um estudo sobre abordagens de resolução de problemas de FPO através de uma revisão bibliográfica de mais de trezentas publicações na área. Nesse trabalho, os autores evidenciam a evolução de modelos com o passar dos anos e classificam os métodos existentes de acordo com as técnicas de programação que eles empregam. Os autores ainda afirmam que apesar dos avanços obtidos na área, problemas de FPO ainda são sujeitos a situações de mal condicionamento e de difícil convergência. 
Granville (1994) propôs a resolução do problema de FPOR pelo método primal-dual barreira logarítmica. Esta foi uma das primeiras propostas de resolução de problemas de FPO por um Método de Pontos Interiores (MPI). Nessa abordagem, o problema de FPOR é transformado em um problema irrestrito da seguinte forma: 1) as restrições de desigualdade são transformadas em restrições de igualdade a partir do acréscimo de variáveis de folga não negativas; 2) a condição de não negatividade das variáveis de folga é tratada por uma função barreira logarítmica, que, por sua vez, é incorporada à função objetivo por um parâmetro de barreira; e 3) as restrições de igualdade, originais e transformadas, são incorporadas à função objetivo por multiplicadores de Lagrange. O problema de barreira é resolvido pelo método de Newton, monitorando-se o ajuste das variáveis de folga e duais associadas às restrições de igualdade transformadas a cada iteração. Após a convergência do método de Newton, o parâmetro de barreira é atualizado por uma regra de redução, e o novo problema de barreira é resolvido. A solução do problema de FPOR é encontrada quando todas as restrições forem satisfeitas e o parâmetro de barreira tender a zero. Esta abordagem exige que a estimativa inicial da solução do problema seja factível e apresenta muita sensibilidade quanto à escolha do parâmetro de barreira inicial, podendo até divergir em alguns casos.

No mesmo ano, Wu, Debs e Marsten (1994) também propuseram o uso do método primal-dual barreira logarítmica para resolução de problemas de FPO, porém utilizaram uma variante do método de Newton-Raphson denominada preditor-corretor. Essa modificação torna a sua convergência mais rápida que a da abordagem proposta por Granville (1994).

Torres e Quintana (1998) propuseram a resolução de problemas de FPO na forma retangular, e não na polar, por um método de pontos interiores. Dessa forma, problemas de FPO podem ser formulados por funções quadráticas, o que torna a matriz Hessiana da função Lagrangiana constante. Os autores utilizaram a abordagem para resolução proposta por Granville (1994) e apresentaram técnicas para a escolha do tamanho do passo na determinação das direções de busca, para a redução do fator de barreira e para o 
uso de um método preditor-corretor. Os autores afirmam, no entanto, que o desempenho computacional de métodos de pontos interiores é praticamente igual para problemas de FPO formulados tanto na forma polar quanto na forma retangular.

Momoh, El-Hawary e Adapa (1999a), Momoh, El-Hawary e Adapa (1999b) apresentaram uma ampla revisão bibliográfica sobre abordagens para resolução de problemas de FPO, dividindo-as em seis categorias: PNL, onde citam o uso técnicas de minimização irrestrita sequencial e métodos Lagrangianos; Programação Quadrática (PQ), referindo-se aos métodos quasi-Newton e de sensibilidade; solução das condições de KKT baseadas no método de Newton; Programação Linear (PL), onde comentam sobre a utilização do método Simplex e do método Simplex revisado; PIM, que consistem na associação de técnicas de PL e Programação Inteira (PI); e, finalmente, MPIs.

Costa, Costa e Souza (2000) apresentaram um estudo comparativo de três abordagens para resolução de problemas de FPO, todas elas baseadas no método de Newton. Essas abordagens são: penalidade e conjunto ativo, primal-dual, e primal-dual barreira logarítmica. Os autores compararam as principais características desses métodos e fizeram uma revisão de suas metodologias. A comparação entre os métodos foi feita através dos seguintes critérios: perdas ativas na transmissão e geração de potência reativa na solução, número de iterações necessárias para a convergência, e tempo de processamento. As conclusões de tal estudo mostram que cada método possui vantagens e desvantagens, e, sendo assim, os autores recomendam uma abordagem para a resolução de problemas de FPO que explore qualidades de cada um desses métodos.

Sousa, Baptista e Costa (2004) apresentam uma nova abordagem para resolução de problemas de FPO através da substituição da Função Barreira Clássica (FBC) pela Função Barreira Modificada (FBM) na abordagem para resolução de problemas de FPOR proposta por Granville (1994). Nessa abordagem, a aplicação das condições necessárias de primeira ordem ao problema Lagrangiano é resolvida pelo método de Newton. Esta abordagem consiste em uma combinação das melhores propriedades dos métodos Lagrangiano e de barreira. 
Rider et al. (2004) propuseram a resolução de problemas de FPO por uma abordagem baseada na combinação dos seguintes MPIs: preditor-corretor, preditor com múltiplas correções e múltiplas correções centralizadas, pertencentes à família de MPI de alta ordem. Dessa forma, aproveitando-se as melhores propriedades de cada método, tem-se um MPI mais robusto e com características de convergência mais rápida. Segundo os autores, a ideia central dessa abordagem de MPI de alta ordem é reduzir o número de fatorações da matriz Hessiana da função Lagrangiana, que é a tarefa que exige o maior esforço computacional nos métodos clássicos de pontos interiores. Os problemas de FPO formulados foram o de minimização das perdas ativas na transmissão, do mínimo corte de carga e de máximo carregamento do sistema. Foram realizados testes com os sistemas IEEE de 30, 57, 118 e 300 barras, com o sistema interligado peruano de 464 barras e com o sistema interligado Sul-Sudeste brasileiro de 2256 barras.

Baptista et al. (2006) apresentaram uma nova abordagem para resolução de problemas de FPOR baseada na associação dos métodos de barreira e da função Lagrangiana aumentada. Nessa abordagem, o problema de FPOR é transformado em um problema irrestrito da seguinte forma: 1) as restrições canalizadas associadas aos limites das magnitudes de tensão nas barras do sistema e dos taps de transformadores em-fase são desmembradas e transformadas em restrições de igualdade pelo acréscimo de variáveis de folga não negativas; 2) a condição de não negatividade das variáveis de folga é tratada pela função barreira logarítmica, que, por sua vez, é incorporada à função objetivo do problema por um parâmetro de barreira; e 3) as demais restrições do problema são tratadas pelo método da função Lagrangiana aumentada (SANTOS JR.; DECKMANN; SOARES, 1988). O problema de barreira-Lagrangiano aumentado é resolvido pelo método de Newton. A solução do problema é encontrada quando as condições de KKT forem satisfeitas. Foram realizados testes com o sistema IEEE de 162 barras e com o sistema equivalente Sul-Sudeste brasileiro de 787 barras.

Sousa, Baptista e Costa (2009) apresentaram uma nova abordagem para a resolução de problemas de FPOR que consiste na associação dos métodos primal-dual 
barreira logarítmica e de barreira modificada. Nessa abordagem, o problema de FPOR é modificado da seguinte forma: 1) as restrições canalizadas associadas aos limites das variáveis de controle do SEE são desmembradas em desigualdades; 2) as restrições de desigualdade são transformadas em igualdades pelo acréscimo de variáveis de folga não negativas, cuja condição de não negatividade é tratada pela função barreira logarítmica modificada. Ao problema modificado, associa-se, então, uma função Lagrangiana. O problema Lagrangiano é resolvido pelo método de Newton. A relaxação da condição de não negatividade das variáveis de folga pela função barreira logarítmica modificada resulta na expansão da região factível do problema original, permitindo que a solução do problema esteja na fronteira da região factível. Foram realizados testes com os sistemas equivalentes CESP $440 \mathrm{kV}$ de 53 barras e Sul-Sudeste brasileiro de 787 barras. Um teste comparativo com o método primal-dual barreira logarítmica mostra que esta abordagem é eficiente na resolução do problema de FPOR.

\subsubsection{Variáveis de Controle Discretas}

Algumas abordagens de resolução de problemas de FPO diferenciam as variáveis de controle desse problema entre contínuas e discretas.

Liu, Papalexopoulos e Tinney (1992) propuseram um algoritmo de discretização baseado no método de penalidade para determinar o ajuste ótimo das variáveis discretas associadas a capacitores e reatores shunt em problemas de FPO. O problema de FPO é transformado em um problema irrestrito pelo método dos multiplicadores de Lagrange. As variáveis discretas são tratadas por uma função penalidade que, por sua vez, é incorporada à função Lagrangiana por um parâmetro de penalidade. Esse algoritmo consiste em resolver uma sequência de problemas Lagrangianos pelo método de Newton considerando todas as variáveis do problema contínuas. A função penalidade usada consiste em uma aproximação linear da função penalidade quadrática - devido à natureza linear dessa função, a matriz Hessiana da função Lagrangiana permanece inalterada durante as iterações do método de Newton. À medida que uma variável discreta se aproxima de um valor discreto, esta se 
torna fixa. O algoritmo converge quando todas as restrições do problema forem satisfeitas e todas as variáveis de controle discretas do problema de FPO assumirem valores discretos. Esta abordagem consiste, basicamente, em um algoritmo de "arredondamento" no decorrer das iterações do método de Newton. O sucesso desta abordagem depende de quando e como aplicar a penalidade durante as iterações do método de Newton. Foram realizados testes com sistemas elétricos de 100 e 1700 barras.

Na linha das meta-heurísticas, Bakirtzis et al. (2002) apresentaram uma abordagem baseada em algoritmos genéticos para resolução de problemas de FPO com variáveis de controle discretas. Esse algoritmo consiste em inicialmente gerar, aleatoriamente, soluções candidatas (população inicial), nas quais as variáveis de controle contínuas do sistema satisfazem seus limites, e, no caso de serem discretas, estas assumem valores discretos. Essas soluções, no entanto, podem não satisfazer às demais restrições do problema de FPO. Dessa forma, as restrições são incluídas como penalidades na função aptidão, que mede o potencial de cada solução candidata. A função aptidão designa um valor para cada solução candidata. Na resolução do problema, são realizadas as operações básicas de um algoritmo genético para as soluções candidatas: seleção, cruzamento e mutação, e, em seguida, operações avançadas e específicas para este problema. Foram realizados testes com sistemas elétricos de 30 e 76 barras. Os resultados apresentados são as melhores soluções obtidas para cada sistema após 20 gerações do algoritmo genético para cada caso. A desvantagem do uso de abordagens baseadas em algoritmos genéticos é que não há garantias de que a solução obtida seja, de fato, a solução ótima do problema. Além disso, o esforço computacional desta abordagem é muito alto, o que inviabiliza sua aplicação à resolução de problemas de FPO para sistemas elétricos de grande porte.

Liu, Tso e Cheng (2002) apresentaram uma abordagem para resolução de problemas de FPOR com variáveis contínuas e discretas para sistemas elétricos de grande porte baseada na incorporação de uma função penalidade em um algoritmo de pontos interiores primal-dual. A função penalidade apresentada nesse trabalho é quadrática, porém não é diferenciável nos pontos definidos como centros de vizinhança. Nessa 
abordagem, uma sequência de problemas de barreira-Lagrangianos é resolvida pelo método de Newton. Primeiramente o problema é resolvido assumindo-se que os parâmetros de penalidade são nulos para todas as variáveis, e estas são, então, atualizadas. Após a convergência do método de Newton, as variáveis que não assumirem valores discretos são penalizadas e as variáveis que assumirem valores discretos permanecem com o parâmetro de penalidade nulo. A introdução da penalidade no decorrer das iterações do algoritmo afeta diretamente o bom desempenho desta abordagem. Se a penalidade for usada antecipadamente, a função objetivo é perturbada e, assim, obtém-se soluções não ótimas. Se a penalidade for introduzida quando o algoritmo já convergiu para a solução contínua, mais iterações do algoritmo serão necessárias para que se obtenha uma solução discreta. Foram realizados testes com sistemas elétricos de 14, 30, 68, 118 e 538 barras. Os resultados mostram que o algoritmo encontra soluções discretas de boa qualidade em um tempo computacional aceitável.

Ding, Wang e Song (2004) propuseram uma abordagem de método de planos de corte para resolver problemas de FPO com taps dos transformadores em-fase modelados como variáveis de controle discretas. O método resolve sucessivas linearizações do problema de FPO. Nos métodos de planos de cortes tradicionais, esses problemas de PL são resolvidos pelo método Simplex. Nesta abordagem, esses problemas são resolvidos por um MPI. Primeiramente, o método resolve o problema de FPO com as variáveis discretas fixas, obtendo-se, assim, uma solução inicial factível. O problema de FPO é então linearizado na vizinhança desta solução inicial, obtendo-se, assim, um problema de PL. Se este problema for infactível, o processo é finalizado. Caso contrário, o FPO linearizado é resolvido pelo MPI. Se na solução do problema de FPO linearizado algum tap assumir um valor discreto, essa variável é fixa. Caso contrário, um plano de corte é gerado, definindo-se, assim, um novo problema de PL. Planos de cortes são gerados e adicionados às restrições do problema de FPO linearizado até que uma solução com valores discretos para todos os taps seja obtida. A cada vez que um tap assumir um valor discreto na solução do FPO linearizado, o problema de FPO é resolvido com estas variáveis fixas, e as demais etapas do processo 
são repetidas. Nesse trabalho são detalhados os procedimentos adotados para gerar os planos de cortes. Foram realizados testes com os sistemas IEEE de 14, 57, 118 e 300 barras. Os testes mostraram que esta abordagem é mais rápida que os métodos de planos de cortes que fazem uso do método Simplex na resolução dos problemas de PL. Para os sistemas de 14, 57 e 118 barras, o problema foi resolvido em baixo tempo computacional. Para o sistema de 300 barras, o tempo de processamento foi alto.

Lin, Ho e Lin (2004) apresentaram um algoritmo que fornece uma solução suficientemente boa para o problema de FPO com variáveis de controle contínuas e discretas. O objetivo deste algoritmo é determinar uma solução discreta satisfatória em baixo tempo computacional, porém, não necessariamente uma solução ótima, superando as metodologias meta-heurísticas em tempo de processamento. O algoritmo é baseado em um procedimento de busca. Primeiramente, as variáveis de controle discretas são consideradas contínuas e o problema de FPO é resolvido. O conjunto das soluções candidatas é constituído por todas as combinações possíveis de "arredondamento" de cada variável para o valor discreto mais próximo, inferior e superior. Em seguida, esse conjunto das soluções candidatas é reduzido utilizando-se uma heurística baseada na análise de sensibilidade e um modelo simplificado do problema de FPO. As soluções mais promissoras são avaliadas através do modelo exato do problema de FPO e assim, segundo os autores, obtém-se uma solução suficientemente boa para o problema de FPO com controles discretos. Foram realizados testes com o sistema IEEE de 118 barras e com um sistema de 244 barras. A desvantagem desta abordagem é que a solução obtida não é uma solução ótima - esta é apenas a melhor solução entre as combinações das soluções "arredondadas".

Capitanescu e Wehenkel (2010) apresentaram três abordagens para resolução de problemas de FPO com variáveis de controle discretas. A primeira delas consiste em resolver o problema de FPO com todas variáveis consideradas contínuas e, então, no ponto obtido, determinar a matriz sensibilidade da função Lagrangiana em relação às variáveis discretas. Essas informações são incorporadas a um problema de Programação Linear Inteira-Mista (PLIM) cuja solução fornece os ajustes das variáveis de controle 
discretas do problema de FPO. Em seguida, o problema de FPO com as variáveis discretas fixas é resolvido e, com isso, obtém-se os ajustes ótimos das variáveis de controle contínuas. Para a resolução do problema de FPO, utiliza-se um MPI, e para a resolução do PLIM, utiliza-se um algoritmo branch-and-cut. A segunda abordagem é análoga à primeira, porém o problema de PLIM usado na determinação dos ajustes das variáveis de controle discretas é substituído por um procedimento baseado em uma função mérito. Já a terceira abordagem explora os multiplicadores de Lagrange associados aos limites das variáveis de controle discretas. Foram realizados testes com o sistema IEEE de 300 barras e com sistemas reais de 60, 618 e 1203 barras. Os testes realizados com as abordagens de resolução propostas foram comparados à abordagem de "arredondamento". Os resultados mostram que a segunda abordagem proposta é superior às demais. Os três procedimentos consistem em métodos iterativos que fixam valores para as variáveis de controle discretas, valores estes determinados por métodos heurísticos, e então resolve-se o problema de FPO em função das variáveis de controle contínuas. Essas heurísticas apresentadas exploram apenas a vizinhança dos valores discretos da solução fornecida pelo algoritmo.

\subsubsection{Restrições de Complementaridade}

Rosehart, Roman e Schellenberg (2005) propuseram a modelagem, por restrições de complementaridade, do controle da magnitude de tensão pela geração de potência reativa. As restrições de complementaridade modelam a "troca" de variáveis de controle para variável dependente quando limites de geração de potência reativa são atingidos. Essas restrições são, então, incorporadas no conjunto de restrições dos problemas de máximo carregamento e de FPO com restrição de estabilidade de tensão, os quais foram resolvidos por um algoritmo de pontos interiores primal-dual. Foram realizados testes com os sistemas IEEE de 57 e 300 barras. Os resultados mostram que essa modelagem do controle da magnitude de tensão tem grande influência na determinação do ajuste ótimo das variáveis de controle de um SEE na solução de problemas de FPO. 
Bautista, Anjos e Vannelli (2007) apresentaram uma formulação de dois níveis do problema de despacho ativo e reativo para análise da influência das restrições de magnitude de tensão e de geração de potência reativa na definição de estratégias de oferta de energia no modelo de competição imperfeita supply function equilibrium (modelo de equilíbrio de funções de oferta). O problema do segundo nível consiste em um problema de FPO clássico, cujo critério a ser minimizado é o benefício comum. Para resolver esse problema de Programação Binível (PB), o problema de FPO do segundo nível foi transformado em um problema de complementaridade não linear misto pela aplicação das condições de KKT e incorporado no conjunto das restrições do problema do primeiro nível, resultando em um problema de PNL. As restrições de folga complementar de KKT foram tratadas de três formas distintas: pela função Fischer-Burmeister, pela transformação em restrições de desigualdade equivalentes, e por penalidade. Destas, a abordagem que obteve melhor desempenho computacional foi a pela transformação das restrições de complementaridade em restrições de desigualdade equivalentes. Foram realizados testes com um sistema de 3 barras e com o sistema IEEE de 14 barras.

Almeida e Senna (2011) apresentaram uma formulação de dois níveis do problema de despacho ativo e reativo para minimizar custo de oportunidade associado à geração de potência reativa. O problema do segundo nível consiste minimizar o preço da energia para os consumidores, considerando o modelo não linear da rede elétrica. Para resolver esse problema de PB, o problema de FPO do segundo nível foi transformado em um problema de complementaridade não linear misto pela aplicação das condições de KKT e incorporado no conjunto das restrições do problema do primeiro nível, resultando em um problema de PNL. As restrições de folga complementar de KKT foram tratadas pela função de Fischer-Burmeister modificada, suavizada por um parâmetro que tende a zero. O problema de PNL resultante foi resolvido por um método de pontos interiores primal-dual modificado para o tratamento de restrições de complementaridade. Foram realizados testes com um sistema de 2 barras, com o sistema IEEE de 30 barras e com o sistema equivalente Sul brasileiro de 352 barras. 


\section{Capítulo 3}

\section{Métodos de Otimização}

Métodos de otimização consistem na determinação sistemática do ponto de mínimo ou máximo de uma determinada função em um subespaço do seu domínio.

Matemáticos têm trabalhado no desenvolvimento de métodos de otimização desde as proposições de Fermat, no século XVII, para o traçado de tangentes e a determinação de pontos extremos de curvas, antes mesmo do desenvolvimento das bases do cálculo diferencial por Newton. No entanto, até a década de 1940, relativamente muito pouco havia sido desenvolvido para a otimização de funções de muitas variáveis.

Com o surgimento do computador, na década de 1940, métodos de otimização para problemas de grande porte puderam ser, então, desenvolvidos. Entre os métodos de PL, que buscam otimizar uma função objetivo linear sujeita a restrições também lineares, destaca-se o método Simplex desenvolvido por Dantzig, em 1947, cuja principal motivação foi acelerar o processo de cálculo de alocação de recursos da Força Aérea Americana no período pós-Segunda Guerra Mundial. Desde então, graças ao surgimento de computadores cada vez mais velozes e ao desenvolvimento de algoritmos e programas computacionais eficientes e robustos, o bom desempenho da representação de problemas de otimização por meio da PL fazem da mesma uma ferramenta de extrema importância para a análise de vários problemas de engenharia, administração, logística, transporte, economia, química, biologia etc. 
Entretanto, muitos problemas reais não podem ser analisados adequadamente, nem sequer aproximados, por um problema de PL devido à natureza não linear de sua função objetivo e/ou suas restrições. Nesses casos, tais problemas podem ser resolvidos por métodos de PNL ou meta-heurísticos.

A PNL não possui um método geral para resolução de seus problemas. O desenvolvimento de novas abordagens para resolução desses problemas se baseia, basicamente, na associação de métodos clássicos de PNL que exploram características específicas dos problemas aos quais são aplicados. Dessa forma, neste capítulo, apresenta-se uma revisão dos métodos de otimização que dão suporte teórico para o desenvolvimento da abordagem para resolução de problemas de PNL apresentada neste trabalho. São apresentados os métodos dual-Lagrangiano, de Newton-Lagrangiano, de penalidade, de barreira e de barreira modificada, os quais, por si só, permitem a resolução de um grande número de problemas de PNL.

\subsection{Método Dual-Lagrangiano}

Considere o seguinte problema de PNL, denominado problema primal:

$$
\begin{array}{lll}
\min & f(x) & \\
\text { s.a: } & g_{i}(x)=0 & i=1, \cdots, p \\
& h_{i}(x) \leq 0 & i=1, \cdots, q
\end{array}
$$

onde $x \in \mathcal{X} \subseteq \mathbb{R}^{n} ; f: \mathbb{R}^{n} \rightarrow \mathbb{R} ; g: \mathbb{R}^{n} \rightarrow \mathbb{R}^{p}$, com $p<n$; e $h: \mathbb{R}^{n} \rightarrow \mathbb{R}^{q}$. Considere também que as funções $f, g$ e $h$ sejam de classe $C^{2}$.

O problema dual-Lagrangiano associado ao problema primal (3.1) é definido como:

$$
\begin{array}{cl}
\max & \theta(\lambda, \pi) \\
\text { s.a: } & \pi_{i} \geq 0 \quad i=1, \cdots, q
\end{array}
$$


onde $\lambda \in \mathbb{R}^{p}, \pi \in \mathbb{R}^{q}$ e a função $\theta$ é dada por:

$$
\theta(\lambda, \pi)=\min _{x}\left\{f(x)+\sum_{i=1}^{p} \lambda_{i} g_{i}(x)+\sum_{i=1}^{q} \pi_{i} h_{i}(x): x \in \mathcal{X}\right\}
$$

O problema dual-Lagrangiano também é conhecido como max-min $\mathcal{L}(x, \lambda, \pi)$, onde $\mathcal{L}$ é denominada função Lagrangiana:

$$
\mathcal{L}(x, \lambda, \pi)=f(x)+\sum_{i=1}^{p} \lambda_{i} g_{i}(x)+\sum_{i=1}^{q} \pi_{i} h_{i}(x)
$$

A função Lagrangiana $\mathcal{L}$ consiste, portanto, na incorporação de uma combinação das restrições $g$ e $h$ na função objetivo do problema primal pelos multiplicadores de Lagrange $\lambda$ e $\pi$, respectivamente.

A relação entre o problema primal e o dual-Lagrangiano pode ser melhor explicada através do enunciado do seguinte teorema (BAZARAA, 1993):

Teorema (Teorema do Ponto de Sela). Seja $\mathcal{X}$ um conjunto não vazio em $\mathbb{R}^{n}$, e seja $f: \mathbb{R}^{n} \rightarrow \mathbb{R}, g: \mathbb{R}^{n} \rightarrow \mathbb{R}^{p}$, e $h: \mathbb{R}^{n} \rightarrow \mathbb{R}^{q}$. Suponha que exista $x^{*} \in \mathcal{X}$ e $\left(\lambda^{*}, \pi^{*}\right)$ com $\pi_{i}^{*} \geq 0$ para $i=1, \cdots, q$ tal que:

$$
\mathcal{L}\left(x^{*}, \lambda, \pi\right) \leq \mathcal{L}\left(x^{*}, \lambda^{*}, \pi^{*}\right) \leq \mathcal{L}\left(x, \lambda^{*}, \pi^{*}\right)
$$

para todo $x \in \mathcal{X}$ e todo $(\lambda, \pi)$ com $\pi_{i} \geq 0$ para $i=1, \cdots, q$. Portanto, $x^{*}$ e $\left(\lambda^{*}, \pi^{*}\right)$ são soluções, respectivamente, dos problemas primal e dual.

Dessa forma, se o problema (3.1) for convexo, existem multiplicadores $\lambda^{*}$ e $\pi^{*}$ que, aplicados ao problema irrestrito:

$$
\min _{x} \mathcal{L}\left(x, \lambda^{*}, \pi^{*}\right)
$$

fazem com que a solução de (3.4) coincida com a solução de (3.1). O problema (3.4) é denominado problema Lagrangiano. 


\subsubsection{Algoritmo}

1) Dado o problema (3.1), construa a função Lagrangiana(3.2);

2) Faça $k=0$;

Dê uma estimativa inicial para $x^{0} \in \mathcal{X}$ e para os multiplicadores de Lagrange $\lambda^{0} \mathrm{e}$ $\pi^{0}, \operatorname{com} \pi_{i}^{0} \geq 0$ para $i=1, \cdots, q$;

3) Resolva o problema Lagrangiano (3.4), com $\lambda^{k}$ e $\pi^{k}$ fixos, utilizando um método de otimização irrestrita:

$$
\min _{x} f\left(x^{k}\right)+\sum_{i=1}^{p} \lambda_{i}^{k} g_{i}\left(x^{k}\right)+\sum_{i=1}^{q} \pi_{i}^{k} h_{i}\left(x^{k}\right)
$$

4) Admita $x^{k+1} \in \mathcal{X}$ como uma solução e vá para o passo 5 ;

5) Se as condições de KKT forem satisfeitas para $x^{k+1}, \lambda^{k}$ e $\pi^{k}$, pare.

Caso contrário, atualize os multiplicadores de Lagrange utilizando uma heurística, determinando $\lambda^{k+1}$ e $\pi^{k+1}$, faça $k=k+1$, e volte para o passo 3 .

Vários algoritmos podem ser utilizados para gerar estimativas e atualizar os multiplicadores de Lagrange $\lambda$ e $\pi$. Uma escolha é o algoritmo do gradiente, que gera uma sequência de correções para os multiplicadores $\lambda$ e $\pi$ pela seguinte expressão:

$$
\left[\begin{array}{c}
\lambda^{k+1} \\
\pi^{k+1}
\end{array}\right]=\left[\begin{array}{c}
\lambda^{k} \\
\pi^{k}
\end{array}\right]+\alpha\left[\begin{array}{c}
g\left(x^{k}\right) \\
s
\end{array}\right]
$$

onde $\alpha$ é determinado por um processo de busca unidimensional e $s$ é dado por:

$$
s_{i}=\left\{\begin{array}{lll}
h_{i}\left(x^{k}\right) & \text { se } & \pi_{i}^{k}>0 \\
\max \left\{0, h_{i}\left(x^{k}\right)\right\} & \text { se } & \pi_{i}^{k}=0
\end{array}\right.
$$

para $i=1, \cdots, q$. 


\subsubsection{Dificuldades Computacionais}

Para problemas não convexos em torno da solução, pode ocorrer a existência do gap de dualidade e, portanto, o método dual-Lagrangiano não obtém a solução ótima do problema. Uma outra desvantagem deste método é o aumento do número de variáveis do problema.

\subsection{Método de Newton-Lagrangiano}

Considere o seguinte problema de PNL:

$$
\begin{array}{ll}
\min & f(x) \\
\text { s.a: } & g_{i}(x)=0 \quad i=1, \cdots, p
\end{array}
$$

onde $x \in \mathcal{X} \subseteq \mathbb{R}^{n} ; f: \mathbb{R}^{n} \rightarrow \mathbb{R} ;$ e $g: \mathbb{R}^{n} \rightarrow \mathbb{R}^{p}$, com $p<n$. Considere também que as funções $f$ e $g$ sejam de classe $C^{2}$.

O problema Lagrangiano associado ao problema (3.7) é definido como:

$$
\max _{\lambda} \min _{x} \mathcal{L}(x, \lambda)
$$

onde a função Lagrangiana $\mathcal{L}$ é dada por:

$$
\mathcal{L}(x, \lambda)=f(x)+\sum_{i=1}^{p} \lambda_{i} g_{i}(x)
$$

O método de Newton-Lagrangiano consiste em determinar, simultaneamente, valores para $x$ e $\lambda$ que satisfaçam as condições necessárias de otimalidade do problema 
Lagrangiano (3.8), ou seja:

$$
\begin{aligned}
& \nabla_{x} \mathcal{L}(x, \lambda)=0 \\
& \nabla_{\lambda} \mathcal{L}(x, \lambda)=0
\end{aligned}
$$

Para determinar a solução do sistema de equações não lineares (3.10), emprega-se método de Newton. Portanto, através da expansão de (3.10) por Taylor de primeira ordem em torno de $x^{k}$ e $\lambda^{k}$, obtém-se o seguinte sistema de equações lineares:

$$
\begin{aligned}
& \nabla_{x} \mathcal{L}\left(x^{k}, \lambda^{k}\right)+\nabla_{x x}^{2} \mathcal{L}\left(x^{k}, \lambda^{k}\right) \Delta x^{k}+\nabla_{x \lambda}^{2} \mathcal{L}\left(x^{k}, \lambda^{k}\right) \Delta \lambda^{k}=0 \\
& \nabla_{\lambda} \mathcal{L}\left(x^{k}, \lambda^{k}\right)+\nabla_{\lambda x}^{2} \mathcal{L}\left(x^{k}, \lambda^{k}\right) \Delta x^{k}+\nabla_{\lambda \lambda} \mathcal{L}\left(x^{k}, \lambda^{k}\right) \Delta \lambda^{k}=0
\end{aligned}
$$

ou, de forma equivalente:

$$
\begin{aligned}
& \nabla_{x} \mathcal{L}\left(x^{k}, \lambda^{k}\right)+\nabla_{x x}^{2} \mathcal{L}\left(x^{k}, \lambda^{k}\right) \Delta x^{k}+\nabla_{x} g\left(x^{k}\right)^{T} \Delta \lambda^{k}=0 \\
& g\left(x^{k}\right)+\nabla_{x} g\left(x^{k}\right) \Delta x^{k}=0
\end{aligned}
$$

O sistema (3.12) pode, ainda, ser representado na forma matricial:

$$
\left[\begin{array}{cc}
\nabla_{x x}^{2} \mathcal{L}\left(x^{k}, \lambda^{k}\right) & \nabla_{x} g\left(x^{k}, \lambda^{k}\right)^{T} \\
\nabla_{x} g\left(x^{k}, \lambda^{k}\right) & 0
\end{array}\right]\left[\begin{array}{c}
\Delta x^{k} \\
\Delta \lambda^{k}
\end{array}\right]=-\left[\begin{array}{c}
\nabla_{x} \mathcal{L}\left(x^{k}, \lambda^{k}\right) \\
g\left(x^{k}\right)
\end{array}\right]
$$

ou, de forma simplificada:

$$
\left[\begin{array}{cc}
H & J_{g}^{T} \\
J_{g} & 0
\end{array}\right]\left[\begin{array}{c}
\Delta x^{k} \\
\Delta \lambda^{k}
\end{array}\right]=-\left[\begin{array}{c}
\nabla_{x} \mathcal{L} \\
\nabla_{\lambda} \mathcal{L}
\end{array}\right]
$$

onde a matriz dos coeficientes é denominada matriz Hessiana da função Lagrangiana, e encontra-se dividida em quatro sub-matrizes: uma matriz Hessiana $H=\nabla_{x x}^{2} \mathcal{L}(x, \lambda)$, uma matriz Jacobiana $J_{g}=\nabla_{x} g(x)$ e sua transposta, e uma matriz nula. 
A nova estimativa da solução de (3.10) é dada por:

$$
\begin{aligned}
& x^{k+1}=x^{k}+\Delta x^{k} \\
& \lambda^{k+1}=\lambda^{k}+\Delta \lambda^{k}
\end{aligned}
$$

onde $\Delta x \in \mathbb{R}^{n}$ e $\Delta \lambda \in \mathbb{R}^{p}$ são denominados vetores de direção de busca.

A solução do problema (3.7) é determinada quando os novos valores de $x$ e $\lambda$ satisfizerem as condições de KKT para o problema original.

\subsubsection{Algoritmo}

1) Dado o problema (3.7), construa a função Lagrangiana (3.9);

2) Faça $k=0$;

Dê uma estimativa inicial para $x^{0} \in \mathcal{X}$ e para os multiplicadores de Lagrange $\lambda^{0}$;

3) Se $x^{k}$ e $\lambda^{k}$ satisfizerem as condições de KKT, pare;

Caso contrário, vá para o passo 4;

4) Resolva o sistema de equações (3.14), atualize as variáveis $x$ e $\lambda$ por (3.15), faça $k=k+1$ e volte para o passo 3 .

\subsubsection{Dificuldades Computacionais}

O método de Newton-Lagrangiano exige que a estimativa inicial da solução do problema esteja na vizinhança de $x^{*}$ e $\lambda^{*}$, o que, muitas vezes, pode inviabilizar seu uso, pois não existe garantia de convergência. 


\subsection{Método de Penalidade}

Considere o seguinte problema de PNL:

$$
\begin{array}{lll}
\min & f(x) & \\
\text { s.a: } & g_{i}(x)=0 & i=1, \cdots, p \\
& h_{i}(x) \leq 0 & i=1, \cdots, q
\end{array}
$$

onde $x \in \mathcal{X} \subseteq \mathbb{R}^{n} ; f: \mathbb{R}^{n} \rightarrow \mathbb{R} ; g: \mathbb{R}^{n} \rightarrow \mathbb{R}^{p}$, com $p<n$; e $h: \mathbb{R}^{n} \rightarrow \mathbb{R}^{q}$. Considere também que as funções $f, g$ e $h$ sejam de classe $C^{2}$.

A estratégia dos métodos de penalidade é a utilização de uma função auxiliar onde as restrições são incorporadas na função objetivo por termos de penalidade, os quais penalizam qualquer violação destas. Esses métodos geram uma sequência de pontos infactíveis, até convergirem para a solução do problema (3.16).

A função auxiliar tem a forma $f(x)+\omega \mathcal{P}(x)$, sendo $\omega$ o parâmetro de penalidade e $\mathcal{P}$ a função penalidade associada a (3.16), dada por:

$$
\mathcal{P}(x)=\sum_{i=1}^{p} \alpha\left(g_{i}(x)\right)+\sum_{i=1}^{q} \varphi\left(h_{i}(x)\right)
$$

onde $\alpha: \mathbb{R} \rightarrow \mathbb{R}$ e $\varphi: \mathbb{R} \rightarrow \mathbb{R}$ são funções contínuas em $y \in \mathbb{R}$, tais que:

$$
\begin{array}{llllll}
\alpha(y)=0 & \text { se } \quad y=0 & \text { e } & \alpha(y)>0 & \text { se } & y \neq 0 \\
\varphi(y)=0 & \text { se } \quad y \leq 0 & \text { e } & \varphi(y)>0 & \text { se } & y>0
\end{array}
$$


As funções $\alpha$ e $\varphi$ podem assumir as seguintes formas:

$$
\begin{aligned}
& \alpha(y)=|y|^{\beta} \\
& \varphi(y)=(\max \{0 ; y\})^{\beta}
\end{aligned}
$$

onde $\beta$ é um inteiro positivo. Para $\beta=2$, a função $\mathcal{P}$ é denominada função penalidade quadrática.

O problema de penalidade consiste, portanto, no seguinte problema irrestrito:

$$
\min _{x} \quad f(x)+\omega \mathcal{P}(x)
$$

onde $\omega>0$ e $x \in \mathcal{X}$.

Caso $\omega \mathcal{P}(x)$ não seja menor que uma tolerância de convergência $\xi$ especificada, o parâmetro $\omega$ é atualizado por um fator $\rho>1$, e o problema (3.18) é resolvido novamente.

À medida que $\omega \rightarrow \infty$ e $\mathcal{P}(x) \rightarrow 0$, a solução do problema (3.18) converge para a solução do problema (3.16).

\subsubsection{Algoritmo}

1) Dado o problema (3.16), construa a função penalidade (3.17);

2) Faça $k=0$;

Dê um valor inicial para $x^{0} \in \mathcal{X}$ e para o parâmetro $\omega^{0}>0$;

Especifique um valor para o fator $\rho>1$ e uma tolerância $\xi>0$;

3) Resolva o problema de penalidade (3.18), com $\omega^{k}$ fixo, utilizando um método de otimização irrestrita:

$$
\min _{x} \quad f\left(x^{k}\right)+\omega^{k} \mathcal{P}\left(x^{k}\right)
$$


4) Admita $x^{k+1} \in \mathcal{X}$ como uma solução e vá para o passo 5;

5) $\operatorname{Se} \omega^{k} \mathcal{P}\left(x^{k+1}\right) \leq \xi$, pare.

Caso contrário, atualize o parâmetro de penalidade por $\omega^{k+1}=\rho \omega^{k}$; faça $k=k+1$ e volte para o passo 3 .

\subsubsection{Dificuldades Computacionais}

Para $\omega$ suficientemente grande, a solução do problema de penalidade será próxima à solução do problema original. Entretanto, para valores muito grandes de $\omega$, a matriz Hessiana pode se tornar mal condicionada. Para valores muito grandes de $\omega^{0}$ e do fator $\rho$, há uma maior ênfase sobre a factibilidade e a maioria dos métodos de otimização irrestrita move-se rapidamente na direção de um ponto factível, porém não ótimo, causando um término prematuro do método. Dessa forma, as escolhas do valor inicial de $\omega$ e do fator $\rho$ podem comprometer o processo de otimização.

\subsection{Método de Barreira}

Considere o seguinte problema de PNL:

$$
\begin{array}{ll}
\min & f(x) \\
\text { s.a: } & h_{i}(x) \geq 0 \quad i=1, \cdots, q
\end{array}
$$

onde $x \in \mathcal{X} \subseteq \mathbb{R}^{n} ; f: \mathbb{R}^{n} \rightarrow \mathbb{R} ;$ e $h: \mathbb{R}^{n} \rightarrow \mathbb{R}^{q}$. Considere também que as funções $f$ e $h$ sejam de classe $C^{2}$.

Da mesma forma que os métodos de penalidade, os métodos de barreira transformam o problema (3.19) em um problema irrestrito. Neles, as restrições de desigualdade são incorporadas na função objetivo por termos de barreira, definindo uma função auxiliar. Trabalhando no interior da região factível do problema original, tais termos criam barreiras que impedem que as restrições de desigualdade tenham seus limites 
violados. Esses métodos só trabalham com problemas cujo interior da região factível é não vazio, e uma de suas vantagens é a obtenção de, pelo menos, uma solução factível para o problema primal caso ocorra uma parada prematura do mesmo.

A função auxiliar tem a forma $f(x)+\mu \mathcal{B}(x)$, sendo $\mu$ o parâmetro de barreira e $\mathcal{B}$ a função barreira associada a (3.19), dada por:

$$
\mathcal{B}(x)=\sum_{i=1}^{q} \phi\left(h_{i}(x)\right)
$$

onde $\phi: \mathbb{R} \rightarrow \mathbb{R}$ é uma função contínua em $\{y: y>0\}$, tal que:

$$
\phi(y) \geq 0 \quad \text { se } \quad y>0 \quad \text { e } \quad \lim _{y \rightarrow 0^{+}} \phi(y)=\infty
$$

A função $\phi$ pode assumir várias formas, como, por exemplo:

$$
\begin{aligned}
& \phi(y)=\frac{1}{y} \\
& \phi(y)=-\ln (\min \{1, y\})
\end{aligned}
$$

Observe que (3.23) não é diferenciável em $\{y: y>0\}$ devido ao termo $\min \{1, y\}$. No entanto, como as condições definidas em (3.21) são essenciais somente na vizinhança de $y=0,(3.23)$ pode assumir a seguinte forma:

$$
\phi(y)=-\ln (y)
$$

A função (3.22) é denominada função barreira inversa (CARROL, 1961). A função (3.24) é denominada função barreira logarítmica (FRISCH, 1955).

A função $\mathcal{B}$ é uma função não negativa e contínua em $\mathcal{X}^{\prime}=\{x: h(x)>0\}$ e tende ao infinito à medida que a solução se aproxima da fronteira definida por $h(x)=0$, a partir do interior. 
O problema de barreira é dado por:

$$
\min _{x} \quad\{f(x)+\mu \mathcal{B}(x): h(x)>0\}
$$

onde $\mu>0$ e $x \in \mathcal{X}^{\prime} \subset \mathcal{X}$.

Quando $\mu \rightarrow 0$ e $\mathcal{B}(x) \rightarrow \infty$, tem-se que $\mu \mathcal{B}(x)$ se aproxima da função barreira ideal, e a solução do problema (3.25) converge para a solução do problema (3.19).

Caso $\mu \mathcal{B}(x)$ não seja menor que uma tolerância de convergência $\xi$ especificada, o parâmetro $\mu$ é atualizado por um fator $\tau$, tal que $0<\tau<1$, e o problema (3.25) é resolvido novamente. Durante o processo de resolução de (3.25), os ajustes nas variáveis $x$ devem ser monitorados a cada iteração para que a condição $h(x)>0$ não seja violada.

\subsubsection{Algoritmo}

1) Dado o problema (3.19), somente com restrições de desigualdade, construa a função barreira (3.20);

2) Faça $k=0$;

Dê um valor inicial para $x^{0} \in \mathcal{X}^{\prime}$ e para o parâmetro $\mu^{0}>0$;

Especifique um valor para o fator $\tau$, tal que $0<\tau<1$, e uma tolerância $\xi>0$;

3) Resolva o problema de barreira (3.25), com $\mu^{k}$ fixo, utilizando um método de otimização irrestrita:

$$
\min _{x} \quad\left\{f\left(x^{k}\right)+\mu^{k} \mathcal{B}\left(x^{k}\right): h\left(x^{k}\right)>0\right\}
$$

4) Admita $x^{k+1} \in \mathcal{X}^{\prime}$ como uma solução e vá para o passo 5;

5) $\operatorname{Se} \mu^{k} \mathcal{B}\left(x^{k+1}\right) \leq \xi$, pare.

Caso contrário, atualize o parâmetro de barreira por $\mu^{k+1}=\tau \mu^{k}$, faça $k=k+1$, e volte para o passo 3 . 


\subsubsection{Dificuldades Computacionais}

Uma das dificuldades encontradas nos métodos de barreira é a seleção de um ponto inicial $x$ factível. Para muitos problemas, isso pode ser trabalhoso. Também, em virtude da estrutura da função barreira, para valores pequenos de $\mu$, muitas técnicas encontram sérios problemas de mal condicionamento e erros de arredondamento à medida que a solução se aproxima do ótimo. As escolhas do valor inicial de $\mu$ e do fator $\tau$ podem comprometer o processo de otimização.

\subsection{Método de Barreira Modificada}

Considere o seguinte problema de PNL:

$$
\begin{array}{ll}
\min & f(x) \\
\text { s.a: } & h_{i}(x) \geq 0 \quad i=1, \cdots, q
\end{array}
$$

onde $x \in \mathcal{X} \subseteq \mathbb{R}^{n} ; f: \mathbb{R}^{n} \rightarrow \mathbb{R} ;$ e $h: \mathbb{R}^{n} \rightarrow \mathbb{R}^{q}$. Considere também que as funções $f$ e $h$ sejam de classe $C^{2}$.

Polyak desenvolveu, em 1992, uma teoria de métodos de barreira modificada para transformar o problema (3.26) em um problema irrestrito. Neles, as restrições de desigualdade são relaxadas por um parâmetro $\mu$ e introduzidas na função objetivo por uma combinação da função Lagrangiana clássica e da função barreira clássica, explorando as melhores propriedades de cada uma dessas funções; a função barreira modificada pode ser considerada como uma função Lagrangiana aumentada interior.

O problema de barreira modificada consiste no seguinte problema irrestrito:

$$
\min _{x} \quad\{\mathcal{M}(x, \sigma): h(x)>-\mu\}
$$


onde $\sigma \in \mathbb{R}^{q}$, com $\sigma_{i} \geq 0$ para $i=1, \cdots, q$, são estimativas dos multiplicadores de Lagrange associados às restrições de desigualdade relaxadas; $\mu>0$ é o parâmetro de barreira modificada; e $\mathcal{M}$ é a função barreira modificada associada ao problema (3.26):

$$
\mathcal{M}(x, \sigma)=f(x)-\mu \sum_{i=1}^{q} \sigma_{i} \psi\left(\mu^{-1} h_{i}(x)+1\right)
$$

A função $\psi$ em (3.28) representa o termo de barreira. Essa função é estritamente côncava, de classe $C^{2}$ e definida no intervalo $(0 ;+\infty)$.

A função $\mathcal{M}$ consiste, portanto, na função Lagrangiana clássica associada ao problema (3.26) com as restrições de desigualdade modificadas pelo termo de barreira $\psi$. Os termos de barreira $\psi\left(\mu^{-1} h_{i}(x)+1\right)$, para $i=1, \cdots, q$, correspondem à relaxação $h_{i}(x) \geq-\mu$. Essa relaxação representa uma expansão da região factível do problema (3.26). Consequentemente, a região factível do problema (3.27) varia em função do parâmetro $\mu$.

A função $\mathcal{M}$ é uma função contínua em $\mathcal{X}^{\prime}=\{x: h(x)>-\mu\}$ e tende ao infinito à medida que a solução se aproxima da fronteira definida por $h(x)=-\mu$, a partir do interior.

De acordo com Polyak (1992), as funções barreira modificada de Frisch e de Carrol são dadas, respectivamente, por:

$$
\begin{aligned}
& \mathcal{F}(x, \sigma)= \begin{cases}f(x)-\mu \sum_{i=1}^{q} \sigma_{i} \ln \left(\mu^{-1} h_{i}(x)+1\right) & \text { se } x \in \mathcal{X}^{\prime} \\
+\infty & \text { se } \quad x \notin \mathcal{X}^{\prime}\end{cases} \\
& \mathcal{C}(x, \sigma)=\left\{\begin{array}{lll}
f(x)+\mu \sum_{i=1}^{q} \sigma_{i}\left[\left(\mu^{-1} h_{i}(x)+1\right)^{-1}-1\right] & \text { se } x \in \mathcal{X}^{\prime} \\
+\infty & \text { se } x \notin \mathcal{X}^{\prime}
\end{array}\right.
\end{aligned}
$$

Diferentemente das funções barreira, a função $\mathcal{M}$ e suas derivadas de primeira e segunda ordem são definidas na fronteira da região factível do problema (3.26) para 
qualquer $\mu>0$. Em particular, se $x^{*}$ e $\sigma^{*}$ forem conhecidos, a função $\mathcal{M}$ possui as seguintes propriedades para qualquer $\mu>0$ (POLYAK, 1992):

P1. $\mathcal{M}\left(x^{*}, \sigma^{*}\right)=f\left(x^{*}\right)$

P2. $\nabla_{x} \mathcal{M}\left(x^{*}, \sigma^{*}\right)=\nabla_{x} f\left(x^{*}\right)-\sum_{i=1}^{q} \sigma_{i}^{*} \nabla_{x} h_{i}\left(x^{*}\right)=0$

Se o problema (3.26) for convexo, segue de P2 que:

P3. $x^{*}=\arg \min \left\{\mathcal{M}\left(x, \sigma^{*}\right)\right\}$ para qualquer $\mu>0$.

Isso significa que, se os multiplicadores de Lagrange $\sigma^{*}$ forem conhecidos, o problema de barreira modificada pode ser resolvido independentemente do valor de $\mu$ (POLYAK, 1992). Como o parâmetro $\mu$ não precisa tender a zero para que o método alcance a solução do problema (desde que se tenha uma boa estimativa dos multiplicadores $\sigma$ ), o condicionamento da matriz Hessiana é fortemente melhorado.

O algoritmo da barreira modificada possui uma característica de convergência finita, o que não acontece nos métodos de barreira. Tal característica torna explícito o uso dos multiplicadores de Lagrange $\sigma$.

\subsubsection{Algoritmo}

1) Dado o problema (3.26), somente com restrições de desigualdade, construa a função barreira (3.28);

2) Faça $k=0$;

Dê um valor inicial para $x^{0} \in \mathcal{X}^{\prime}$ e para o parâmetro $\mu^{0}>0$;

Dê uma estimativa para os multiplicadores de Lagrange $\sigma^{0}$;

Especifique um valor para o fator $\tau$, tal que $0<\tau<1$, e uma tolerância $\xi>0$; 
3) Resolva o problema de barreira (3.27), com $\sigma^{k}$ e $\mu^{k}$ fixos, utilizando um método de otimização irrestrita:

$$
\min _{x}\left\{f\left(x^{k}\right)-\mu^{k} \sum_{i=1}^{q} \sigma_{i}^{k} \psi\left(\left(\mu^{k}\right)^{-1} h_{i}\left(x^{k}\right)+1\right): h\left(x^{k}\right)>-\mu^{k}\right\}
$$

4) Admita $x^{k+1} \in \mathcal{X}^{\prime}$ como uma solução e vá para o passo 5;

5) Se as condições de KKT forem satisfeitas para $x^{k+1}$ e $\sigma^{k}$ e se $h\left(x^{k}\right) \geq 0$, pare.

Caso contrário, atualize os multiplicadores de Lagrange, determinando $\sigma^{k+1}$, e o parâmetro de barreira modificada por $\mu^{k+1}=\tau \mu^{k}$, faça $k=k+1$, e volte para o passo 3 .

\subsubsection{Dificuldades Computacionais}

Embora a região factível do problema de barreira modificada seja relaxada, a seleção de um ponto inicial interior à essa região ainda pode ser trabalhosa para alguns problemas. O cálculo do tamanho do passo de atualização das variáveis pode ser uma dificuldade também, pois, caso isso seja feito sem um critério bem fundamentado, o processo computacional pode consumir muito tempo e até divergir. 


\section{Capítulo 4}

\section{Método da Função Lagrangiana \\ Barreira Modificada-Penalidade}

Métodos Lagrangianos são bastante eficazes quando aplicados à resolução de problemas de PNL com somente restrições de igualdade. Porém, na existência de restrições de desigualdade, exige-se que o conjunto das restrições ativas na solução seja conhecido, o que pode dificultar o processo de otimização.

Nos métodos de barreira, problemas de PNL com somente restrições de desigualdade são transformados em um problema de barreira cuja função auxiliar cresce indefinidamente na fronteira da região factível, restringindo a busca da solução do problema ao interior dessa região. No entanto, essa característica se torna uma deficiência à medida que o processo computacional de determinação da solução do problema de barreira se aproxima da fronteira da região factível: a matriz Hessiana da função auxiliar pode se tornar mal condicionada, exigindo métodos específicos para ser tratada, e a função barreira e suas derivadas de primeira e segunda ordem não são definidas na fronteira da região factível do problema.

Nos métodos de barreira modificada, essas dificuldades são superadas. Problemas de PNL com somente restrições de desigualdade são transformados em um problema de barreira modificada cuja região factível corresponde à do problema original relaxada. 
Nesses métodos, a matriz Hessiana da função barreira modificada permanece bem condicionada durante o processo computacional de determinação da solução do problema e as funções barreira modificada e suas derivadas de primeira e segunda ordem são definidas na fronteira da região factível do problema original.

No intuito de aproveitar as melhores qualidades desses métodos, Sousa (2006) propôs uma nova abordagem para resolução de problemas de PNL restritos, denominada método da Função Lagrangiana Barreira Modificada (FLBM), baseada na associação dos métodos primal-dual barreira logarítmica (FIACCO; McCORMICK, 1968) e de barreira modificada (POLYAK, 1992). Nela, as restrições de desigualdade são transformadas em restrições de igualdade a partir do acréscimo de variáveis de folga não negativas, cuja condição de não negatividade é tratada pela função barreira modificada de Frisch (POLYAK, 1992). Ao problema modificado, associa-se, então, uma função Lagrangiana. A solução do problema Lagrangiano é determinada pelo método de Newton-Lagrangiano, o qual fornece as direções de busca para a atualização das variáveis primais e duais associadas às restrições de igualdade. Os multiplicadores de Lagrange associados à condição de não negatividade das variáveis de folga são atualizados pela regra de Polyak (1992).

Apesar de ser definida em uma região factível relaxada do problema original, a função barreira modificada de Frisch não é definida em pontos exteriores a essa região. Isso exige que o processo de atualização das variáveis durante a determinação do ponto estacionário da FLBM seja monitorado a cada iteração. Por esse motivo, a abordagem de resolução de problemas de PNL desenvolvida neste capítulo propõe a substituição da função barreira modificada por uma FBMEQ no método desenvolvido por Sousa (2006). Dessa forma, os termos de barreira passam a ser definidos tanto no interior quanto no exterior da região factível relaxada do problema.

Essa nova abordagem para resolução de problemas de PNL restritos, denominada método da FLBMP, é baseada na associação dos métodos primal-dual barreira logarítmica (FIACCO; McCORMICK, 1968) e de barreira modificada-penalidade (BREITFELD; SHANNO, 1994; BREITFELD; SHANNO, 1996). Nela, as restrições de desigualdade são transformadas 
em restrições de igualdade a partir do acréscimo de variáveis de folga não negativas, cuja condição de não negatividade é tratada por uma FBMEQ (BREITFELD; SHANNO, 1994). Ao problema modificado, associa-se, então, uma função Lagrangiana. A solução do problema Lagrangiano é determinada pelo método de Newton-Lagrangiano. Os multiplicadores de Lagrange associados à condição de não negatividade das variáveis de folga são atualizados de acordo com o esquema proposto por Breitfeld e Shanno (1994).

Nas próximas seções são apresentados a FLBMP, seu método, seu algoritmo, e um exemplo ilustrativo.

\subsection{Definição da FLBMP}

Considere o seguinte problema de PNL:

$$
\begin{array}{lll}
\min & f(x) & \\
\text { s.a: } & g_{i}(x)=0 & i=1, \cdots, p \\
& h_{i}(x) \leq 0 & i=1, \cdots, q
\end{array}
$$

onde $x \in \mathbb{R}^{n} ; f: \mathbb{R}^{n} \rightarrow \mathbb{R} ; g: \mathbb{R}^{n} \rightarrow \mathbb{R}^{p}$, com $p<n$; e $h: \mathbb{R}^{n} \rightarrow \mathbb{R}^{q}$. Considere também que as funções $f, g$ e $h$ sejam de classe $C^{2}$.

Transformando as restrições de desigualdade em restrições de igualdade pelo acréscimo de variáveis de folga $s$ não negativas, (4.1) pode ser reescrito como:

$$
\begin{array}{lll}
\min & f(x) & \\
\text { s.a: } & g_{i}(x)=0 & i=1, \cdots, p \\
& h_{i}(x)+s_{i}=0 & i=1, \cdots, q \\
& s_{i} \geq 0 & i=1, \cdots, q
\end{array}
$$

onde $s \in \mathbb{R}^{q}$. 
A condição de não negatividade das variáveis de folga $s$ é tratada pela função barreira de Frisch modificada (POLYAK, 1992). Dessa forma, o problema (4.2) é transformado no seguinte problema:

$$
\begin{array}{lll}
\min & f(x)-\mu \sum_{i=1}^{q} \sigma_{i} \ln \left(\mu^{-1} s_{i}+1\right) & \\
\text { s.a: } & g_{i}(x)=0 & i=1, \cdots, p \\
& h_{i}(x)+s_{i}=0 & i=1, \cdots, q
\end{array}
$$

onde $\mu>0$ é o parâmetro de barreira modificada e $\sigma \in \mathbb{R}^{q}$ é o vetor das estimativas dos multiplicadores de Lagrange associados às condições de não negatividade das variáveis de folga $s, \operatorname{com} \sigma_{i} \geq 0$ para $i=1, \cdots, q$.

Como a função barreira modificada (4.3a) só é definida para $s>-\mu$, Breitfeld e Shanno (1996) propuseram a extrapolação quadrática dos termos logarítmicos em (4.3a), resultando na FBMEQ. Nela, os termos de barreira são definidos pela função $\phi: \mathbb{R} \rightarrow \mathbb{R}$, contínua e suave em $y \in \mathbb{R}$, da seguinte forma:

$$
\phi(y)= \begin{cases}\ln \left(\mu^{-1} y+1\right) & \text { se } \quad y \geq-\beta \mu \\ \frac{1}{2} q_{a} y^{2}+q_{b} y+q_{c} & \text { se } \quad y<-\beta \mu\end{cases}
$$

onde $\beta$ é um parâmetro definido no intervalo $(0 ; 1)$ e representa a tolerância da aproximação de $y$ à singularidade da função logarítmica. De acordo com Chen e Vassiliadis (2003), esse parâmetro pode ser constante e igual a 0,9 .

Como a função $\phi$ é contínua em $y \in \mathbb{R}$, a FBMEQ é, portanto, definida tanto no interior quanto na fronteira e no exterior da região factível relaxada.

Os coeficientes $q_{a}, q_{b}$ e $q_{c}$ em (4.4) são determinados de forma que os valores do termo da extrapolação quadrática de $\phi$ e suas derivadas de primeira e segunda ordem sejam iguais aos do termo logarítmico de $\phi$ e suas derivadas em $y=-\beta \mu$ (BREITFELD; 
SHANNO, 1994). Logo, as seguintes igualdades devem ser satisfeitas:

$$
\begin{aligned}
& \frac{1}{2} q_{a} y^{2}+q_{b} y+q_{c}=\ln \left(\frac{y}{\mu}+1\right) \\
& q_{a} y+q_{b}=\frac{1}{y+\mu} \\
& q_{a}=-\frac{1}{(y+\mu)^{2}}
\end{aligned}
$$

Portanto, substituindo $y=-\beta \mu$ em (4.7), obtém-se:

$$
q_{a}=-\frac{1}{[\mu(1-\beta)]^{2}}
$$

Substituindo $y=-\beta \mu$ e (4.8) em (4.6), obtém-se:

$$
q_{b}=\frac{1-2 \beta}{\mu(1-\beta)^{2}}
$$

E, finalmente, substituindo $y=-\beta \mu$, (4.8) e (4.9) em (4.5), obtém-se:

$$
q_{c}=\frac{\beta(2-3 \beta)}{2(1-\beta)^{2}}+\ln (1-\beta)
$$

Por fim, as restrições (4.3b) são incorporadas à função objetivo por multiplicadores de Lagrange $\lambda \in \mathbb{R}^{p}$, com $\lambda$ irrestrito, e as restrições (4.3c) são incorporadas à função objetivo por multiplicadores de Lagrange $\pi \in \mathbb{R}^{q}, \operatorname{com} \pi_{i} \geq 0$ na solução para $i=1, \cdots, q$.

Dessa forma, a FLBMP associada ao problema (4.1) é dada por:

$$
\mathcal{L}(x, s, \lambda, \pi, \sigma)=f(x)-\mu \sum_{i=1}^{q} \sigma_{i} \phi\left(s_{i}\right)+\sum_{i=1}^{p} \lambda_{i} g_{i}(x)+\sum_{j=1}^{q} \pi_{i}\left[h_{i}(x)+s_{i}\right]
$$




\subsection{Método da FLBMP}

No método da FLBMP, a solução do problema (4.1) é encontrada por meio da resolução de uma sequência de problemas de barreira modificada-penalidade, até que as condições de KKT sejam satisfeitas. Aplicando-se as condições necessárias de otimalidade à FLBMP, obtém-se o seguinte sistema de equações não lineares:

$$
\nabla \mathcal{L}(x, s, \lambda, \pi, \sigma)=0
$$

onde $\nabla \mathcal{L}$ é o gradiente da FLBMP em relação às variáveis $x, s, \lambda$ e $\pi$ :

$$
\nabla \mathcal{L}=\left[\begin{array}{l}
\nabla_{x} f(x)+J_{g}(x)^{T} \lambda+J_{h}(x)^{T} \pi \\
-\mu \sigma_{i} \phi^{\prime}\left(s_{i}\right)+\pi_{i} \\
g_{i}(x) \\
h_{i}(x)+s_{i}
\end{array}\right]
$$

sendo $J_{g}(x)=\left(\nabla_{x} g_{1}(x), \cdots, \nabla_{x} g_{p}(x)\right)^{T}$ a matriz Jacobiana das restrições de igualdade, $\nabla_{x} J_{h}(x)=\left(\nabla_{x} h_{1}(x), \cdots, \nabla_{x} h_{q}(x)\right)^{T}$ a matriz Jacobiana das restrições de desigualdade, e:

$$
\phi^{\prime}(y)= \begin{cases}(y+\mu)^{-1} & \text { se } \quad y \geq-\beta \mu \\ q_{a} y+q_{b} & \text { se } \quad y<-\beta \mu\end{cases}
$$

A solução do sistema de equações não lineares (4.12) é determinada pelo método de Newton, que, por sua vez, gera as direções de busca $\Delta d=(\Delta x, \Delta s, \Delta \lambda, \Delta \pi)$ através de sucessivas resoluções de um sistema de equações lineares representado, em sua forma simplificada, por:

$$
W \Delta d=-\nabla \mathcal{L}
$$


onde $W$ é a matriz Hessiana da FLBMP, dada por:

$$
W=\left[\begin{array}{cccc}
\nabla_{x x}^{2} \mathcal{L} & 0 & J_{g}(x)^{T} & J_{h}(x)^{T} \\
0 & -\mu \sigma S & 0 & I \\
J_{g}(x) & 0 & 0 & 0 \\
J_{h}(x) & I & 0 & 0
\end{array}\right]
$$

cujas submatrizes são:

$$
\nabla_{x x}^{2} \mathcal{L}=\nabla_{x x}^{2} f(x)+\sum_{i=1}^{p} \lambda_{i} \nabla_{x x}^{2} g_{i}(x)+\sum_{i=1}^{q} \pi_{i} \nabla_{x x}^{2} h_{i}(x)
$$

e a submatriz $S$ é uma matriz diagonal definida em função de $s$ da seguinte forma:

$$
S=\left[\begin{array}{ccc}
\phi^{\prime \prime}\left(s_{1}\right) & & 0 \\
& \ddots & \\
0 & & \phi^{\prime \prime}\left(s_{q}\right)
\end{array}\right]
$$

com:

$$
\phi^{\prime \prime}(y)= \begin{cases}-(y+\mu)^{-2} & \text { se } \quad y \geq-\beta \mu \\ q_{a} & \text { se } \quad y<-\beta \mu\end{cases}
$$

As variáveis $x, s, \lambda$ e $\pi$ são atualizadas por:

$$
\begin{aligned}
& x^{k+1}=x^{k}+\alpha_{p} \Delta x^{k} \\
& s^{k+1}=s^{k}+\alpha_{p} \Delta s^{k} \\
& \lambda^{k+1}=\lambda^{k}+\alpha_{d} \Delta \lambda^{k} \\
& \pi^{k+1}=\pi^{k}+\alpha_{d} \Delta \pi^{k}
\end{aligned}
$$


onde $\alpha_{p}$ e $\alpha_{d}$ são os passos primal e dual utilizados na atualização das variáveis. O passo primal é determinado através de um processo de busca linear satisfazendo, por exemplo, a condição de Armijo (CHEN; VASSILIADIS, 2003). O passo dual é calculado de forma que cada multiplicador $\pi$ permaneça maior ou igual a zero, isto é (SOUSA, 2006):

$$
\alpha_{d}=\min \left\{\left(\min _{\Delta \pi<0 \mathrm{e} \pi>0} \frac{\pi}{|\Delta \pi|}\right), 1\right\}
$$

Os multiplicadores de Lagrange associados à condição de não negatividade das variáveis de folga $s$ são atualizados de acordo com o esquema proposto por Breitfeld e Shanno (1994), da seguinte forma:

$$
\sigma_{i}^{k+1}= \begin{cases}\mu \sigma_{i}^{k}\left(\mu^{-1} s_{i}^{k}+1\right)^{-1} & \text { se } \quad \sigma_{i}^{k} \geq-\beta \mu \\ \mu \sigma_{i}^{k}\left(q_{a} s_{i}^{k}+q_{b}\right) & \text { se } \quad \sigma_{i}^{k}<\beta \mu\end{cases}
$$

para $i=1, \cdots, q$. Esses multiplicadores são inicializados por $\sigma_{i}^{0}=1$ (SOUSA, 2006).

Por fim, o parâmetro de barreira $\mu$ é atualizado por uma simples regra de redução:

$$
\mu^{k+1}=\frac{\mu^{k}}{\tau}
$$

onde $\tau>1$ e geralmente $\tau=2$ ou $\tau=10$. Como a FBMEQ é definida para qualquer valor de $\mu$, esse parâmetro é inicializado por um valor positivo arbitrário, geralmente igual a $\mu^{0}=10^{-1}$ ou $\mu^{0}=10^{-2}$ (BREITFELD; SHANNO, 1994).

O método converge quando as seguintes condições forem satisfeitas (TORRES; QUINTANA, 1998):

$$
\begin{array}{lll}
v_{1}^{k} \leq \xi_{1} & & \\
v_{2}^{k} \leq \xi_{2} & \text { ou } & \\
v_{3}^{k} \leq \xi_{3} & & v_{2}^{k} \leq \xi_{2}
\end{array}
$$


onde:

$$
\begin{aligned}
v_{1}^{k} & =\|\mathcal{L}(x, s, \lambda, \pi, \sigma)\|_{\infty} \\
v_{2}^{k} & =\frac{\left|f\left(x^{k}\right)-f\left(x^{k-1}\right)\right|}{1+\left|f\left(x^{k}\right)\right|} \\
v_{3}^{k} & =\max \left\{h_{i}\left(x^{k}\right)\right\} \quad \text { para } i=1, \cdots, q
\end{aligned}
$$

Se os critérios $v_{1}^{k} \leq \xi_{1}, v_{2}^{k} \leq \xi_{2}$ e $v_{3}^{k} \leq \xi_{3}$ forem satisfeitos, então a factibilidade primal e dual escalar estão garantidas, o que significa que na iteração $k$, tem-se uma solução que satisfaz as condições de KKT com precisão $\xi_{1}$. Quando problemas numéricos impedem o alcance destas, o método para assim que a factibilidade das restrições de igualdade for alcançada e as mudanças no valor da função objetivo forem desprezíveis. Para problemas de grande porte, Sousa (2006) adotou os seguintes valores para essas tolerâncias: $\xi_{1}=10^{-3}$, $\xi_{2}=10^{-2}$ e $\xi_{3}=10^{-1}$. Além disso, as condições $s_{i} \geq 0$ e $\pi_{i} \geq 0$ para $i=1, \cdots, q$ devem ser satisfeitas na solução.

\subsection{Algoritmo}

1) Dado o problema (4.1) construa a FLBMP (4.11);

2) Faça $k=0$;

Dê um valor inicial para as variáveis $x^{0}, s^{0}, \lambda^{0}$ e $\pi^{0}$, tal que $\pi_{i}^{0} \geq 0$ para $i=1, \cdots, q$, e para o parâmetro $\mu^{0}$;

Dê uma estimativa para os multiplicadores de Lagrange $\sigma_{i}^{0} \geq 0$, para $i=1, \cdots, q$; Especifique um valor para o fator $\tau>1$ e as tolerâncias $\xi_{1}, \xi_{2}$ e $\xi_{3}$;

3) Determine o gradiente (4.13) e a matriz Hessiana da FLBMP(4.16), e resolva o sistema (4.15); 
4) Atualize as variáveis do problema por (4.19), determinando $x^{k+1}, s^{k+1}, \lambda^{k+1}$ e $\pi^{k+1}$ e vá para o passo 5 ;

5) Se o critério de parada do método de Newton for satisfeito, vá para o passo 6;

Caso contrário, volte para o passo 3 ;

6) Se as condições (4.23) forem satisfeitas para $x^{k+1}, s^{k+1}, \lambda^{k+1}, \pi^{k+1}$ e $\sigma^{k}$, e se $s^{k+1} \geq 0$ e $\pi^{k+1} \geq 0$, pare.

Caso contrário, atualize os multiplicadores de Lagrange $\sigma$ por (4.21), determinando $\sigma^{k+1}$, e o parâmetro $\mu$ por $(4.22)$, faça $k=k+1$, e volte para o passo 3 .

\subsection{Exemplo Numérico}

Para exemplificar o uso do método da FLBMP, considere o seguinte problema:

$$
\begin{array}{ll}
\min & \left(x_{1}-2\right)^{4}+\left(x_{1}-2 x_{2}\right)^{2} \\
\text { s.a: } & x_{1}+x_{2}=3 \\
& x_{1}^{2}-x_{2} \leq 0 \\
& 1,5 \leq x_{2} \leq 2
\end{array}
$$

Transformando as restrições de desigualdade em igualdades através da inserção de variáveis de folga $s$ não negativas, (4.27) pode ser reescrito na seguinte forma:

$$
\begin{aligned}
& \min \left(x_{1}-2\right)^{4}+\left(x_{1}-2 x_{2}\right)^{2} \\
& \text { s.a: } \quad x_{1}+x_{2}-3=0 \\
& x_{1}^{2}-x_{2}+s_{1}=0 \\
& 1,5-x_{2}+s_{2}=0 \\
& x_{2}-2+s_{3}=0 \\
& s_{i} \geq 0 \quad i=1, \cdots, 3
\end{aligned}
$$


A FLBMP associada ao problema (4.28) é dada por:

$$
\begin{aligned}
\mathcal{L}(x, s, \lambda, \pi)= & \left(x_{1}-2\right)^{4}+\left(x_{1}-2 x_{2}\right)^{2}-\mu\left[\sigma_{1} \phi\left(s_{1}\right)+\sigma_{2} \phi\left(s_{2}\right)+\sigma_{3} \phi\left(s_{3}\right)\right]+ \\
& \lambda_{1}\left(x_{1}+x_{2}-3\right)+\pi_{1}\left(x_{1}^{2}-x_{2}+s_{1}\right)+\pi_{2}\left(1,5-x_{2}+s_{2}\right)+ \\
& \pi_{3}\left(x_{2}-2+s_{3}\right)
\end{aligned}
$$

onde:

$$
\phi\left(s_{i}\right)=\left\{\begin{array}{lll}
\ln \left(\mu^{-1} s_{i}+1\right) & \text { se } & s_{i} \geq-\beta \mu \\
\frac{1}{2} q_{a} s_{i}^{2}+q_{b} s_{i}+q_{c} & \text { se } & s_{i}<-\beta \mu
\end{array}\right.
$$

para $i=1, \cdots, 3$, e os coeficientes $q_{a}, q_{b}$ e $q_{c}$ da extrapolação quadrática da função $\phi$ são calculados, respectivamente, por (4.8), (4.9) e (4.10).

Aplicando-se as condições necessárias de otimalidade à FLBMP (4.29), obtém-se o seguinte sistema de equações não lineares:

$$
\nabla \mathcal{L}\left(x_{1}, x_{2}, s_{1}, s_{2}, s_{3}, \lambda, \pi_{1}, \pi_{2}, \pi_{3}\right)=0
$$

onde:

$$
\nabla \mathcal{L}=\left[\begin{array}{l}
4\left(x_{1}-2\right)^{3}+2 x_{1}-4 x_{2}+\lambda+2 \pi_{1} x_{1} \\
-4 x_{1}+8 x_{2}+\lambda-\pi_{1}-\pi_{2}+\pi_{3} \\
-\mu \sigma_{1} \phi^{\prime}\left(s_{1}\right)+\pi_{1} \\
-\mu \sigma_{2} \phi^{\prime}\left(s_{2}\right)+\pi_{2} \\
-\mu \sigma_{3} \phi^{\prime}\left(s_{3}\right)+\pi_{3} \\
x_{1}+x_{2}-3 \\
x_{1}^{2}-x_{2}+s_{1} \\
1,5-x_{2}+s_{2} \\
x_{2}-2+s_{3}
\end{array}\right]
$$


A solução de (4.31) é determinada pelo método de Newton. Portanto, tem-se

$$
W \Delta d=-\nabla \mathcal{L}
$$

onde $\Delta d=(\Delta x, \Delta s, \Delta \lambda, \Delta \pi)^{T}$, e $W$ é a matriz Hessiana da FLBMP (4.29), dada por:

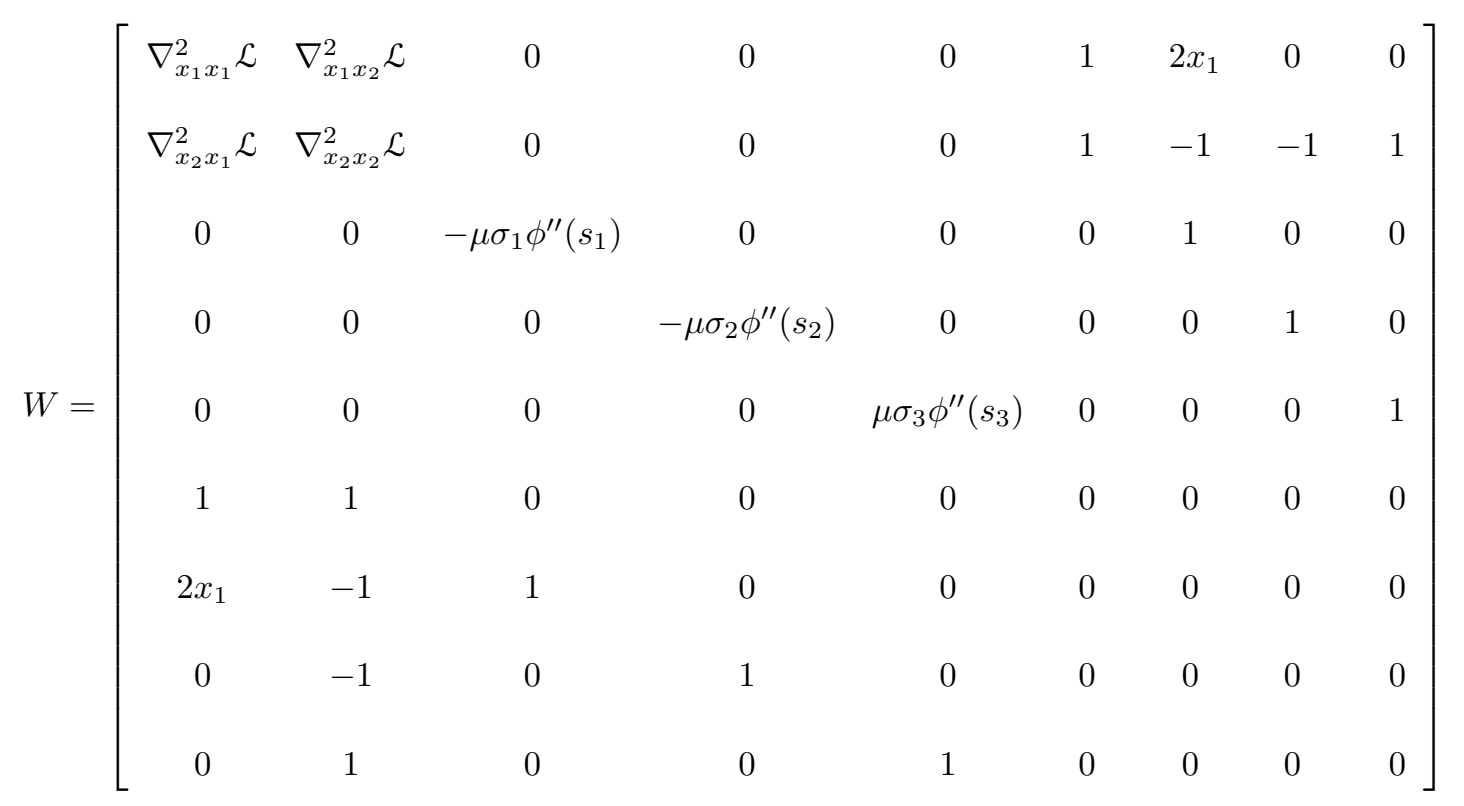

sendo:

$$
\begin{aligned}
& \nabla_{x_{1} x_{1}}^{2} \mathcal{L}=12\left(x_{1}-2\right)^{2}+2+2 \pi_{1} \\
& \nabla_{x_{1} x_{2}}^{2} \mathcal{L}=\nabla_{x_{2} x_{1}}^{2} \mathcal{L}=-4 \\
& \nabla_{x_{2} x_{2}}^{2} \mathcal{L}=8
\end{aligned}
$$

Para resolver o problema (4.27), o método da FLBMP foi inicializado com dois pontos iniciais distintos. A escolha desses pontos foi feita de forma a se explorar algumas características do método da FLBMP. Nesses testes, considerou-se $\mu^{0}=10^{-1}$ e as tolerâncias para convergência foram $\xi_{1}=10^{-5}, \xi_{2}=10^{-4}$ e $\xi_{3}=10^{-3}$. 
Tabela 4.1 - Convergência do método da FLBMP aplicado ao problema (4.27), para $x_{1}^{0}=1,1$ e $x_{2}^{0}=1,7$ - Função objetivo, variáveis primais e parâmetro $\mu$.

\begin{tabular}{cccccccc}
\hline Iteração & F. Obj. & $x_{1}$ & $x_{2}$ & $s_{1}$ & $s_{2}$ & $s_{3}$ & $\mu$ \\
\hline 0 & 5,9461 & 1,1000 & 1,7000 & 0,4900 & 0,2000 & 0,3000 & $10^{-1}$ \\
1 & 4,3377 & 1,3228 & 1,6772 & $-0,0726$ & 0,1772 & 0,3228 & $10^{-1}$ \\
2 & 4,5766 & 1,3053 & 1,6947 & $-0,0091$ & 0,1947 & 0,3053 & $10^{-2}$ \\
3 & 4,6114 & 1,3028 & 1,6972 & $-0,0000$ & 0,1972 & 0,3028 & $10^{-3}$ \\
4 & 4,6072 & 1,3031 & 1,6969 & $-0,0011$ & 0,1969 & 0,3031 & $10^{-4}$ \\
5 & 4,6114 & 1,3028 & 1,6972 & $-0,0000$ & 0,1972 & 0,3028 & $10^{-5}$ \\
\hline
\end{tabular}

Tabela 4.2 - Convergência do método da FLBMP aplicado ao problema (4.27), para $x_{1}^{0}=1,1$ e $x_{2}^{0}=1,7$ - Variáveis duais.

\begin{tabular}{cccccccc}
\hline Iteração & $\lambda$ & $\pi_{1}$ & $\pi_{2}$ & $\pi_{3}$ & $\sigma_{1}$ & $\sigma_{2}$ & $\sigma_{3}$ \\
\hline 0 & 0,0000 & 1,0000 & 1,0000 & 1,0000 & 1,0000 & 1,0000 & 1,0000 \\
1 & $-4,3518$ & 3,6503 & 0,3608 & 0,2365 & 1,0000 & 1,0000 & 1,0000 \\
2 & $-4,5010$ & 3,8346 & 0,0018 & 0,0008 & 0,3650 & 0,0361 & 0,0237 \\
3 & $-4,5099$ & 3,8568 & 0,0000 & 0,0000 & 3,8346 & 0,0000 & 0,0000 \\
4 & $-4,5089$ & 3,8542 & 0,0000 & 0,0000 & 0,0039 & 0,0000 & 0,0000 \\
5 & $-4,5099$ & 3,8568 & 0,0000 & 0,0000 & 3,8542 & 0,0000 & 0,0000 \\
\hline
\end{tabular}

Teste 1:

As Tabelas 4.1 e 4.2 apresentam o processo de convergência do método da FLBMP para um ponto inicial no interior da região definida pelas restrições de desigualdade: $x_{1}=1,1$ e $x_{2}=1,7$. As variáveis $s$ são inicializadas, respectivamente, a partir das restrições $(4.28 \mathrm{c}),(4.28 \mathrm{~d})$ e $(4.28 \mathrm{e})$ do problema modificado. Os multiplicadores de Lagrange $\lambda, \pi$ e $\sigma$ foram inicializados, respectivamente, por 0,1 e 1 .

Como pode ser visto, o método da FLBMP convergiu em 5 iterações, com a restrição de desigualdade $(4.27 \mathrm{c})$ ativa. 


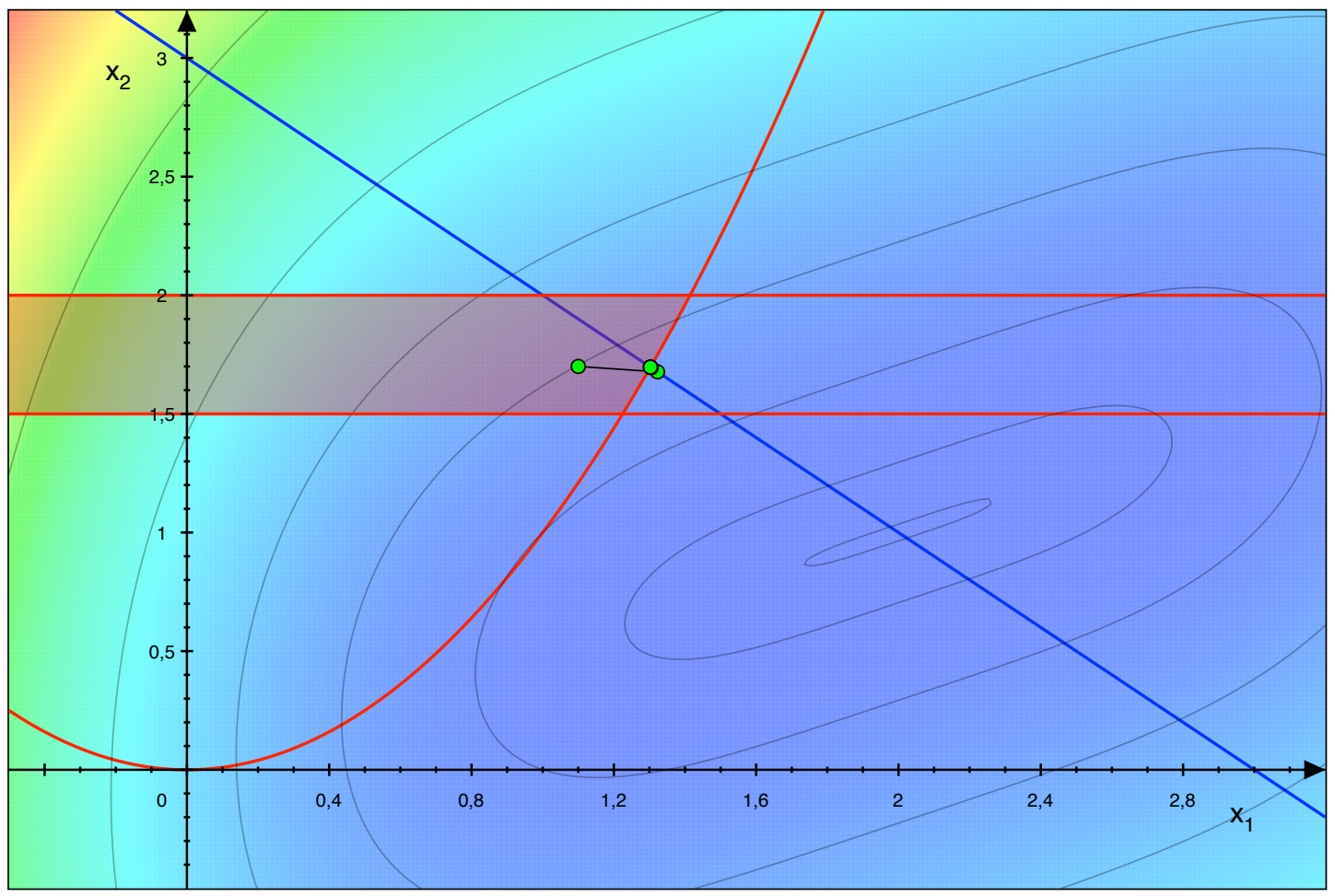

Figura 4.1 - Convergência do método da FLBMP aplicado ao problema (4.27), para $x_{1}^{0}=1,1$ e $x_{2}^{0}=1,7$.

O processo de convergência do método da FLBMP mostrado nas Tabelas 4.1 e 4.2 é representado graficamente na Figura 4.1. Nesta figura, a restrição de igualdade (4.27b) é representada na cor azul, e as restrições de desigualdade (4.27c) e canalizada $(4.27 \mathrm{~d})$ são representadas na cor vermelha. A região definida pelas restrições de desigualdade do problema é representada na cor vermelha suave.

Através da Figura 4.1, pode-se ver que, neste caso, o método converge a partir da região infactível do problema (4.27).

\section{Teste 2:}

No segundo teste, o método da FLBMP foi inicializado com um ponto infactível: $x_{1}=1,5$ e $x_{2}=1,0 ;$ as variáveis de folga $s$ e os multiplicadores de Lagrange $\lambda$ e $\pi$ foram inicializados da mesma forma que no teste anterior. Os multiplicadores de Lagrange $\sigma$ 
Tabela 4.3 - Convergência do método da FLBMP aplicado ao problema (4.27), para $x_{1}^{0}=1,5$ e $x_{2}^{0}=1,0$ e multiplicadores $\sigma^{*}$ conhecidos - Função objetivo, variáveis primais e parâmetro $\mu$.

\begin{tabular}{cccccccc}
\hline Iteração & F. Obj. & $x_{1}$ & $x_{2}$ & $s_{1}$ & $s_{2}$ & $s_{3}$ & $\mu$ \\
\hline 0 & 0,3125 & 1,5000 & 1,0000 & $-1,2500$ & $-0,5000$ & 1,0000 & $10^{-1}$ \\
1 & 4,6114 & 1,3028 & 1,6972 & $-0,0000$ & 0,1972 & 0,3028 & $10^{-1}$ \\
\hline
\end{tabular}

Tabela 4.4 - Convergência do método da FLBMP aplicado ao problema (4.27), para $x_{1}^{0}=1,5$ e $x_{2}^{0}=1,0$ e multiplicadores $\sigma^{*}$ conhecidos - Variáveis duais.

\begin{tabular}{cccccccc}
\hline Iteração & $\lambda$ & $\pi_{1}$ & $\pi_{2}$ & $\pi_{3}$ & $\sigma_{1}$ & $\sigma_{2}$ & $\sigma_{3}$ \\
\hline 0 & 0,0000 & 1,0000 & 1,0000 & 1,0000 & 3,8542 & 0,0000 & 0,0000 \\
1 & $-4,5099$ & 3,8568 & 0,0000 & 0,0000 & 3,8542 & 0,0000 & 0,0000 \\
\hline
\end{tabular}

foram inicializados com os valores ótimos obtidos no teste anterior e, dessa forma, espera-se que o algoritmo convirja em 1 iteração, independentemente do valor inicial de $\mu$.

As Tabelas 4.3 e 4.4 apresentam o processo de convergência do método da FLBMP para este segundo teste. O resultado mostrado nas Tabelas 4.3 e 4.4 é representado graficamente na Figura 4.2.

Como esperado, o método da FLBMP convergiu em 1 iteração, mesmo quando inicializado com um ponto no exterior da região factível do problema. 


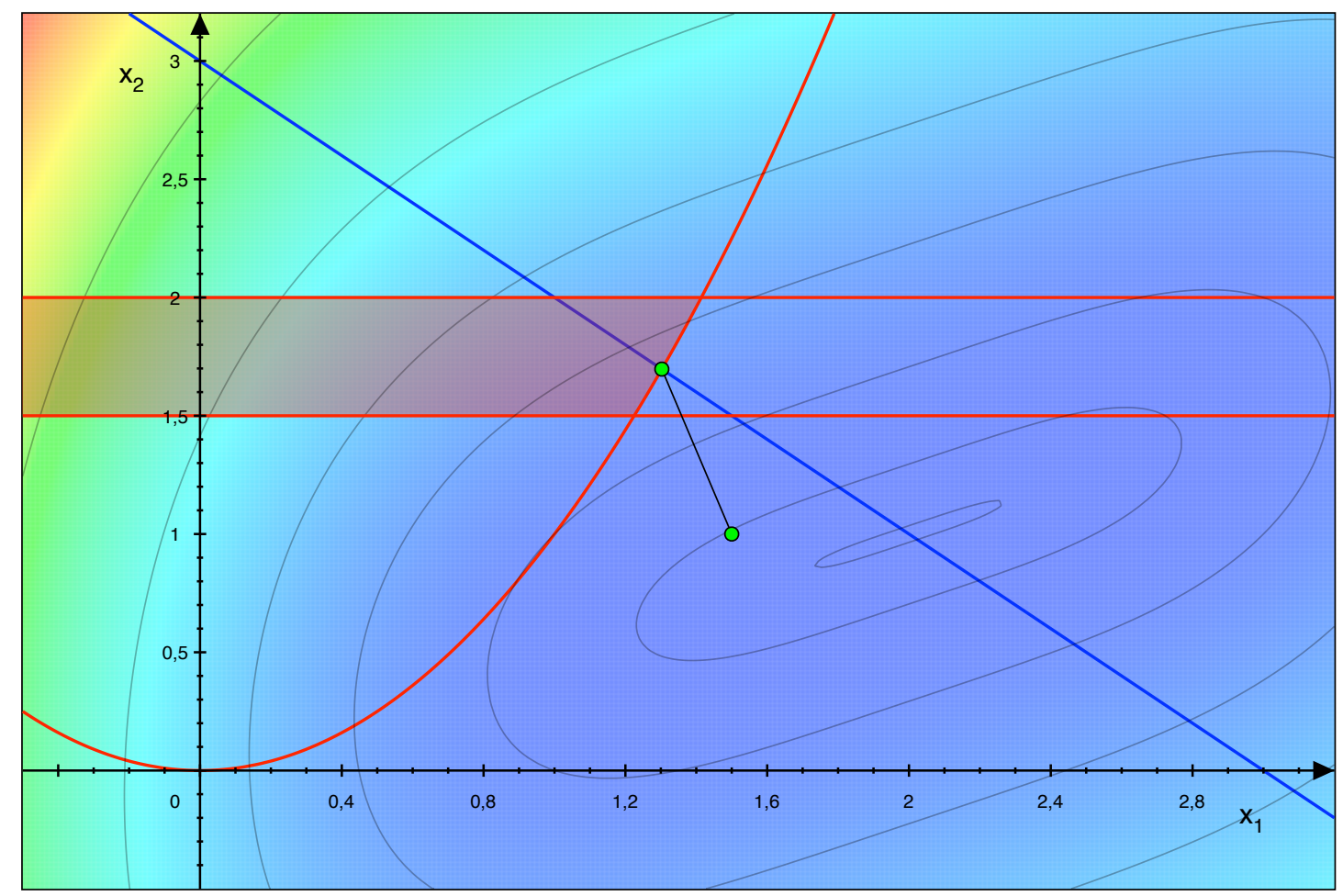

Figura 4.2 - Convergência do método da FLBMP aplicado ao problema (4.27), para $x_{1}^{0}=1,5$ e $x_{2}^{0}=1,0$ e multiplicadores $\sigma^{*}$ conhecidos. 


\section{Capítulo 5}

\section{Algoritmo da FLBMP-Discreto}

Neste capítulo, o método da FLBMP é aplicado à resolução de problemas de PNL com variáveis contínuas e discretas. Nessa abordagem, denominada algoritmo da FLBMP-discreto, as variáveis discretas são tratadas como contínuas por funções senoidais $\psi$ incorporadas na função objetivo do problema original. Essas funções se anulam quando essas variáveis assumirem valores discretos e sua amplitude é controlada por um parâmetro $\gamma>0$. Assim, tem-se um problema de PNL com somente variáveis contínuas. A solução do problema modificado equivale à do problema original (SOLER, 2011).

\subsection{Definição do Problema Modificado}

Considere o seguinte problema de PNL com variáveis contínuas e discretas:

$$
\begin{array}{lll}
\min & f(x) & \\
\text { s.a: } & g_{i}(x)=0 & i=1, \cdots, p \\
& h_{i}(x) \leq 0 & i=1, \cdots, q \\
& x_{1_{i}}^{\text {min }} \leq x_{1_{i}} \leq x_{1_{i}}^{\text {max }} & i=1, \cdots, m_{1} \\
& x_{2_{i}} \in \mathcal{D}_{x_{i}} & i=1, \cdots, m_{2}
\end{array}
$$


onde $x_{1} \in \mathbb{R}^{m_{1}}$ é o vetor das variáveis contínuas e $x_{2} \in \mathcal{D}_{x} \subset \mathbb{R}^{m_{2}}$ é o vetor das variáveis discretas, com $x=\left(x_{1}, x_{2}\right) ; \mathcal{D}_{x}$ é o conjunto dos valores discretos de cada variável $x_{2}$; $f: \mathbb{R}^{m_{1}} \times \mathbb{R}^{m_{2}} \rightarrow \mathbb{R} ; g: \mathbb{R}^{m_{1}} \times \mathbb{R}^{m_{2}} \rightarrow \mathbb{R}^{p}$, com $p<m_{1}+m_{2} ;$ e $h: \mathbb{R}^{m_{1}} \times \mathbb{R}^{m_{2}} \rightarrow \mathbb{R}^{q}$

De acordo com Soler (2011), considerando $y \in \mathbb{R}^{m_{2}}$, a função $\psi: \mathbb{R} \rightarrow \mathbb{R}$ para o tratamento de variáveis discretas $x_{2}$ na resolução de problemas como (5.1) é definida como:

$$
\psi\left(y_{i}\right)= \begin{cases}0 & \text { se } y_{i}<y_{i}^{\text {min }} \\ {\left[\operatorname{sen}\left(\frac{y_{i}}{y_{i}^{\text {sup }}-y_{i}^{\text {inf }}} \pi+\alpha\right)\right]^{2 \beta}} & \text { se } y_{i}^{\text {min }} \leq y_{i} \leq y_{i}^{\max } \\ 0 & \text { se } y_{i}>y_{i}^{\max }\end{cases}
$$

para $i=1, \cdots, m_{2}$ e onde:

- $\beta>0$ é uma constante inteira que determina a forma da função $\psi$;

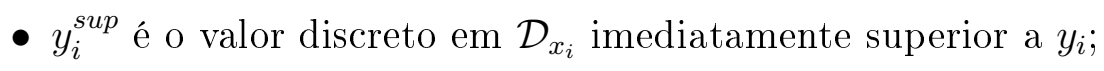

- $y_{i}^{\text {inf }}$ é o valor discreto em $\mathcal{D}_{x_{i}}$ imediatamente inferior a $y_{i}$;

- $y_{i}^{\text {min }}$ é o menor valor do conjunto de valores discretos $\mathcal{D}_{x_{i}}$;

- $y_{i}^{\max }$ é o maior valor do conjunto de valores discretos $\mathcal{D}_{x_{i}}$;

- $\alpha$ é uma constante definida no intervalo $[0 ; \pi]$ tal que a função $\psi$ se anule em $y_{i}=x_{2_{i}} \in \mathcal{D}_{x_{i}}$.

Isso significa que a função $\psi$ é definida da seguinte forma:

$$
\psi\left(y_{i}\right)= \begin{cases}0 & \text { se } y_{i} \in \mathcal{D}_{x_{i}} \\ \rho>0 & \text { c.c. }\end{cases}
$$

ou seja, $\psi$ se anula dentro do intervalo $\left[y_{i}^{\min } ; y_{i}^{\max }\right]$ somente se $y_{i}$ assumir valores discretos. 
Através da incorporação da função $\psi$ na função objetivo do problema (5.1), obtém-se o seguinte problema modificado:

$$
\begin{array}{lll}
\min & f(x)+\gamma \sum_{i=1}^{m_{2}} \psi\left(x_{2_{i}}\right) & \\
\text { s.a: } & g_{i}(x)=0 & i=1, \cdots, p \\
& h_{i}(x) \leq 0 & i=1, \cdots, q \\
x_{1_{i}}^{\text {min }} \leq x_{1_{i}} \leq x_{1_{i}}^{\text {max }} & i=1, \cdots, m_{1} \\
x_{2_{i}}^{\text {min }} \leq x_{2_{i}} \leq x_{2_{i}}^{\text {max }} & & i=1, \cdots, m_{2}
\end{array}
$$

onde $\gamma>0$ é o parâmetro de controle da amplitude da função $\psi ; x_{1} \in \mathbb{R}^{m_{1}}$ e $x_{2} \in \mathbb{R}^{m_{2}}$ são vetores de variáveis contínuas, com $x=\left(x_{1}, x_{2}\right)$ e $x \in \mathbb{R}^{n} ; f: \mathbb{R}^{n} \rightarrow \mathbb{R} ; g: \mathbb{R}^{n} \rightarrow \mathbb{R}^{p}$, com $p<n$; e $h: \mathbb{R}^{n} \rightarrow \mathbb{R}^{q}$. No problema modificado (5.3), as funções $f, g$ e $h$ são de classe $C^{2}$ e $x_{2}^{\min }, x_{2}^{\max } \in \mathbb{R}^{m_{2}}$ são os vetores dos limites inferior e superior das variáveis contínuas $x_{2}, \operatorname{com} x_{2_{i}}^{\min }=\min \left\{D_{x_{i}}\right\}$ e $x_{2_{i}}^{\max }=\max \left\{D_{x_{i}}\right\}$ para $i=1, \cdots, m_{2}$.

Uma solução ótima do problema modificado (5.3) equivale a uma solução do problema original (5.1) (SOLER, 2011).

A função objetivo (5.3a) é denominada função objetivo aumentada, pois esta consiste na função objetivo do problema original acrescida das funções $\psi$.

\subsection{Algoritmo da FLBMP-Discreto}

O algoritmo da FLBMP-discreto consiste na resolução de uma sequência de problemas modificados como (5.3) pelo método da FLBMP até que todas as variáveis associadas às variáveis discretas do problema original assumam valores discretos.

Nesse algoritmo, a determinação adequada de um valor para o parâmetro $\gamma$ é fundamental para que a solução do problema modificado (5.3) seja encontrada. Dessa forma, para que o valor de $\gamma$ seja determinado corretamente, Soler, Sousa e Costa (2012) propõem a resolução de uma série de problemas como (5.3) para diferentes valores de $\gamma$, 
com $\gamma$ crescendo gradualmente. A forma do ajuste do crescimento de $\gamma$ é a seguinte:

$$
\gamma^{k+1}=\tau \gamma^{k}
$$

onde $\tau$ é o fator de ajuste do crescimento de $\gamma$, definido no intervalo $(1 ; 10]$.

O processo de resolução do problema (5.3) e ajuste do parâmetro $\gamma$ continua até que a seguinte condição seja satisfeita para todas variáveis $x_{2}$ do problema modificado:

$$
\left|x_{2_{i}}-x_{2_{i}}^{\prime}\right|<\xi
$$

para $i=1, \cdots, m_{2}$ e onde $x_{2_{i}}^{\prime} \in \mathcal{D}_{x_{i}}$ é o valor discreto mais próximo da variável $x_{2_{i}}$, e $\xi$ é a tolerância para convergência do algoritmo da FLBMP-discreto.

\subsubsection{Influência dos Parâmetros $\gamma$ e $\beta$ na Função $\psi$}

Para a análise da influência dos parâmetros $\gamma$ e $\beta$ na função $\psi(x)$, considere o seguinte problema unidimensional:

$$
\begin{array}{ll}
\min & x^{2}+1 \\
\text { s.a: } & x \in \mathcal{X}
\end{array}
$$

onde $\mathcal{X}=\{-2 ;-1 ; 0 ; 1 ; 2 ; 3\}$. A representação gráfica deste problema é dada na Figura 5.1.

Como os elementos do conjunto $\mathcal{X}$ são números inteiros e a distância entre dois valores discretos consecutivos de $\mathcal{X}$ é constante e igual a 1, pode-se escolher $\alpha=0$ na definição da função $\psi$, pois, dessa forma, essa função se anula para esses valores 


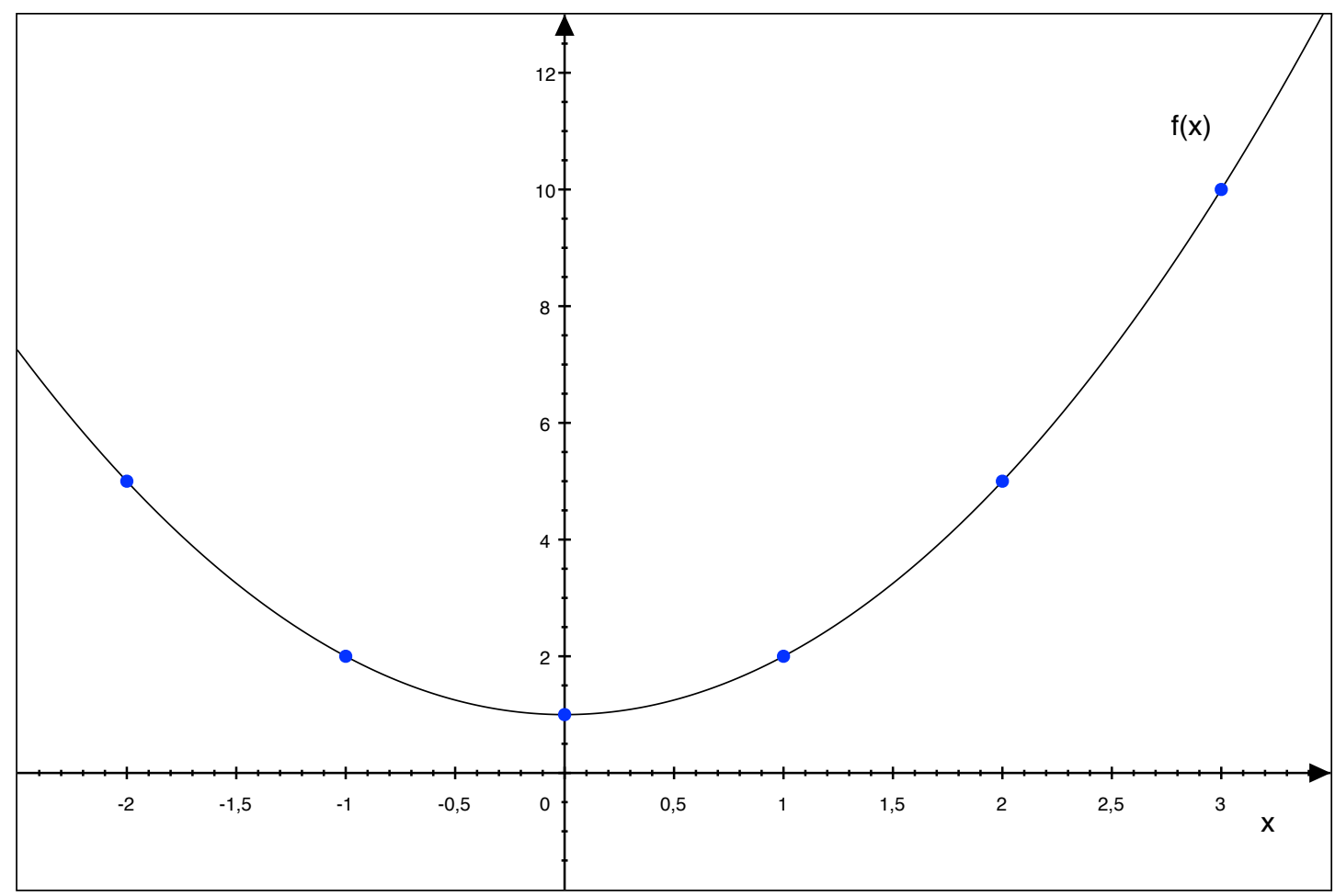

Figura 5.1 - Representação gráfica do problema (5.6), onde $x \in \mathcal{X}$.

pertencentes a $\mathcal{X}$. A função $\psi$ é dada por:

$$
\psi(x)=\left\{\begin{array}{lll}
0 & \text { se } \quad x<-2 \\
{[\operatorname{sen}(x \pi)]^{2 \beta}} & \text { se } \quad-2 \leq x \leq 3 \\
0 & \text { se } \quad x>3
\end{array}\right.
$$

O problema (5.6) é modificado da seguinte forma:

$$
\begin{array}{ll}
\min & x^{2}+1+\gamma[\operatorname{sen}(x \pi)]^{2 \beta} \\
\text { s.a: } & -2 \leq x \leq 3
\end{array}
$$

Através da introdução das modificações apresentadas acima, uma solução ótima do problema (5.8) equivale a uma solução do problema (5.6) (SOLER, 2011).

Para a análise da influência do parâmetro $\beta$ na função $\psi$, considere a representação gráfica da função (5.7) dada nas Figuras 5.2, 5.3 e 5.4, para $\beta=\{1 ; 2 ; 4\}$. Como pode ser 


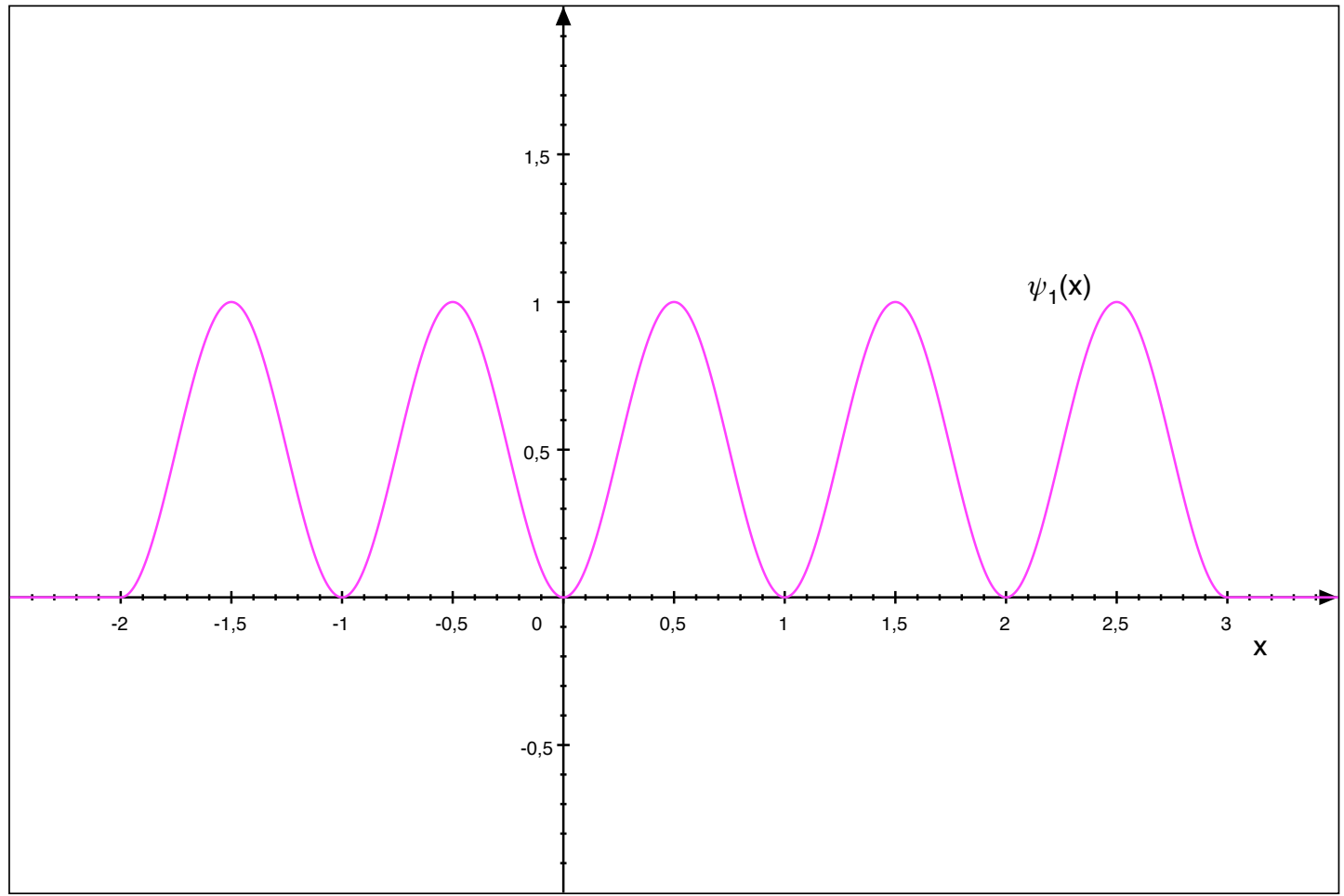

Figura 5.2 - Função $\psi$ definida por $x \in \mathcal{X}$, para $\beta=1$.

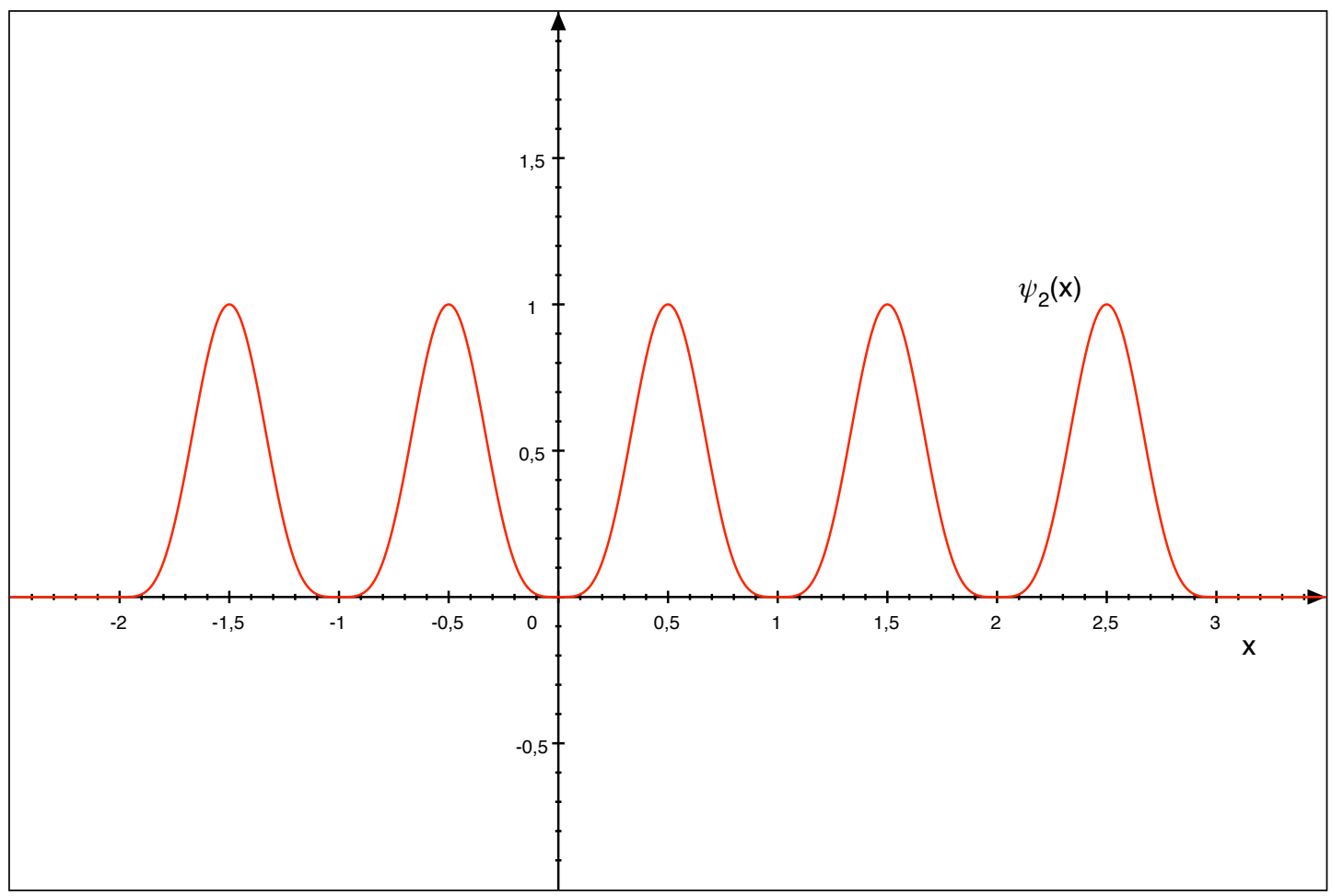

Figura 5.3 - Função $\psi$ definida por $x \in \mathcal{X}$, para $\beta=2$. 


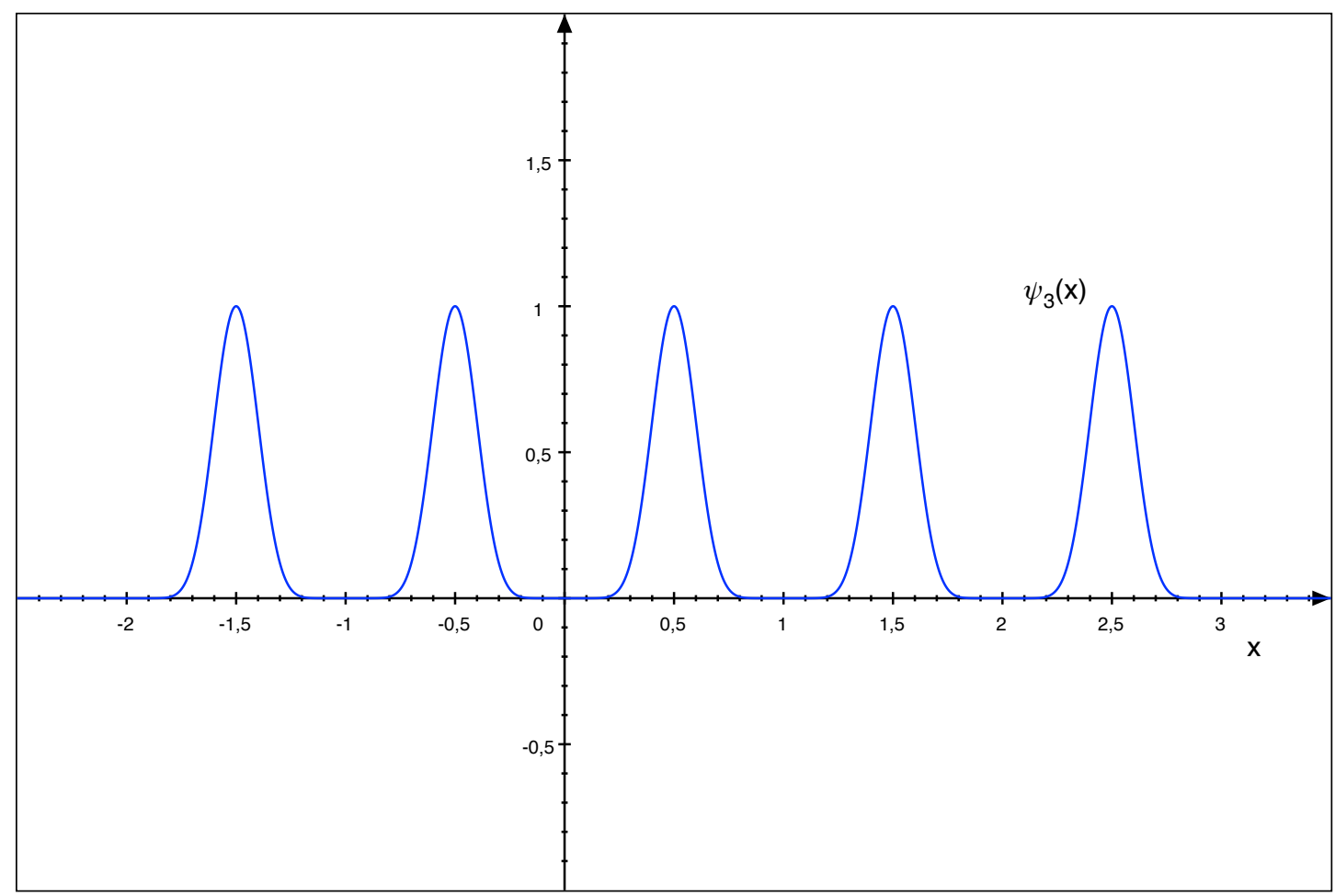

Figura 5.4 - Função $\psi$ definida por $x \in \mathcal{X}$, para $\beta=4$.

observado, os valores do parâmetro $\beta$ influenciam na forma da função $\psi$ : quanto maior for o valor desse parâmetro, mais "achatadas" e, portanto, menos penalizadas serão as regiões vizinhas aos valores definidos em $\mathcal{X}$.

Para a análise da influência do parâmetro $\gamma$ na função $\psi$, considere a representação gráfica da função objetivo aumentada (5.8a) dada nas Figuras 5.5, 5.6 e 5.7, para $\gamma=\{0,3 ; 1,3 ; 3,0\}$ e com $\beta=1$. Como pode ser observado, esse parâmetro influencia na amplitude da função $\psi$ e, consequentemente, na semelhança entre a função objetivo do problema original e a função objetivo aumentada. Para valores grandes de $\gamma$, a função objetivo aumentada perde as características da função objetivo original e, nesse caso, a solução do problema (5.8) pode não ser equivalente à do problema (5.6). Por outro lado, para valores muito pequenos de $\gamma$, a solução do problema (5.8) pode assumir um valor não discreto para a variável $x$. 


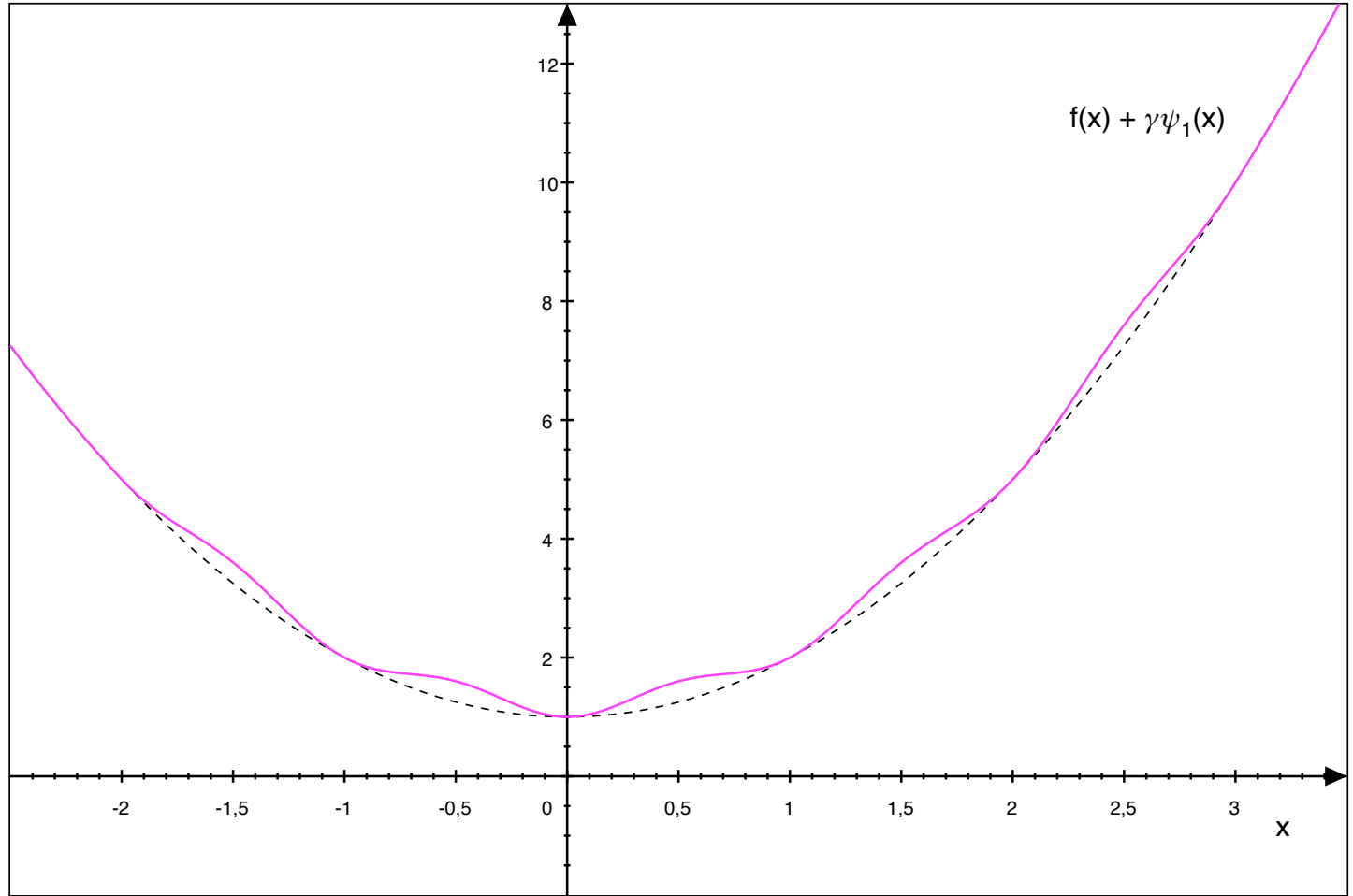

Figura 5.5 - Função objetivo aumentada definida por $x \in \mathcal{X}$, para $\gamma=0,3$.

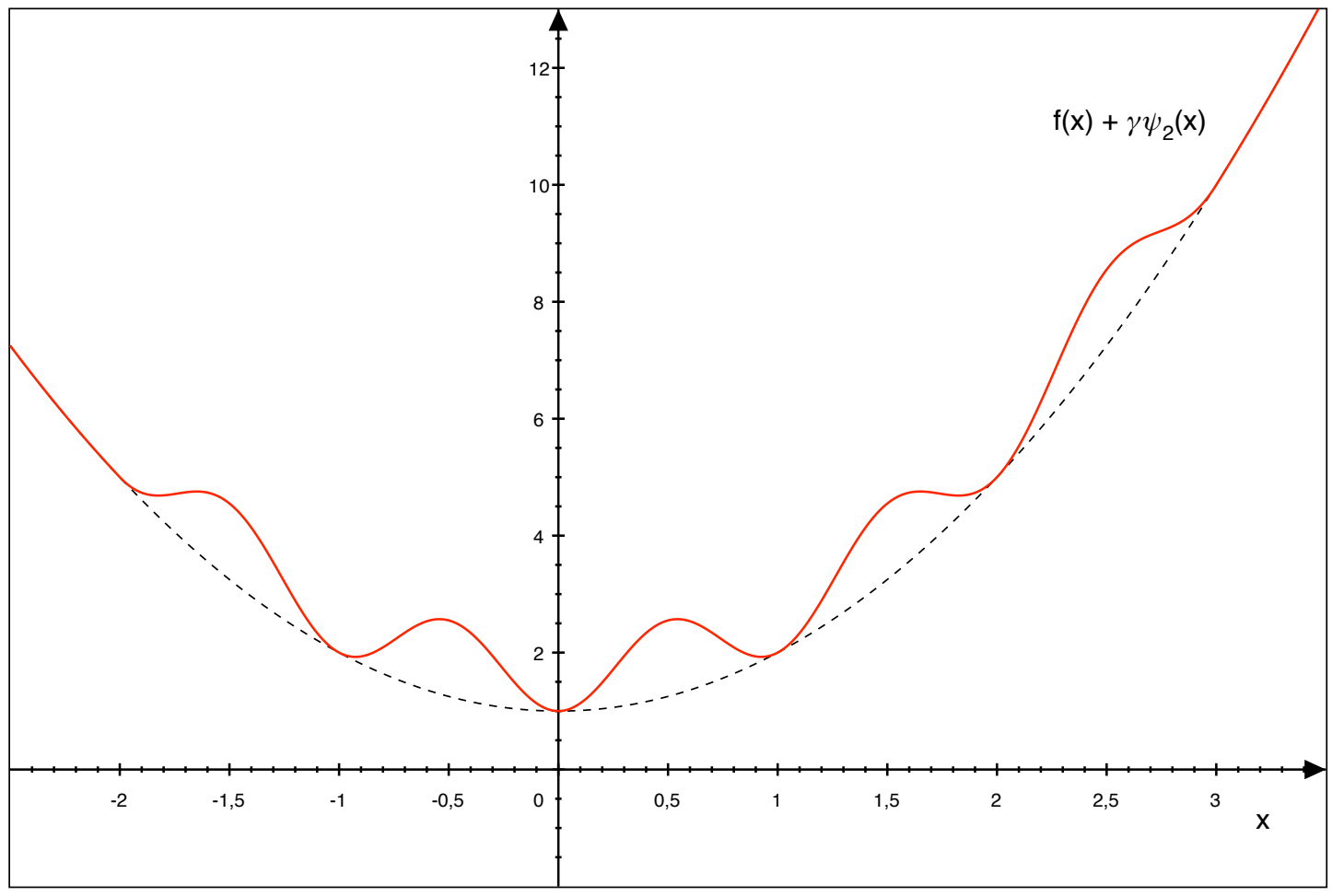

Figura 5.6 - Função objetivo aumentada definida por $x \in \mathcal{X}$, para $\gamma=1,3$. 


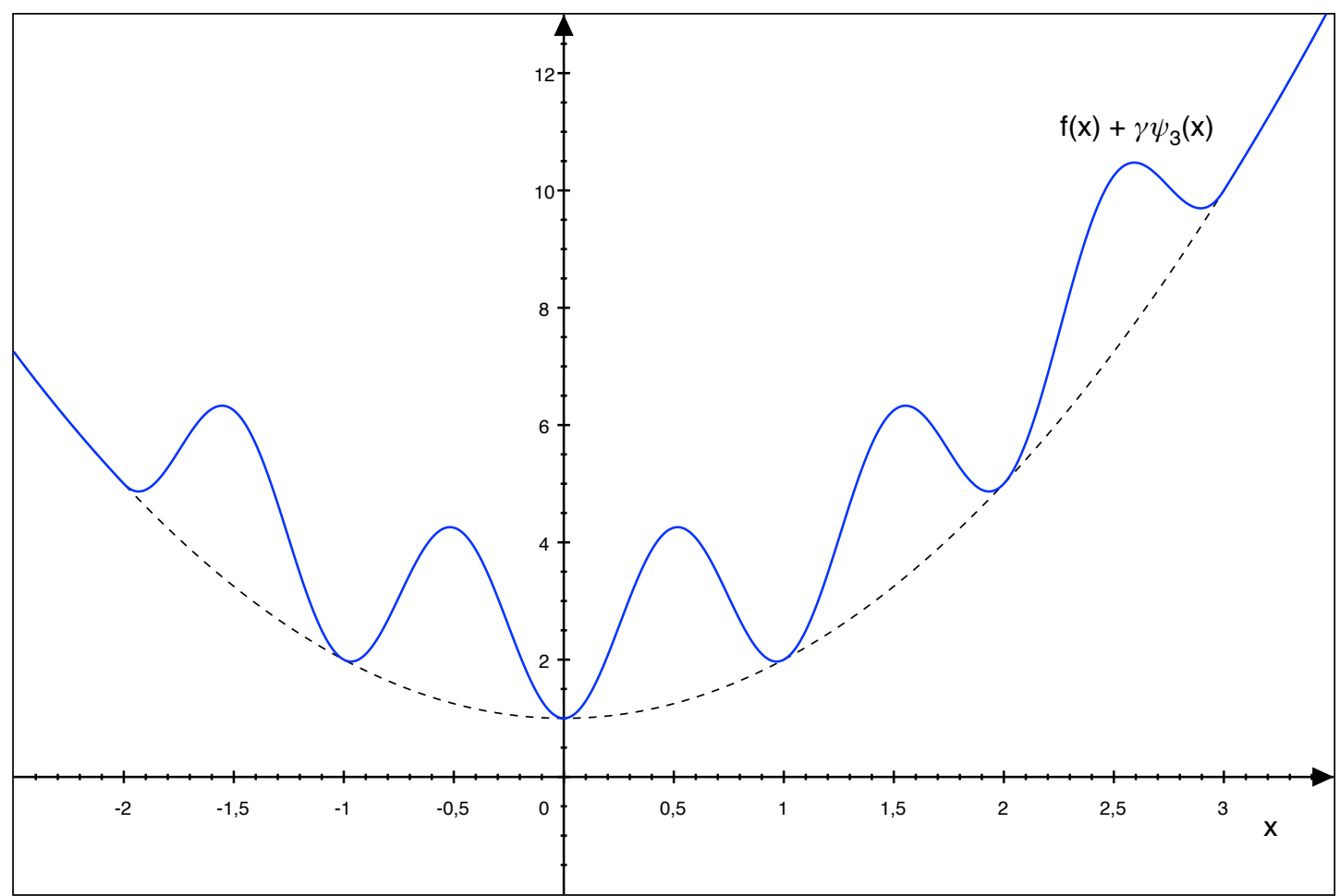

Figura 5.7 - Função objetivo aumentada definida por $x \in \mathcal{X}$, para $\gamma=3,0$.

\subsubsection{O Parâmetro $\alpha$}

É importante ilustrar também o caso em que o conjunto de valores discretos é formado por elementos cuja distância entre dois valores discretos consecutivos não é constante. Para isso, seja $\mathcal{X}^{\prime}=\{-2,0,1,3\} \mid \mathcal{X}^{\prime} \subset \mathcal{X}$ o novo conjunto de valores discretos da variável $x$ do problema (5.6). A representação gráfica deste problema é dada na Figura 5.8. A função $\psi$ é definida da seguinte forma:

$$
\psi(x)=\left\{\begin{array}{lll}
0 & \text { se } \quad x<-2 \\
{\left[\operatorname{sen}\left(\frac{x}{2} \pi+\alpha_{1}\right)\right]^{2 \beta}} & \text { se } \quad-2 \leq x<0 \\
{\left[\operatorname{sen}\left(x \pi+\alpha_{2}\right)\right]^{2 \beta}} & \text { se } \quad 0 \leq x<1 \\
{\left[\operatorname{sen}\left(\frac{x}{2} \pi+\alpha_{3}\right)\right]^{2 \beta}} & \text { se } \quad 1 \leq x \leq 3 \\
0 & \text { se } \quad x>3
\end{array}\right.
$$


sendo os valores de $\alpha_{1}, \alpha_{2}$ e $\alpha_{3}$ que anulam a função $\psi$ em $x \in \mathcal{X}^{\prime}$ dados por:

$$
\begin{aligned}
& \alpha_{1}=0 \\
& \alpha_{2}=0 \\
& \alpha_{3}=\frac{\pi}{2}
\end{aligned}
$$

Nesse caso, a influência do parâmetro $\gamma$ na semelhança entre a função objetivo do problema (5.6) e a função objetivo aumentada é dada nas Figuras 5.9, 5.10 e 5.11.

\subsection{Algoritmo}

1) Dado o problema (5.1), construa a função $\psi(5.2)$ e defina o problema modificado (5.3);

2) Faça $k=0$;

Dê um valor inicial para $x_{1}^{0}$ e $x_{2}^{0}$ e para o parâmetro $\gamma^{0}$;

Especifique um valor para o fator $\tau>1$ e a tolerância $\xi$;

3) Resolva o problema (5.3), com $\gamma^{k}$ fixo, utilizando o método da FLBMP;

4) Admita $x_{1}^{k}$ e $x_{2}^{k}$ como uma solução e vá para o passo 5 ;

5) Se $\left|x_{2_{i}}^{k}-x_{2_{i}}^{\prime}\right|<\xi$, para $i=1, \cdots, m_{2}$, pare;

Caso contrário, atualize o parâmetro $\gamma$ por (5.4), faça $k=k+1$, e volte para o passo 3. 


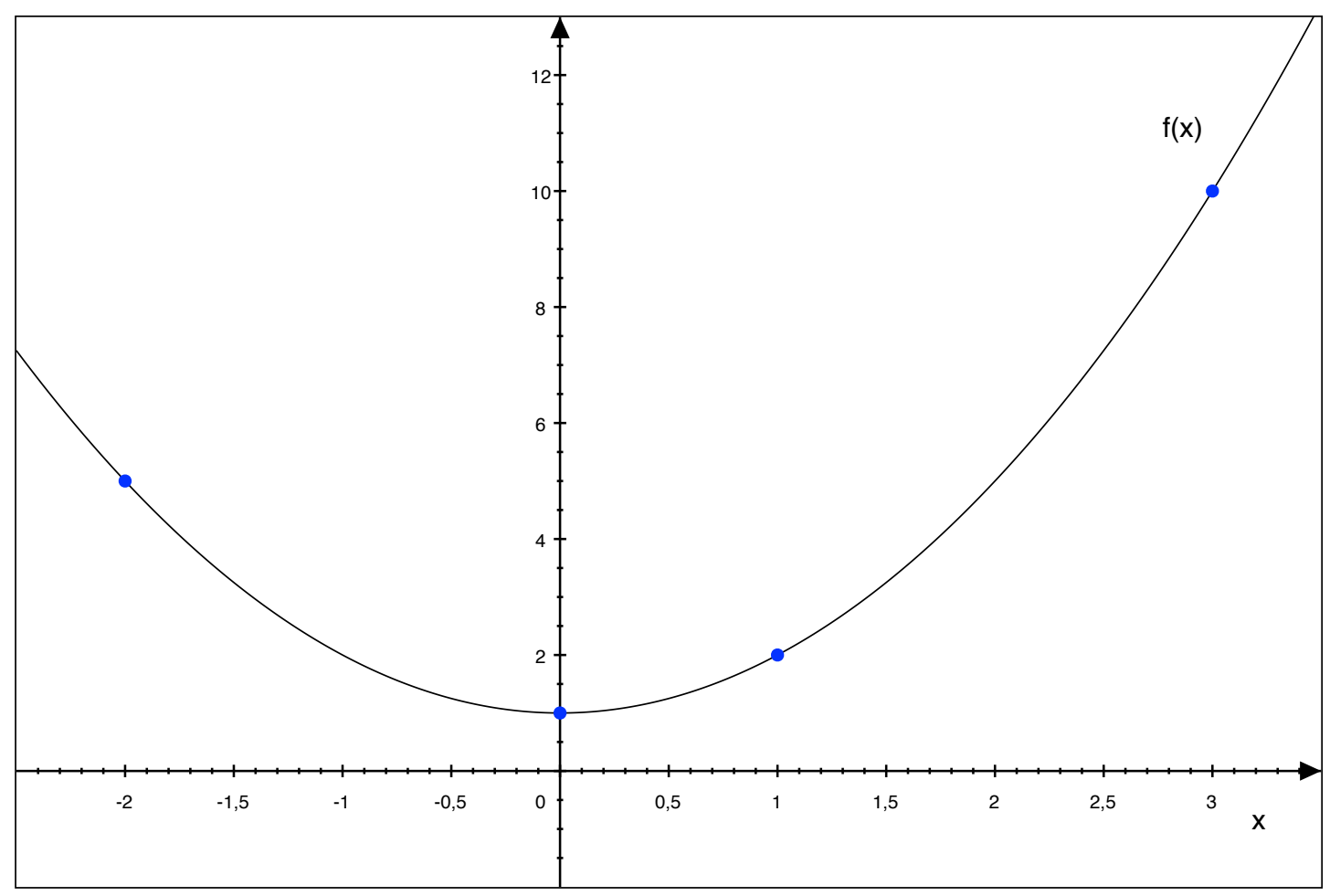

Figura 5.8 - Representação gráfica do problema (5.6), onde $x \in \mathcal{X}^{\prime}$.

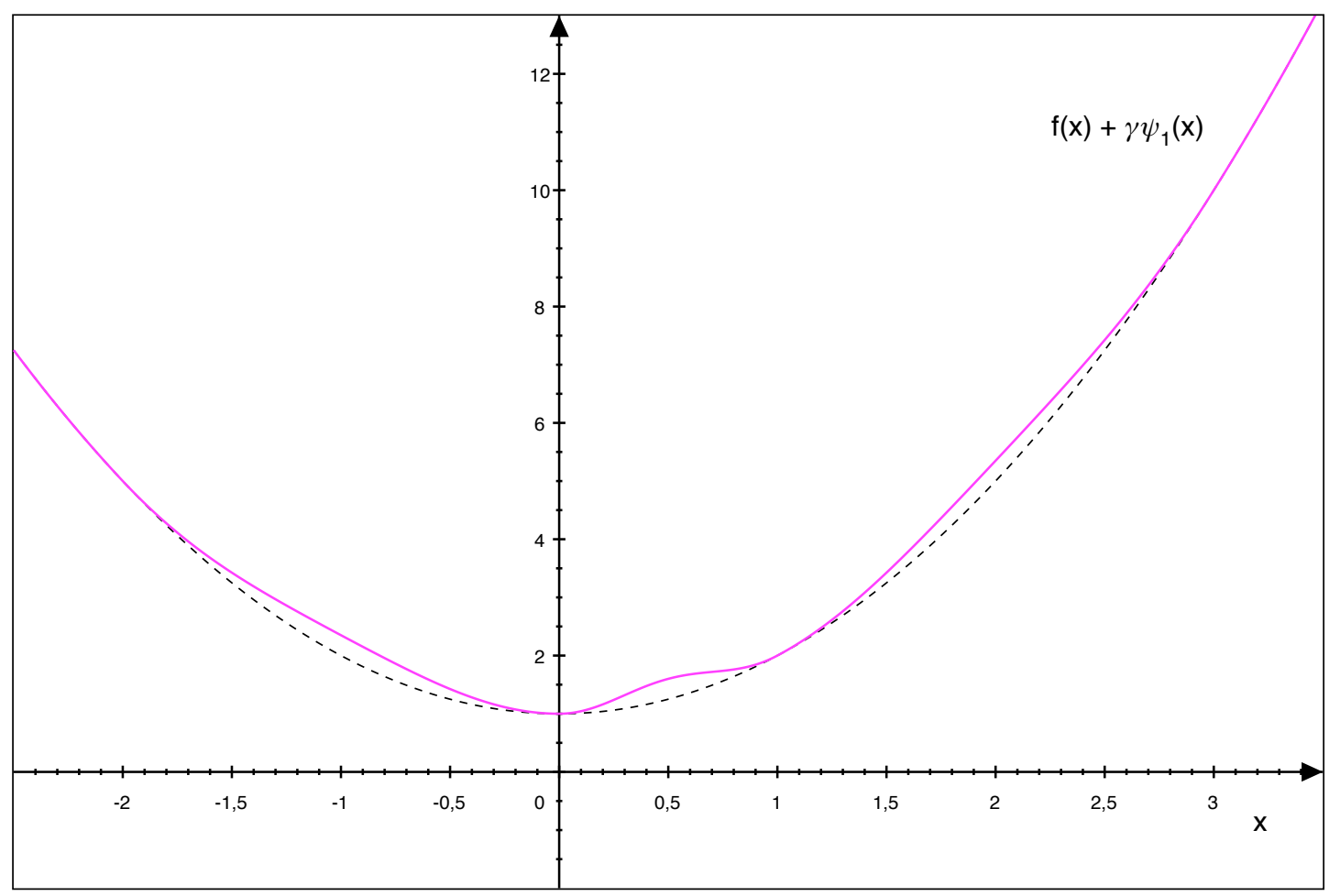

Figura 5.9 - Função objetivo aumentada definida por $x \in \mathcal{X}^{\prime}$, para $\gamma=0,3$. 


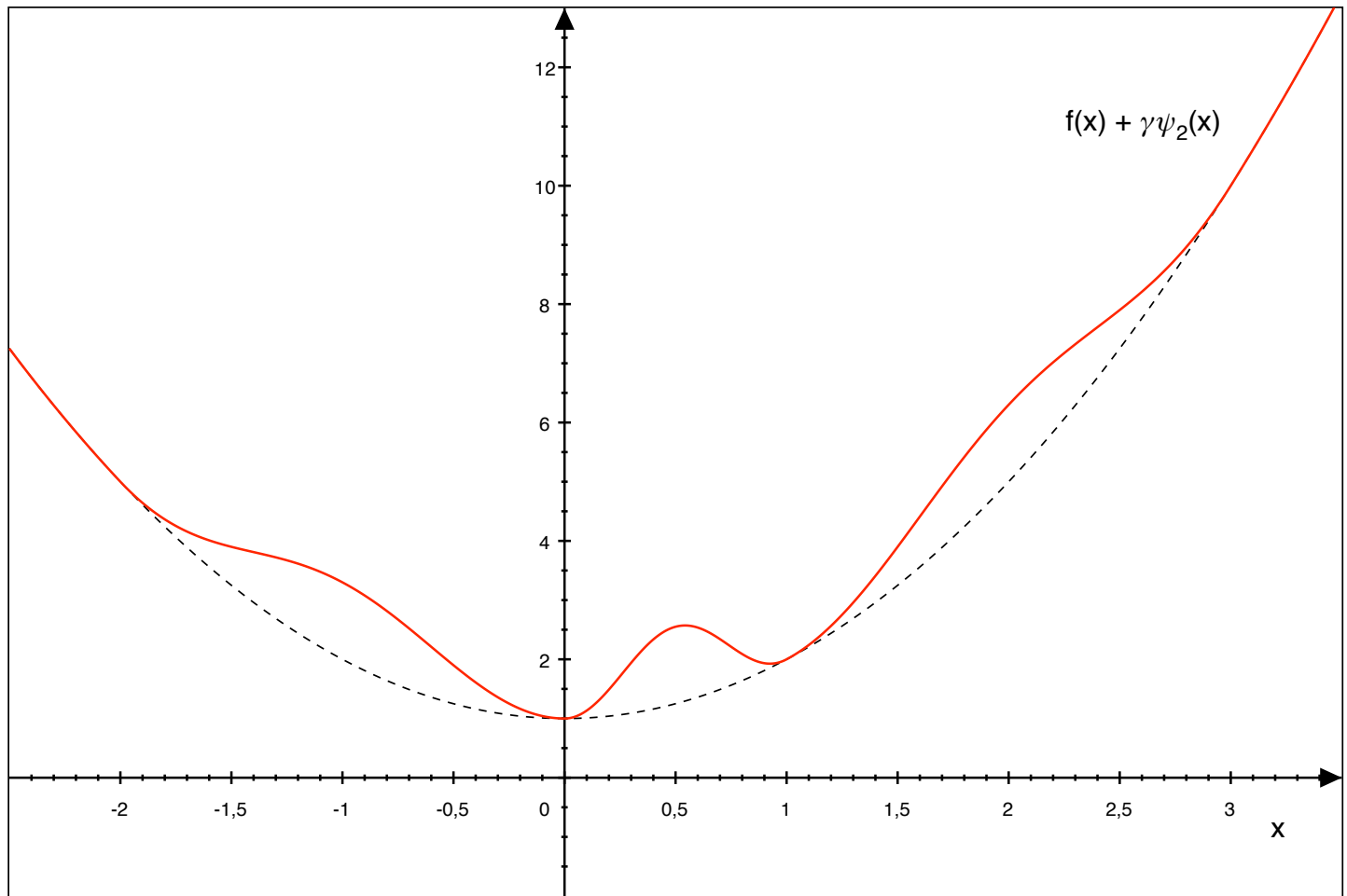

Figura 5.10 - Função objetivo aumentada definida por $x \in \mathcal{X}^{\prime}$, para $\gamma=1,3$.

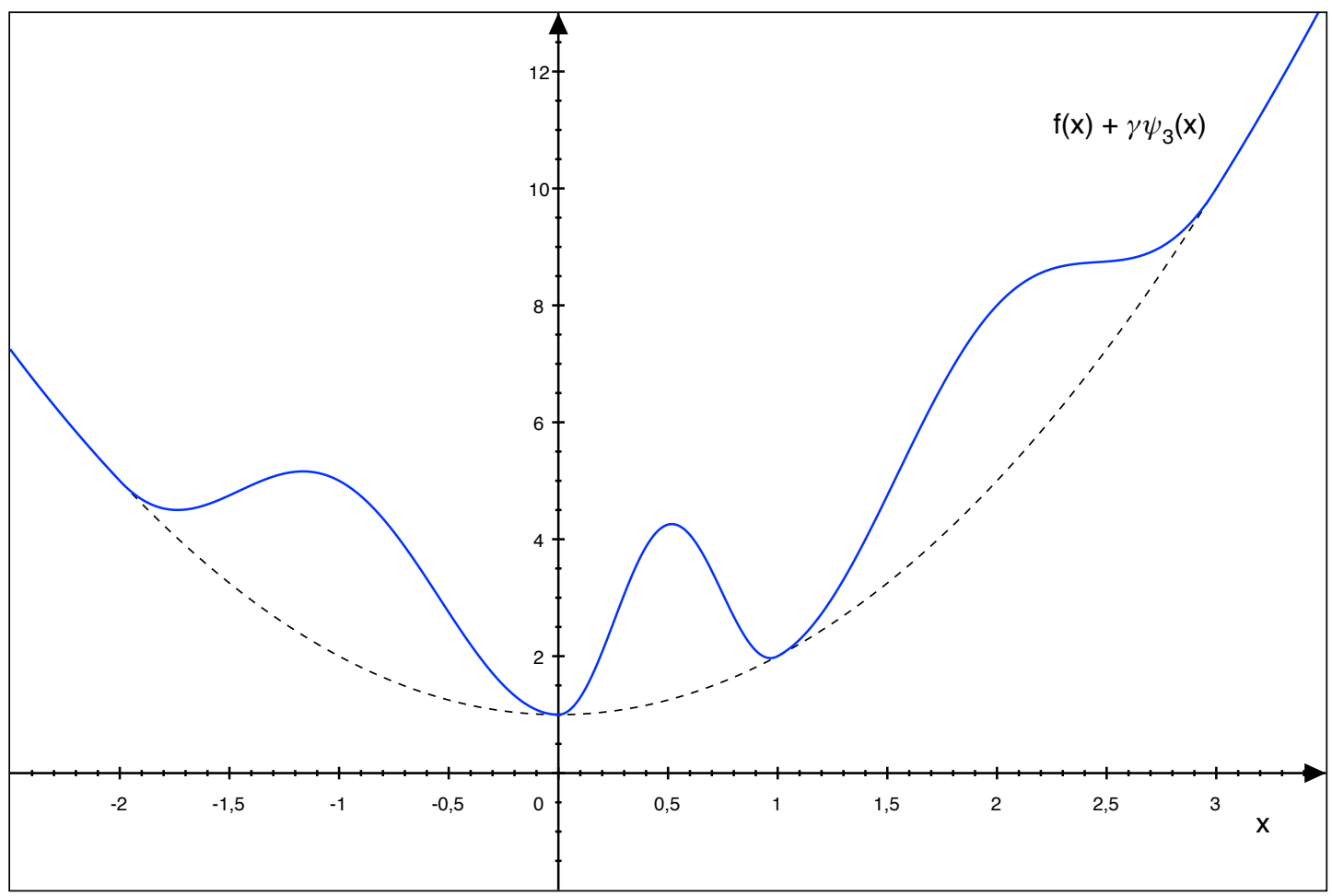

Figura 5.11 - Função objetivo aumentada definida por $x \in \mathcal{X}^{\prime}$, para $\gamma=3,0$. 


\subsection{Exemplo Numérico}

Para exemplificar o uso ao algoritmo da FLBMP-discreto na resolução de problemas de PNL com variáveis contínuas e discretas, considere o seguinte problema, adaptado de Soler (2011):

$$
\begin{array}{ll}
\min & \left(x_{1}-2\right)^{4}+\left(x_{1}-2 x_{2}\right)^{2} \\
\text { s.a: } & x_{1}+x_{2}=3 \\
& x_{1}^{2}-x_{2} \leq 0 \\
& x_{1} \in \mathcal{X}
\end{array}
$$

onde $\mathcal{X}=\{0,8 ; 0,95 ; 1,1 ; 1,25 ; 1,4 ; 1,55 ; 1,7\}$.

De acordo com o algoritmo da FLBMP-discreto, a função $\psi$ é dada por:

$$
\psi\left(x_{1}\right)=\left\{\begin{array}{lll}
0 & \text { se } x_{1}<0,8 \\
{\left[\operatorname{sen}\left(\frac{x_{1}}{0,15} \pi+\alpha\right)\right]^{2 \beta}} & \text { se } 0,8 \leq x_{1} \leq 1,7 \\
0 & \text { se } \quad x_{1}>1,7
\end{array}\right.
$$

onde $\beta=1$ e $\alpha=\frac{2}{3} \pi$.

Dessa forma, o problema modificado é dado, portanto, por:

$$
\begin{array}{ll}
\min & \left(x_{1}-2\right)^{4}+\left(x_{1}-2 x_{2}\right)^{2}+\gamma\left[\operatorname{sen}\left(\frac{x_{1}}{0,15} \pi+\frac{2}{3} \pi\right)\right]^{2} \\
\text { s.a: } & x_{1}+x_{2}=3 \\
& x_{1}^{2}-x_{2} \leq 0 \\
& 0,8 \leq x_{1} \leq 1,7
\end{array}
$$


Por fim, de acordo com o método da FLBMP, o problema (5.12) é modificado da seguinte forma:

$$
\begin{aligned}
& \min \left(x_{1}-2\right)^{4}+\left(x_{1}-2 x_{2}\right)^{2}+\gamma\left[\operatorname{sen}\left(\frac{x_{1}}{0,15} \pi+\frac{2}{3} \pi\right)\right]^{2} \\
& \text { s.a: } \quad x_{1}+x_{2}-3=0 \\
& x_{1}^{2}-x_{2}+s_{1}=0 \\
& 0,8-x_{1}+s_{2}=0 \\
& x_{1}-1,7+s_{3}=0 \\
& s_{i} \geq 0 \quad i=1, \cdots, 3
\end{aligned}
$$

A FLBMP associada ao problema (5.13) é dada por:

$$
\begin{aligned}
\mathcal{L}(x, s, \lambda, \pi)= & \left(x_{1}-2\right)^{4}+\left(x_{1}-2 x_{2}\right)^{2}+\gamma\left[\operatorname{sen}\left(\frac{x_{1}}{0,15} \pi+\frac{2}{3} \pi\right)\right]^{2}- \\
& \mu\left[\sigma_{1} \phi\left(s_{1}\right)+\sigma_{2} \phi\left(s_{2}\right)+\sigma_{3} \phi\left(s_{3}\right)\right]+ \\
& \lambda_{1}\left(x_{1}+x_{2}-3\right)+\pi_{1}\left(x_{1}^{2}-x_{2}+s_{1}\right)+ \\
& \pi_{2}\left(0,8-x_{1}+s_{2}\right)+\pi_{3}\left(x_{1}-1,7+s_{3}\right)
\end{aligned}
$$

Para resolver esse problema, o método da FLBMP foi inicializado com o ponto $x_{1}=0,9$ e $x_{2}=0,8$; as variáveis de folga $s_{1}, s_{2}$ e $s_{3}$ foram inicializadas, respectivamente, a partir das restrições $(5.13 \mathrm{c}),(5.13 \mathrm{~d})$ e (5.13e). Os multiplicadores de Lagrange $\lambda, \pi$ e $\sigma$ foram inicializados, respectivamente, por 0,1 e 1 . Neste teste, considerou-se $\mu^{0}=10^{-1} \mathrm{e}$ as tolerâncias para convergência foram $\xi_{1}=10^{-5}, \xi_{2}=10^{-4}$ e $\xi_{3}=10^{-3}$.

No algoritmo da FLBMP-discreto, considerou-se $\gamma^{0}=0,1$, com $\tau=3$, e $\xi=10^{-4}$.

O processo de convergência do algoritmo da FLBMP-discreto é apresentado nas Tabelas 5.1 e 5.2. As cinco primeiras iterações do algoritmo da FLBMP-discreto são representadas graficamente nas Figuras 5.12, 5.13, 5.14, 5.15 e 5.16, onde os pontos alaranjados representam soluções discretas do problema (5.10). 
Tabela 5.1 - Convergência do algoritmo da FLBMP-discreto aplicado ao problema (5.10) Função objetivo, variáveis primais e parâmetro $\mu$.

\begin{tabular}{|c|c|c|c|c|c|c|c|c|c|}
\hline $\begin{array}{c}\text { Iteração } \\
\text { FLBMP-D }\end{array}$ & $\begin{array}{l}\text { Iteração } \\
\text { FLBMP }\end{array}$ & F. Obj. & $x_{1}$ & $x_{2}$ & $s_{1}$ & $s_{2}$ & $s_{3}$ & $\mu$ & $\gamma$ \\
\hline 0 & 0 & 1,9541 & 0,9000 & 0,8000 & $-0,0100$ & 0,1000 & 0,8000 & $10^{-1}$ & 0,10 \\
\hline \multirow[t]{4}{*}{1} & 1 & 4,3386 & 1,3227 & 1,6773 & $-0,0724$ & 0,5227 & 0,3773 & $10^{-1}$ & 0,10 \\
\hline & 2 & 4,5770 & 1,3053 & 1,6947 & $-0,0089$ & 0,5053 & 0,3947 & $10^{-2}$ & 0,10 \\
\hline & 3 & 4,6045 & 1,3033 & 1,6967 & $-0,0018$ & 0,5033 & 0,3967 & $10^{-3}$ & 0,10 \\
\hline & 4 & 4,6114 & 1,3028 & 1,6972 & 0,0000 & 0,5028 & 0,3972 & $10^{-4}$ & 0,10 \\
\hline \multirow[t]{5}{*}{2} & 1 & 4,8162 & 1,2882 & 1,7118 & 0,0522 & 0,4882 & 0,4118 & $10^{-1}$ & 0,30 \\
\hline & 2 & 4,5762 & 1,3053 & 1,6947 & $-0,0091$ & 0,5053 & 0,3947 & $10^{-2}$ & 0,30 \\
\hline & 3 & 4,6116 & 1,3028 & 1,6972 & 0,0000 & 0,5028 & 0,3972 & $10^{-3}$ & 0,30 \\
\hline & 4 & 4,6072 & 1,3031 & 1,6969 & $-0,0011$ & 0,5031 & 0,3969 & $10^{-4}$ & 0,30 \\
\hline & 5 & 4,6114 & 1,3028 & 1,6972 & 0,0000 & 0,5028 & 0,3972 & $10^{-5}$ & 0,30 \\
\hline \multirow[t]{3}{*}{3} & 1 & 5,1533 & 1,2650 & 1,7350 & 0,1347 & 0,4650 & 0,4350 & $10^{-1}$ & 0,90 \\
\hline & 2 & 5,0621 & 1,2712 & 1,7288 & 0,1128 & 0,4712 & 0,4288 & $10^{-2}$ & 0,90 \\
\hline & 3 & 5,0613 & 1,2713 & 1,7287 & 0,1126 & 0,4713 & 0,4287 & $10^{-3}$ & 0,90 \\
\hline 4 & 1 & 5,2819 & 1,2564 & 1,7436 & 0,1650 & 0,4564 & 0,4436 & $10^{-1}$ & 2,70 \\
\hline 5 & 1 & 5,3466 & 1,2521 & 1,7479 & 0,1800 & 0,4521 & 0,4479 & $10^{-1}$ & 8,10 \\
\hline 6 & 1 & 5,3681 & 1,2507 & 1,7493 & 0,1850 & 0,4507 & 0,4493 & $10^{-1}$ & 24,30 \\
\hline 7 & 1 & 5,3753 & 1,2502 & 1,7498 & 0,1867 & 0,4502 & 0,4498 & $10^{-1}$ & 72,90 \\
\hline 8 & 1 & 5,3777 & 1,2501 & 1,7499 & 0,1872 & 0,4501 & 0,4499 & $10^{-1}$ & 218,70 \\
\hline 9 & 1 & 5,3785 & 1,2500 & 1,7500 & 0,1874 & 0,4500 & 0,4500 & $10^{-1}$ & 656,10 \\
\hline
\end{tabular}


Tabela 5.2 - Convergência do algoritmo da FLBMP-discreto aplicado ao problema (5.10) Variáveis duais.

\begin{tabular}{|c|c|c|c|c|c|c|c|c|}
\hline $\begin{array}{l}\text { Iteração } \\
\text { FLBMP-D }\end{array}$ & $\begin{array}{l}\text { Iteração } \\
\text { FLBMP }\end{array}$ & $\lambda$ & $\pi_{1}$ & $\pi_{2}$ & $\pi_{3}$ & $\sigma_{1}$ & $\sigma_{2}$ & $\sigma_{3}$ \\
\hline 0 & 0 & 0,0000 & 1,0000 & 1,0000 & 1,0000 & 1,0000 & 1,0000 & 1,0000 \\
\hline \multirow[t]{4}{*}{1} & 1 & $-4,5102$ & 3,6171 & 0,1606 & 0,2095 & 1,0000 & 1,0000 & 1,0000 \\
\hline & 2 & $-4,9288$ & 3,4081 & 0,0003 & 0,0005 & 0,3617 & 0,0161 & 0,0210 \\
\hline & 3 & $-4,9668$ & 3,3939 & 0,0000 & 0,0000 & 0,0341 & 0,0000 & 0,0000 \\
\hline & 4 & $-4,9759$ & 3,3908 & 0,0000 & 0,0000 & 3,3939 & 0,0000 & 0,0000 \\
\hline \multirow[t]{5}{*}{2} & 1 & $-6,3116$ & 2,2296 & 0,0000 & 0,0000 & 3,3939 & 0,0000 & 0,0000 \\
\hline & 2 & $-5,7796$ & 2,5567 & 0,0000 & 0,0000 & 0,2230 & 0,0000 & 0,0000 \\
\hline & 3 & $-5,9084$ & 2,4585 & 0,0000 & 0,0000 & 2,5567 & 0,0000 & 0,0000 \\
\hline & 4 & $-5,8934$ & 2,4697 & 0,0000 & 0,0000 & 0,0025 & 0,0000 & 0,0000 \\
\hline & 5 & $-5,9078$ & 2,4589 & 0,0000 & 0,0000 & 2,4697 & 0,0000 & 0,0000 \\
\hline \multirow[t]{3}{*}{3} & 1 & $-7,7671$ & 1,0525 & 0,0000 & 0,0000 & 2,4697 & 0,0000 & 0,0000 \\
\hline & 2 & $-8,7367$ & 0,0086 & 0,0000 & 0,0000 & 0,1052 & 0,0000 & 0,0000 \\
\hline & 3 & $-8,7446$ & 0,0000 & 0,0000 & 0,0000 & 0,0001 & 0,0000 & 0,0000 \\
\hline 4 & 1 & $-8,9229$ & 0,0000 & 0,0000 & 0,0000 & 0,0001 & 0,0000 & 0,0000 \\
\hline 5 & 1 & $-8,9744$ & 0,0000 & 0,0000 & 0,0000 & 0,0001 & 0,0000 & 0,0000 \\
\hline 6 & 1 & $-8,9914$ & 0,0000 & 0,0000 & 0,0000 & 0,0001 & 0,0000 & 0,0000 \\
\hline 7 & 1 & $-8,9971$ & 0,0000 & 0,0000 & 0,0000 & 0,0001 & 0,0000 & 0,0000 \\
\hline 8 & 1 & $-8,9990$ & 0,0000 & 0,0000 & 0,0000 & 0,0001 & 0,0000 & 0,0000 \\
\hline 9 & 1 & $-8,9997$ & 0,0000 & 0,0000 & 0,0000 & 0,0001 & 0,0000 & 0,0000 \\
\hline
\end{tabular}




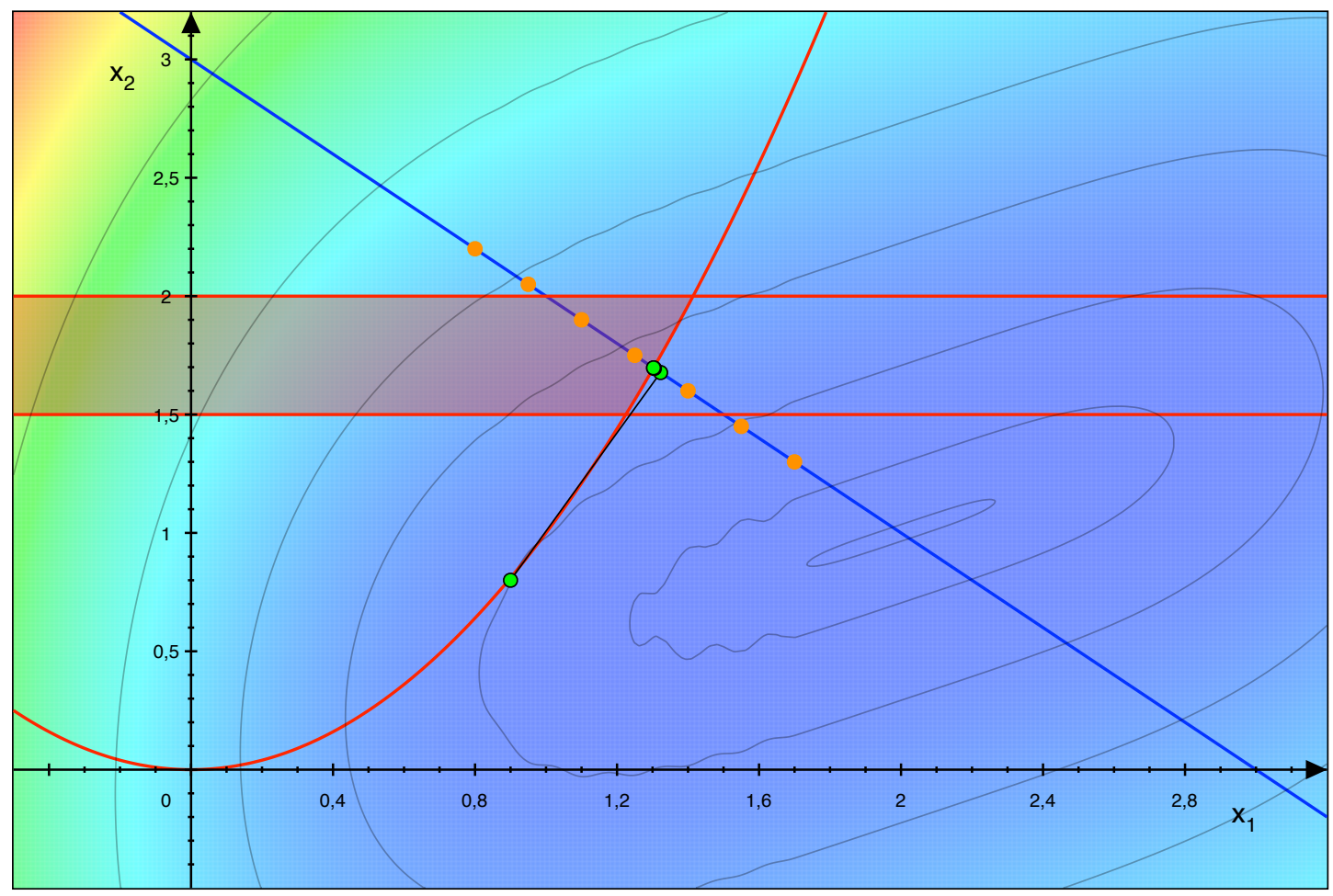

Figura 5.12 - $1^{a}$ iteração do algoritmo da FLBMP-discreto aplicado ao problema (5.10) Convergência do método da FLBMP aplicado ao problema modificado (5.12).

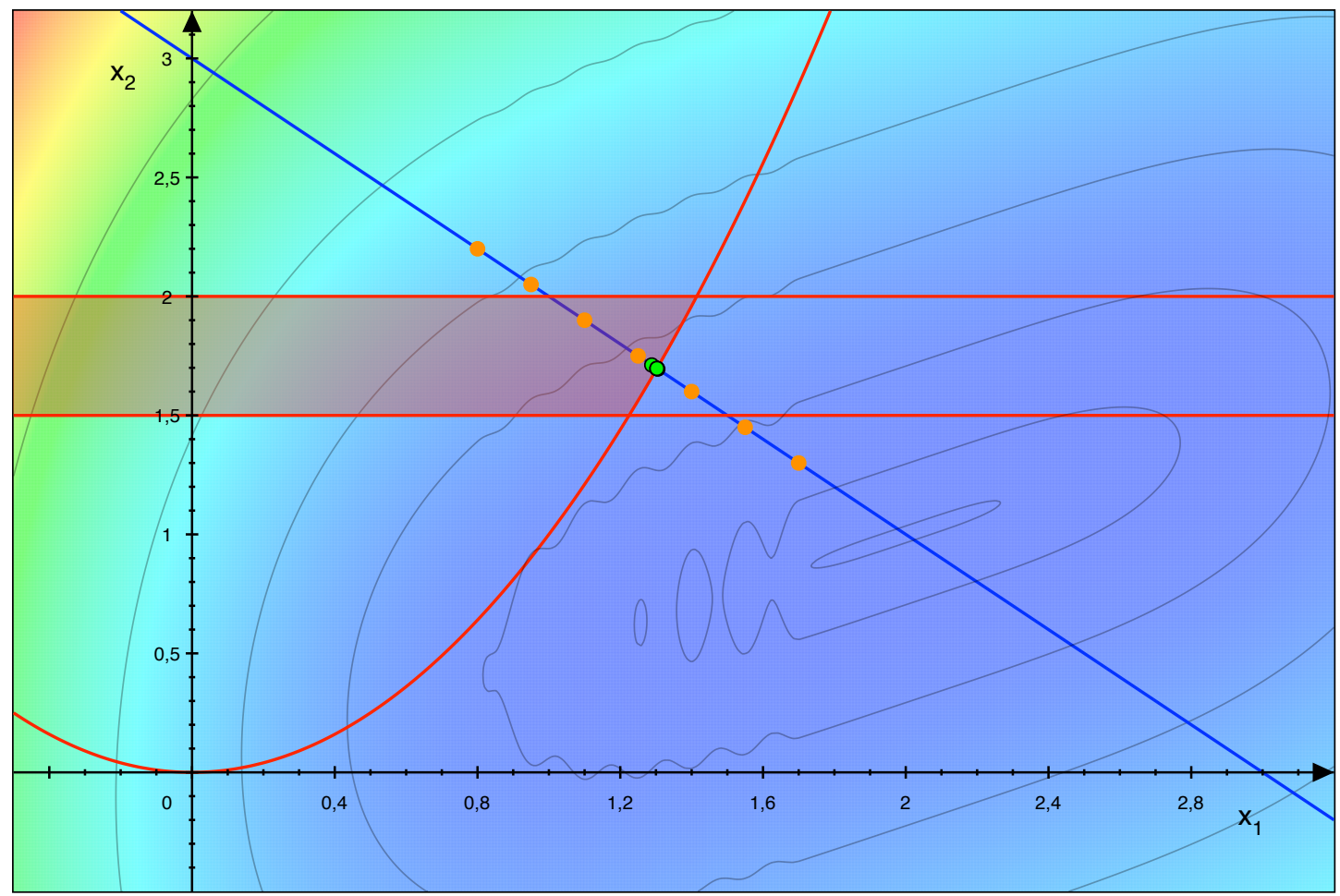

Figura $5.13-2^{a}$ iteração do algoritmo da FLBMP-discreto aplicado ao problema (5.10) Convergência do método da FLBMP aplicado ao problema modificado (5.12). 


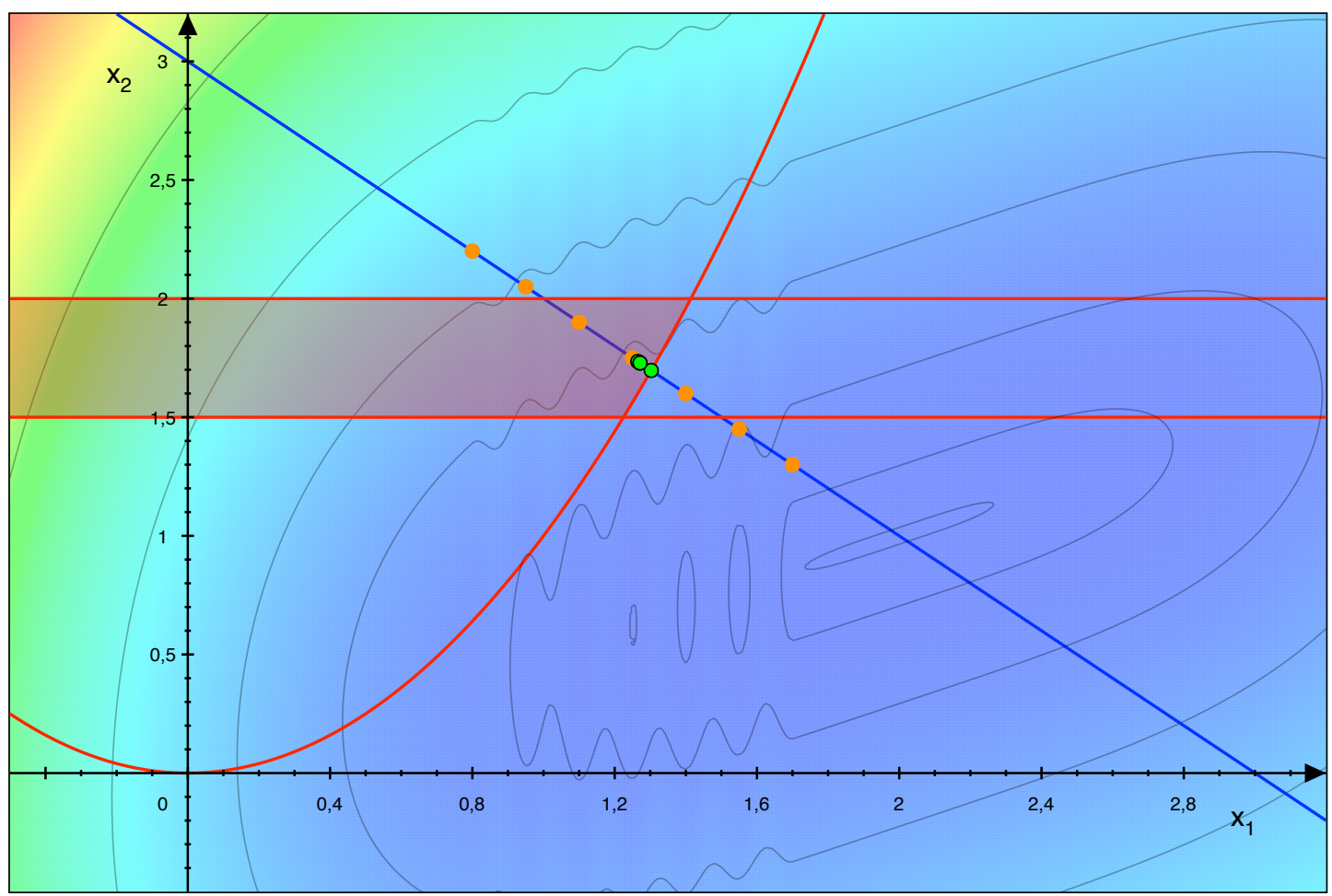

Figura 5.14 - $3^{a}$ iteração do algoritmo da FLBMP-discreto aplicado ao problema (5.10) Convergência do método da FLBMP aplicado ao problema modificado (5.12).

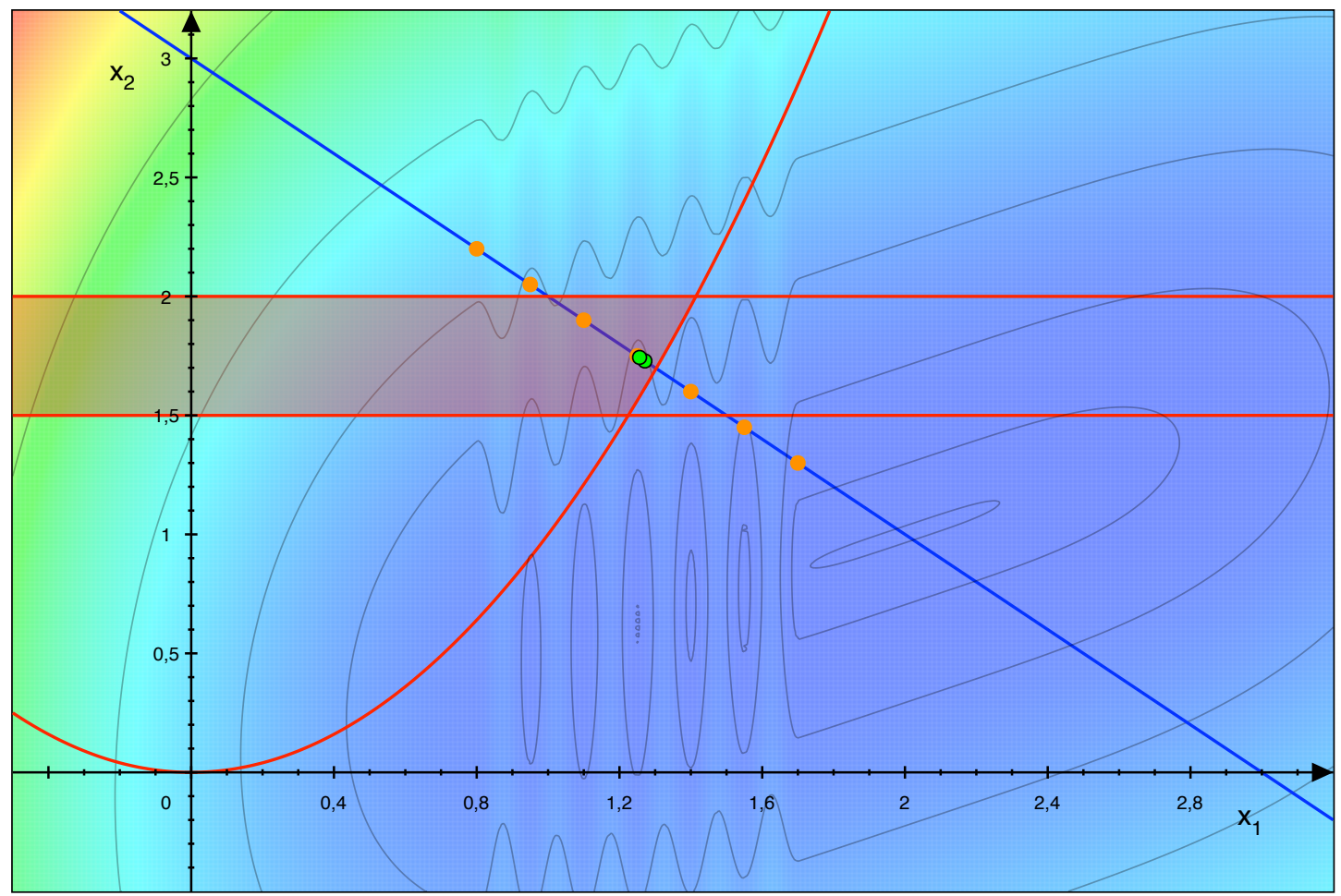

Figura 5.15 - $4^{a}$ iteração do algoritmo da FLBMP-discreto aplicado ao problema (5.10) Convergência do método da FLBMP aplicado ao problema modificado (5.12). 


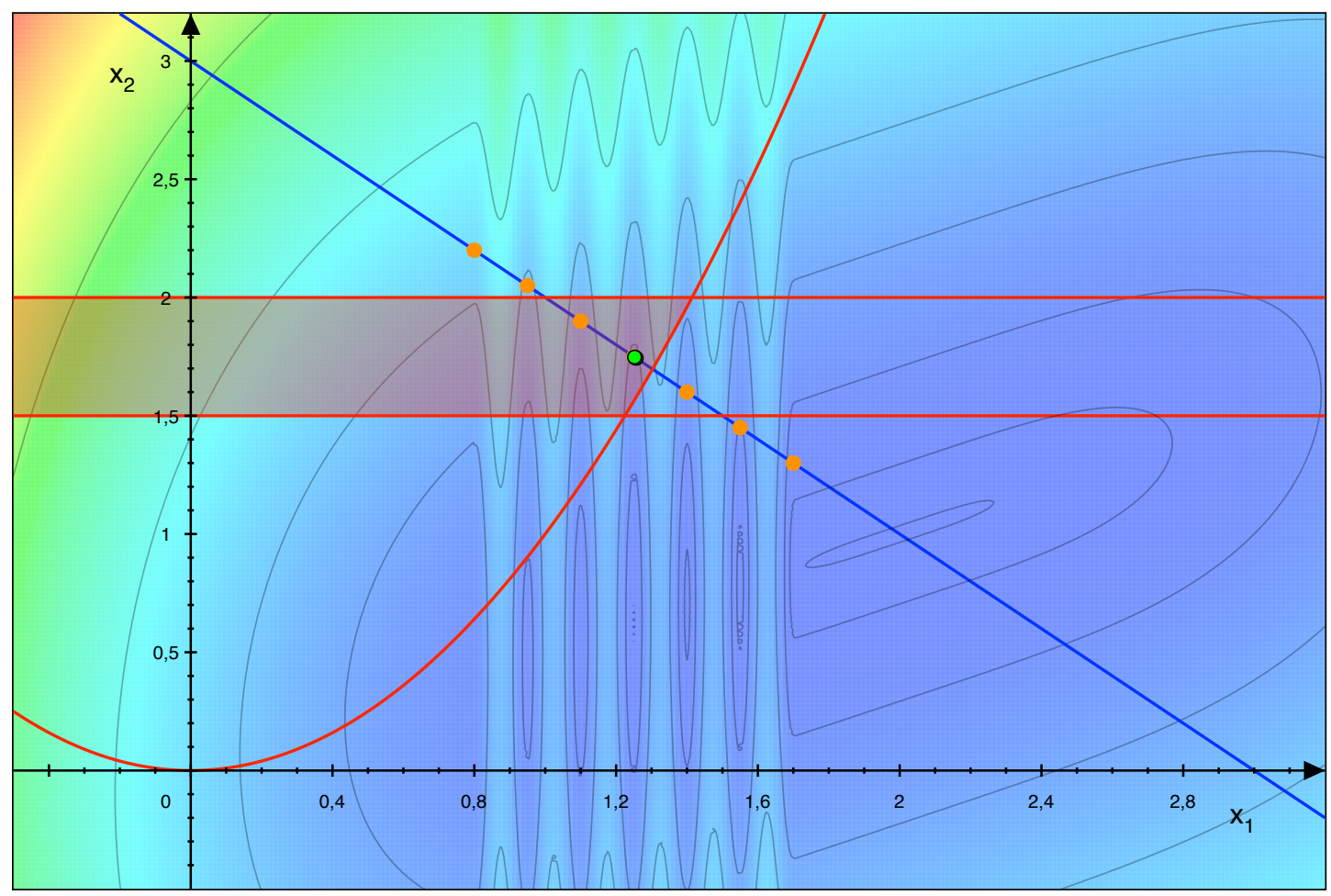

Figura 5.16 - $5^{a}$ iteração do algoritmo da FLBMP-discreto aplicado ao problema (5.10) Convergência do método da FLBMP aplicado ao problema modificado (5.12). 


\section{Capítulo 6}

\section{Problemas de PNL com Restrições de Complementaridade}

Neste capítulo, o método da FLBMP é aplicado à resolução de problemas de PNL com restrições de complementaridade. Para isso, são apresentados, primeiramente, conceitos básicos de problemas de complementaridade. Em seguida, são apresentadas as modificações necessárias para transformar o problema de PNL com restrições de complementaridade em um problema de PNL equivalente.

\subsection{Problemas de Complementaridade}

\subsubsection{Condição de Complementaridade}

A condição de complementaridade entre duas variáveis $a$ e $b$ pode ser representada matematicamente pelo seu produto igual a zero, ou seja:

$$
a b=0
$$

A condição (6.1) pode ser satisfeita de três formas distintas: 
1) $a=0$ e $b \neq 0$

2) $a \neq 0$ e $b=0$;

3) e $a=0$ e $b=0$.

As duas primeiras soluções satisfazem a condição de complementaridade de forma estrita, pois somente uma das variáveis, $a$ ou $b$, é igual a zero. A terceira solução satisfaz a condição de complementaridade de forma não estrita, pois tanto $a$ quanto $b$ são iguais a zero.

Em muitos problemas, a condição de complementaridade entre duas grandezas é comumente expressa pelo símbolo " $\perp$ ". Portanto:

$$
a \perp b \quad \Leftrightarrow \quad a b=0
$$

A condição de complementaridade também é definida para vetores. Nesse caso, sejam $a$ e $b$ vetores de dimensão $n$. A condição de complementaridade entre $a$ e $b$ pode ser representada pelo o produto escalar nulo entre esses vetores, tal que o produto entre o $i$-ésimo elemento de $a$ e o $i$-ésimo elemento de $b$ seja igual a zero. Matematicamente, a condição de complementaridade entre esses vetores é escrita da seguinte forma:

$$
a \perp b \quad \Rightarrow \quad a^{T} b=0 \quad \mid \quad a_{i} b_{i}=0, i=1, \cdots, n
$$

\subsubsection{Problemas de Complementaridade}

Seja $c: \mathbb{R}^{n} \rightarrow \mathbb{R}^{n}$. Um problema de complementaridade consiste em encontrar $x \in \mathbb{R}^{n}$ tal que:

$$
0 \leq x \perp c(x) \geq 0
$$

Se $c$ for uma função afim, (6.4) é um problema de complementaridade linear, caso contrário, este é um problema de complementaridade não-linear. A representação gráfica do problema (6.4) é dada na Figura 6.1. 


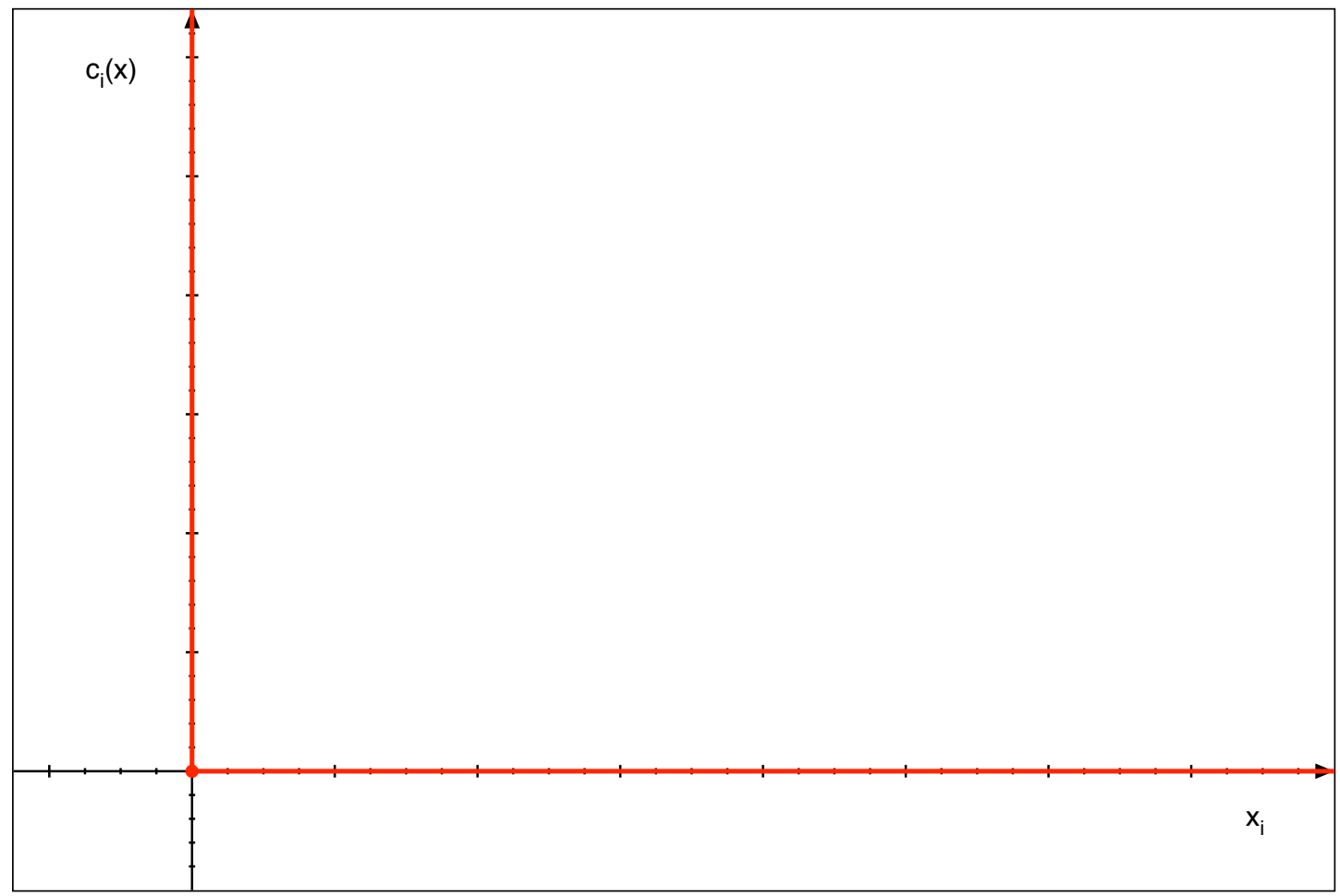

Figura 6.1 - Representação gráfica do problema de complementaridade (6.4).

De acordo com (6.3), o problema (6.4) pode ser reescrito da seguinte forma:

$$
\begin{aligned}
& x_{i} c_{i}(x)=0 \\
& c_{i}(x) \geq 0 \\
& x_{i} \geq 0
\end{aligned}
$$

para $i=1, \cdots, n$.

\section{Problemas de Complementaridade com Limites Inferiores e Superiores}

Em muitos problemas de aplicação prática (FERRIS; PANG, 1997), o problema de complementaridade consiste em encontrar um vetor $x \in \mathcal{X} \subset \mathbb{R}^{n}$ tal que:

$$
x^{\min } \leq x \leq x^{\max } \perp c(x)
$$

onde $\mathcal{X}=\left\{x \in \mathbb{R}^{n}: x^{\min } \leq x \leq x^{\max }\right\}$ e $c: \mathbb{R}^{n} \rightarrow \mathbb{R}^{n}$. 


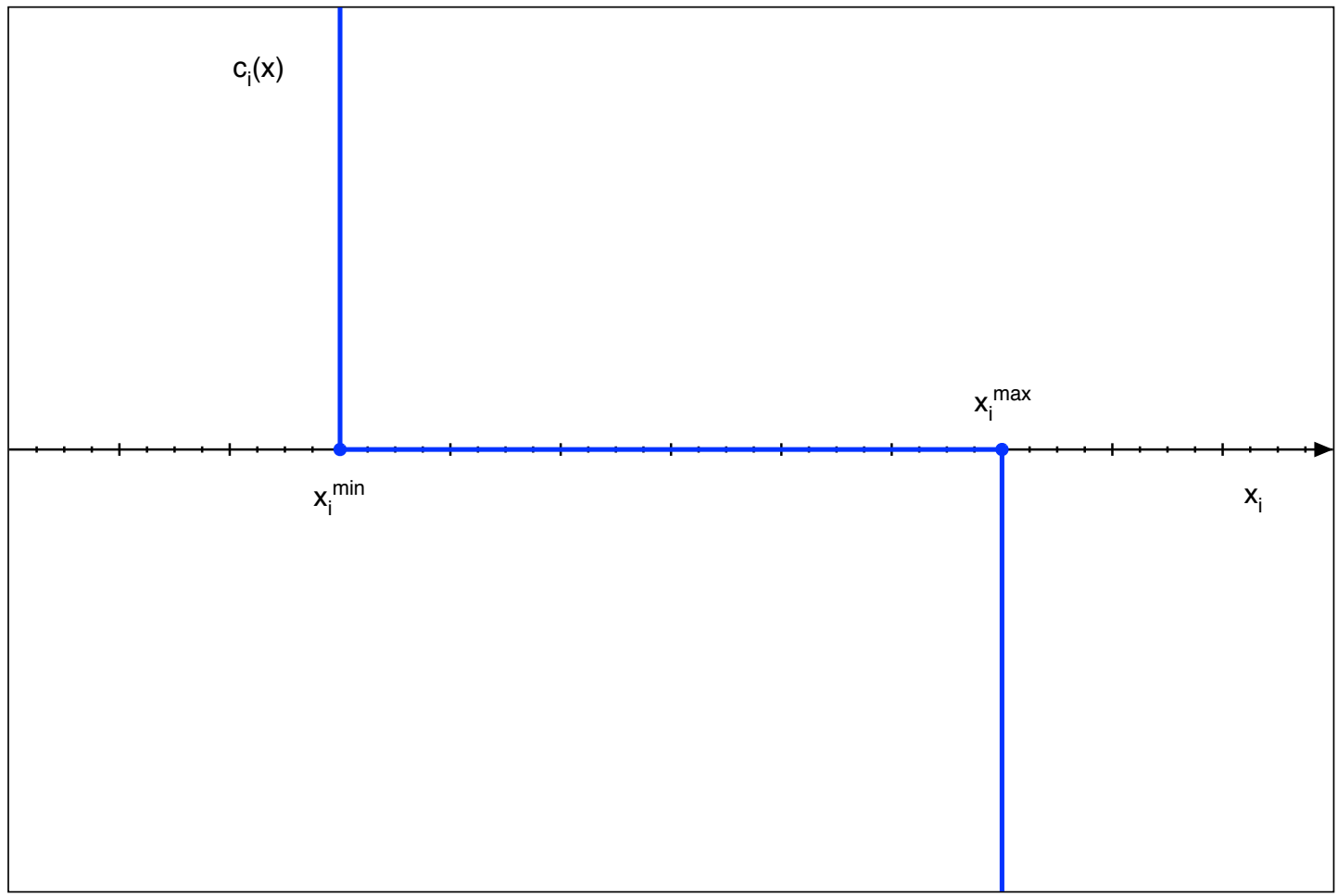

Figura 6.2 - Representação gráfica do problema de complementaridade (6.6).

Ou seja, (6.6) significa que:

$$
\begin{array}{llll}
\text { se } \quad x=x^{\text {min }} & \Rightarrow & c(x) \geq 0 ; \\
\text { se } \quad x^{\text {min }}<x<x^{\text {max }} & \Rightarrow & c(x)=0 ; \\
\text { se } \quad x=x^{\text {max }} & \Rightarrow & c(x) \leq 0 .
\end{array}
$$

A representação gráfica do problema (6.6) é dada na Figura 6.2.

Alternativamente, problemas de complementaridade como (6.6) podem ser reformulados como uma associação de dois problemas de complementaridade do tipo (6.4). O problema (6.6) é modificado da seguinte forma:

1) as restrições canalizadas são desmembradas em desigualdades simples:

$$
0 \leq x-x^{\min } \quad \text { e } \quad 0 \leq x^{\max }-x
$$


2) o valor de $c$ em $x$ passa a ser especificado em função das variáveis auxiliares não negativas $c_{a}, c_{b} \in \mathbb{R}^{n}$ :

$$
c(x)=c_{a}-c_{b}
$$

3) e, finalmente, o problema (6.6) é reescrito como uma associação de dois problemas de complementaridade do tipo (6.4) da seguinte forma:

$$
\begin{aligned}
& c(x)=c_{a}-c_{b} \\
& 0 \leq\left(x-x^{\text {min }}\right) \perp c_{a} \geq 0 \\
& 0 \leq\left(x^{\text {max }}-x\right) \perp c_{b} \geq 0
\end{aligned}
$$

Ou seja, (6.8) significa que:

$$
\begin{array}{lllll}
\text { se } \quad x=x^{\text {min }} & \Rightarrow & c_{a} \geq 0 \quad \text { e } \quad c_{b}=0 & \Rightarrow & c(x) \geq 0 ; \\
\text { se } \quad x^{\text {min }}<x<x^{\text {max }} & \Rightarrow & c_{a}=0 \quad \text { e } \quad c_{b}=0 & \Rightarrow & c(x)=0 ; \\
\text { se } x=x^{\max } & \Rightarrow & c_{a}=0 \text { e } \quad c_{b} \geq 0 & \Rightarrow & c(x) \leq 0 .
\end{array}
$$

Observe que (6.7) e (6.9) são equivalentes.

Portanto, (6.8) pode ser representado algebricamente da seguinte forma:

$$
\begin{aligned}
& c_{i}(x)=c_{a_{i}}-c_{b_{i}} \\
& \left(x_{i}-x_{i}^{\text {min }}\right) c_{a_{i}}=0 \\
& \left(x_{i}^{\text {max }}-x_{i}\right) c_{b_{i}}=0 \\
& x_{i}-x_{i}^{\text {min }} \geq 0 \\
& x_{i}^{\text {max }}-x_{i} \geq 0 \\
& c_{a_{i}}, c_{b_{i}} \geq 0
\end{aligned}
$$

para $i=1, \cdots, n$. 


\subsection{Problemas de PNL com Restrições de Complementaridade}

Considere o seguinte problema de PNL com restrições de complementaridade:

$$
\begin{aligned}
& \min \quad f(x) \\
& \text { s.a: } \quad g_{i}(x)=0 \quad i=1, \cdots, p \\
& h_{i}(x) \leq 0 \quad i=1, \cdots, q \\
& x_{1_{i}}^{\text {min }} \leq x_{1_{i}} \leq x_{1_{i}}^{\max } \quad i=1, \cdots, m_{1} \\
& x_{2_{i}}^{\min } \leq x_{2_{i}} \leq x_{2_{i}}^{\max } \perp c_{i}(x) \quad i=1, \cdots, m_{2}
\end{aligned}
$$

onde $x_{1} \in \mathbb{R}^{m_{1}}$ e $x_{2} \in \mathbb{R}^{m_{2}}$, com $x=\left(x_{1}, x_{2}\right)$ e $x \in \mathbb{R}^{n} ; f: \mathbb{R}^{n} \rightarrow \mathbb{R} ; g: \mathbb{R}^{n} \rightarrow \mathbb{R}^{p}$, com $p<n ; h: \mathbb{R}^{n} \rightarrow \mathbb{R}^{q} ;$ e, finalmente, $c: \mathbb{R}^{n} \rightarrow \mathbb{R}^{m_{2}}$ representa o conjunto das funções que possuem uma relação de complementaridade com as variáveis $x_{2}$. Considere também que as funções $f, g, h$ e $c$ sejam de classe $C^{2}$.

Como demonstrado anteriormente, a restrição (6.11e) consiste em um problema de complementaridade, cujas variáveis possuem limites inferiores e superiores, e sua representação algébrica é dada por:

$$
\begin{aligned}
& c_{i}(x)=c_{a_{i}}-c_{b_{i}} \\
& \left(x_{2_{i}}-x_{2_{i}}^{\text {min }}\right) c_{a_{i}}=0 \\
& \left(x_{2_{i}}^{\max }-x_{2_{i}}\right) c_{b_{i}}=0 \\
& x_{2_{i}}-x_{2_{i}}^{\text {min }} \geq 0 \\
& x_{2_{i}}^{\text {max }}-x_{2_{i}} \geq 0 \\
& c_{a_{i}}, c_{b_{i}} \geq 0
\end{aligned}
$$


para $i=1, \cdots, m_{c}$ e onde $c_{a}, c_{b} \in \mathbb{R}^{m_{2}}$ são os vetores das variáveis auxiliares não negativas associadas, respectivamente, ao acréscimo e decréscimo de $c(x)$, ou seja:

$$
\begin{aligned}
& \text { se } \quad x_{2_{i}}=x_{2_{i}}^{\min } \quad \Rightarrow \quad c_{a_{i}} \geq 0 \quad \text { e } \quad c_{b_{i}}=0 \quad \Rightarrow \quad c_{i}(x) \geq 0 \\
& \text { se } \quad x_{2_{i}}^{\min }<x_{2_{i}}<x_{2_{i}}^{\max } \quad \Rightarrow \quad c_{a_{i}}=0 \quad \text { e } \quad c_{b_{i}}=0 \quad \Rightarrow \quad c_{i}(x)=0 \\
& \text { se } \quad x_{2_{i}}=x_{2_{i}}^{\max } \quad \Rightarrow \quad c_{a_{i}}=0 \quad \text { e } \quad c_{b_{i}} \geq 0 \quad \Rightarrow \quad c_{i}(x) \leq 0
\end{aligned}
$$

Dessa forma, substituindo (6.11e) por sua representação algébrica (6.12) e desmembrando as restrições canalizadas associadas aos limites das variáveis $x_{1}$ em restrições de desigualdade simples, obtém-se:

$$
\begin{aligned}
& \min \quad f(x) \\
& \text { s.a: } \quad g_{i}(x)=0 \quad i=1, \cdots, p \\
& h_{i}(x) \leq 0 \quad i=1, \cdots, q \\
& x_{1_{i}}^{\text {min }}-x_{1_{i}} \leq 0 \quad i=1, \cdots, m_{1} \\
& x_{1_{i}}-x_{1_{i}}^{\max } \leq 0 \quad i=1, \cdots, m_{1} \\
& c_{i}(x)=c_{a_{i}}-c_{b_{i}} \quad i=1, \cdots, m_{2} \\
& \left(x_{2_{i}}-x_{2_{i}}^{\min }\right) c_{a_{i}}=0 \quad i=1, \cdots, m_{2} \\
& \left(x_{2_{i}}^{\max }-x_{2_{i}}\right) c_{b_{i}}=0 \quad i=1, \cdots, m_{2} \\
& x_{2_{i}}-x_{2_{i}}^{\min } \geq 0 \quad i=1, \cdots, m_{2} \\
& x_{2_{i}}^{\max }-x_{2_{i}} \geq 0 \quad i=1, \cdots, m_{2} \\
& c_{a_{i}}, c_{b_{i}} \geq 0 \quad i=1, \cdots, m_{2}
\end{aligned}
$$

onde $s_{1} \in \mathbb{R}^{q}$ e $s_{2}, s_{3} \in \mathbb{R}^{n}$.

No entanto, métodos de otimização que empregam o cálculo de matrizes Hessianas no processo de resolução de problemas de PNL com restrições de complementaridade apresentam problemas numéricos em pontos de complementaridade não estrita, pois a 
matriz Hessiana da função Lagrangiana se torna singular nesses pontos (ROSEHART; ROMAN; SCHELLENBERG, 2005).

De acordo com as propostas na literatura, restrições de complementaridade são reformuladas pela função Fischer-Burmeister (PETOUSSIS; ZHANG; GODFREY, 2007; TODOROV, 2010; JIN; RYAN, 2011; ALMEIDA; SENNA, 2011), transformadas em restrições de desigualdade equivalentes (BENSON; SHANNO; VANDERBEI, 2002; RAGHUNATHAN; BIEGLER, 2005), ou tratadas por uma função penalidade (LEYFFER; LÓPEZ-CALVA; NOCEDAL, 2006). No entanto, nas reformulações pela função Fischer-Burmeister, métodos clássicos de PNL podem continuar apresentando problemas numéricos, pois as derivadas de primeira e segunda ordem dessa função tendem ao infinito em pontos de complementaridade não estrita; a abordagem pela função penalidade pode trazer problemas de mal condicionamento à matriz Hessiana à medida que o valor do parâmetro de penalidade cresça. Dessa forma, optou-se por reformular as restrições de complementaridade como restrições de desigualdade equivalentes.

As restrições (6.13g) e (6.13h) são reformuladas da seguinte forma:

$$
\begin{array}{lll}
\left(x_{2_{i}}-x_{2_{i}}^{\min }\right) c_{a_{i}}=0 & \Rightarrow & \left(x_{2_{i}}-x_{2_{i}}^{\min }\right) c_{a_{i}} \leq 0 \\
\left(x_{2_{i}}^{\max }-x_{2_{i}}\right) c_{b_{i}}=0 & \Rightarrow & \left(x_{2_{i}}^{\max }-x_{2_{i}}\right) c_{b_{i}} \leq 0
\end{array}
$$

para $i=1, \cdots, m_{2}$.

Devido aos limites de $x_{2}$ e às condições de não negatividade das variáveis auxiliares $c_{a}$ e $c_{b}$, as restrições $(6.14 \mathrm{a})$ e $(6.14 \mathrm{~b})$ só podem ser satisfeitas se $x_{2}$ for igual a um de seus limites e $c_{a}$ ou $c_{b}$ for igual a zero, satisfazendo a condição de complementaridade original.

Por fim, agrupando as restrições associadas aos limites das variáveis $x_{1}$ e $x_{2}$ e transformando as restrições de desigualdade em igualdades através da adição de variáveis de folga $s$ não negativas, o problema de PNL equivalente ao problema de PNL com 
restrições de complementaridade (6.11) é dado por:

$$
\begin{aligned}
& \min \quad f(x) \\
& \text { s.a: } \quad g_{i}(x)=0 \\
& i=1, \cdots, p \\
& h_{i}(x)+s_{1_{i}}=0 \\
& i=1, \cdots, q \\
& x_{i}^{\text {min }}-x_{i}+s_{2_{i}}=0 \\
& i=1, \cdots, n \\
& x_{i}-x_{i}^{\max }+s_{3_{i}}=0 \\
& i=1, \cdots, n \\
& c_{i}(x)=c_{a_{i}}-c_{b_{i}} \\
& i=1, \cdots, m_{2} \\
& \left(x_{2_{i}}-x_{2_{i}}^{\min }\right) c_{a_{i}}+s_{4_{i}}=0 \\
& i=1, \cdots, m_{2} \\
& \left(x_{2_{i}}^{\max }-x_{2_{i}}\right) c_{b_{i}}+s_{5_{i}}=0 \\
& i=1, \cdots, m_{2} \\
& s_{1_{i}} \geq 0 \\
& i=1, \cdots, q \\
& s_{2_{i}}, s_{3_{i}} \geq 0 \\
& i=1, \cdots, n \\
& s_{4_{i}}, s_{5_{i}} \geq 0 \\
& i=1, \cdots, m_{2} \\
& c_{a_{i}}, c_{b_{i}} \geq 0
\end{aligned}
$$

onde $s_{4}, s_{5} \in \mathbb{R}^{m_{2}}$.

\subsection{Exemplo Numérico}

Para exemplificar o uso do método da FLBMP na resolução de problemas de PNL com restrições de complementaridade, considere o seguinte problema, adaptado de Bazaraa (1993) e Baptista (2001):

$$
\begin{array}{ll}
\min & \left(x_{1}-2\right)^{4}+\left(x_{1}-2 x_{2}\right)^{2} \\
\text { s.a: } & x_{1}+x_{2}=3 \\
& x_{1}^{2}-x_{2} \leq 0 \\
& 1,5 \leq x_{2} \leq 2 \perp\left(x_{1}^{2}-x_{2}+0,4\right)
\end{array}
$$


A restrição (6.16d) consiste em um problema de complementaridade do tipo (6.6), e sua representação algébrica é dada por:

$$
\begin{aligned}
& x_{1}^{2}-x_{2}+0,4=c_{a}-c_{b} \\
& \left(x_{2}-1,5\right) c_{a}=0 \\
& \left(2-x_{2}\right) c_{b}=0 \\
& x_{2}-1,5 \geq 0 \\
& 2-x_{2} \geq 0 \\
& c_{a}, c_{b} \geq 0
\end{aligned}
$$

onde $c_{a}$ e $c_{b}$ são variáveis auxiliares não negativas associadas, respectivamente, ao acréscimo e decréscimo da função $x_{1}^{2}-x_{2}+0,4$.

Substituindo (6.16d) por sua representação algébrica, transformando as restrições de complementaridade em desigualdades equivalentes como em (6.14a) e (6.14b) e adicionando variáveis de folga $s$ não negativas para transformar as restrições de desigualdade em igualdades, obtém-se o seguinte problema modificado:

$$
\begin{array}{ll}
\min & \left(x_{1}-2\right)^{4}+\left(x_{1}-2 x_{2}\right)^{2} \\
\text { s.a: } & x_{1}+x_{2}-3=0 \\
& x_{1}^{2}-x_{2}+s_{1}=0 \\
& 1,5-x_{2}+s_{2}=0 \\
& x_{2}-2+s_{3}=0 \\
& x_{1}^{2}-x_{2}+0,4-c_{a}+c_{b}=0 \\
& \left(x_{2}-1,5\right) c_{a}+s_{4}=0 \\
& \left(2-x_{2}\right) c_{b}+s_{5}=0 \\
& s_{i} \geq 0 \\
& c_{a}, c_{b} \geq 0
\end{array} \quad i=1, \cdots, 5
$$


A FLBMP associada ao problema (6.18) é dada por:

$$
\begin{aligned}
\mathcal{L}(x, s, c, \lambda, \pi)= & \left(x_{1}-2\right)^{4}+\left(x_{1}-2 x_{2}\right)^{2}-\mu\left[\sigma_{1} \phi\left(s_{1}\right)+\sigma_{2} \phi\left(s_{2}\right)+\sigma_{3} \phi\left(s_{3}\right)+\right. \\
& \left.\sigma_{4} \phi\left(s_{4}\right)+\sigma_{5} \phi\left(s_{5}\right)+\sigma_{c_{a}} \phi\left(c_{a}\right)+\sigma_{c_{b}} \phi\left(c_{b}\right)\right]+ \\
& \lambda_{1}\left(x_{1}+x_{2}-3\right)+\pi_{1}\left(x_{1}^{2}-x_{2}+s_{1}\right)+ \\
& \pi_{2}\left(1,5-x_{2}+s_{2}\right)+\pi_{3}\left(x_{2}-2+s_{3}\right)+ \\
& \varphi_{1}\left(x_{1}^{2}-x_{2}+0,4-c_{a}+c_{b}\right)+ \\
& \varphi_{2}\left[\left(x_{2}-1,5\right) c_{a}+s_{4}\right]+\varphi_{3}\left[\left(2-x_{2}\right) c_{b}+s_{5}\right]
\end{aligned}
$$

Para resolver esse problema, o método da FLBMP foi inicializado com o ponto $x_{1}=1,05$ e $x_{2}=1,6$. As variáveis de folga $s_{i}$, para $i=1, \cdots, 5$, foram inicializadas, respectivamente, a partir das restrições $(6.18 \mathrm{c}),(6.18 \mathrm{~d}),(6.18 \mathrm{e}),(6.18 \mathrm{~g})$ e $(6.18 \mathrm{~h})$. O valor inicial dos multiplicadores de Lagrange $\lambda, \pi$ e $\sigma$ foram, respectivamente, 0,1 e 1 . Neste teste, considerou-se $\mu^{0}=10^{-1}$ e as tolerâncias para convergência foram $\xi_{1}=10^{-5}$, $\xi_{2}=10^{-4}$ e $\xi_{3}=10^{-5}$.

O processo de convergência do método da FLBMP é apresentado nas Tabelas 6.1, 6.2, 6.3 e 6.4. A representação gráfica do processo de convergência é dada na Figura 6.3. 
Tabela 6.1 - Convergência do método da FLBMP aplicado ao problema (6.16) - Função objetivo, variáveis $x$ e parâmetro $\mu$.

\begin{tabular}{ccccc}
\hline Iteração & F. Obj. & $x_{1}$ & $x_{2}$ & $\mu$ \\
\hline 0 & 5,9461 & 1,0500 & 1,6000 & $10^{-1}$ \\
1 & 4,3405 & 1,3226 & 1,6774 & $10^{-1}$ \\
2 & 6,1232 & 1,2028 & 1,7972 & $10^{-2}$ \\
3 & 6,3665 & 1,1881 & 1,8119 & $10^{-3}$ \\
4 & 6,3429 & 1,1895 & 1,8105 & $10^{-4}$ \\
5 & 6,3656 & 1,1882 & 1,8118 & $10^{-5}$ \\
\hline
\end{tabular}

Tabela 6.2 - Convergência do método da FLBMP aplicado ao problema (6.16) - Variáveis de folga $s$ e variáveis $c_{a}$ e $c_{b}$.

\begin{tabular}{cccccccc}
\hline Iteração & $s_{1}$ & $s_{2}$ & $s_{3}$ & $s_{4}$ & $s_{5}$ & $c_{a}$ & $c_{b}$ \\
\hline 0 & 0,4975 & 0,1000 & 0,4000 & 0,0000 & 0,0000 & 0,0000 & 0,0000 \\
1 & $-0,0719$ & 0,1774 & 0,3226 & $-0,0819$ & 0,0033 & 0,4616 & $-0,0102$ \\
2 & 0,3504 & 0,2972 & 0,2028 & $-0,0110$ & 0,0025 & 0,0371 & $-0,0125$ \\
3 & 0,4002 & 0,3119 & 0,1881 & 0,0001 & $-0,0000$ & $-0,0002$ & 0,0000 \\
4 & 0,3954 & 0,3105 & 0,1895 & $-0,0011$ & 0,0002 & 0,0035 & $-0,0011$ \\
5 & 0,4000 & 0,3118 & 0,1882 & 0,0000 & $-0,0000$ & $-0,0000$ & 0,0000 \\
\hline
\end{tabular}


Tabela 6.3 - Convergência do método da FLBMP aplicado ao problema (6.16) Multiplicadores de Lagrange $\lambda, \pi$ e $\varphi$.

\begin{tabular}{cccccccc}
\hline Iteração & $\lambda$ & $\pi_{1}$ & $\pi_{2}$ & $\pi_{3}$ & $\varphi_{1}$ & $\varphi_{2}$ & $\varphi_{3}$ \\
\hline 0 & 0,0000 & 1,0000 & 1,0000 & 1,0000 & 0,0000 & 0,0000 & 0,0000 \\
1 & $-6,2101$ & 3,5526 & 0,3605 & 0,2366 & 0,8017 & 5,5228 & 0,9680 \\
2 & $-5,1985$ & 0,0099 & 0,0012 & 0,0011 & 4,9817 & 16,7764 & 0,0772 \\
3 & $-4,7781$ & 0,0000 & 0,0000 & 0,0000 & 4,9614 & 15,9093 & 0,0008 \\
4 & $-4,8174$ & 0,0000 & 0,0000 & 0,0000 & 4,9638 & 15,9891 & 0,0000 \\
5 & $-4,7800$ & 0,0000 & 0,0000 & 0,0000 & 4,9617 & 15,9127 & 0,0000 \\
\hline
\end{tabular}

Tabela 6.4 - Convergência do método da FLBMP aplicado ao problema (6.16) Multiplicadores de Lagrange $\sigma$.

\begin{tabular}{cccccccc}
\hline Iteração & $\sigma_{1}$ & $\sigma_{2}$ & $\sigma_{3}$ & $\sigma_{4}$ & $\sigma_{5}$ & $\sigma_{c_{a}}$ & $\sigma_{c_{b}}$ \\
\hline 0 & 1,0000 & 1,0000 & 1,0000 & 1,0000 & 1,0000 & 1,0000 & 1,0000 \\
1 & 1,0000 & 1,0000 & 1,0000 & 1,0000 & 1,0000 & 1,0000 & 1,0000 \\
2 & 0,3553 & 0,0360 & 0,0237 & 0,5523 & 0,0968 & 0,0178 & 0,1114 \\
3 & 0,0001 & 0,0000 & 0,0000 & 16,7764 & 0,0008 & 0,0000 & 4,9973 \\
4 & 0,0000 & 0,0000 & 0,0000 & 0,0159 & 0,0000 & 0,0000 & 0,0050 \\
5 & 0,0000 & 0,0000 & 0,0000 & 15,9891 & 0,0000 & 0,0000 & 4,9638 \\
\hline
\end{tabular}




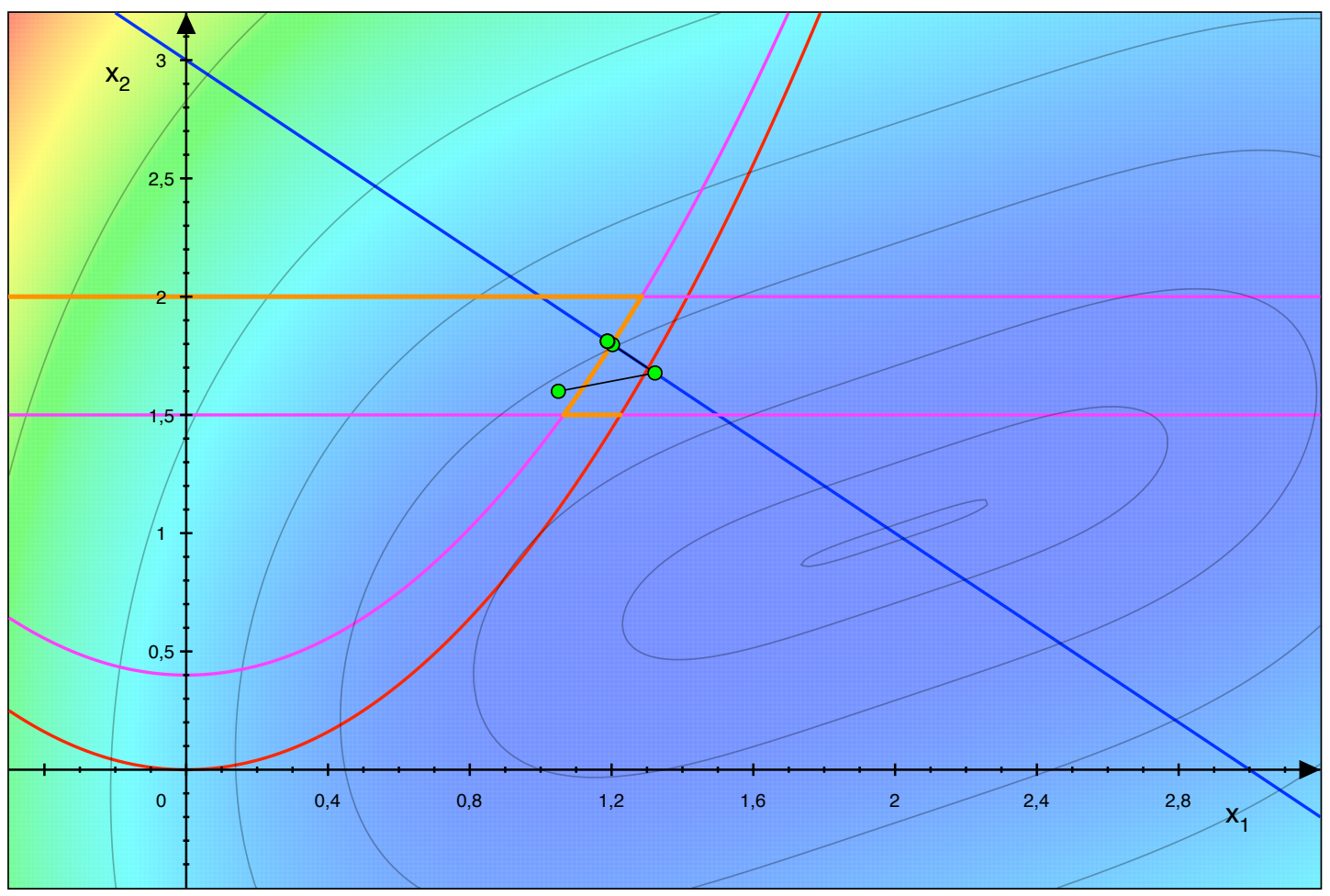

Figura 6.3 - Convergência do método da FLBMP aplicado ao problema (6.16). 


\section{Capítulo 7}

\section{Modelagem Estática de Sistemas de Energia Elétrica}

Neste capítulo, são apresentadas a modelagem estática de um SEE e a formulação básica do problema de FC, a partir do qual determina-se o estado da rede elétrica em função dos ajustes dos controles do sistema.

\subsection{Modelagem Estática de Sistemas de Energia Elétrica}

SEEs são tipicamente modelados por equações algébrico-diferenciais, que constituem uma classe de sistemas não lineares, da seguinte forma:

$$
\left[\begin{array}{l}
\dot{x} \\
0
\end{array}\right]=\left[\begin{array}{l}
f(x, y, \lambda, p) \\
g(x, y, \lambda, p)
\end{array}\right]=F(z, \lambda, p)
$$

onde $x \in \mathbb{R}^{n_{x}}$ é o vetor das variáveis que representam os estados dinâmicos dos geradores, dos controles e das cargas do sistema; $y \in \mathbb{R}^{n_{y}}$ é o vetor das variáveis dependentes que representam as magnitudes e ângulos de fase das tensões nas barras de carga; $\lambda \in \mathbb{R}_{+}$ é um parâmetro de variação lenta e "incontrolável" usado para representar mudanças 
(acréscimo) na geração e na carga; e $p \in \mathbb{R}^{n_{p}}$ é o vetor dos parâmetros "controláveis" do sistema, como as injeções de potência ativa e reativa na rede, os taps de transformadores em-fase, as magnitudes de tensão especificadas para as barras de geração. A função $f: \mathbb{R}^{n_{x}} \times \mathbb{R}^{n_{y}} \times \mathbb{R}_{+} \times \mathbb{R}^{n_{p}} \rightarrow \mathbb{R}^{n_{x}}$, que constitui um campo vetorial não linear associado às variáveis de estado $x$, representa as equações diferenciais do modelo, como, por exemplo, as equações associadas à dinâmica dos geradores. A função $g: \mathbb{R}^{n_{x}} \times \mathbb{R}^{n_{y}} \times \mathbb{R}_{+} \times \mathbb{R}^{n_{p}} \rightarrow \mathbb{R}^{n_{y}}$, que constitui um campo vetorial não linear associado às variáveis dependentes $y$, representa as equações algébricas do modelo, como, por exemplo, as equações de balanço de potência ativa e reativa nas barras do sistema.

Em condições normais de operação, os pontos de equilíbrio estáveis $z_{o}=\left(x_{o}, y_{o}\right)$ do modelo de um SEE devem satisfazer à seguinte condição:

$$
F\left(z_{o}, \lambda_{o}, p_{o}\right)=\left[\begin{array}{l}
f\left(z_{o}, \lambda_{o}, p_{o}\right) \\
g\left(z_{o}, \lambda_{o}, p_{o}\right)
\end{array}\right]=0
$$

No entanto, os pontos de equilíbrio para condições normais de operação são obtidos, na prática, a partir das soluções do seguinte subconjunto de (7.2) (MUÑOZ, 2008):

$$
G\left(\hat{z}_{o}, \lambda_{o}, \hat{p}_{o}\right)=\left.G\right|_{o}=0 \quad \subset \quad F\left(z_{o}, \lambda_{o}, p_{o}\right)=\left.F\right|_{o}=0
$$

onde $\left.G\right|_{o}=0$ representa o conjunto das equações de balanço de potência ativa e reativa nas barras do sistema; $G \subseteq g ; \hat{z}_{o} \in \mathbb{R}^{n_{\hat{z}}} \subseteq z_{o}$ é o conjunto das magnitudes e ângulos de fase das tensões nas barras do sistema; e $\hat{p}_{o} \in \mathbb{R}^{n_{\hat{p}}} \subseteq p_{o}$ representa as magnitudes de tensão especificadas (desejadas) para as barras de geração, as injeções de potência ativa nas barras de geração, as injeções de potência ativa e reativa nas barras de carga, os valores dos taps de transformadores em-fase etc.

É importante ressaltar que as soluções do conjunto de equações $G$ em (7.3) não correspondem necessariamente aos pontos de equilíbrio do sistema (7.1), uma vez que a solução $\left.G\right|_{o}=0$ não implica em $\left.F\right|_{o}=0$. Na prática, o aspecto dinâmico da modelagem 
de um SEE pode ser ignorado em situações nas quais as mudanças no sistema em função do tempo são suficientemente lentas para que se possa desconsiderar os efeitos transitórios (hipótese de operação quasi-estática) (MONTICELLI, 1983).

Dessa forma, passa-se agora à formulação básica do problema de FC, a partir do qual determina-se o estado da rede elétrica, em regime permanente, em função dos ajustes dos controles do sistema.

\subsection{Formulação do Problema de Fluxo de Carga}

O cálculo de FC, ou fluxo de potência, consiste na determinação de grandezas que representam o comportamento de uma rede elétrica em regime permanente. Basicamente, a resolução desse problema é realizada através de métodos específicos para a resolução do sistema de equações não lineares que representa o modelo da rede elétrica, fornecendo seu

estado, isto é, a magnitude e o ângulo de fase da tensão em cada barra da rede. Com o estado conhecido, calculam-se outras grandezas de interesse, como: a geração de potência reativa nas barras de geração, a distribuição dos fluxos de potência ativa e reativa, as perdas de potência ativa nas linhas de transmissão etc.

O problema de FC pode ser formulado por um sistema de equações e inequações algébricas não lineares que correspondem, respectivamente, às leis de Kirchhoff e a um conjunto de restrições operacionais da rede elétrica e seus componentes. Na formulação básica do problema, a cada barra $k$ da rede são associadas quatro grandezas escalares:

- a magnitude $V_{k}$ da tensão na barra;

- o ângulo de fase $\theta_{k}$ da tensão na barra;

- a potência ativa líquida $P_{k}$ injetada na barra;

- e a potência reativa líquida $Q_{k}$ injetada na barra. 
Na formulação básica do problema de FC, somente duas dessas grandezas em cada barra são conhecidas a priori. Dependendo de quais grandezas nodais são consideradas dados e quais são consideradas incógnitas, definem-se três tipos de barras:

1. Barra $V \theta$, barra de referência angular, ou barra slack:

- $V_{k}$ e $\theta_{k}^{1}$ são dados;

- e $P_{k}$ e $Q_{k}$ são calculadas de forma a fechar o balanço de potência do sistema.

2. Barra $P V$, ou barra de geração:

- $P_{k}$ e $V_{k}$ são dadas;

- e $Q_{k}$ e $\theta_{k}$ são calculados.

3. Barra $P Q$, ou barra de carga:

- $P_{k}$ e $Q_{k}$ são dadas;

- e $V_{k}$ e $\theta_{k}$ são calculados.

Na determinação do conjunto de equações não lineares que representa o modelo da rede elétrica, duas equações são associadas a cada barra, obtidas através da lei dos nós de Kirchhoff, segundo a qual as injeções de potência ativa e reativa em uma barra $k$ são iguais, respectivamente, à soma dos fluxos de potência ativa e reativa que deixam essa barra. A imposição da conservação das potências ativa e reativa em cada barra da rede pode ser expressa matematicamente por:

$$
\begin{array}{ll}
P_{k}-\sum_{m \in \mathcal{V}_{k}} P_{k m}\left(V_{k}, V_{m}, \theta_{k}, \theta_{m}, t_{k m}\right)=0 & \forall k \in \mathcal{B} \\
Q_{k}+Q_{k}^{s h}\left(V_{k}, b_{k}^{s h}\right)-\sum_{m \in \mathcal{V}_{k}} Q_{k m}\left(V_{k}, V_{m}, \theta_{k}, \theta_{m}, t_{k m}\right)=0 & \forall k \in \mathcal{B}
\end{array}
$$

\footnotetext{
${ }^{1} \theta_{k}$ é considerado como referência angular da rede elétrica e é geralmente fixado em $0^{\circ}$. Uma mesma distribuição de fluxos de potência pode ser obtida se for somada uma constante arbitrária a todos os ângulos de fase das tensões do sistema elétrico, ou seja, o problema de FC é indeterminado nas variáveis $\theta$, o que torna necessária a adoção de uma referência angular.
} 
onde:

- $\mathcal{B}$ é o conjunto de todas as barras do sistema elétrico;

- $\mathcal{V}_{k}$ é conjunto das barras vizinhas à barra $k$;

- $V_{k}$ e $V_{m}$ são as magnitudes das tensões nas barras terminais do ramo $k$ - $m$;

- $\theta_{k}$ e $\theta_{m}$ são os ângulos de fase das tensões nas barras terminais do ramo $k-m$;

- se o ramo $k-m$ for um transformador em-fase, $t_{k m}$ é o tap desse transformador;

- $P_{k m}$ é o fluxo de potência ativa no ramo $k$ - $m$, definido em função dos parâmetros do ramo e das tensões nas barras $k$ e $m$;

- $Q_{k m}$ é o fluxo de potência reativa no ramo $k$ - $m$, definido em função dos parâmetros do ramo e das tensões nas barras $k$ e $m$;

- e $Q_{k}^{s h}$ é a componente da injeção de potência reativa na barra $k$ pelo elemento shunt, sendo $b_{k}^{s h}$ a susceptância do elemento shunt.

As equações (7.4) e (7.5) são denominadas, respectivamente, equações de balanço de potência ativa e reativa ${ }^{2}$.

Além das equações de balanço de potência, uma rede elétrica também possui uma série de limites técnico-operacionais que influem diretamente na determinação das suas condições de operação e que devem, portanto, ser incluídos na sua modelagem para que o estado da rede possa ser determinado corretamente. Na resolução do problema de FC, esses limites operacionais da rede elétrica e de seus componentes são, geralmente, verificados por "passos" externos ao algoritmo de resolução das equações de balanço do modelo da rede (MONTICELLI, 1983).

\footnotetext{
${ }^{2}$ As equações de balanço de potência, assim como as deduções das expressões gerais dos fluxos e injeções de potência ativa e reativa nas barras da rede elétrica, são apresentadas no Apêndice A.
} 
O conjunto de inequações que fazem parte do problema de FC é formado por:

- limites da geração de potência reativa nas barras com controle de reativos:

$$
Q_{G_{k}}^{\min } \leq Q_{G_{k}}(V, \theta) \leq Q_{G_{k}}^{\max } \quad \forall k \in \mathcal{G}
$$

- limites das magnitudes de tensão das barras de carga:

$$
V_{k}^{\min } \leq V_{k} \leq V_{k}^{\max } \quad \forall k \in \mathcal{C}
$$

- limites dos taps de transformadores em-fase:

$$
t_{k m}^{\min } \leq t_{k m} \leq t_{k m}^{\max } \quad \forall k, m \in \mathcal{T}
$$

onde $\mathcal{T}$ é o conjunto dos ramos $k-m$ que representam transformadores em-fase com tap controlável.

\subsubsection{Resolução do Problema de Fluxo de Carga}

Com o estado da rede conhecido, calculam-se as outras grandezas de interesse, isto é, $P_{k}$ e $Q_{k}$ na barra slack, $Q_{k}$ nas barras $P V$, a distribuição dos fluxos de potência ativa e reativa nas linhas de transmissão e transformadores etc. Sendo assim, o sistema de equações (7.4)-(7.5) é decomposto em dois subsistemas de equações não lineares, de forma a se determinar, primeiramente, o estado $\left(V_{k}, \theta_{k}\right)$ da rede e, em seguida, as demais grandezas de interesse.

Seja $n_{p v}$ o número de barras $P V, n_{p q}$ o número de barras $P Q, \mathcal{G}$ o conjunto das barras de geração, $\mathcal{G}^{\prime}$ o conjunto das barras de geração menos a barra slack, e $\mathcal{C}$ o conjunto das barras de carga da rede elétrica. A divisão do sistema de equações (7.4)-(7.5) em dois subsistemas é feita da seguinte forma:

- Subsistema 1:

Neste subproblema são dadas $P_{k}$ e $V_{k}$ nas barras $P V$, e $P_{k}$ e $Q_{k}$ nas barras $P Q$; e pretende-se calcular $\theta_{k}$ nas barras $P V$ e $V_{k}$ e $\theta_{k}$ nas barras $P Q$. Trata-se de um 
sistema de $2 n_{p q}+n_{p v}$ equações não lineares com o mesmo número de incógnitas. O conjunto de equações deste subsistema é dado por:

$$
\begin{array}{ll}
P_{k}-\sum_{m \in \mathcal{V}_{k}} P_{k m}\left(V_{k}, V_{m}, \theta_{k}, \theta_{m}, t_{k m}\right)=0 & \forall k \in \mathcal{G}^{\prime} \cup \mathcal{C} \\
Q_{k}+Q_{k}^{s h}\left(V_{k}, b_{k}^{s h}\right)-\sum_{m \in \mathcal{V}_{k}} Q_{k m}\left(V_{k}, V_{m}, \theta_{k}, \theta_{m}, t_{k m}\right)=0 & \forall k \in \mathcal{C}
\end{array}
$$

- Subsistema 2:

Após a resolução do subsistema 1 e, portanto, sendo conhecido o estado $\left(V_{k}, \theta_{k}\right)$ em todas as barras, deseja-se calcular $P_{k}$ e $Q_{k}$ na barra slack e $Q_{k}$ nas barras $P V$. Trata-se de um sistema com $n_{p v}+2$ equações não lineares com o mesmo número de incógnitas. O conjunto de equações deste subsistema é dado por:

$$
\begin{array}{ll}
P_{k}-\sum_{m \in \mathcal{V}_{k}} P_{k m}\left(V_{k}, V_{m}, \theta_{k}, \theta_{m}, t_{k m}\right)=0 & \forall k \in V \theta \\
Q_{k}+Q_{k}^{s h}\left(V_{k}, b_{k}^{s h}\right)-\sum_{m \in \mathcal{V}_{k}} Q_{k m}\left(V_{k}, V_{m}, \theta_{k}, \theta_{m}, t_{k m}\right)=0 & \forall k \in \mathcal{G}
\end{array}
$$

Como as variáveis do subsistema 1 são implícitas, torna-se necessário o uso de métodos iterativos para sua resolução, dentre os quais destacam-se os métodos de Gauss-Seidel, de Newton-Raphson (TINNEY; HART, 1967), de Newton desacoplado (STOTT, 1972), o desacoplado rápido (STOTT; ALSAÇ, 1974), entre outros. As variáveis do subsistema 2 aparecem de forma explícita, o que torna trivial seu processo de resolução.

A solução do subsistema 1 é alcançada quando a norma dos erros das equações de balanço for menor que uma tolerância predeterminada. Quando uma solução do problema de FC não apresenta resultados aceitáveis, são realizados ajustes nas variáveis associadas aos dispositivos de controle da rede elétrica e o sistema de equações não lineares do problema de FC é resolvido novamente. Por exemplo, se a solução do problema apresentar 
um valor de magnitude de tensão em uma barra que esteja fora dos limites operacionais, uma compensação por injeção de reativos ou uma mudança da posição do tap de um transformador em-fase podem ser usadas para corrigir o problema.

O controle de magnitude de tensão por injeção de reativos ou pelo ajuste do tap de transformadores em-fase trazem algumas complicações adicionais ao processo de resolução do problema de FC. A convergência do processo iterativo fica, geralmente, mais lenta; a interferência entre controles que são eletricamente próximos pode levar, em algumas situações, à não convergência do processo iterativo; e, além desses fatores, a ocorrência de soluções múltiplas para um mesmo problema torna-se bastante frequente quando esses dispositivos de controle são incluídos na modelagem do sistema (MONTICELLI, 1983).

Através de operadores experientes, o FC pode ser empregado como um instrumento de ajuste das variáveis de controle da rede para situações localizadas. Quando uma grande quantidade de variáveis de controle precisar ser ajustada, o uso do FC torna-se um exaustivo processo de tentativas e erros. Nesses casos, metodologias baseadas na resolução de problemas de FPO são a melhor alternativa, ajustando simultaneamente, e de maneira ótima, todas as variáveis de controle do sistema em função de um determinado desempenho operacional (RAMOS et al., 1995).

Para que a atuação de dispositivos de controle de tensão como taps de transformadores em-fase, bancos de capacitores e reatores shunt possa ser representada explicitamente em formulações de problemas de FPO, passa-se, agora, à sua modelagem por problemas de complementaridade. 


\section{Capítulo 8}

\section{Modelagem do Controle de Tensão por Problemas de Complementaridade}

Um SEE possui uma série de dispositivos de controle que influenciam diretamente nas suas condições de operação. Na formulação de um problema de FPOR, os controles de tensão da rede elétrica são passíveis de ajustes para que um desempenho operacional ótimo da rede elétrica seja alcançado. Porém, para que a aplicação desses problemas à operação de SEEs seja viável, torna-se necessário restringir, sempre que possível, os ajustes em algumas de suas variáveis de controle, pois um número muito grande de ajustes é praticamente impossível de ser realizado antes que o estado da rede mude significativamente.

A exemplo da modelagem proposta por Rosehart, Roman e Schellenberg (2005) para o controle da magnitude de tensão por geração de reativos, este trabalho propõe a representação da atuação de dispositivos de controle de tensão como transformadores em-fase, bancos de capacitores e reatores shunt por problemas de complementaridade, os quais modelam algebricamente a condição de que esses dispositivos atuem somente quando um dos limites de tensão da barra controlada por eles for atingido. Dessa forma, restringe-se o número de ajustes nos taps dos transformadores em-fase e de chaveamentos de bancos de capacitores e reatores shunt recomendados pela resolução do FPOR. 


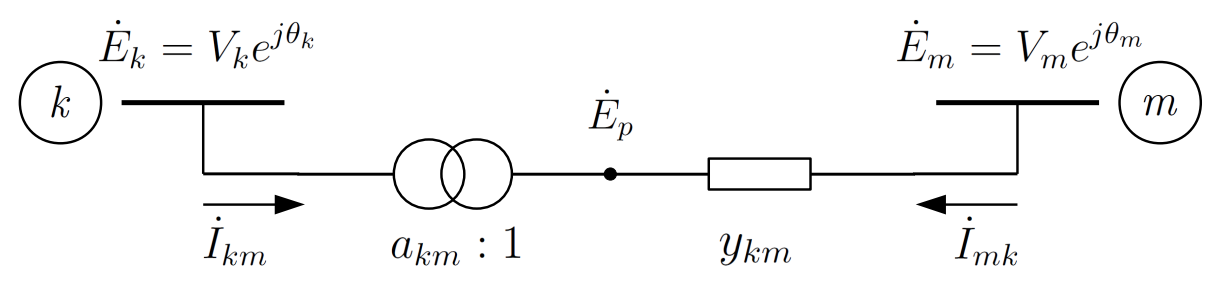

Figura 8.1 - Modelo de um transformador em-fase.

Neste capítulo, os controles da magnitude de tensão por ajustes nos taps de transformadores em-fase e chaveamentos de bancos de capacitores e reatores shunt são modelados através de problemas de complementaridade.

\subsection{Modelagem da Atuação de Transformadores Em-fase}

Para que o controle da magnitude de tensão por transformadores em-fase possa ser modelado por problemas de complementaridade, deve-se, primeiramente, conhecer a forma de operação desse dispositivo de controle na rede de transmissão. Para isso, considere a representação geral de transformadores (em-fase e defasadores) dada na Figura 8.1, que consiste basicamente em um auto-transformador ideal com relação de transformação $a_{k m}: 1$ e uma admitância série $y_{k m}$. Para transformadores em-fase, $a_{k m}=t_{k m}$.

Na Figura 8.1, as equações de $\dot{I}_{k m}$ e $\dot{I}_{m k}$ são determinadas a partir das relações entre as magnitudes das tensões nos nós $k$ e $p$, do fato do transformador $k-p$ ser ideal, e da aplicação da lei das tensões de Kirchhoff à análise desse circuito ${ }^{1}$. Dessa forma, as equações das correntes $\dot{I}_{k m}$ e $\dot{I}_{m k}$ definidas em função das tensões fasoriais nas barras $k$ e

\footnotetext{
${ }^{1}$ Para maiores informações, consulte o Apêndice A.
} 


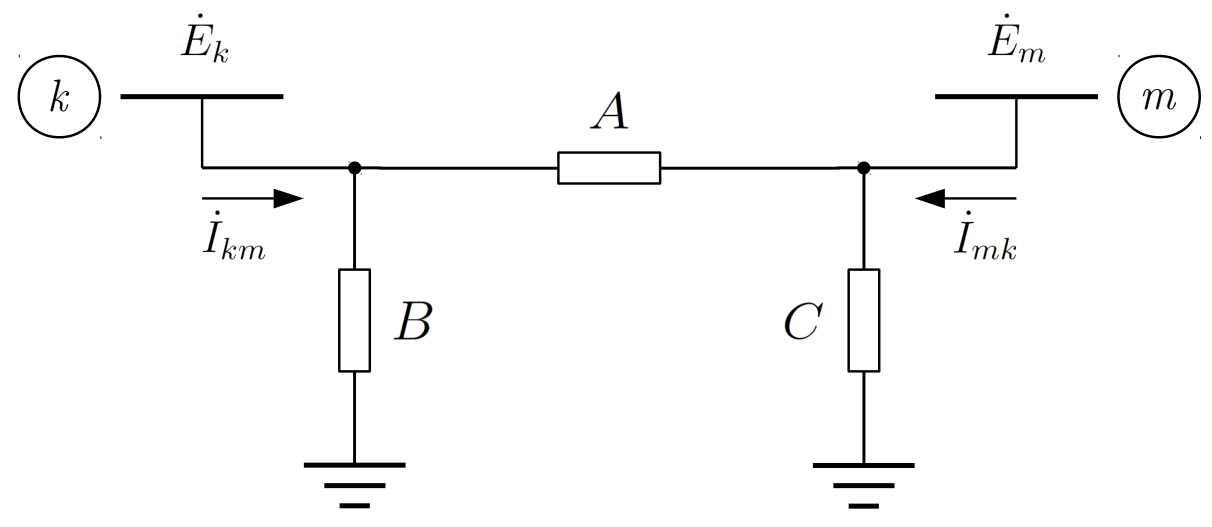

Figura 8.2 - Circuito equivalente tipo $\pi$ de transformadores em-fase.

$m$ são dadas por:

$$
\begin{aligned}
& \dot{I}_{k m}=\left(\frac{1}{t_{k m}^{2}} y_{k m}\right) \dot{E}_{k}+\left(-\frac{1}{t_{k m}} y_{k m}\right) \dot{E}_{m} \\
& \dot{I}_{m k}=\left(-\frac{1}{t_{k m}} y_{k m}\right) \dot{E}_{k}+\left(y_{k m}\right) \dot{E}_{m}
\end{aligned}
$$

O transformador em-fase também pode ser representado por um circuito equivalente do tipo $\pi$, conforme é ilustrado na Figura 8.2. A determinação das admitâncias $A, B$ e $C$ do circuito equivalente é feita pela comparação das expressões as correntes $\dot{I}_{k m}$ e $\dot{I}_{m k}$ do modelo da Figura 8.1 com as correntes correspondentes do circuito equivalente da Figura 8.2 (MONTICELLI, 1983).

Para o modelo $\pi$ da Figura 8.2 , as correntes $\dot{I}_{k m}$ e $\dot{I}_{m k}$ podem ser escritas da seguinte forma:

$$
\begin{aligned}
& \dot{I}_{k m}=(A+B) \dot{E}_{k}+(-A) \dot{E}_{m} \\
& \dot{I}_{m k}=(-A) \dot{E}_{k}+(A+C) \dot{E}_{m}
\end{aligned}
$$

A partir da identificação dos coeficientes de $\dot{E}_{k}$ e $\dot{E}_{m}$ nas expressões (8.1), (8.2), (8.3) e (8.4), obtém-se as seguintes expressões para as admitâncias $A, B$ e $C$ do circuito 
equivalente:

$$
\begin{aligned}
& A=\frac{1}{t_{k m}} y_{k m} \\
& B=\frac{1}{t_{k m}}\left(\frac{1}{t_{k m}}-1\right) y_{k m} \\
& C=\left(1-\frac{1}{t_{k m}}\right) y_{k m}
\end{aligned}
$$

As expressões (8.5) permitem a análise do efeito da relação de transformação $t_{k m}: 1$ sobre as magnitudes das tensões terminais nas barras $k$ e $m$. Para isso, considere inicialmente que $t_{k m}=1$. Neste caso, as admitâncias $B$ e $C$ são nulas, e o circuito equivalente $\pi$ reduz-se à admitância série $y_{k m}$. Alterando-se $t_{k m}$ para um valor maior que $1, B$ terá sinal contrário a $y_{k m}$ e, portanto, será do tipo capacitivo, enquanto $C$ será do tipo indutivo: isto implicará em uma tendência em aumentar a magnitude de tensão da barra $k$ e diminuir a da barra $m$. Ao contrário, quando $t_{k m}$ for menor que $1, B$ será do tipo indutivo (mesmo sinal que $y_{k m}$ ), enquanto $C$ será do tipo capacitivo: haverá uma tendência em diminuir a magnitude de tensão da barra $k$ e a aumentar a da barra $m$.

Para modelar da atuação desse dispositivo de controle, considere primeiramente o caso em que a barra cuja magnitude de tensão é controlada seja a barra $m$. A forma dos ajustes na variável $t_{k m}$ para este caso está diretamente ligada à análise da admitância $C$ do modelo equivalente $\pi$. Quando a magnitude de tensão na barra $m$ atinge seu limite mínimo, a variável $t_{k m}$ é ajustada de forma a aumentar a magnitude de tensão nessa barra, ou seja, diminui-se $t_{k m}$. Ao contrário, quando a magnitude de tensão na barra $m$ atinge seu limite máximo, a variável $t_{k m}$ é ajustada de forma a diminuir a magnitude de tensão nessa barra, ou seja, aumenta-se $t_{k m}$. 
Por essa análise, o controle da magnitude de tensão na barra $m$ por um transformador em-fase pode ser expresso da seguinte forma:

$$
\begin{array}{llll}
\text { se } \quad V_{m}=V_{m}^{\text {min }} & \Rightarrow & \Delta t_{k m}<0 ; \\
\text { se } \quad V_{m}^{\text {min }}<V_{m}<V_{m}^{\max } & \Rightarrow & \Delta t_{k m}=0 ; \\
\text { se } \quad V_{m}=V_{m}^{\max } & \Rightarrow & \Delta t_{k m}>0 .
\end{array}
$$

sendo $\Delta t_{k m}=t_{k m}-t_{k m_{o}}$ o ajuste na variável de controle $t_{k m}$, e $t_{k m_{o}}$ seu valor inicial. Observe que, nesta representação, a barra cuja tensão é controlada possui um intervalo de valores, e não um valor especificado, para a magnitude de tensão controlada. Nessas condições, a variável $t_{k m}$ será ajustada somente quando a magnitude de tensão na barra $m$ não puder ser mantida entre seus limites.

Comparando-se (8.6) com (6.7), o controle da magnitude de tensão na barra $m$ pelo ajuste na variável $t_{k m}$ associada ao tap do transformador em-fase do ramo $k-m$ pode ser modelado pelo seguinte problema de complementaridade:

$$
V_{m}^{\min } \leq V_{m} \leq V_{m}^{\max } \perp-\Delta t_{k m}
$$

Assim como (6.6) foi transformado na associação de dois problemas de complementaridade do tipo (6.4), o problema de complementaridade (8.7) também pode ser modificado da seguinte forma:

$$
\begin{aligned}
& -\Delta t_{k m}=t_{a_{k m}}-t_{b_{k m}} \\
& 0 \leq\left(V_{m}-V_{m}^{\text {min }}\right) \perp t_{a_{k m}} \geq 0 \\
& 0 \leq\left(V_{m}^{\text {max }}-V_{m}\right) \perp t_{b_{k m}} \geq 0
\end{aligned}
$$

onde $t_{a_{k m}}$ e $t_{b_{k m}}$ são variáveis auxiliares não negativas que podem, dependendo do valor da magnitude de tensão $V_{m}$, aumentar ou diminuir o ajuste na variável $t_{k m}$. 
Portanto, o controle da magnitude de tensão por transformadores em-fase pode ser modelado algebricamente da seguinte forma:

$$
\begin{aligned}
& \Delta t_{k m}=-t_{a_{k m}}+t_{b_{k m}} \\
& \left(V_{m}-V_{m}^{\text {min }}\right) t_{a_{k m}}=0 \\
& \left(V_{m}^{\text {max }}-V_{m}\right) t_{b_{k m}}=0 \\
& \left(V_{m}-V_{m}^{\text {min }}\right) \geq 0 \\
& \left(V_{m}^{\text {max }}-V_{m}\right) \geq 0 \\
& t_{a_{k m}} \geq 0 \\
& t_{b_{k m}} \geq 0
\end{aligned}
$$

Raciocínio análogo pode ser aplicado para o caso em que a barra cuja magnitude de tensão é controlada seja a barra $k$. A forma dos ajustes na variável $t_{k m}$ para este caso está diretamente ligada à análise da admitância $B$ do modelo equivalente $\pi$. Quando a magnitude de tensão na barra $k$ atinge seu limite mínimo, a variável $t_{k m}$ é ajustada de forma a aumentar a magnitude de tensão nessa barra, ou seja, aumenta-se $t_{k m}$. Ao contrário, quando a magnitude de tensão na barra $k$ atinge seu limite máximo, a variável $t_{k m}$ é ajustada de forma a diminuir a magnitude de tensão dessa barra, ou seja, diminui-se $t_{k m}$.

Por essa análise, o controle da magnitude de tensão na barra $k$ por um transformador em-fase pode ser expresso da seguinte forma:

$$
\begin{array}{llll}
\text { se } \quad V_{k}=V_{k}^{\text {min }} & \Rightarrow & \Delta t_{k m}>0 ; \\
\text { se } \quad V_{k}^{\text {min }}<V_{k}<V_{k}^{\text {max }} & \Rightarrow & \Delta t_{k m}=0 ; \\
\text { se } \quad V_{k}=V_{k}^{\text {max }} & \Rightarrow & \Delta t_{k m}<0 .
\end{array}
$$


e o problema de complementaridade formulado a partir delas é dado por:

$$
V_{k}^{\text {min }} \leq V_{k} \leq V_{k}^{\max } \perp \Delta t_{k m}
$$

O problema (8.11) modificado é dado por:

$$
\begin{aligned}
& \Delta t_{k m}=t_{a_{k m}}-t_{b_{k m}} \\
& 0 \leq\left(V_{k}-V_{k}^{\text {min }}\right) \perp t_{a_{k m}} \geq 0 \\
& 0 \leq\left(V_{k}^{\text {max }}-V_{k}\right) \perp t_{b_{k m}} \geq 0
\end{aligned}
$$

sendo sua representação algébrica dada por:

$$
\begin{aligned}
& \Delta t_{k m}=t_{a_{k m}}-t_{b_{k m}} \\
& \left(V_{k}-V_{k}^{\text {min }}\right) t_{a_{k m}}=0 \\
& \left(V_{k}^{\text {max }}-V_{k}\right) t_{b_{k m}}=0 \\
& \left(V_{k}-V_{k}^{\text {min }}\right) \geq 0 \\
& \left(V_{k}^{\text {max }}-V_{k}\right) \geq 0 \\
& t_{a_{k m}} \geq 0 \\
& t_{b_{k m}} \geq 0
\end{aligned}
$$

\subsection{Modelagem da Atuação de Bancos de Capacitores e Reatores Shunt}

Em muitos SEEs, as capacidades do controle reativo das máquinas síncronas não são suficientes para manter as magnitudes da tensão dentro dos limites predeterminados para todas as condições de carregamento. As condições de carga variadas do sistema durante as situações de carga baixa e de pico implicam que a potência reativa necessária 
para manter as magnitudes da tensão desejada varia significativamente. É impossível, e também imprudente, usar as capacidades do controle reativo das máquinas síncronas para compensar isso, visto que muitas máquinas síncronas trabalham próximo aos seus limites de capacidade, e o controle rápido e contínuo de potência reativa ofertado pela máquina síncrona não estará disponível. Portanto, na maioria dos sistemas, os bancos de capacitores chaveados por disjuntores e reatores shunt são usados para um controle de maior porte da potência reativa, de modo que os geradores síncronos podem ser usados para o controle rápido e contínuo (GÓMEZ-EXPÓSITO; CONEJO; CAÑIZARES, 2011).

Geralmente, capacitores shunt são chaveados durante condições de alto carregamento, quando o consumo de potência reativa pelas cargas e as reatâncias das linhas de transmissão são mais altas. Desde que essas variações de carga sejam lentas e previsíveis, nenhum controle rápido é necessário e os bancos de capacitores podem ser chaveados pelo disjuntor. Nos sistemas com linhas de transmissão de alta tensão longas, a geração de potência reativa dessas linhas pode ser muito alta durante condições de carga leve; para manter as tensões em níveis aceitáveis, reatores shunt são necessários (GÓMEZ-EXPÓSITO; CONEJO; CAÑIZARES, 2011).

Portanto, os capacitores e reatores shunt são, basicamente, uma forma de injetar e absorver potência reativa no ponto onde são instalados. Esses componentes são modelados como susceptâncias diretamente conectadas às barras, como pode ser visto na Figura 8.3. Se a susceptância $b_{k}^{s h}$ do shunt for positiva, esse shunt é do tipo capacitivo. Caso ela seja negativa, esse shunt é do tipo indutivo.

Para modelar a atuação desse dispositivo de controle, considere a seguinte expressão da injeção de potência reativa pelo shunt:

$$
Q_{k}^{s h}=b_{k}^{s h} V_{k}^{2}
$$

Quando a magnitude de tensão na barra $k$ atinge seu limite mínimo, $b_{k}^{s h}$ é ajustada de forma a aumentar a injeção, ou diminuir a absorção, de potência reativa nesse ponto, 


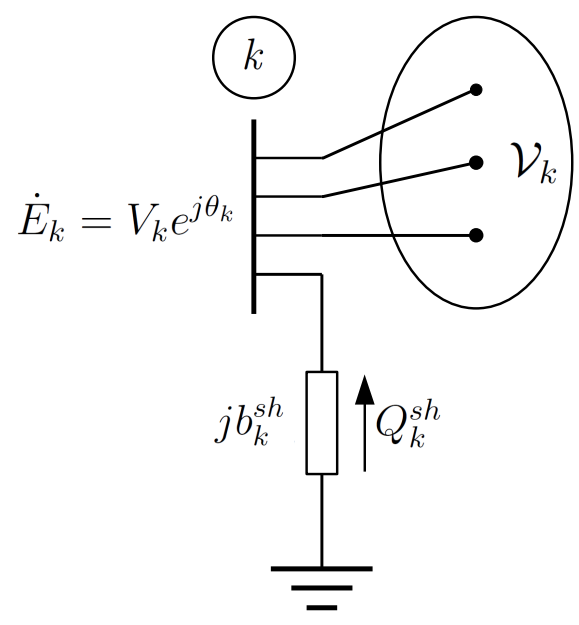

Figura 8.3 - Modelo de um shunt de barra.

ou seja, aumenta-se $b_{k}^{s h}$. Ao contrário, quando a magnitude de tensão na barra $m$ atinge seu limite máximo, $b_{k}^{s h}$ é ajustada de forma a diminuir a injeção, ou aumentar a absorção, de potência reativa nessa barra, ou seja, diminui-se $b_{k}^{s h}$.

Portanto, o controle da magnitude de tensão por elementos shunt pode ser expresso da seguinte forma:

$$
\begin{array}{llll}
\text { se } \quad V_{k}=V_{k}^{\text {min }} & \Rightarrow & \Delta b_{k}^{\text {sh }}>0 ; \\
\text { se } \quad V_{k}^{\text {min }}<V_{k}<V_{k}^{\text {max }} & \Rightarrow & \Delta b_{k}^{s h}=0 ; \\
\text { se } \quad V_{k}=V_{k}^{\text {max }} & \Rightarrow & \Delta b_{k}^{\text {sh }}<0 .
\end{array}
$$

sendo $\Delta b_{k}^{s h}=b_{k}^{s h}-b_{k_{o}}^{s h}$ o ajuste na variável de controle $b_{k}^{s h}$, e $b_{k_{o}}^{s h}$ seu valor inicial. Observe que nesta representação a barra cuja tensão é controlada também possui um intervalo de valores, e não um valor especificado, para a magnitude de tensão controlada. Nessas condições, a variável $b_{k}^{s h}$ deverá ser ajustada somente quando a magnitude de tensão na barra $k$ não puder ser mantida entre seus limites.

Comparando-se (8.15) com (6.7), o controle da magnitude de tensão na barra $k$ pelo ajuste na variável $b_{k}^{s h}$ pode ser, então, modelado pelo seguinte problema de 
complementaridade:

$$
V_{k}^{\min } \leq V_{k} \leq V_{k}^{\max } \perp \Delta b_{k}^{s h}
$$

Assim como (6.6) foi transformado na associação de dois problemas de complementaridade do tipo (6.4), o problema de complementaridade (8.16) também pode ser modificado da seguinte forma:

$$
\begin{aligned}
& \Delta b_{k}^{s h}=b_{a_{k}}^{s h}-b_{b_{k}}^{s h} \\
& 0 \leq\left(V_{k}-V_{k}^{\text {min }}\right) \perp b_{a_{k}}^{s h} \geq 0 \\
& 0 \leq\left(V_{k}^{\text {max }}-V_{k}\right) \perp b_{b_{k}}^{s h} \geq 0
\end{aligned}
$$

onde $b_{a_{k}}$ e $b_{b_{k}}$ são variáveis auxiliares não negativas que podem, dependendo do valor da magnitude de tensão $V_{k}$, aumentar ou diminuir o ajuste na variável $b_{k}^{s h}$. Portanto, o controle da magnitude de tensão por bancos de capacitores e reatores shunt pode ser modelado algebricamente da seguinte forma:

$$
\begin{aligned}
& \Delta b_{k}^{s h}=b_{a_{k}}^{s h}-b_{b_{k}}^{s h} \\
& \left(V_{k}-V_{k}^{\text {min }}\right) b_{a_{k}}^{s h}=0 \\
& \left(V_{k}^{\text {max }}-V_{k}\right) b_{b_{k}}^{s h}=0 \\
& \left(V_{k}-V_{k}^{\text {min }}\right) \geq 0 \\
& \left(V_{k}^{\text {max }}-V_{k}\right) \geq 0 \\
& b_{a_{k}}^{s h} \geq 0 \\
& b_{b_{k}}^{s h} \geq 0
\end{aligned}
$$

Com a modelagem do controle da magnitude de tensão por transformadores em-fase, bancos de capacitores e reatores shunt apresentada neste capítulo, passa-se agora à formulação do problema de FPOR. 


\section{Capítulo 9}

\section{O Problema de FPOR}

A formulação do problema de FPOR para minimização das perdas ativas na transmissão é apresentada neste capítulo. Inicialmente, são apresentados os modelos clássico e com variáveis de controle discretas. Na sequência, é apresentado o modelo com variáveis de controle discretas e restrições de atuação de dispositivos de controle de tensão proposto neste trabalho.

\subsection{O Problema de FPO}

Um dos objetivos de um FPO é a determinação do ajuste ótimo das variáveis de controle de um SEE em função de um determinado desempenho operacional do sistema. O FPO é um problema de otimização restrita, estático, não convexo, de grande porte e com variáveis contínuas e discretas, cuja formulação pode ser representada da seguinte forma:

$$
\begin{array}{lll}
\min & f(x) & \\
\text { s.a: } & g_{i}(x)=0 & i=1, \cdots, p \\
& h_{i}(x) \leq 0 & i=1, \cdots, q \\
& x_{1_{i}}^{\text {min }} \leq x_{1_{i}} \leq x_{1_{i}}^{\max } & i=1, \cdots, m_{1} \\
& x_{2_{i}} \in \mathcal{D}_{x_{i}} & i=1, \cdots, m_{2}
\end{array}
$$


onde:

- $x_{1} \in \mathbb{R}^{m_{1}}$ é o vetor das variáveis (de controle e dependentes) contínuas do sistema; e $x_{2} \in \mathcal{D}_{x} \subset \mathbb{R}^{m_{2}}$ é o vetor das variáveis de controle discretas, sendo $x=\left(x_{1}, x_{2}\right)$;

- $\mathcal{D}_{x}$ é o conjunto dos valores discretos das variáveis $x_{2}$;

- $f: \mathbb{R}^{m_{1}} \times \mathbb{R}^{m_{2}} \rightarrow \mathbb{R}$ é uma função escalar que representa um desempenho operacional do sistema;

- $g: \mathbb{R}^{m_{1}} \times \mathbb{R}^{m_{2}} \rightarrow \mathbb{R}^{p}$ é o conjunto das restrições de igualdade formado pelas equações do problema de FC, pelas equações do modelo de atuação de dispositivos de controle etc.;

- $h: \mathbb{R}^{m_{1}} \times \mathbb{R}^{m_{2}} \rightarrow \mathbb{R}^{q}$ é o conjunto das restrições de desigualdade formado pelos limites de geração de potência reativa, limites de fluxo de potência nas linhas de transmissão, limites de corrente nos ramos da rede (limites termais) etc.;

- e $x_{1}^{\min }$ e $x_{1}^{\max }$ são, respectivamente, os limites mínimo e máximo das variáveis $x_{1}$.

Devido às dificuldades impostas pela existência das variáveis $x_{2}$ nas formulações de problemas de FPO, a maioria dos modelos propostos na literatura desconsideram sua modelagem discreta, considerando-as contínuas. Nesses casos, a restrição (9.1e) é reformulada da seguinte forma:

$$
x_{2_{i}}^{\min } \leq x_{2_{i}} \leq x_{2_{i}}^{\max }
$$

onde $x_{2}^{\min }$ e $x_{2}^{\max }$ são, respectivamente, os limites mínimo e máximo das variáveis $x_{2}$, sendo $x_{2}^{\min }=\min \left\{\mathcal{D}_{x}\right\}$ e $x_{2}^{\max }=\max \left\{\mathcal{D}_{x}\right\}$. 


\subsection{Formulação do Problema de FPOR}

O parque gerador brasileiro é composto, em sua grande maioria, por usinas hidrelétricas localizadas, geralmente, longe dos grandes centros consumidores, o que requer um amplo sistema de transmissão de energia elétrica (SOUZA, 2005). A transmissão de energia elétrica das fontes geradoras aos centros de carga envolve perdas de potência por efeito Joule nas linhas de transmissão e, dependendo da ordem de grandeza do sistema, a minimização dessas perdas resulta em uma significativa economia na geração do sistema elétrico (BAPTISTA et al., 2006).

Um dos objetivos deste trabalho é o desenvolvimento de um modelo do problema de FPO para a operação de SEEs de grande porte, como o brasileiro, de forma a minimizar as perdas ativas na transmissão. O problema de FPO considerado neste trabalho é o de FPOR, no qual as variáveis de controle associadas à potência ativa são fixas e as variáveis de controle associadas à potência reativa são ajustadas de forma a minimizar as perdas ativas na transmissão.

A seguir são apresentados dois modelos desse problema de FPOR que dão suporte ao desenvolvimento do modelo proposto neste trabalho.

\subsubsection{Modelo Clássico}

Entende-se como modelo clássico do problema de FPOR aquele que otimiza um dado desempenho do sistema, sujeito ao balanço de potência ativa e reativa nas barras da rede, aos limites de geração de potência reativa, e aos limites das variáveis de controle (todas contínuas), como na maioria dos modelos apresentados na literatura. Dessa forma, o modelo clássico do problema de FPOR cujo objetivo consiste na minimização das perdas 
ativas na transmissão é dado por:

$$
\begin{array}{lll}
\min & \sum_{k, m \in \mathcal{L} \cup \mathcal{T}} g_{k m}\left(\frac{1}{t_{k m}^{2}} V_{k}^{2}+V_{m}^{2}-2 \frac{1}{t_{k m}} V_{k} V_{m} \cos \theta_{k m}\right) & \\
\text { s.a: } & P_{k}-\sum_{m \in \mathcal{V}_{k}} P_{k m}(V, \theta, t)=0 & \forall k \in \mathcal{G}^{\prime} \cup \mathcal{C} \\
& Q_{k}+Q_{k}^{s h}\left(V_{k}, b_{k}^{s h}\right)-\sum_{m \in \mathcal{V}_{k}} Q_{k m}(V, \theta, t)=0 & \forall k \in \mathcal{C} \\
Q_{G_{k}}^{\text {min }} \leq Q_{G_{k}}\left(V, \theta, t, b^{s h}\right) \leq Q_{G_{k}}^{\text {max }} & \forall k \in \mathcal{G} \\
V_{k}^{\text {min }} \leq V_{k} \leq V_{k}^{\text {max }} & \forall k \in \mathcal{B} \\
b_{k}^{s h^{\text {min }}} \leq b_{k}^{s h} \leq b_{k}^{s h} \text { max } & \forall k \in \mathcal{B}^{s h} \\
t_{k m}^{\text {min }} \leq t_{k m} \leq t_{k m}^{\text {max }} & \forall k, m \in \mathcal{T}
\end{array}
$$

onde $V$ e $\theta$ são, respectivamente, os vetores das magnitudes e ângulos de fase de tensão nas barras do sistema; $t$ é o vetor dos taps variáveis de transformadores em-fase; e $b^{\text {sh }}$ é o vetor das susceptâncias equivalentes dos bancos de capacitores e reatores shunt. O objetivo de minimizar as perdas ativas na transmissão também pode ser expresso pela minimização da injeção de potência ativa pela barra slack (DOMMEL; TINNEY, 1968). No problema (9.3), $\mathcal{B}^{\text {sh }}$ é o conjunto das barras com controle da magnitude de tensão por bancos de capacitores e reatores shunt, $\mathcal{L}$ é o conjunto das linhas de transmissão, e $\mathcal{T}$ é o conjunto de transformadores com tap variável.

Observe que as equações (9.3b) e (9.3c) equivalem ao conjunto de equações do subsistema 1 do processo de determinação do estado da rede elétrica pela resolução do problema do FC. No entanto, o número de incógnitas do problema de FPO é maior que seu número de restrições de igualdade, o que resulta na possibilidade de infinitas soluções na região factível do problema.

Na solução do problema (9.3) são conhecidos os valores ótimos das magnitudes de tensão das barras de geração, dos ajustes dos taps dos transformadores, e das susceptâncias 
equivalentes dos bancos de capacitores e reatores shunt. Porém, este modelo desconsidera a modelagem discreta dos taps e das susceptâncias equivalentes dos shunts.

\subsubsection{Modelo com Variáveis de Controle Discretas}

Nesta formulação do problema de FPOR, os taps dos transformadores e as susceptâncias equivalentes dos bancos de capacitores e reatores shunt são modelados como variáveis de controle discretas do sistema. Esse modelo de FPOR é dado por:

$$
\begin{aligned}
& \min \sum_{k, m \in \mathcal{L} \cup \mathcal{T}} g_{k m}\left(\frac{1}{t_{k m}^{2}} V_{k}^{2}+V_{m}^{2}-2 \frac{1}{t_{k m}} V_{k} V_{m} \cos \theta_{k m}\right) \\
& \text { s.a: } \quad P_{k}-\sum_{m \in \mathcal{V}_{k}} P_{k m}(V, \theta, t)=0 \quad \forall k \in \mathcal{G}^{\prime} \cup \mathcal{C} \\
& Q_{k}+Q_{k}^{s h}\left(V_{k}, b_{k}^{s h}\right)-\sum_{m \in \mathcal{V}_{k}} Q_{k m}(V, \theta, t)=0 \quad \forall k \in \mathcal{C} \\
& Q_{G_{k}}^{\min } \leq Q_{G_{k}}\left(V, \theta, t, b_{k}^{s h}\right) \leq Q_{G_{k}}^{\max } \quad \forall k \in \mathcal{G} \\
& V_{k}^{\text {min }} \leq V_{k} \leq V_{k}^{\text {max }} \quad \forall k \in \mathcal{B} \\
& b_{k}^{s h} \in \mathcal{D}_{k}^{s h} \quad \forall k \in \mathcal{B}^{s h} \\
& t_{k m} \in \mathcal{D}_{k m}^{t a p} \quad \forall k, m \in \mathcal{T}
\end{aligned}
$$

onde $\mathcal{D}_{k m}^{t a p}$ é o conjunto dos valores discretos dos taps de transformadores, e $\mathcal{D}_{k}^{s h}$ é o conjunto dos valores discretos da susceptância equivalente dos bancos de capacitores e reatores shunt.

Apesar do modelo (9.4) considerar a modelagem discreta dos taps e das susceptâncias equivalentes dos shunts de barra, todas variáveis de controle do sistema ainda são ajustadas para se determinar o estado ótimo da rede. Tal característica pode inviabilizar a aplicação de modelos de FPOR à operação de SEEs de grande porte, pois um número excessivo de ajustes nos controles torna-se praticamente impossível de ser realizado antes de que estado do sistema mude significativamente. 


\subsection{FPOR com Variáveis de Controle Discretas e Restrições de Atuação de Dispositivos de Controle de Tensão}

A partir do estudo realizado acerca dos modelos e abordagens para resolução de problemas de FPO, o modelo para o problema de FPOR com variáveis de controle discretas e restrições de atuação de dispositivos de controle de tensão proposto neste trabalho é:

$$
\begin{array}{lll}
\min & \sum_{k, m \in \mathcal{L} \cup \mathcal{T}} g_{k m}\left(\frac{1}{t_{k m}^{2}} V_{k}^{2}+V_{m}^{2}-2 \frac{1}{t_{k m}} V_{k} V_{m} \cos \theta_{k m}\right) & \\
\text { s.a: } & P_{k}-\sum_{m \in \mathcal{V}_{k}} P_{k m}(V, \theta, t)=0 & \forall k \in \mathcal{G}^{\prime} \cup \mathcal{C} \\
Q_{k}+Q_{k}^{s h}\left(V_{k}, b_{k}^{s h}\right)-\sum_{m \in \mathcal{V}_{k}} Q_{k m}(V, \theta, t)=0 & \forall k \in \mathcal{C} \\
Q_{G_{k}}^{\text {min }} \leq Q_{G_{k}}(V, \theta, t) \leq Q_{G_{k}}^{\text {max }} & \forall k \in \mathcal{G} \\
V_{k}^{\text {min }} \leq V_{k} \leq V_{k}^{\text {max }} & \forall k \in \mathcal{B} \\
b_{k}^{s h} \in \mathcal{D}^{s h} & \forall k \in \mathcal{B}^{s h} \\
V_{k}^{\text {min }} \leq V_{k} \leq V_{k}^{\text {max }} \perp \Delta b_{k}^{s h} & \forall k \in \mathcal{B}^{s h} \\
t_{k m} \in \mathcal{D}_{\text {tap }} & \forall k, m \in \mathcal{T} \\
V_{k}^{\text {min }} \leq V_{k} \leq V_{k}^{\text {max }} \perp-\Delta t_{k m} & \forall k, m \in \mathcal{T} \mid k \in \mathcal{T}_{z} \\
V_{k}^{\text {min }} \leq V_{k} \leq V_{k}^{\text {max }} \perp \Delta t_{k m} & \forall k, m \in \mathcal{T} \mid k \in \mathcal{T}_{\text {tap }}
\end{array}
$$

A presença de variáveis de controle discretas, restrições não lineares e restrições de complementaridade deste modelo dificultam sua solução. Problemas de FPOR como o representado em (9.5) possuem características de problemas de PNL e de PIM, além de possuírem características próprias que não aparecem nesses dois tipos de problema. 
A seguir são apresentados os testes realizados para verificar a eficácia do modelo (9.5) e a robustez da abordagem para resolução de problemas de FPOR pelo método da FLBMP. 


\section{Capítulo 10}

\section{Resultados Numéricos}

Neste capítulo são apresentados os resultados numéricos obtidos através da aplicação do método da FLBMP para resolução dos problemas de FPOR dados pelos modelos clássico (9.3), com variáveis de controle discretas (9.4), e com variáveis de controle discretas e restrições de atuação de dispositivos de controle de tensão (9.5) para os sistemas IEEE de 14, 30, 57 e 118 barras (POWER..., 1999) e o sistema equivalente CESP 440 kV de 53 barras.

Esses resultados foram obtidos a partir da implementação da FLBMP associada aos modelos (9.3), (9.4) e (9.5) na linguagem para modelagem de problemas matemáticos AMPL (FOURER; GAY; KERNIGHAN, 2002). As condições necessárias de otimalidade do problema de barreira modificada-penalidade resultante foram resolvidas pelo solver IPOPT (WÄCHTER; BIEGLER, 2006; INTERIOR..., 2011).

\subsection{Sistema IEEE de 14 Barras}

O sistema IEEE de 14 barras possui as seguintes características:

- 1 barra slack;

- 4 barras com controle de geração de reativos;

- 9 barras de carga; 
Tabela 10.1 - Limites mínimo e máximo da magnitude de tensão das barras controladas do sistema IEEE de 14 barras.

\begin{tabular}{cccc}
\hline $\begin{array}{c}\text { Barra } \\
\text { Controlada }\end{array}$ & $\begin{array}{c}\text { Variável } \\
\text { de Controle }\end{array}$ & $\begin{array}{c}V_{k}^{\text {min }} \\
\text { (p.u.) }\end{array}$ & $\begin{array}{l}V_{k}^{\max } \\
\text { (p.u.) }\end{array}$ \\
\hline 9 & $b_{9}^{\text {sh }}$ & 1,045 & 1,065 \\
6 & $t_{5-6}$ & 1,060 & 1,080 \\
7 & $t_{4-7}$ & 1,050 & 1,070 \\
9 & $t_{4-9}$ & 1,045 & 1,065 \\
\hline
\end{tabular}

- 1 banco de capacitores;

- 17 linhas de transmissão;

- 3 transformadores com tap variável.

Para esse sistema, considerou-se que os limites mínimo e máximo da magnitude de tensão fossem, respectivamente, 0,950 e 1,100 p.u. Os limites mínimo e máximo da magnitude de tensão das barras controladas pelos transformadores em-fase e pelo banco de capacitores foram considerados iguais a $\pm 0,01$ p.u. do valor da magnitude especificada no banco de dados e são dados na Tabela 10.1.

Na modelagem das variáveis de controle discretas, considerou-se que o tamanho do passo entre dois valores consecutivos dos taps dos transformadores fosse 0,01 p.u. e que seus limites mínimo e máximo fossem 0,880 e 1,120 p.u. Por fim, considerou-se que o banco de capacitores da barra 9 fosse um regulador de tensão formado pela associação em paralelo de três capacitores: um de 5,0 MVAr, um de 15,0 MVAr e um de 19,0 MVAr na tensão nominal. Dessa forma, considerou-se que os valores discretos que a susceptância equivalente desse shunt pode assumir na solução do problema são dados por todas as combinações simples possíveis entre as susceptâncias desses capacitores, ou seja:

$$
b_{9}^{s h} \in\{0 ; 0,05 ; 0,15 ; 0,19 ; 0,20 ; 0,24 ; 0,34 ; 0,39\}
$$


Tabela 10.2 - Valores ótimos das variáveis de controle do sistema IEEE de 14 barras determinados pelo modelo (9.3).

\begin{tabular}{ccc}
\hline $\begin{array}{c}\text { Barra } \\
(\mathrm{k})\end{array}$ & $\begin{array}{c}V_{k} \\
\text { (p.u. })\end{array}$ & $\begin{array}{c}b_{k}^{s h} \\
\text { (p.u. })\end{array}$ \\
\hline 1 & 1,100 & - \\
2 & 1,086 & - \\
3 & 1,057 & - \\
6 & 1,072 & - \\
8 & 1,045 & - \\
9 & - & 0,390 \\
\hline Da Barra & Para Barra & $t_{k m}$ \\
$(\mathrm{k})$ & $(\mathrm{m})$ & $(\mathrm{p} . u)$. \\
\hline 4 & 7 & 1,0349 \\
4 & 9 & 1,0094 \\
5 & 6 & 1,0052 \\
\hline \multicolumn{3}{r}{ Perdas $=12,3095 \mathrm{MW}$} \\
\hline \multicolumn{3}{c}{}
\end{tabular}

\subsubsection{Modelo Clássico}

Resolvendo-se o problema de FPOR para o sistema IEEE de 14 barras pelo modelo (9.3), determinam-se os ajustes ótimos das magnitudes de tensão das barras de geração, dos taps variáveis dos transformadores e da susceptância equivalente do banco de capacitores. Neste modelo, os taps dos transformadores e a susceptância equivalente do banco de capacitores são considerados variáveis contínuas, cujos limites mínimo e máximo são, respectivamente, 0,880 e 1,120 p.u., e 0 e 0,39 p.u.

Os valores ótimos dessas variáveis de controle são apresentados na Tabela 10.2. Pela aplicação deste modelo, as perdas ativas na transmissão foram reduzidas em $8,17 \%$, mantendo-se um perfil de tensão médio em torno de 1,0629 p.u.

De acordo com o modelo (9.3), os taps dos transformadores e o banco de capacitores são ajustados em função da minimização das perdas ativas no sistema de transmissão. 


\subsubsection{Modelo com Variáveis de Controle Discretas}

No modelo (9.4), os taps dos transformadores e a susceptância equivalente do shunt da barra 9 são modelados como variáveis de controle discretas.

De acordo com o algoritmo da FLBMP-discreto aplicado à resolução de (9.4) para o sistema IEEE de 14 barras:

- o valor inicial do parâmetro $\gamma$ das funções $\psi$ associadas aos taps dos transformadores foi $10^{-8}$;

- o valor inicial do parâmetro $\gamma$ da função $\psi$ associada ao shunt da barra 9 foi $10^{-7}$;

- a constante $\beta$ foi considerada igual a 1 ;

- o fator $\tau$ de ajuste de $\gamma$ foi fixado em 10;

- e a tolerância $\xi$ para convergência do algoritmo da FLBMP-discreto foi de $10^{-4}$.

Os valores ótimos das variáveis de controle determinados pela resolução do problema (9.4) para o sistema IEEE de 14 barras são apresentados na Tabela 10.3. Pela aplicação deste modelo, as perdas ativas neste sistema foram reduzidas em 8,15\%, mantendo-se um perfil de tensão médio em torno de 1,0630 p.u.

As Figuras 10.1, 10.2 e 10.3 mostram, respectivamente, os valores da susceptância equivalente do shunt da barra 9, dos taps dos transformadores e da função perdas ao longo do processo iterativo de resolução do problema (9.4) pelo algoritmo da FLBMP-discreto. O pequeno aumento que se observa na função perdas (de 12,3095 MW para 12,3106 MW) se deve ao ajuste dos taps dos transformadores para seus valores discretos. Como pode ser visto na Figura 10.1, a susceptância equivalente do shunt da barra 9 permanece no seu limite superior durante todo o processo iterativo. 
Tabela 10.3 - Valores ótimos das variáveis de controle do sistema IEEE de 14 barras determinados pelo modelo (9.4).

\begin{tabular}{ccc}
\hline $\begin{array}{c}\text { Barra } \\
(\mathrm{k})\end{array}$ & $\begin{array}{c}V_{k} \\
(\mathrm{p} . u .)\end{array}$ & $\begin{array}{c}b_{k}^{s h} \\
\text { (p.u. })\end{array}$ \\
\hline 1 & 1,100 & - \\
2 & 1,086 & - \\
3 & 1,057 & - \\
6 & 1,069 & - \\
8 & 1,050 & - \\
9 & - & 0,390 \\
\hline Da Barra & Para Barra & $t_{k m}$ \\
$(\mathrm{k})$ & $(\mathrm{m})$ & $(\mathrm{p} . u)$. \\
\hline 4 & 7 & 1,0300 \\
4 & 9 & 1,0200 \\
5 & 6 & 1,0100 \\
\hline \multicolumn{3}{c}{ Número de iterações $=8$} \\
\hline \multicolumn{3}{r}{ Perdas $=12,3106 \mathrm{MW}$} \\
\hline \multicolumn{3}{c}{}
\end{tabular}

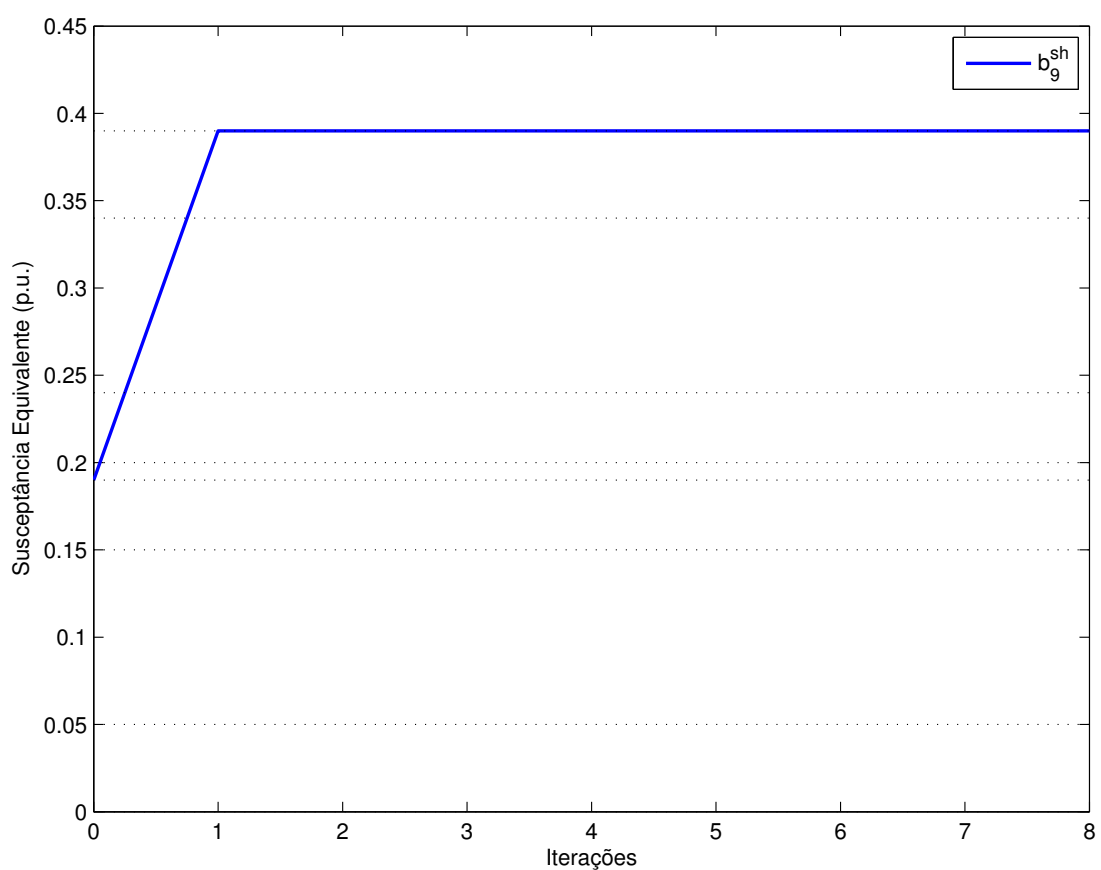

Figura 10.1 - Valor da susceptância equivalente do banco de capacitores do sistema IEEE de 14 barras ao longo do processo de resolução do FPOR (9.4). 


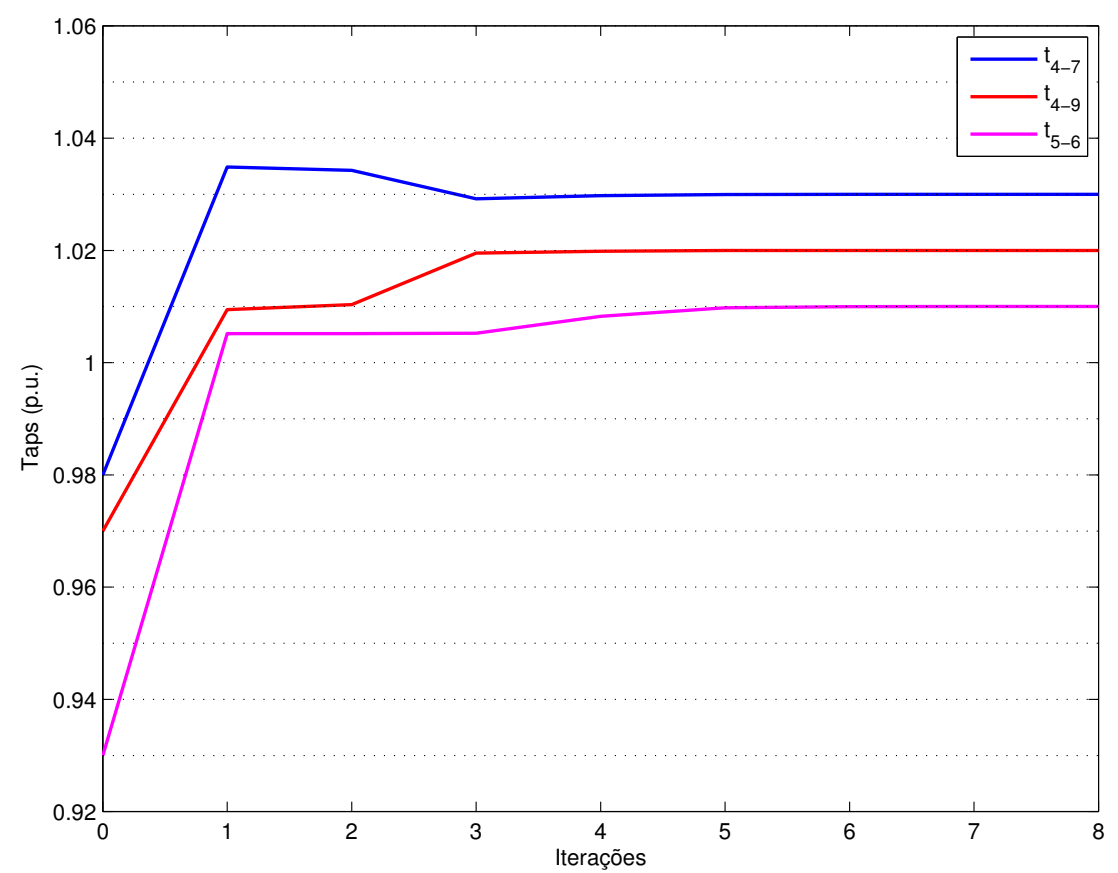

Figura 10.2 - Valores dos taps dos transformadores do sistema IEEE 14 barras ao longo do processo de resolução do FPOR (9.4).

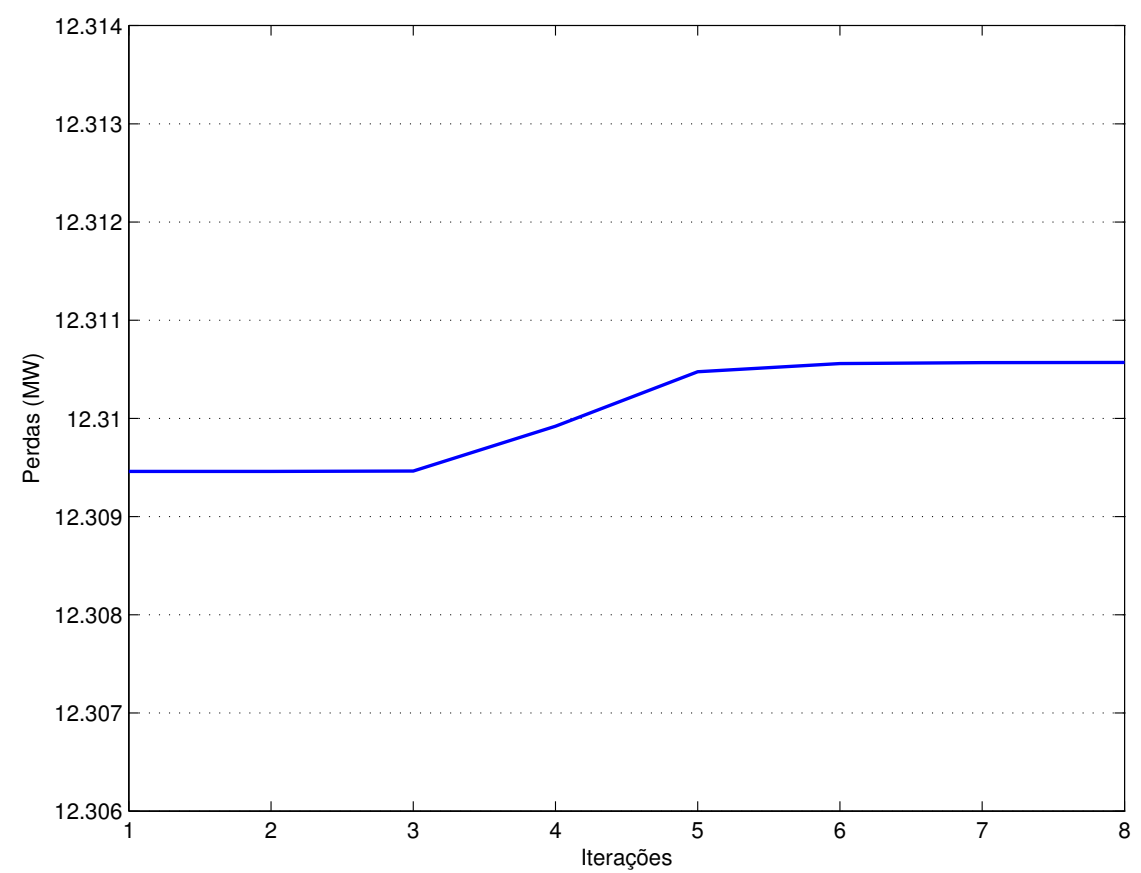

Figura 10.3 - Perdas ativas no sistema IEEE de 14 barras ao longo do processo de resolução do FPOR (9.4). 


\subsubsection{Modelo com Variáveis de Controle Discretas e Restrições de Atuação de Dispositivos de Controle de Tensão}

Pelo modelo (9.5), os taps dos transformadores e as susceptâncias equivalentes dos bancos de capacitores e reatores shunt são ajustados automaticamente somente quando um dos limites da magnitude de tensão da barra controlada por esses dispositivos for atingido. Os limites da magnitude de tensão das barras controladas pelos transformadores e pelo banco de capacitores do sistema IEEE de 14 barras são dados na Tabela 10.1. Observe que a barra 9 possui dois dispositivos de controle: um transformador e um banco de capacitores.

De acordo com o algoritmo da FLBMP-discreto aplicado à resolução de (9.5) para o sistema IEEE de 14 barras:

- o valor inicial do parâmetro $\gamma$ das funções $\psi$ associadas aos taps dos transformadores foi $10^{-5}$;

- o valor inicial do parâmetro $\gamma$ da função $\psi$ associada ao shunt da barra 9 foi $10^{-5}$;

- a constante $\beta$ foi considerada igual a 1 ;

- o fator $\tau$ de ajuste de $\gamma$ foi fixado em 10;

- e a tolerância $\xi$ para convergência do algoritmo da FLBMP-discreto foi de $10^{-4}$.

Como as variáveis de controle discretas do modelo (9.5) podem permanecer inalteradas durante o processo de resolução do problema e as mesmas são determinadas em função de um valor inicial associado ao caso base, os valores especificados para essas variáveis de controle no caso base devem, portanto, pertencer ao conjunto dos valores discretos que essa variável pode assumir. Caso tal condição não seja obedecida, o processo iterativo de resolução do problema (9.5) pelo algoritmo da FLBMP-discreto não convergirá. Os valores iniciais considerados para as variáveis de controle discretas do sistema IEEE de 14 barras no caso base foram: $t_{4-7_{o}}=0,980$ p.u.; $t_{4-9_{o}}=0,970$ p.u.; $t_{5-6_{o}}=0,930$ p.u.; e $b_{9_{o}}^{s h}=0,190$ p.u. 
Tabela 10.4 - Valores ótimos das variáveis de controle do sistema IEEE de 14 barras determinados pelo modelo (9.5).

\begin{tabular}{ccccc}
\hline $\begin{array}{c}\text { Barra } \\
(\mathrm{k})\end{array}$ & $\begin{array}{c}V_{k} \\
(\mathrm{p} . u .)\end{array}$ & $\begin{array}{c}b_{k}^{s h} \\
(\mathrm{p} . u .)\end{array}$ & $\begin{array}{c}b_{a_{k}} \\
(\mathrm{p} . u .)\end{array}$ & $\begin{array}{c}b_{b_{k}} \\
(\mathrm{p} . u .)\end{array}$ \\
\hline 1 & 1,100 & - & - & - \\
2 & 1,089 & - & - & - \\
3 & 1,062 & - & - & - \\
6 & 1,080 & - & - & - \\
8 & 1,100 & - & - & - \\
9 & - & 0,190 & 0,000 & 0,000 \\
\hline Da Barra & Para Barra & $t_{k m}$ & $t_{a_{k m}}$ & $t_{b_{k m}}$ \\
$(\mathrm{k})$ & $(\mathrm{m})$ & $(\mathrm{p} . \mathrm{u})$. & $(\mathrm{p} . u)$. & $(\mathrm{p} . \mathrm{u})$. \\
\hline 4 & 7 & 1,0200 & 0,0000 & 0,0400 \\
4 & 9 & 1,0100 & 0,0000 & 0,0400 \\
5 & 6 & 0,9900 & 0,0000 & 0,0600 \\
\hline \multicolumn{5}{c}{ Número de iterações $=6$} \\
\hline \multicolumn{5}{c}{ Perdas = 12,3328 MW }
\end{tabular}

Os valores ótimos das variáveis de controle determinados pela resolução do problema (9.5) para o sistema IEEE de 14 barras são apresentados na Tabela $10.4^{1}$. Pela aplicação deste modelo, as perdas ativas neste sistema de transmissão foram reduzidas em 7,99\%, mantendo-se um perfil de tensão médio em torno de 1,0714 p.u. Observe que somente a variável $t_{4-9}$ é ajustada de forma a manter a tensão da barra 9 dentro dos limites especificados na Tabela 10.1.

As Figuras 10.4, 10.5 e 10.6 mostram, respectivamente, os valores da susceptância equivalente do shunt da barra 9, dos taps dos transformadores e da função perdas ao longo do processo iterativo de resolução do problema (9.5) pelo algoritmo da FLBMP-discreto. O aumento que se observa na função perdas (de 12,3222 MW para 12,3328 MW) se deve ao ajuste dos taps dos transformadores para seus valores discretos ótimos em função do controle da magnitude de tensão de uma das suas barras adjacentes. A Figura 10.7 mostra os perfis das magnitudes de tensão dos pontos ótimos determinados por cada FPOR.

\footnotetext{
${ }^{1}$ Os relatórios dos fluxos ótimos deste e dos demais sistemas IEEE são apresentados no Apêndice B.
} 


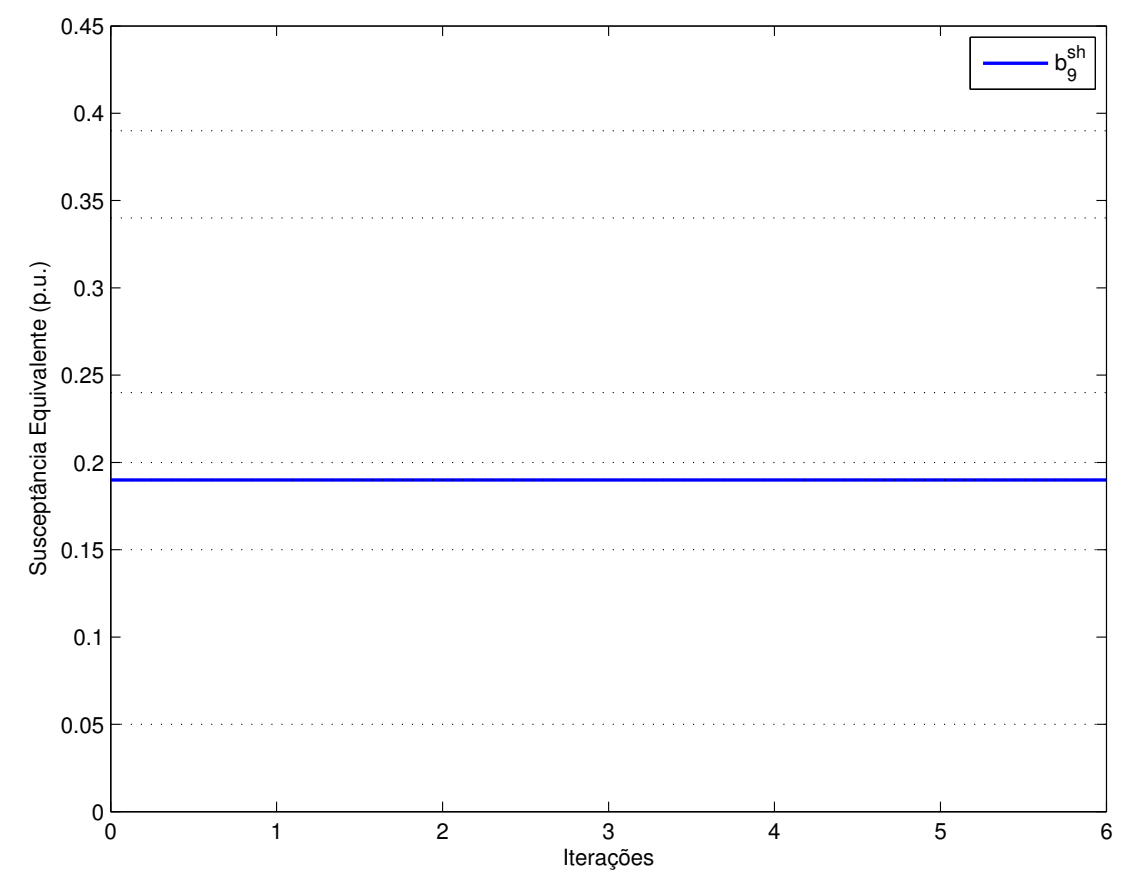

Figura 10.4 - Valor da susceptância equivalente do banco de capacitores do sistema IEEE de 14 barras ao longo do processo de resolução do FPOR (9.5).

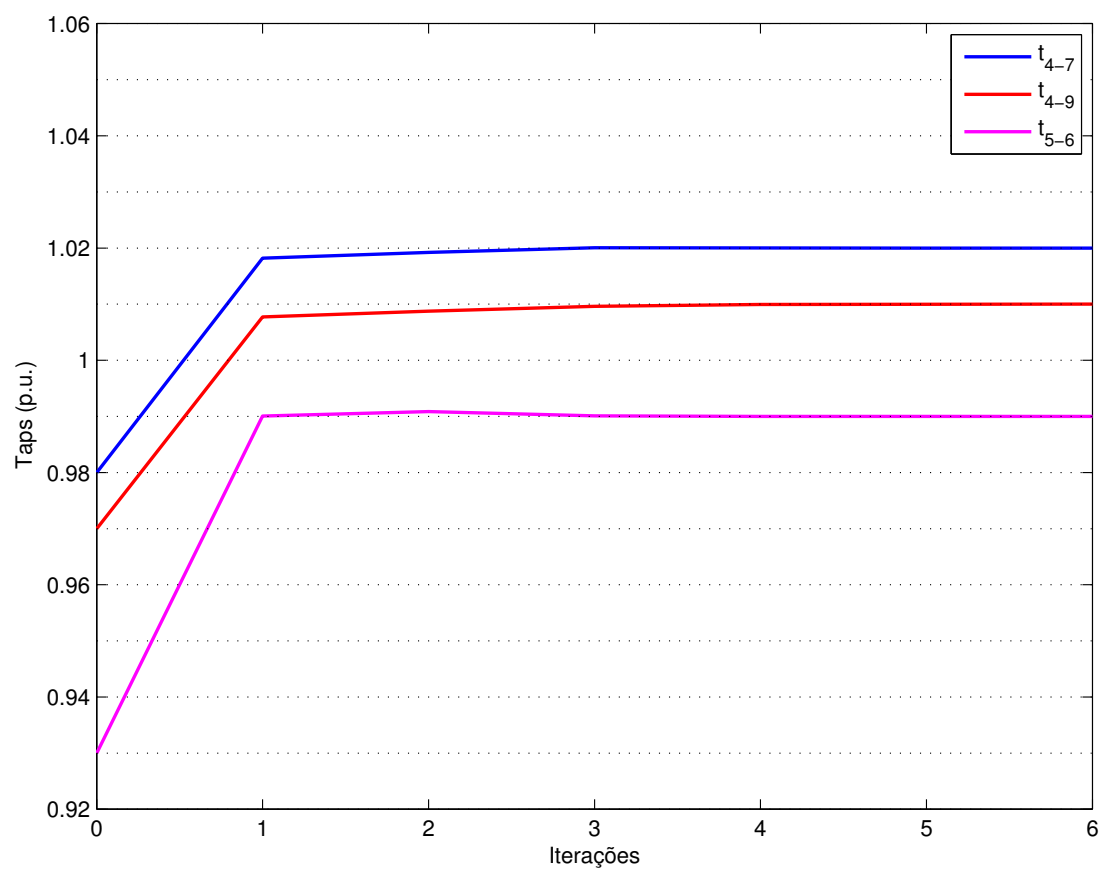

Figura 10.5 - Valores dos taps dos transformadores do sistema IEEE de 14 barras ao longo do processo de resolução do modelo (9.5). 


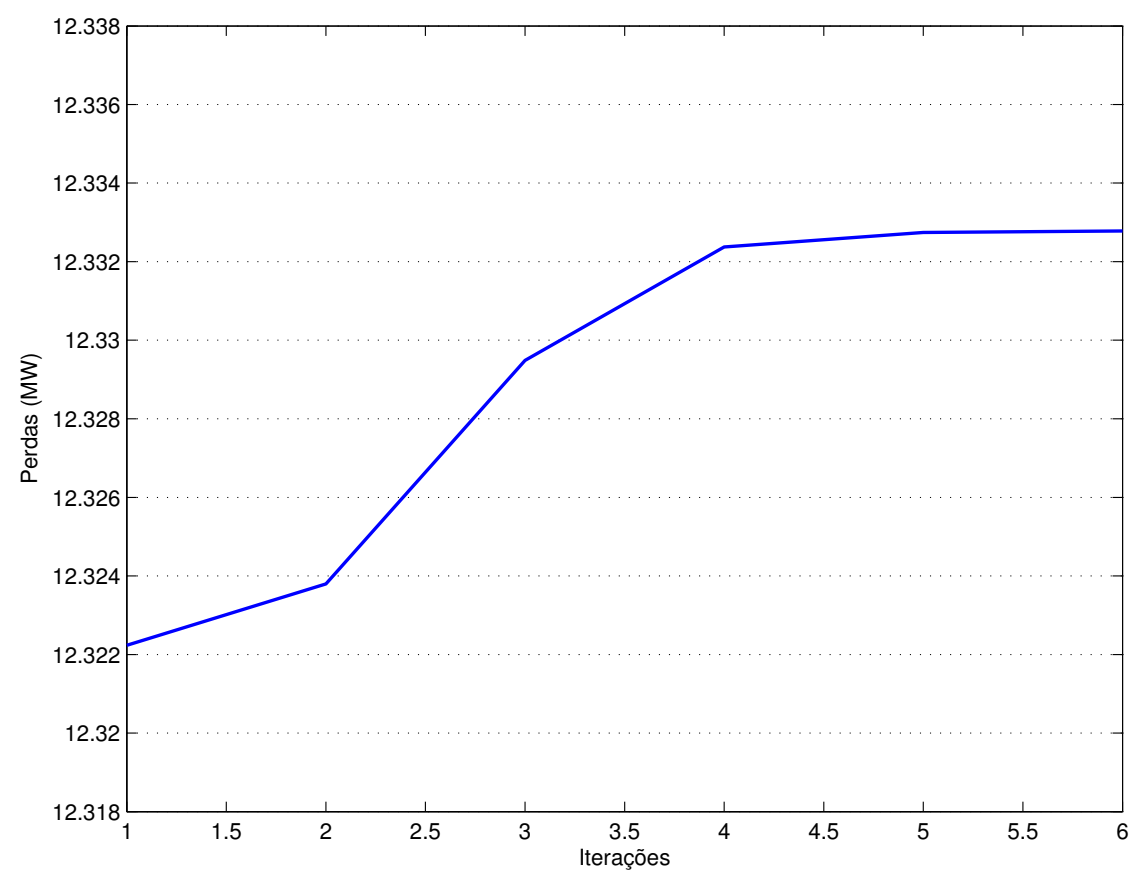

Figura 10.6 - Perdas ativas no sistema IEEE de 14 barras ao longo do processo de resolução do modelo (9.5).

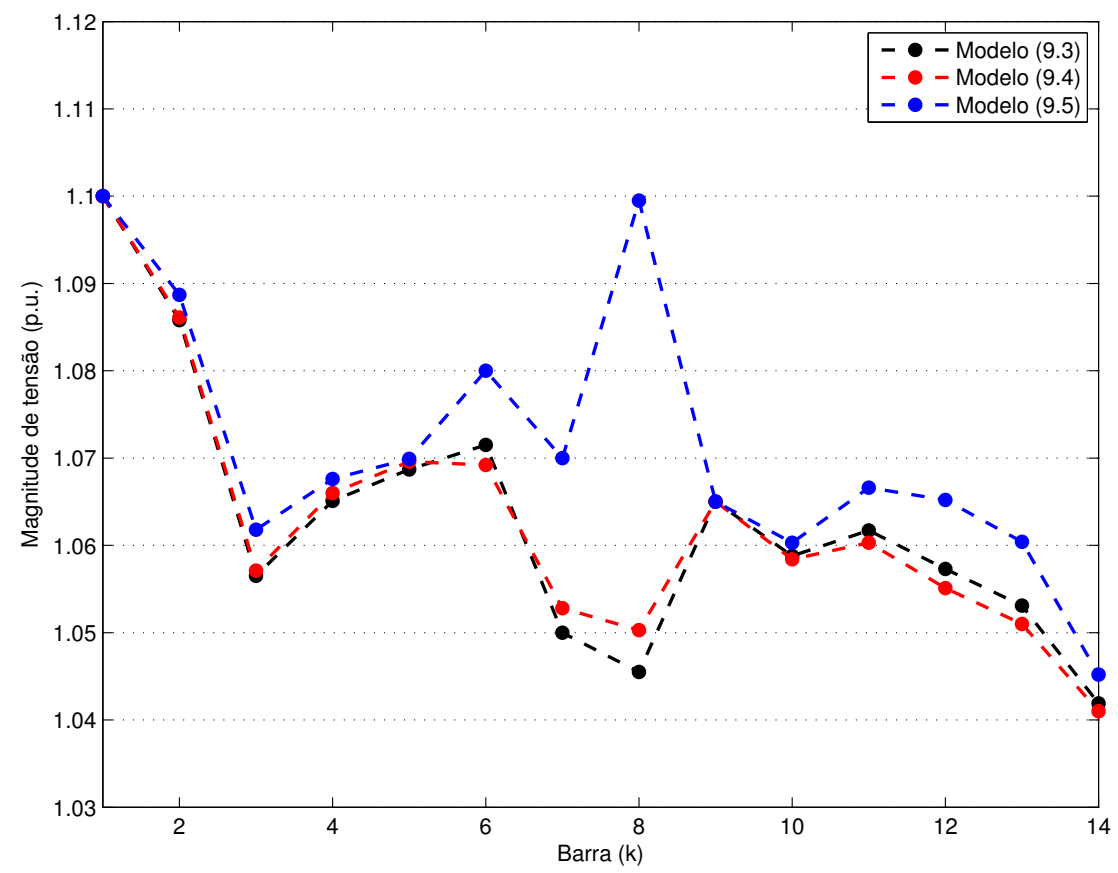

Figura 10.7 - Perfis das magnitudes de tensão determinados pelos modelos (9.3), (9.4) e (9.5) para o sistema IEEE de 14 barras. 
Tabela 10.5 - Variáveis de controle em função dos limites mínimo e máximo da magnitude de tensão na barra 9 .

\begin{tabular}{|c|c|c|c|c|c|c|c|c|c|}
\hline $\begin{array}{l}V_{9}^{\min } \\
\text { (p.u.) }\end{array}$ & $\begin{array}{c}V_{9} \\
\text { (p.u.) }\end{array}$ & $\begin{array}{l}V_{9}^{\max } \\
\text { (p.u.) }\end{array}$ & $\begin{array}{c}t_{4-9} \\
\text { (p.u.) }\end{array}$ & $\begin{array}{l}t_{a_{4-9}} \\
\text { (p.u.) }\end{array}$ & $\begin{array}{l}t_{b_{4-9}} \\
\text { (p.u.) }\end{array}$ & $\begin{array}{c}b_{9}^{s h} \\
\text { (p.u.) }\end{array}$ & $\begin{array}{c}b_{a_{9}} \\
\text { (p.u.) }\end{array}$ & $\begin{array}{c}b_{b_{9}} \\
\text { (p.u.) }\end{array}$ & $\begin{array}{l}\text { Perdas } \\
(\mathrm{MW})\end{array}$ \\
\hline 1,090 & 1,090 & 1,100 & 0,950 & 0,020 & 0,000 & 0,390 & 0,200 & 0,000 & 12,3442 \\
\hline 1,087 & 1,087 & 1,090 & 0,970 & 0,000 & 0,000 & 0,390 & 0,200 & 0,000 & 12,3439 \\
\hline 1,070 & 1,070 & 1,080 & 0,970 & 0,000 & 0,000 & 0,190 & 0,000 & 0,000 & 12,3240 \\
\hline 1,050 & 1,060 & 1,060 & 1,040 & 0,000 & 0,070 & 0,190 & 0,000 & 0,000 & 12,3613 \\
\hline 1,025 & 1,035 & 1,035 & 1,120 & 0,000 & 0,150 & 0,150 & 0,000 & 0,040 & 12,6489 \\
\hline 1,010 & 1,020 & 1,020 & 1,120 & 0,000 & 0,150 & 0,050 & 0,000 & 0,140 & 12,8113 \\
\hline 1,000 & 1,010 & 1,010 & 1,120 & 0,000 & 0,150 & 0,000 & 0,000 & 0,190 & 14,2299 \\
\hline
\end{tabular}

De acordo com os limites das magnitudes de tensão controladas dados na Tabela 10.1, os ajustes na variável $t_{4-9}$ são "suficientes" para se controlar a magnitude de tensão da barra 9 e a variável $b_{9}^{\text {sh }}$ permanece inalterada. Na Tabela 10.5 são apresentados os valores dessas variáveis de controle determinados em função de outros limites de magnitude de tensão da barra 9 .

\subsection{Sistema IEEE de 30 Barras}

O sistema IEEE de 30 barras possui as seguintes características:

- 1 barra slack;

- 5 barras com controle de geração de reativos;

- 24 barras de carga;

- 2 bancos de capacitores;

- 37 linhas de transmissão;

- 4 transformadores com tap variável. 
Tabela 10.6 - Limites mínimo e máximo da magnitude de tensão das barras controladas do sistema IEEE de 30 de barras.

\begin{tabular}{cccc}
\hline $\begin{array}{c}\text { Barra } \\
\text { Controlada }\end{array}$ & $\begin{array}{c}\text { Variável } \\
\text { de Controle }\end{array}$ & $\begin{array}{c}V_{k}^{\min } \\
\text { (p.u.) }\end{array}$ & $\begin{array}{l}V_{k}^{\max } \\
\text { (p.u.) }\end{array}$ \\
\hline 10 & $b_{10}^{s h}$ & 1,045 & 1,065 \\
24 & $b_{24}^{s h}$ & 1,011 & 1,031 \\
9 & $t_{6-9}$ & 1,050 & 1,070 \\
10 & $t_{6-10}$ & 1,045 & 1,065 \\
12 & $t_{4-12}$ & 1,060 & 1,080 \\
27 & $t_{28-27}$ & 1,015 & 1,035 \\
\hline
\end{tabular}

Para esse sistema, considerou-se que os limites mínimo e máximo da magnitude de tensão fossem, respectivamente, 0,950 e 1,100 p.u. Os limites mínimo e máximo da magnitude de tensão das barras controladas pelos transformadores em-fase e pelos bancos de capacitores foram considerados iguais a $\pm 0,01$ p.u. do valor da magnitude especificada no banco de dados e são dados na Tabela 10.6.

Na modelagem das variáveis de controle discretas, considerou-se que o tamanho do passo entre dois valores consecutivos dos taps dos transformadores fosse 0,01 p.u. e que seus limites mínimo e máximo fossem 0, 880 e 1,120 p.u. Por fim, considerou-se que o banco de capacitores da barra 10 é um regulador de tensão formado pela associação em paralelo de três capacitores: um de 5,0 MVAr, um de 15,0 MVAr e um de 19,0 MVAr na tensão nominal. O banco de capacitores da barra 24 é formado pela associação em paralelo de dois capacitores: um de 4,0MVAr e um de 5,0 MVAr na tensão nominal. Assim como para o sistema IEEE de 14 barras, considerou-se que os valores discretos que as susceptâncias equivalentes desses shunts podem assumir na solução do problema são dados por todas as combinações simples possíveis entre as susceptâncias desses capacitores, ou seja:

$$
\begin{aligned}
& b_{10}^{s h} \in\{0 ; 0,05 ; 0,15 ; 0,19 ; 0,20 ; 0,24 ; 0,34 ; 0,39\} \\
& b_{24}^{s h} \in\{0 ; 0,04 ; 0,05 ; 0,09\}
\end{aligned}
$$


Tabela 10.7 - Valores ótimos das variáveis de controle do sistema IEEE de 30 barras determinados pelo modelo (9.3).

\begin{tabular}{ccc}
\hline $\begin{array}{c}\text { Barra } \\
(\mathrm{k})\end{array}$ & $\begin{array}{c}V_{k} \\
\text { (p.u. })\end{array}$ & $\begin{array}{c}b_{k}^{s h} \\
(\mathrm{p} . \mathrm{u} .)\end{array}$ \\
\hline 1 & 1,100 & - \\
2 & 1,085 & - \\
5 & 1,053 & - \\
8 & 1,059 & - \\
10 & - & 0,366 \\
11 & 1,083 & - \\
13 & 1,091 & - \\
24 & - & 0,074 \\
\hline Da Barra & Para Barra & $t_{k m}$ \\
$(\mathrm{k})$ & $(\mathrm{m})$ & $(\mathrm{p} . \mathrm{u})$. \\
\hline 6 & 9 & 1,0384 \\
6 & 10 & 1,1200 \\
4 & 12 & 1,0083 \\
28 & 27 & 1,0054 \\
\hline Perdas $=16,0771 \mathrm{MW}$
\end{tabular}

\subsubsection{Modelo Clássico}

Pela aplicação do modelo (9.3), as perdas ativas na transmissão foram reduzidas em 8,40\%, mantendo-se um perfil de tensão médio em torno de 1,0479 p.u. Os valores ótimos das magnitudes de tensão das barras de geração, dos taps dos transformadores e das susceptâncias equivalentes dos bancos de capacitores são apresentados na Tabela 10.7.

\subsubsection{Modelo com Variáveis de Controle Discretas}

De acordo com o algoritmo da FLBMP-discreto aplicado à resolução de (9.4) para o sistema IEEE de 30 barras:

- os valores iniciais dos parâmetros $\gamma$ das funções $\psi$ associadas aos taps dos transformadores e aos shunts variáveis foram, respectivamente, $10^{-8}$ e $10^{-7}$; 
Tabela 10.8 - Valores ótimos das variáveis de controle do sistema IEEE de 30 barras determinados pelo modelo (9.4).

\begin{tabular}{|c|c|c|}
\hline $\begin{array}{c}\text { Barra } \\
(\mathrm{k})\end{array}$ & $\begin{array}{c}V_{k} \\
\text { (p.u.) } \\
\end{array}$ & $\begin{array}{c}b_{k}^{s h} \\
\text { (p.u.) }\end{array}$ \\
\hline 1 & 1,100 & - \\
\hline 2 & 1,088 & - \\
\hline 5 & 1,058 & - \\
\hline 8 & 1,060 & - \\
\hline 10 & - & 0,340 \\
\hline 11 & 1,083 & - \\
\hline 13 & 1,091 & - \\
\hline 24 & - & 0,090 \\
\hline $\begin{array}{c}\text { Da Barra } \\
(\mathrm{k})\end{array}$ & $\begin{array}{c}\text { Para Barra } \\
(\mathrm{m})\end{array}$ & $\begin{array}{c}t_{k m} \\
\text { (p.u.) }\end{array}$ \\
\hline 6 & 9 & 1,0400 \\
\hline 6 & 10 & 1,1100 \\
\hline 4 & 12 & 1,0100 \\
\hline 28 & 27 & 1,0200 \\
\hline \multicolumn{3}{|c|}{ Número de iterações $=10$} \\
\hline Perda & $s=16,1044 \mathrm{~N}$ & \\
\hline
\end{tabular}

- a constante $\beta$ foi considerada igual a 1 ;

- o fator $\tau$ de ajuste de $\gamma$ foi fixado em 10;

- e a tolerância $\xi$ para convergência do algoritmo da FLBMP-discreto foi de $10^{-4}$.

Os valores ótimos das variáveis de controle determinados pela resolução do problema (9.4) para o sistema IEEE de 30 barras são apresentados na Tabela 10.8. Pela aplicação deste modelo, as perdas ativas neste sistema foram reduzidas em 8,24\%, mantendo-se um perfil de tensão médio em torno de 1,0475 p.u.

As Figuras 10.8, 10.9 e 10.10 mostram, respectivamente, os valores das susceptâncias equivalentes dos shunts das barras 10 e 24, dos taps dos transformadores e da função perdas ao longo do processo iterativo de resolução do problema (9.4) pelo algoritmo da FLBMP-discreto. 


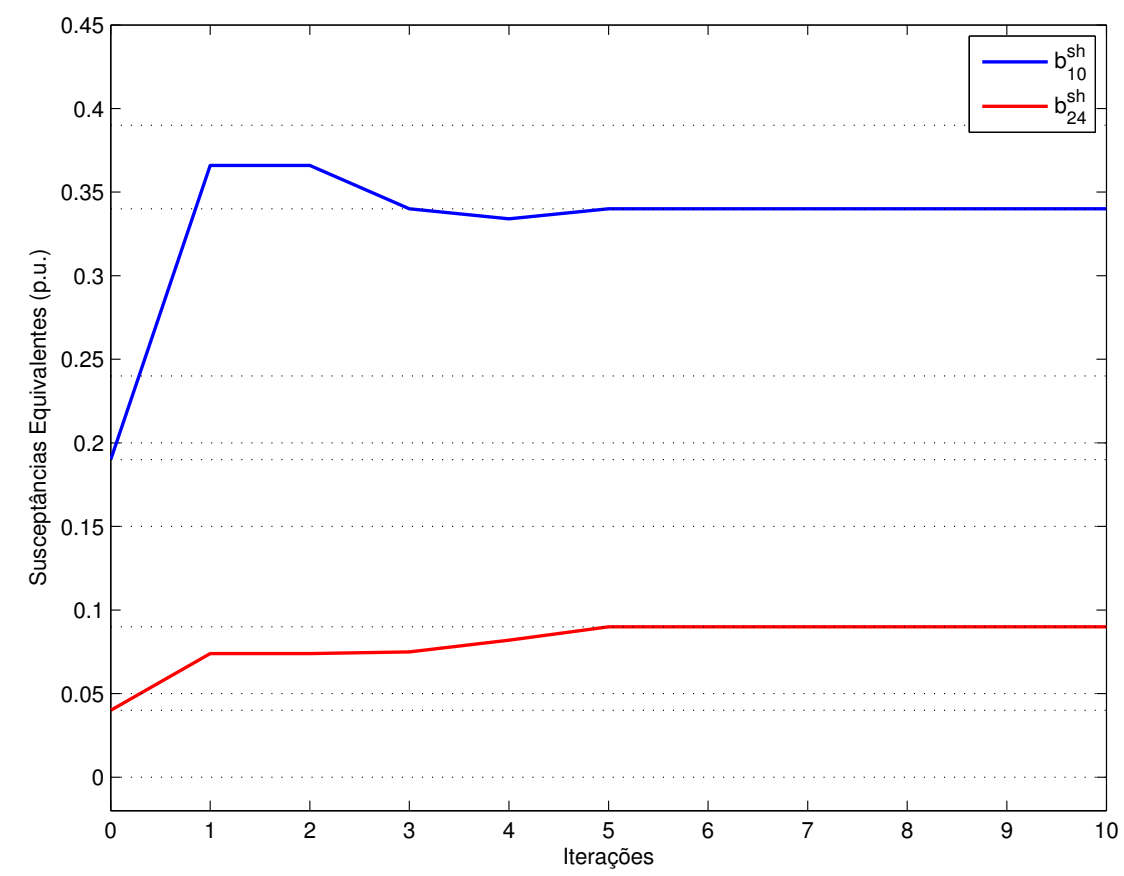

Figura 10.8 - Valores das susceptâncias equivalentes dos bancos de capacitores do sistema IEEE de 30 barras ao longo do processo de resolução do FPOR (9.4).

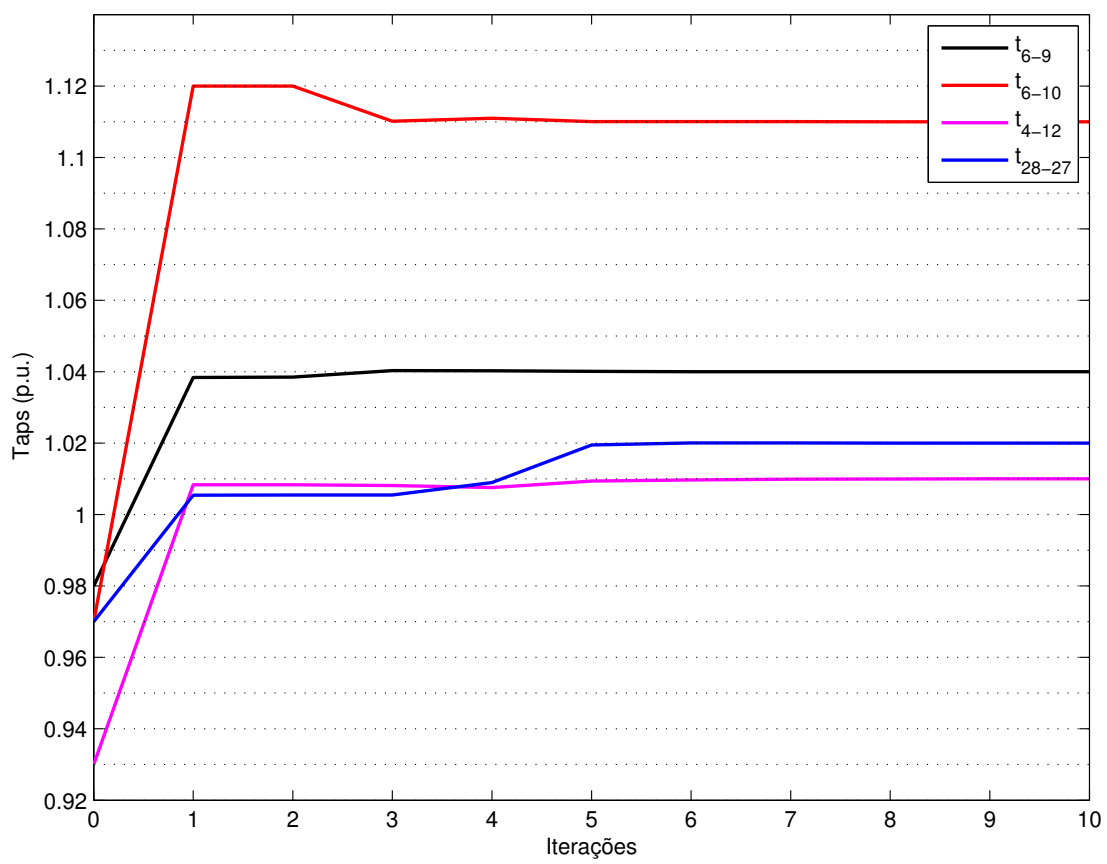

Figura 10.9 - Valores dos taps dos transformadores do sistema IEEE de 30 barras ao longo do processo de resolução do FPOR (9.4). 


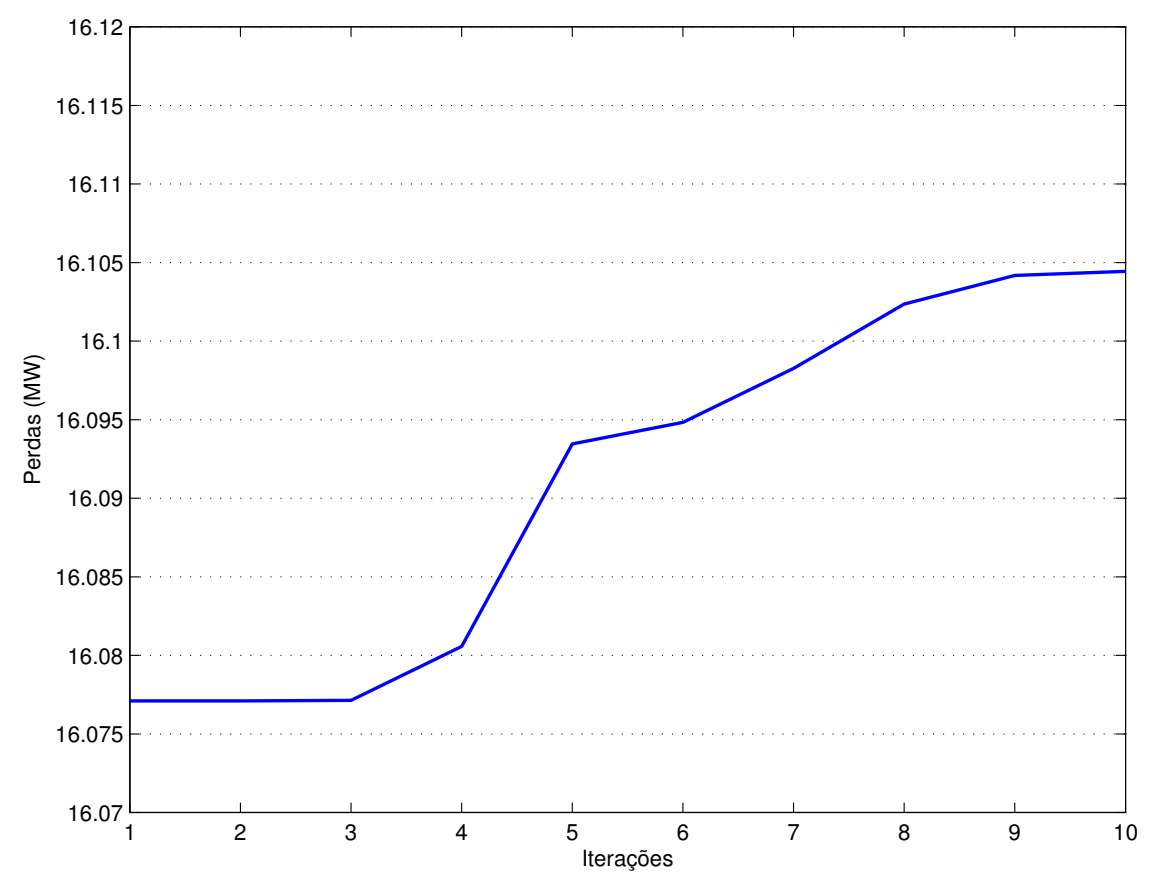

Figura 10.10 - Perdas ativas no sistema IEEE de 30 barras ao longo do processo de resolução do FPOR (9.4).

\subsubsection{Modelo com Variáveis de Controle Discretas e Restrições de Atuação de Dispositivos de Controle de Tensão}

Os limites da magnitude de tensão das barras controladas pelos transformadores e pelos bancos de capacitores do sistema IEEE de 30 barras são dados na Tabela 10.6. Observe que a magnitude de tensão da barra 10 possui dois dispositivos de controle: um transformador e um banco de capacitores.

De acordo com o algoritmo da FLBMP-discreto aplicado à resolução de (9.5) para o sistema IEEE de 30 barras:

- os valores iniciais dos parâmetros $\gamma$ das funções $\psi$ associadas aos taps dos transformadores e aos shunts variáveis foram $10^{-5}$;

- a constante $\beta$ foi considerada igual a 1 ;

- o fator $\tau$ de ajuste de $\gamma$ foi fixado em 10;

- e a tolerância $\xi$ para convergência do algoritmo da FLBMP-discreto foi de $10^{-4}$. 
Tabela 10.9 - Valores ótimos das variáveis de controle do sistema IEEE de 30 barras determinados pelo modelo (9.5).

\begin{tabular}{|c|c|c|c|c|}
\hline $\begin{array}{c}\text { Barra } \\
(\mathrm{k})\end{array}$ & $\begin{array}{c}V_{k} \\
\text { (p.u.) }\end{array}$ & $\begin{array}{c}b_{k}^{s h} \\
\text { (p.u.) }\end{array}$ & $\begin{array}{c}b_{a_{k}} \\
\text { (p.u.) }\end{array}$ & $\begin{array}{c}b_{b_{k}} \\
\text { (p.u.) }\end{array}$ \\
\hline 1 & 1,100 & - & - & - \\
\hline 2 & 1,076 & - & - & - \\
\hline 5 & 1,039 & - & - & - \\
\hline 8 & 1,037 & - & - & - \\
\hline 10 & - & 0,190 & 0,000 & 0,000 \\
\hline 11 & 1,094 & - & - & - \\
\hline 13 & 1,076 & - & - & - \\
\hline 24 & - & 0,000 & 0,000 & 0,040 \\
\hline $\begin{array}{c}\text { Da Barra } \\
(\mathrm{k})\end{array}$ & $\begin{array}{c}\text { Para Barra } \\
(\mathrm{m})\end{array}$ & $\begin{array}{c}t_{k m} \\
\text { (p.u.) }\end{array}$ & $\begin{array}{l}t_{a_{k m}} \\
\text { (p.u.) }\end{array}$ & $\begin{array}{l}t_{b_{k m}} \\
\text { (p.u.) }\end{array}$ \\
\hline 6 & 9 & 0,9800 & 0,0000 & 0,0000 \\
\hline 6 & 10 & 0,9700 & 0,0000 & 0,0000 \\
\hline 4 & 12 & 0,9300 & 0,0000 & 0,0000 \\
\hline 28 & 27 & 0,9800 & 0,0000 & 0,0100 \\
\hline \multicolumn{5}{|c|}{ Número de iterações $=6$} \\
\hline \multicolumn{5}{|c|}{ Perdas $=16,5273 \mathrm{MW}$} \\
\hline
\end{tabular}

Os valores iniciais considerados para as variáveis de controle discretas do sistema IEEE de 30 barras no caso base foram: $t_{6-9_{o}}=0,980$ p.u.; $t_{6-10_{o}}=0,970$ p.u.; $t_{4-12_{o}}=0,930$ p.u.; $t_{28-27_{o}}=0,970$ p.u.; $b_{10_{o}}^{s h}=0,190$ p.u. e $b_{24_{o}}^{s h}=0,040$ p.u.

Os valores ótimos das variáveis de controle determinados pela resolução do problema (9.5) para o sistema IEEE de 30 barras são apresentados na Tabela 10.9. Pela aplicação deste modelo, as perdas ativas neste sistema de transmissão foram reduzidas em 5,84\%, mantendo-se um perfil de tensão médio em torno de 1,0474 p.u.

As Figuras 10.11, 10.12 e 10.13 mostram, respectivamente, os valores das susceptâncias equivalentes dos shunts das barras 10 e 24, dos taps dos transformadores e da função perdas ao longo do processo iterativo de resolução do problema (9.5) pelo algoritmo da FLBMP-discreto. A Figura 10.14 mostra os perfis das magnitudes de tensão dos pontos ótimos determinados por cada FPOR. 


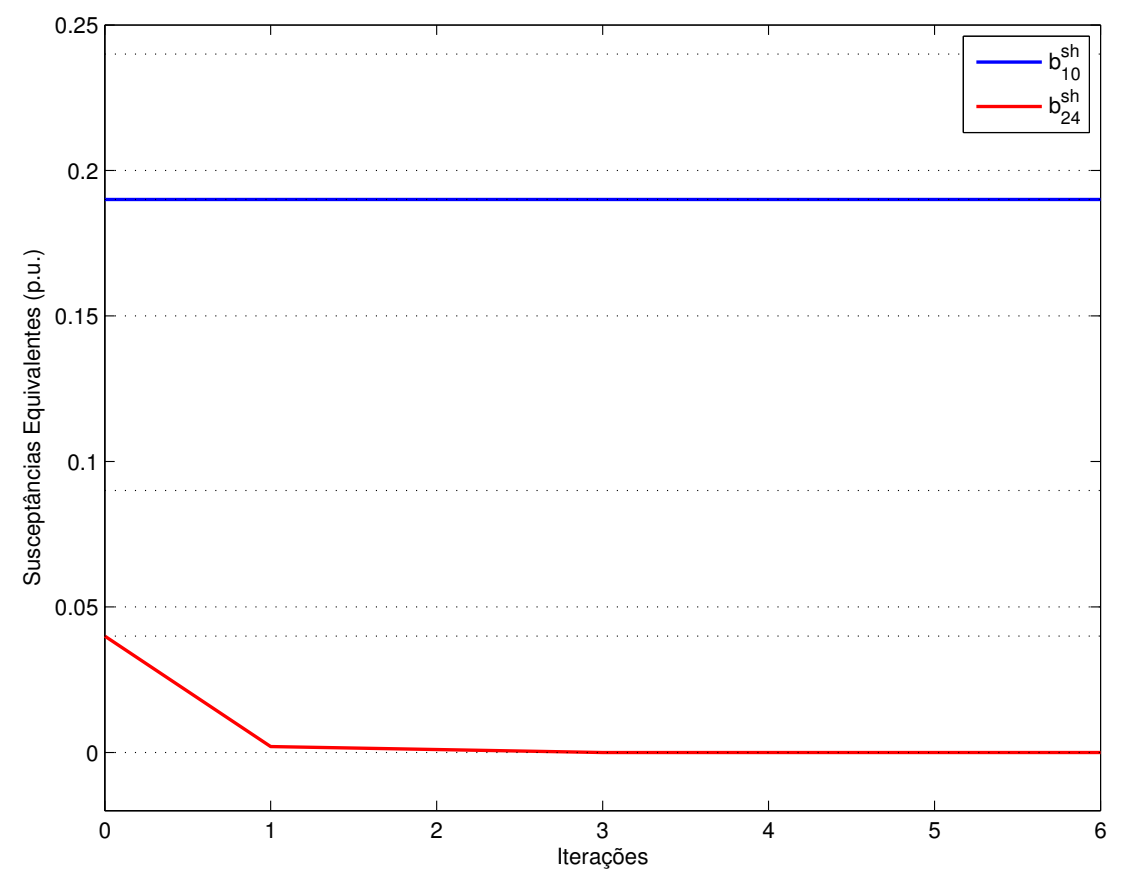

Figura 10.11 - Valores das susceptâncias equivalentes dos bancos de capacitores do sistema IEEE de 30 barras ao longo do processo de resolução do FPOR (9.5).

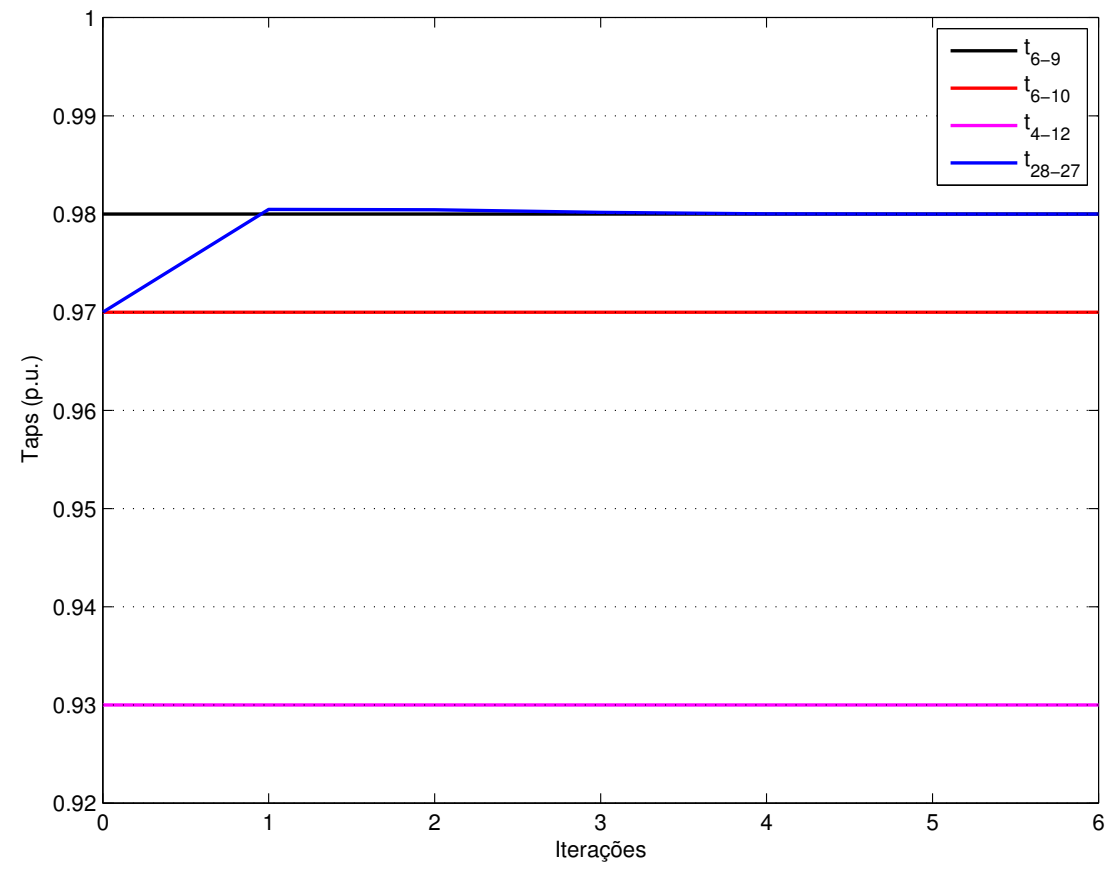

Figura 10.12 - Valores dos taps dos transformadores do sistema IEEE de 30 barras ao longo do processo de resolução do modelo (9.5). 


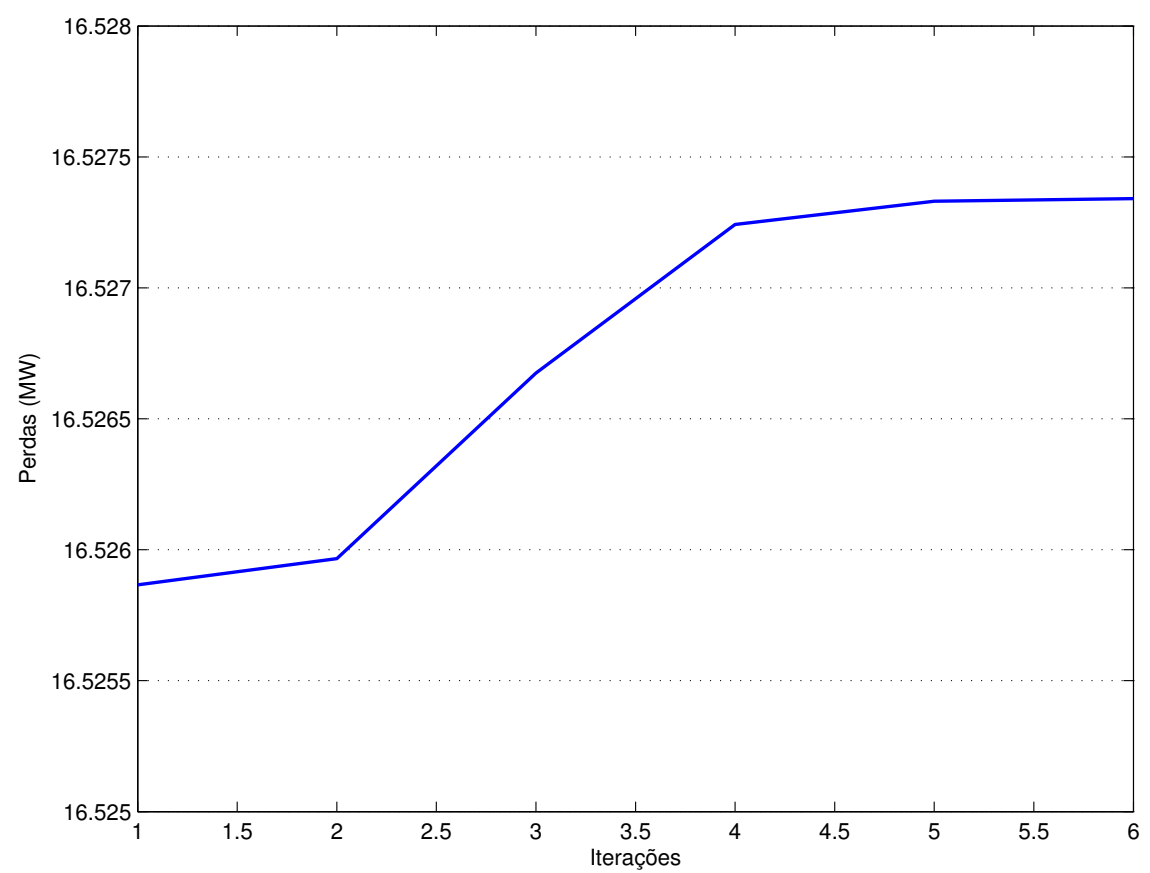

Figura 10.13 - Perdas ativas no sistema IEEE de 30 barras ao longo do processo de resolução do modelo (9.5).

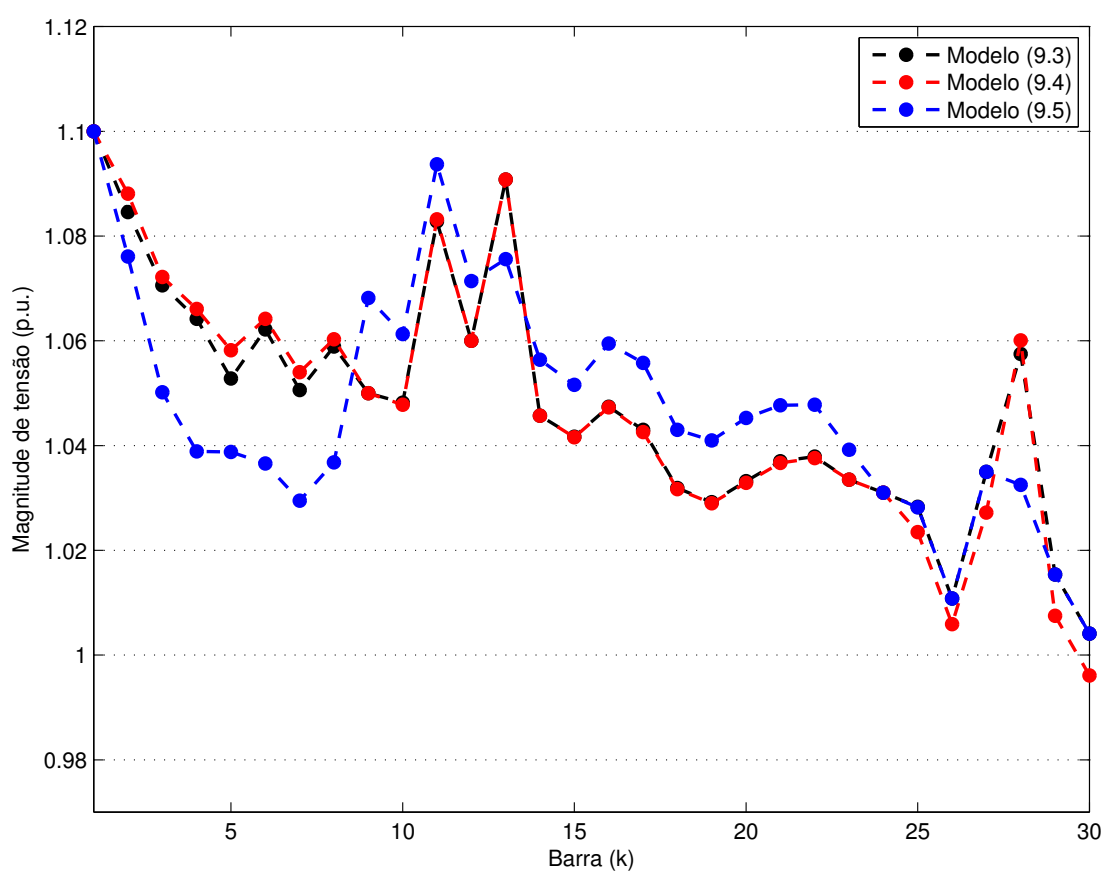

Figura 10.14 - Perfis das magnitudes de tensão determinados pelos modelos (9.3), (9.4) e (9.5) para o sistema IEEE de 30 barras. 


\subsection{Sistema IEEE de 57 Barras}

O sistema IEEE de 57 barras possui as seguintes características:

- 1 barra slack;

- 6 barras com controle de geração de reativos;

- 50 barras de carga;

- 3 bancos de capacitores;

- 80 linhas de transmissão;

- 20 transformadores com tap variável.

Para esse sistema, considerou-se que os limites mínimo e máximo da magnitude de tensão fossem, respectivamente, 0,950 e 1,100 p.u.

Na modelagem das variáveis de controle discretas, considerou-se que o tamanho do passo entre dois valores consecutivos dos taps dos transformadores fosse 0,01 p.u. e que seus limites mínimo e máximo fossem 0,880 e 1,120 p.u. Por fim, considerou-se que os valores discretos que as susceptâncias equivalentes dos shunts das barras 18, 25 e 53 podem assumir na solução do problema são dados por:

$$
\begin{aligned}
& b_{18}^{s h} \in\{0 ; 0,05 ; 0,10 ; 0,12 ; 0,15 ; 0,17 ; 0,22 ; 0,27\} \\
& b_{25}^{s h} \in\{0 ; 0,02 ; 0,03 ; 0,04 ; 0,05 ; 0,06 ; 0,07 ; 0,09\} \\
& b_{53}^{s h} \in\{0 ; 0,065 ; 0,10 ; 0,165\}
\end{aligned}
$$

Pela aplicação dos modelos (9.3), (9.4) e (9.5), os valores ótimos das susceptâncias equivalentes dos shunts e das perdas são apresentados na Tabela 10.10.

No modelo (9.5), as susceptâncias equivalentes dos shunts das barras 18, 25 e 53 não precisaram ser ajustadas para controlar a magnitude de tensão da barra à qual estão conectados. As Figuras 10.15, 10.16 e 10.17 mostram, respectivamente, os valores dos taps dos transformadores determinados pelos modelos (9.3), (9.4) e (9.5). Na Figura 10.17, os 
Tabela 10.10 - Valores ótimos das susceptâncias equivalentes dos shunts do sistema IEEE de 57 barras determinados pelos modelos (9.3), (9.4) e (9.5).

\begin{tabular}{cccc}
\hline & Modelo (9.3) & Modelo (9.4) & Modelo (9.5) \\
\hline$b_{18}^{\text {sh }}$ (p.u.) & 0,2700 & 0,2700 & 0,1000 \\
$b_{25}^{\text {sh }}$ (p.u.) & 0,0900 & 0,0900 & 0,0600 \\
$b_{53}^{\text {sh }}$ (p.u.) & 0,1198 & 0,1000 & 0,0650 \\
\hline Perdas (MW) & 17,9120 & 17,9910 & 18,5917 \\
\hline Iterações & - & 14 & 30 \\
Tolerância $\xi$ & - & $10^{-4}$ & $10^{-3}$ \\
\hline
\end{tabular}

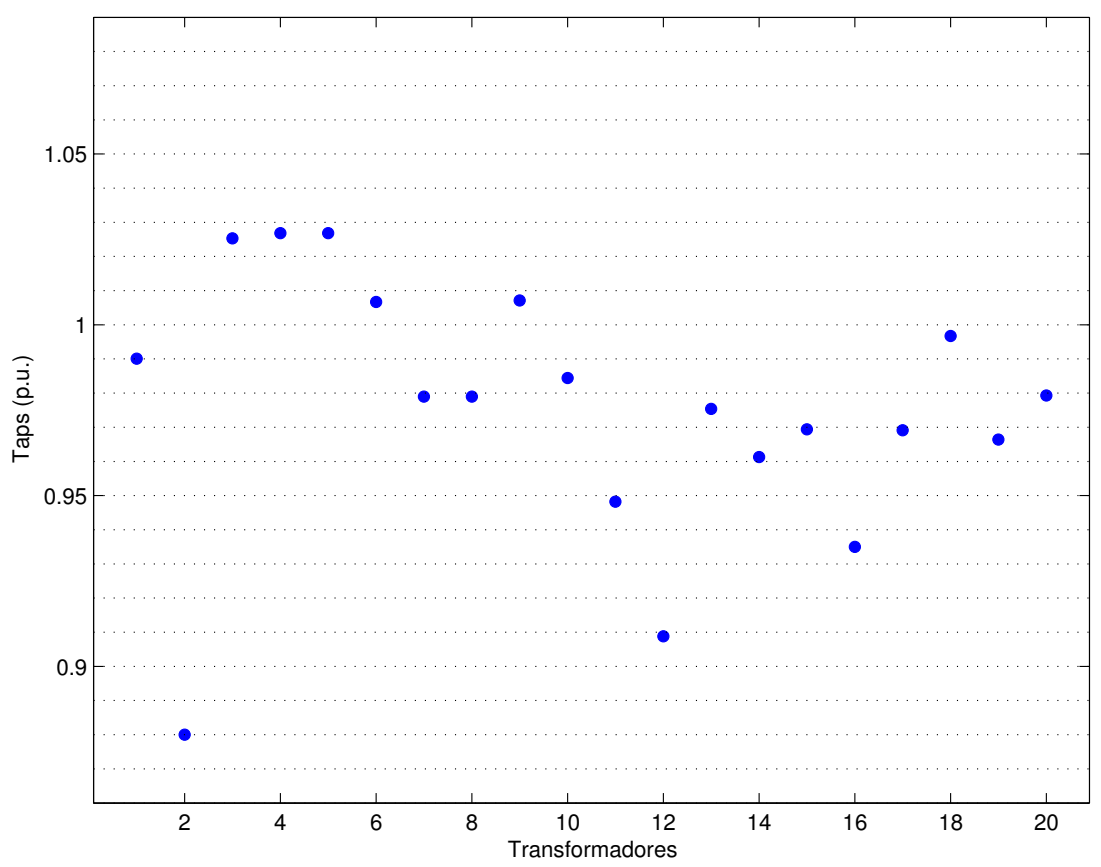

Figura 10.15 - Valores ótimos dos taps dos transformadores determinados pelo modelo (9.3) para o sistema IEEE de 57 barras.

pontos em azul correspondem aos taps dos transformadores que foram ajustados para controlar a magnitude de tensão de uma de suas barras terminais e os pontos em vermelho correspondem aos taps dos transformadores que não precisaram ser ajustados. 


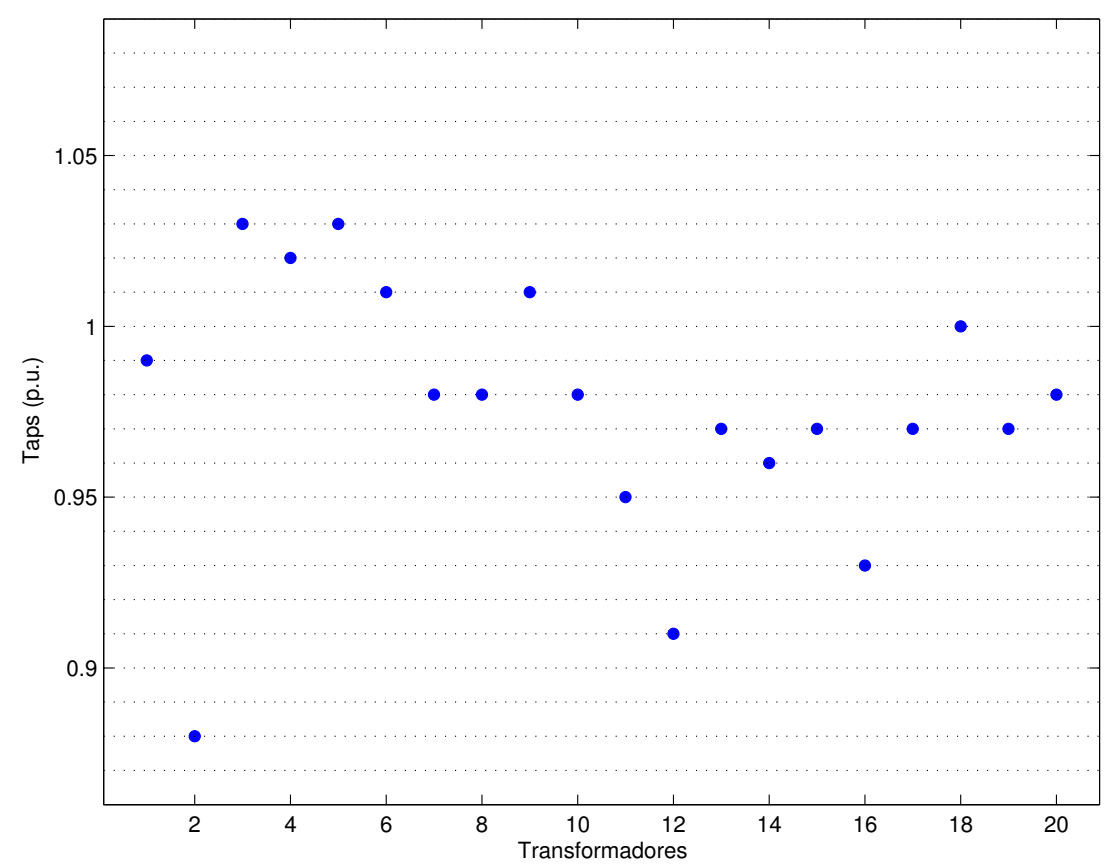

Figura 10.16 - Valores ótimos dos taps dos transformadores determinados pelo modelo (9.4) para o sistema IEEE de 57 barras.

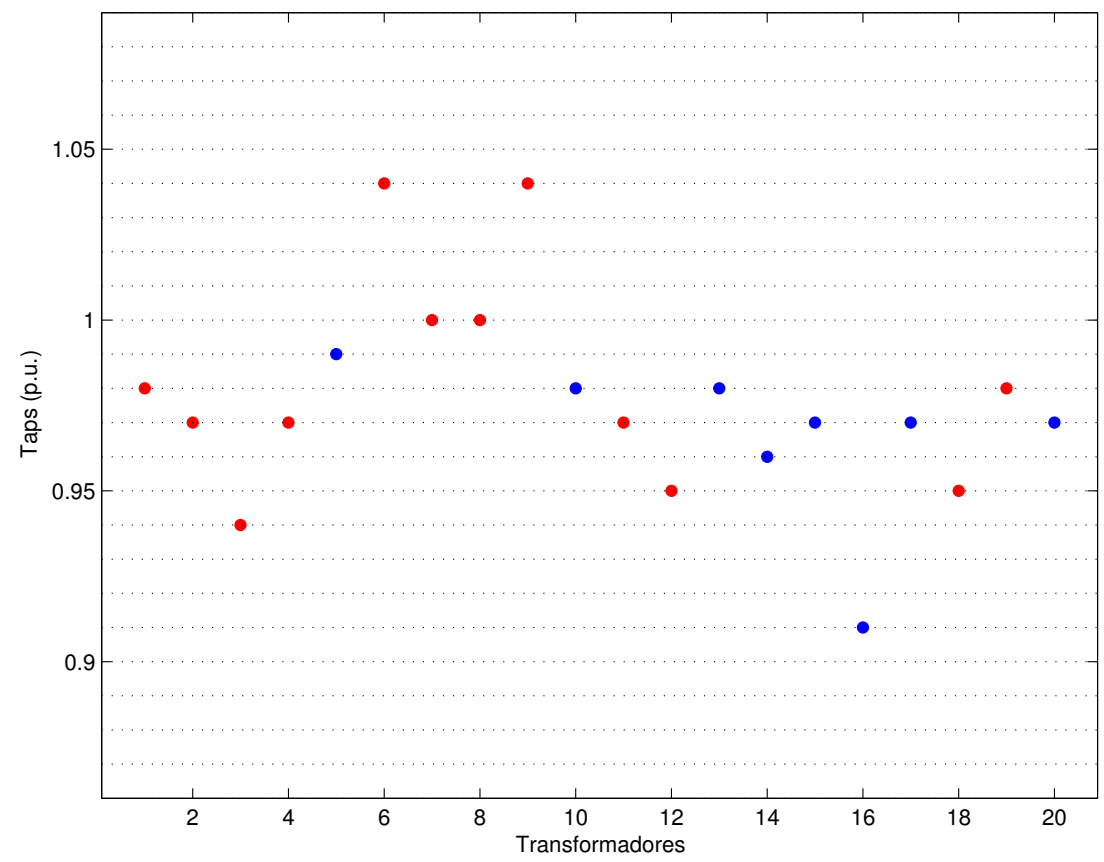

Figura 10.17 - Valores ótimos dos taps dos transformadores determinados pelo modelo (9.5) para o sistema IEEE de 57 barras. 


\subsection{Sistema IEEE de 118 Barras}

O sistema IEEE de 118 barras possui as seguintes características:

- 1 barra slack;

- 53 barras com controle de geração de reativos;

- 64 barras de carga;

- 14 shunts variáveis;

- 172 linhas de transmissão;

- 9 transformadores com tap variável.

Para esse sistema, considerou-se que os limites mínimo e máximo da magnitude de tensão fossem, respectivamente, 0,950 e 1,100 p.u.

Na modelagem das variáveis de controle discretas, considerou-se que o tamanho do passo entre dois valores consecutivos dos taps dos transformadores fosse 0,0075 p.u. e que seus limites mínimo e máximo fossem 0,880 e 1,120 p.u.

A potência dos shunts determina o quão preciso pode ser o controle. Os bancos de capacitores, em particular, podem quase sempre ser chaveados em unidades menores, enquanto reatores shunt, devido aos altos custos, são mais frequentemente instalados em uma única unidade (GÓMEZ-EXPÓSITO; CONEJO; CAÑIZARES, 2011). Dessa forma, considerou-se que os valores discretos que as susceptâncias equivalentes dos shunts podem 
assumir na solução do problema são dados por:

$$
\begin{aligned}
b_{5}^{s h} & \in\{0 ;-0,40\} \\
b_{34}^{s h} & \in\{0 ; 0,06 ; 0,07 ; 0,13 ; 0,14 ; 0,20\} \\
b_{37}^{s h} & \in\{0 ;-0,25\} \\
b_{44}^{s h} & \in\{0 ; 0,10\} \\
b_{45}^{s h} & \in\{0 ; 0,10\} \\
b_{46}^{s h} & \in\{0 ; 0,10\} \\
b_{48}^{s h} & \in\{0 ; 0,15\} \\
b_{74}^{s h} & \in\{0 ; 0,08 ; 0,12 ; 0,20\} \\
b_{79}^{s h} & \in\{0 ; 0,10 ; 0,20\} \\
b_{82}^{s h} & \in\{0 ; 0,10 ; 0,20\} \\
b_{83}^{s h} & \in\{0 ; 0,10 ; 0,20\} \\
b_{105}^{s h} & \in\{0 ; 0,10 ; 0,20\} \\
b_{107}^{s h} & \in\{0 ; 0,06 ; 0,07 ; 0,13 ; 0,14 ; 0,20\} \\
b_{110}^{s h} & \in\{0 ; 0,06 ; 0,07 ; 0,13 ; 0,14 ; 0,20\}
\end{aligned}
$$

Pela aplicação dos modelos (9.3), (9.4) e (9.5), as perdas ativas calculadas em função das soluções ótimas são dadas, respectivamente, por 106,4198 MW, 106, 4533 MW e 106,7127 MW. O algoritmo da FLBMP-discreto convergiu para uma solução de (9.4) e (9.5) em 6 e 7 iterações, respectivamente, com uma tolerância $\xi$ para convergência de $10^{-4}$.

As Figuras 10.18, 10.19, 10.20, 10.21, 10.22 e 10.23 mostram, respectivamente, os valores das susceptâncias equivalentes dos shunts e dos taps dos transformadores determinados pelos modelos (9.3), (9.4) e (9.5). Nas Figuras 10.22 e 10.23, os pontos em azul correspondem aos controles que foram ajustados e os pontos em vermelho correspondem aos controles que não precisaram ser ajustados. 


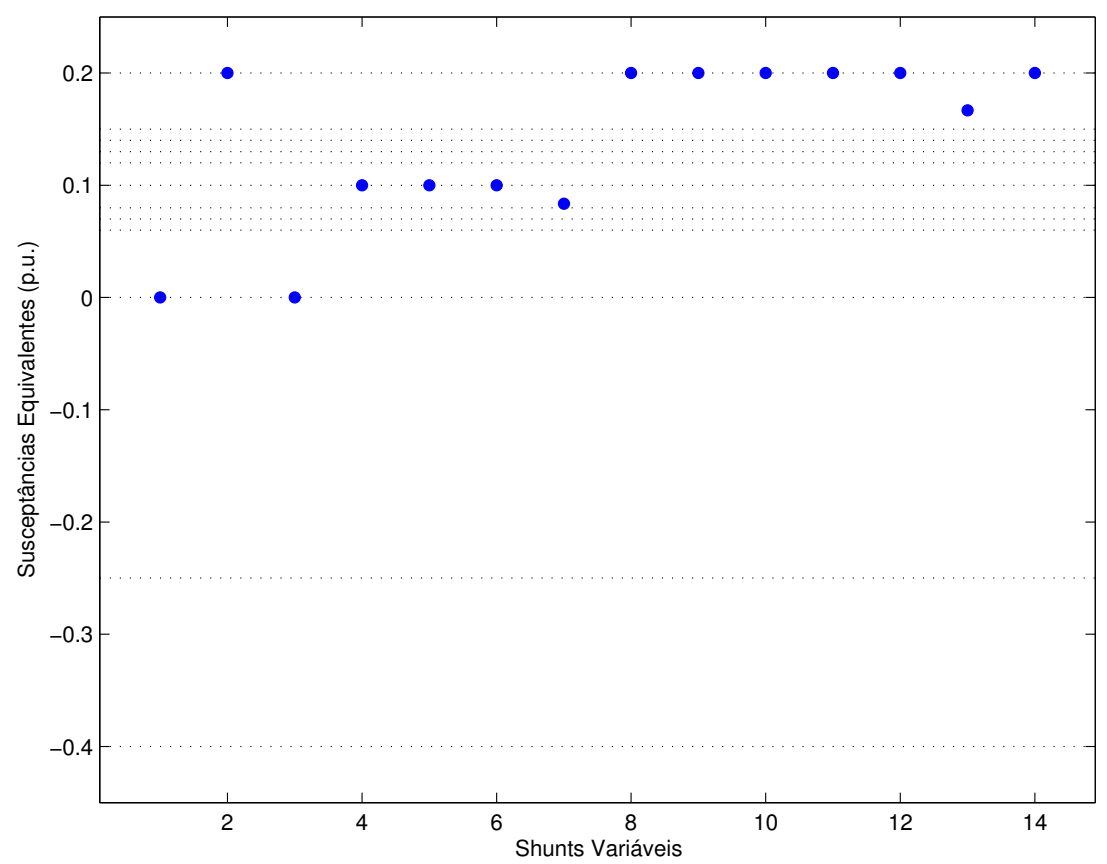

Figura 10.18 - Valores ótimos das susceptâncias equivalentes dos bancos de capacitores e reatores shunt determinados pelo modelo (9.3) para o sistema IEEE de 118 barras.

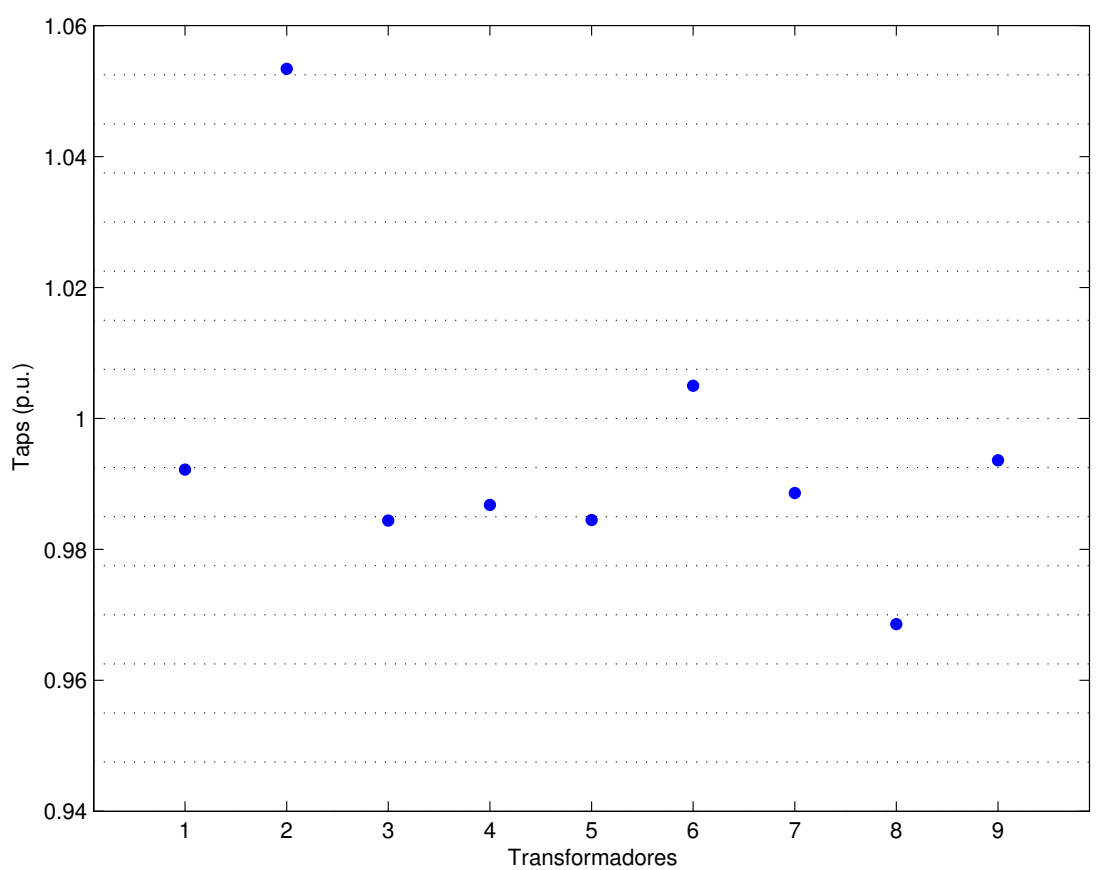

Figura 10.19 - Valores ótimos dos taps dos transformadores determinados pelo modelo (9.3) para o sistema IEEE de 118 barras. 


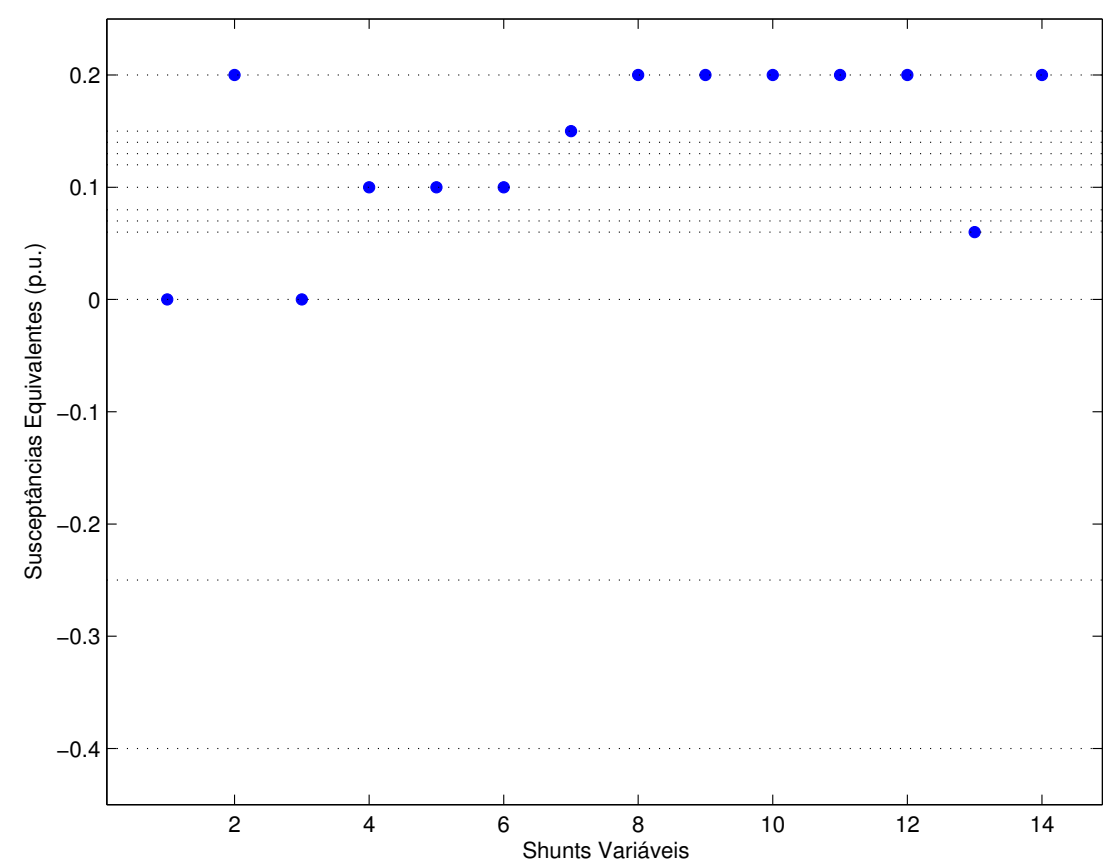

Figura 10.20 - Valores ótimos das susceptâncias equivalentes dos bancos de capacitores e reatores shunt determinados pelo modelo (9.4) para o sistema IEEE de 118 barras.

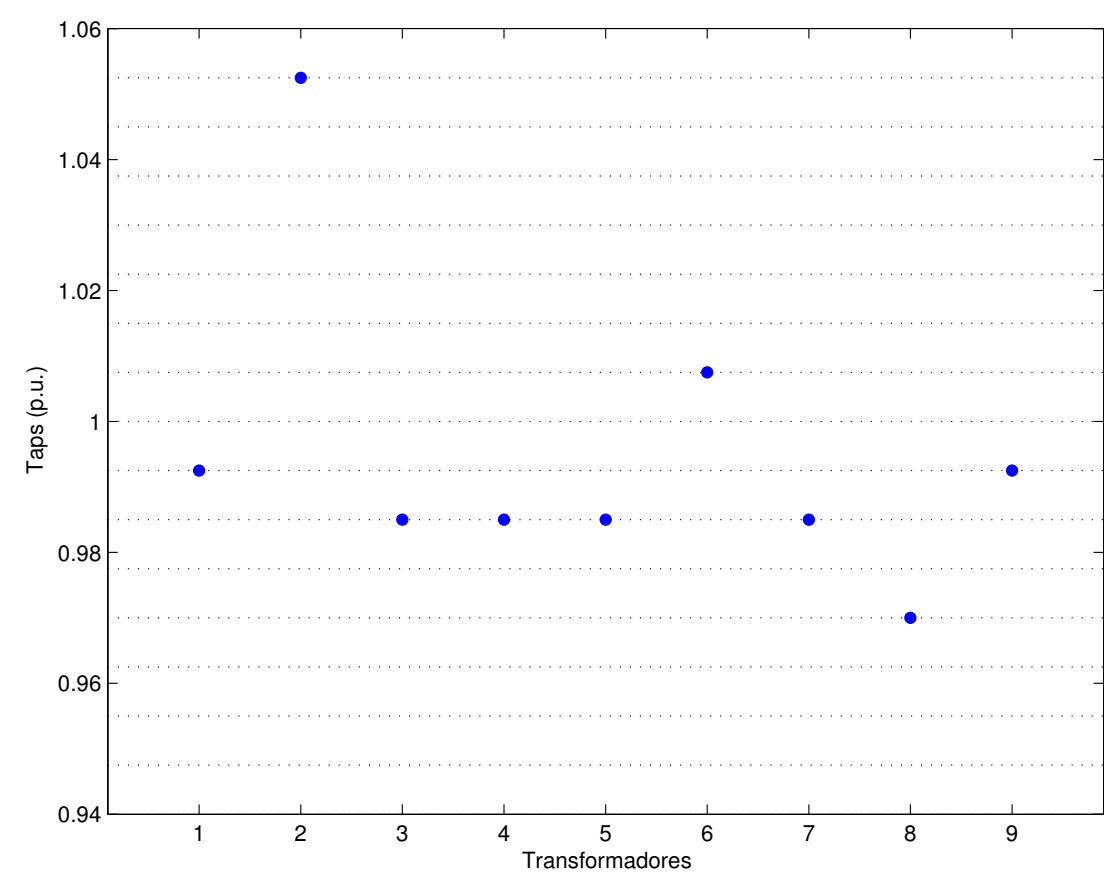

Figura 10.21 - Valores ótimos dos taps dos transformadores determinados pelo modelo (9.4) para o sistema IEEE de 118 barras. 


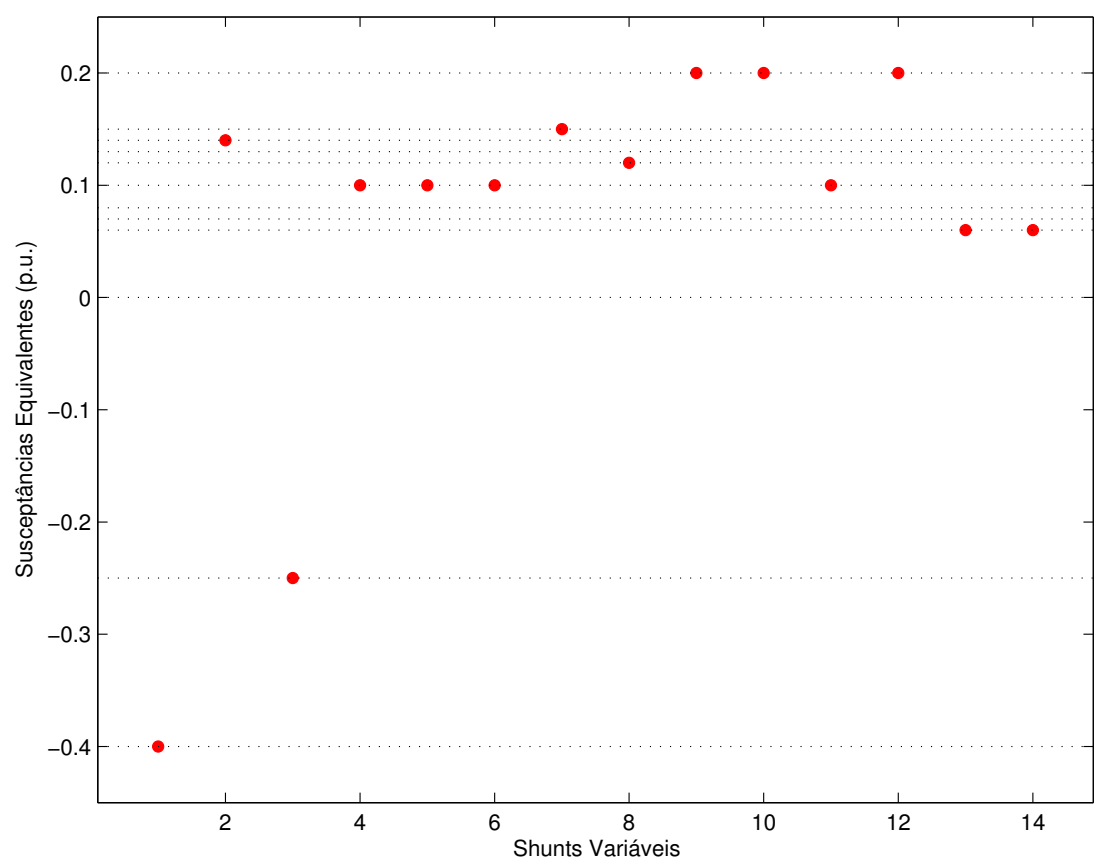

Figura 10.22 - Valores ótimos das susceptâncias equivalentes dos bancos de capacitores e reatores shunt determinados pelo modelo (9.5) para o sistema IEEE de 118 barras.

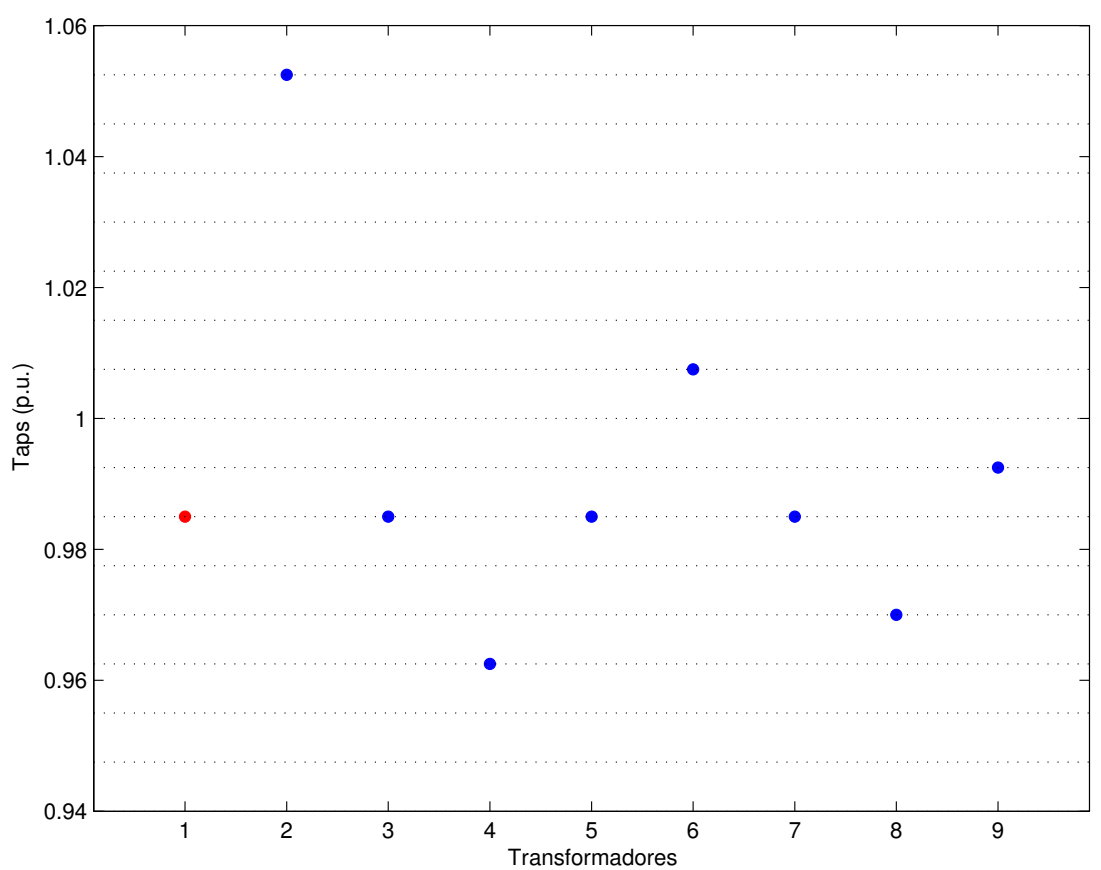

Figura 10.23 - Valores ótimos dos taps dos transformadores determinados pelo modelo (9.5) para o sistema IEEE de 118 barras. 


\subsection{Sistema Equivalente CESP $440 \mathrm{kV}$ de 53 Barras}

O sistema equivalente CESP de 53 barras possui as seguintes características:

- 1 barra slack;

- 8 barras com controle de geração de reativos;

- 44 barras de carga;

- 2 reatores shunt;

- 37 linhas de transmissão;

- 48 transformadores com tap variável.

Para esse sistema, considerou-se que os limites mínimo e máximo da magnitude de tensão fossem, respectivamente, 0,950 e 1,150 p.u.

Na modelagem das variáveis de controle discretas, considerou-se que o tamanho do passo entre dois valores consecutivos dos taps dos transformadores fosse 0,01 p.u. Por fim, considerou-se que valores discretos que as susceptâncias equivalentes dos reatores shunt conectados às barras 552 e 584 podem assumir na solução do problema são dados por:

$$
\begin{aligned}
& b_{552}^{s h} \in\{0 ;-0,90\} \\
& b_{584}^{s h} \in\{0 ;-0,90\}
\end{aligned}
$$

Pela aplicação dos modelos (9.3), (9.4) e (9.5), os valores ótimos das susceptâncias equivalentes dos shunts e das perdas são apresentados na Tabela 10.11.

No modelo (9.5), as susceptâncias equivalentes dos reatores shunt não precisaram ser ajustadas para controlar a magnitude de tensão da barra à qual estão conectados. As Figuras 10.24, 10.25 e 10.26 mostram, respectivamente, os valores dos taps dos transformadores determinados pelos modelos (9.3), (9.4) e (9.5). Na Figura 10.26, o ponto em azul corresponde ao tap do transformador que foi ajustado para controlar a magnitude de tensão de uma de suas barras terminais e os pontos em vermelho correspondem aos taps dos transformadores que não precisaram ser ajustados. 
Tabela 10.11 - Valores das susceptâncias equivalentes dos reatores shunt e perdas no sistema equivalente CESP $440 \mathrm{kV}$ de 53 barras.

\begin{tabular}{cccc}
\hline & Modelo (9.3) & Modelo (9.4) & Modelo (9.5) \\
\hline$b_{552}^{\text {sh }}$ (p.u.) & 0,0000 & 0,0000 & $-0,9000$ \\
$b_{584}^{\text {sh }}$ (p.u.) & 0,0000 & 0,0000 & $-0,9000$ \\
\hline Perdas (MW) & 196,6252 & 197,6372 & 211,3992 \\
\hline Iterações & - & 10 & 9 \\
Tolerância $\xi$ & - & $10^{-4}$ & $10^{-4}$ \\
\hline
\end{tabular}

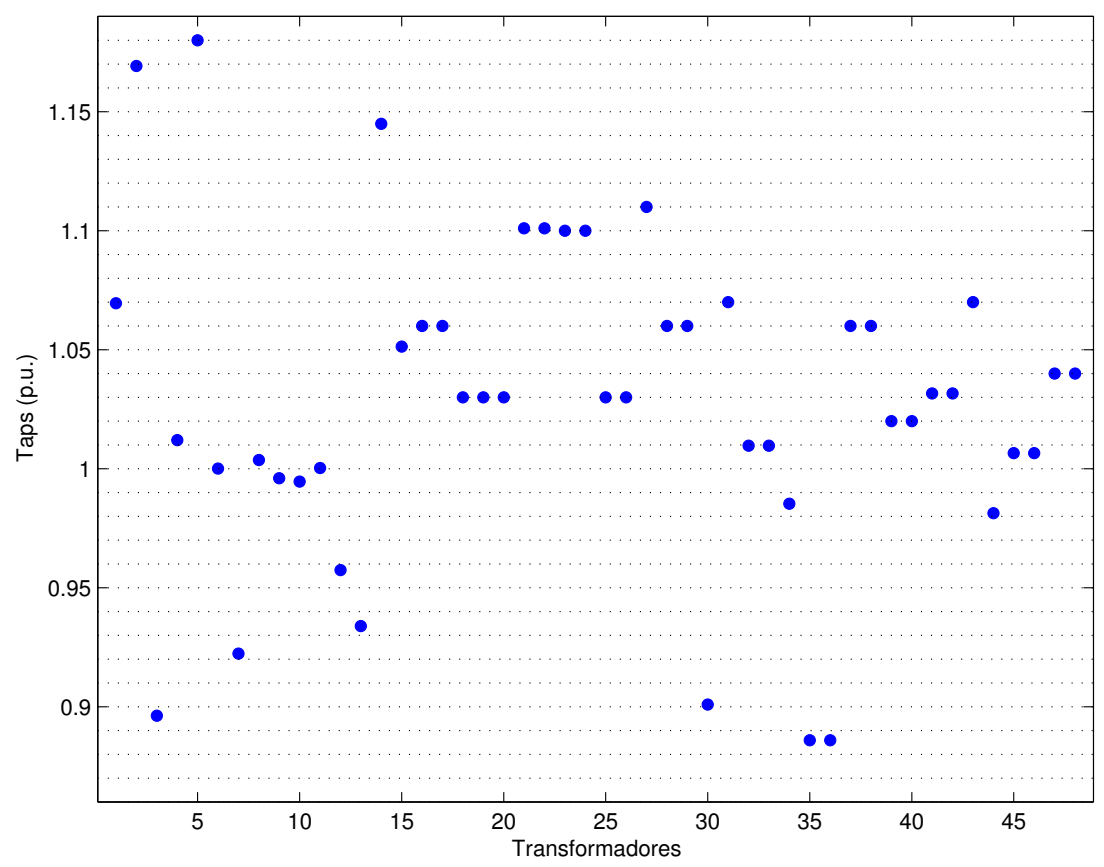

Figura 10.24 - Valores ótimos dos taps dos transformadores determinados pelo modelo (9.3) para o sistema equivalente CESP $440 \mathrm{kV}$ de 53 barras. 


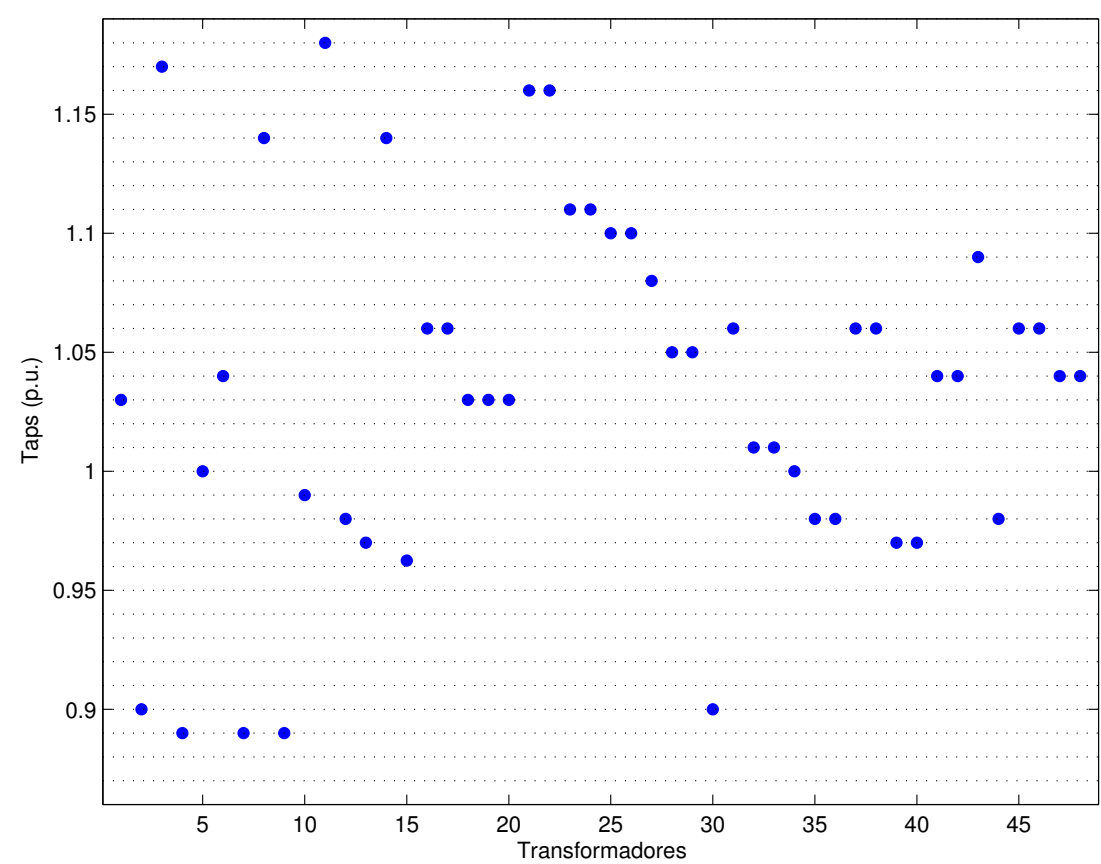

Figura 10.25 - Valores ótimos dos taps dos transformadores determinados pelo modelo (9.4) para o sistema equivalente CESP $440 \mathrm{kV}$ de 53 barras.

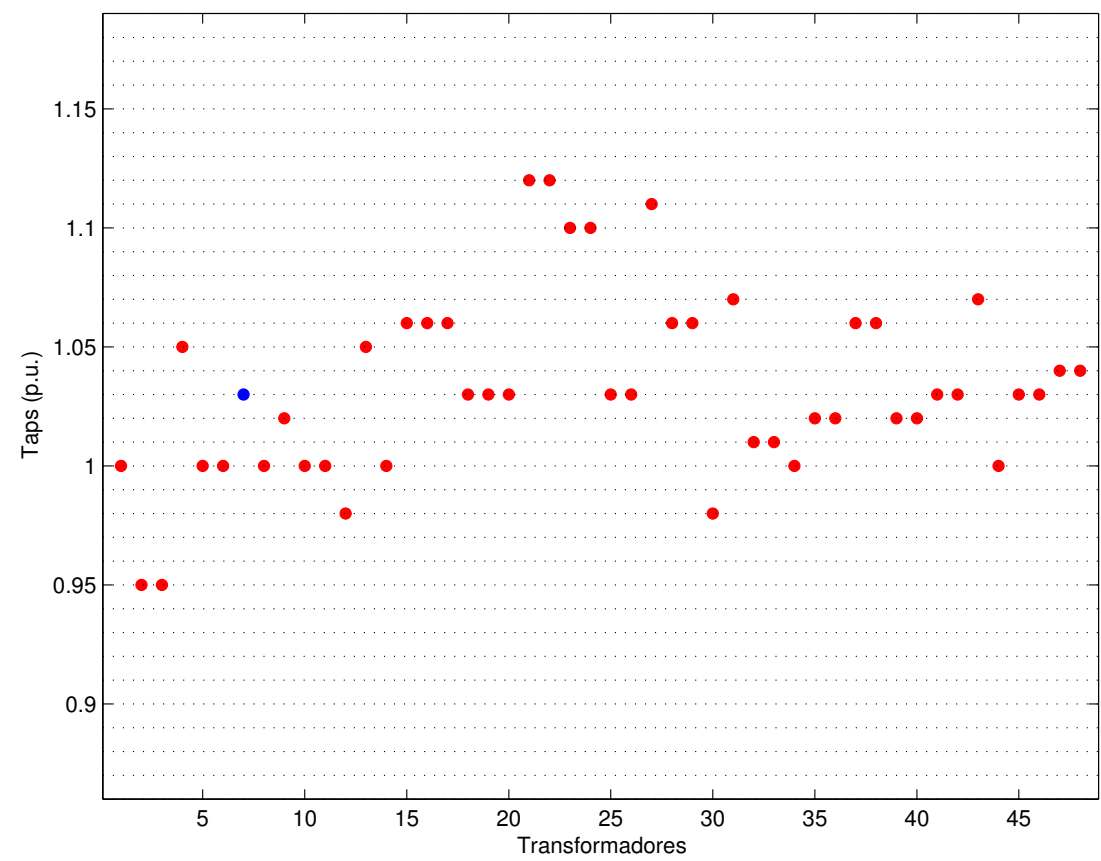

Figura 10.26 - Valores ótimos dos taps dos transformadores determinados pelo modelo (9.5) para o sistema equivalente CESP $440 \mathrm{kV}$ de 53 barras. 


\section{Capítulo 11}

\section{Conclusões}

O FPO é formulado como um problema de PNL, estático, não convexo, restrito, de grande porte e com variáveis contínuas e discretas, cujas proposições de novos modelos acompanham de perto o desenvolvimento de técnicas robustas e eficientes para sua resolução. Historicamente, problemas de FPO são resolvidos por métodos clássicos de PNL, ou uma associação destes, considerando as variáveis de controle discretas como contínuas e "utilizando" todos controles na determinação do desempenho operacional ótimo do sistema. Essas abordagens, no entanto, estão muito longe da realidade de um SEE, pois alguns de seus controles só podem ser ajustados por passos discretos, e a atuação irrestrita de dispositivos de controle é impraticável à operação de sistemas de grande porte. Nesse contexto, este trabalho propôs um novo modelo e uma abordagem para resolução do problema de minimização das perdas ativas na transmissão, com variáveis de controle discretas e restrições de atuação de dispositivos de controle de tensão.

Matematicamente, o problema de FPOR para minimização das perdas ativas na transmissão foi formulado como um problema de PNL com variáveis contínuas e discretas e restrições de complementaridade, cuja abordagem para resolução se baseia na resolução de uma sequência de problemas pelo algoritmo da FLBMP-discreto. Nessa abordagem, o problema de FPOR é modificado da seguinte forma: 1) as variáveis discretas 
são tratadas como contínuas pelas funções $\psi ; 2)$ as restrições de complementaridade são transformadas em restrições de desigualdade equivalentes; e 3) as restrições de desigualdade são transformadas em restrições de igualdade a partir do acréscimo de variáveis de folga não negativas.

Para resolver o problema modificado, a condição de não negatividade das variáveis de folga é tratada por uma FBMEQ. O problema modificado é transformado em um problema Lagrangiano pelo método da FLBMP, cuja solução é determinada a partir da aplicação das condições necessárias de otimalidade. No algoritmo da FLBMP-discreto, uma sequência de problemas é resolvida pelo método da FLBMP até que todas as variáveis do problema modificado associadas às variáveis discretas do problema original assumam valores discretos.

A presença de variáveis de controle discretas em conjunto com a função objetivo, restrições não lineares e restrições de complementaridade do modelo proposto dificultam a determinação da sua solução. Problemas de FPOR como o proposto neste trabalho possuem características de problemas de PNL e de PIM, além de possuírem características próprias que não aparecem nesses dois tipos de problema.

Os testes realizados demonstraram a eficácia do modelo proposto e a robustez da abordagem para resolução de problemas de FPOR desenvolvida. Além de considerar a modelagem de variáveis de controle discretas, o modelo proposto restringe a atuação de dispositivos de controle de tensão através de sua representação por problemas de complementaridade.

As principais contribuições deste trabalho podem ser resumidas da seguinte forma: do ponto de vista matemático, contribuiu-se com o desenvolvimento de uma nova abordagem para resolução de problemas de PNL com variáveis contínuas e discretas e restrições de complementaridade; do ponto de vista operacional, foram desenvolvidos um novo modelo e uma nova abordagem do tipo primal-dual de métodos aplicados à resolução de problemas de FPOR, os quais são de grande interesse em estudos de planejamento e operação de SEEs. 


\subsection{Trabalhos Futuros}

As perspectivas de continuidade deste trabalho são as seguintes:

- verificação da influência de problemas de complementaridade na trajetória de convergência do método da FLBMP;

- proposição de regras para o ajuste adaptativo do parâmetro $\gamma$ da função $\psi$ associada às variáveis de controle discretas do problema de FPOR;

- aplicação da abordagem desenvolvida na resolução de problemas de otimização de dois níveis;

- determinação de regras de inicialização dos multiplicadores de Lagrange associados à FBMEQ;

- comparação do desempenho da abordagem de resolução desenvolvida com o de outras abordagens propostas na literatura;

- realização de testes com sistemas de maior porte;

- aplicação da abordagem de resolução desenvolvida a outros problemas de análise de SEEs. 


\section{Referências Bibliográficas}

AFFOnSO, C. M.; SILVA, L. J. da; SILVA, L. C. P. da. Avaliação da estabilidade de tensão do sistema brasileiro - um caso de estudo. Revista Controle \& Automação, v. 19, n. 3, p. 327-336, jul., ago. e set. 2008.

AGÊNCIA NACIONAL DE ENERGIA ELÉTRICA (ANEEL). Atlas de energia elétrica do Brasil. 3. ed. Brasília: ANEEL, 2008. 236 p.

ALMEIDA, K. C.; SENNA, F. S. Optimal active-reactive power dispatch under competition via bilevel programming. IEEE Transactions on Power Systems, v. 26, n. 4, p. 2345-2354, Nov. 2011.

BAKIRTZIS, A. G.; BISKAS, P. N.; ZOUMAS, C. E.; PETRIDIS, V. Optimal power flow by enhanced genetic algorithm. IEEE Transactions on Power Systems, v. 17, n. 2, p. 229-236, May 2002.

BAPTISTA, E. C. Método da função Lagrangiana aumentada-barreira logarítmica para a solução do problema de fluxo de potência ótimo. Tese (Doutorado) - Departamento de Engenharia Elétrica, Escola de Engenharia de São Carlos, Universidade de São Paulo, 2001.

BAPtiStA, E. C.; BELATI, E. A.; SOUSA, V. A. de; COSTA, G. R. M. da. Primal-dual logarithmic barrier and augmented Lagrangian function to the loss minimization in power systems. Electric Power Components and Systems, v. 34, n. 7, p. 775-784, 2006. 
BAUTISTA, G.; ANJOS, M. F.; VANNELLI, A. Numerical study of affine supply function equilibrium in AC network-constrained markets. IEEE Transactions on Power Systems, v. 22, n. 3, p. 1174-1184, Aug. 2007.

BAZARAA, M. S. Nonlinear programming: theory and algorithms. 2nd. ed. [S.l.]: John Wiley \& Sons, Inc., 1993.

BENSON, H. Y.; SHANNO, D. F.; VANDERBEI, R. J. Interior-point methods for nonconvex nonlinear programming: complementarity constraints. Technical Report, Operations Research and Financial Engineering, Princeton University, Princeton, v. 2, n. 2, p. 1-20, Sept. 2002.

BRASIL. Ministério de Minas e Energia (MME). Monitoramento do sistema elétrico brasileiro: boletim de outubro de 2011. Brasília: MME, 2011. 33 p.

Monitoramento do sistema elétrico brasileiro: boletim de setembro de 2012. Brasília: MME, 2012. 35 p.

. Monitoramento do sistema elétrico brasileiro: boletim de abril de 2012. Brasília: MME, 2012. $35 \mathrm{p}$.

BREITFELD, M. G.; SHANNO, D. F. A globally convergent penalty-barrier algorithm for nonlinear programming and its computational performance. Rutcor Research Report (RRR) 12-94, p. 1-39, Apr. 1994.

- Computational experience with penalty-barrier methods for nonlinear programming. Annals of Operations Research, v. 62, n. 1, p. 439-463, 1996.

CÂMARA DE COMERCIALIZAÇÃO DE ENERGIA ELÉTRICA (CCEE). 2012. Disponível em: <http://www.ccee.org.br>. Acesso em: 8 dez. 2012.

CAPITANESCU, F.; WEHENKEL, L. Sensitivity-based approaches for handling discrete variables in optimal power flow computations. IEEE Transactions on Power Systems, v. 25, n. 4, p. 1780-1789, Nov. 2010. 
CARPENTIER, J. L. Contribution à l'étude du dispatching économique. Bulletin de la Société Française des Electriciens, v. 3, n. 8, p. 431-447, 1962.

CARROL, C. W. The created response surface technique for optimizing nonlinear, restrained systems. Operations Research, v. 9, n. 2, p. 169-184, Mar. 1961.

CENTRO DE PESQUiSAS DE ENERGIA ELÉTRICA (CEPEL). Programa de Análise de Redes V08-Ago04: Manual do usuário. Rio de Janeiro: CEPEL, 2004. 229 p.

CHEN, T. W. C.; VASSILIADIS, V. S. Solution of general nonlinear optimization problems using the penalty/modified barrier method with the use of exact Hessians. Computers $\&$ Chemical Engineering, v. 27, n. 4, p. 501-525, Apr. 2003.

COSTA, G. R. M. da; COSTA, C. E. U.; SOUZA, A. M. Comparative studies of optimization methods for the optimal power flow problem. Electric Power Systems Research, v. 56, n. 3, p. 249-254, Nov. 2000.

DING, X.; WANG, X.; SONG, Y. H. Interior point cutting plane method for optimal power flow. IMA Journal of Management Mathematics, v. 15, n. 4, p. 355-368, 2004.

DOMMEL, H. W.; TINNEY, W. F. Optimal power flow solutions. IEEE Transactions on Power Apparatus and Systems, PAS-87, n. 10, p. 1866-1876, Oct. 1968.

FERRIS, M. C.; PANG, J. S. Engineering and economic applications of complementarity problems. SIAM Review, v. 39, n. 4, p. 669-713, Dec. 1997.

FIACCO, A. V.; McCORMICK, G. P. Nonlinear programming: sequential unconstrained minimization techniques. [S.l.]: Wiley, 1968.

FOURER, R.; GAY, D. M.; KERNIGHAN, B. W. AMPL: A modeling language for mathematical programming. 2nd. ed. [S.1.]: Duxbury Press, 2002. 540 p.

FRISCH, K. R. The logarithmic potential method of convex programming. Memorandum, Institute of Economics, University of Oslo, Oslo, May 1955. 
GÓmeZ-EXPÓSITO, A.; CONEJO, A. J.; CAÑIZARES, C. A. Sistemas de energia elétrica: análise e operação. Tradução e revisão técnica Antonio Padilha Feltrin, José Roberto Sanches Mantovani, Rubén Romero. Rio de Janeiro: LTC, 2011. 554 p.

GRANVILLE, S. Optimal reactive dispatch through interior point methods. IEEE Transactions on Power Systems, v. 9, n. 1, p. 136-146, Feb. 1994.

GROUP, W. Common format for exchange of solved load flow data. IEEE Transactions on Power Apparatus and Systems, PAS-92, n. 6, p. 1916-1925, Nov. 1973.

HUNEAULT, M.; GALIANA, F. D. A survey of the optimal power flow literature. IEEE Transactions on Power Systems, v. 6, n. 2, p. 762-770, May 1991.

INTERIOR Point Optimizer (IPOPT), version 3.10, release 3.10.1. Computational Infrastructure for Operations Research (COIN-OR), 2011. Solver de código aberto para a resolução de problemas de otimização de grande porte. Disponível em: $<$ http://www.coin-or.org/projects/Ipopt.xml>. Acesso em: 16 dez. 2011.

JIN, S.; RYAN, S. M. Capacity expansion in the integrated supply network for an electricity market. IEEE Transactions on Power Systems, v. 26, n. 4, p. 2275-2284, Nov. 2011.

LEYFFER, S.; LÓPEZ-CALVA, G.; NOCEDAL, J. Interior methods for mathematical programs with complementarity constraints. SIAM Journal on Optimization, Philadelphia, v. 17, n. 1, p. 52-77, Jan. 2006.

LIN, S.-Y.; HO, Y.-C.; LIN, C.-H. An ordinal optimization theory-based algorithm for solving the optimal power flow problem with discrete control variables. IEEE Transactions on Power Systems, v. 19, n. 1, p. 276-286, Feb. 2004.

LIU, M.; TSO, S. K.; CHENG, Y. An extended nonlinear primal-dual interior-point algorithm for reactive-power optimization of large-scale power systems with discrete control variables. IEEE Transactions on Power Systems, v. 17, n. 4, p. 982-991, Nov. 2002. 
LIU, W.-H. E.; PAPALEXOPOULOS, A. D.; TINNEY, W. F. Discrete shunt controls in a Newton optimal power flow. IEEE Transactions on Power Systems, v. 7, n. 4, p. 1509 -1518 , Nov. 1992.

MANKIW, N. G. Introdução à economia. Tradução Allan Vidigal Hastings, Elisete Paes e Lima; revisão técnica Carlos Roberto Martins Passos, Manuel José Nunes Pinto. 5. ed. São Paulo: Cengage Learning, 2009. 838 p.

MOMOH, J. A.; EL-HAWARY, M. E.; ADAPA, R. A review of selected optimal power flow literature to 1993: Part I: nonlinear and quadratic programming approaches. IEEE Transactions on Power Systems, v. 14, n. 1, p. 96-104, Feb. 1999.

. A review of selected optimal power flow literature to 1993: Part II: Newton, linear programming and interior point methods. IEEE Transactions on Power Systems, v. 14, n. 1, p. 105-111, Feb. 1999.

MONTICELli, A. J. Fluxo de carga em redes de energia elétrica. São Paulo: Edgard Blücher, 1983. 164 p.

MUÑOZ, J. R. A. Analysis and application of optimization techniques to power system security and electricity markets. 2008. 166 p. Tese (Doctor of Philosophy in Electrical and Computer Engineering) - University of Waterloo, Waterloo, 2008.

ÖSTERMARK, R. A multipurpose parallel genetic hybrid algorithm for non-linear non-convex programming problems. European Journal of Operational Research, v. 152, n. 1, p. 195-214, 2004.

PETOUSSIS, S. G.; ZHANG, X. P.; GODFREY, K. R. Electricity market equilibrium analysis based on nonlinear interior point algorithm with complementarity constraints. IET Generation, Transmission and Distribution, v. 1, n. 4, p. 603-612, 2007.

POLYAK, R. Modified barrier functions (theory and methods). Mathematical Programming, v. 54, n. 1-3, p. 177-222, Feb. 1992. 
POWER Systems Test Case Archive. 1999. Disponibiliza sistemas-teste IEEE para validação de metodologias e algoritmos de análise computacional de sistemas de energia elétrica. Disponível em: <http://www.ee.washington.edu/research/pstca > Acesso em: 26 dez. 2011.

QUINTANA, V. H.; TORRES, G. L.; MEDINA-PALOMO, J. Interior-point methods and their applications to power systems: a classification of publications and software codes. IEEE Transactions on Power Systems, v. 15, n. 1, p. 170-176, Feb. 2000.

RAGHUNATHAN, A. U.; BIEGLER, L. T. An interior point method for mathematical programs with complementarity constraints (MPCCs). SIAM Journal on Optimization, Philadelphia, v. 15, n. 3, p. 720-750, Mar. 2005.

RAMOS, J. L. M.; EXPÓSiTO, A. G.; CEREZO, J. C.; RUIZ, E. M.; SALINAS, F. C. A hybrid tool to assist the operator in reactive power/voltage control and optimization. IEEE Transactions on Power Systems, v. 10, n. 2, p. 760-768, May 1995.

RASHED, A. M. H.; KELLY, D. H. Optimal load flow solution using Lagrangian multipliers and the Hessian matrix. IEEE Transactions on Power Apparatus and Systems, PAS-93, n. 5, p. 1292-1297, Sept. 1974.

RIDER, M. J.; CASTRO, C. A.; BEDRIÑNANA, M. F.; GARCIA, A. V. Towards a fast and robust interior point method for power system applications. IEE Proceedings - Generation, Transmission and Distribution, v. 151, n. 5, p. 575-581, Sept. 2004.

ROSEHART, W. Optimal power flows incorporating network stability. In: IEEE POWER ENGINEERING SOCIETY WINTER MEETING, 2002, New York. Proceedings... New York: IEEE, 2002. v. 2.

ROSEHART, W.; ROMAN, C.; SCHELlENBERG, A. Optimal power flow with complementarity constraints. IEEE Transactions on Power Systems, v. 20, n. 2, p. 813-822, May 2005. 
SANTOS JR., A.; DECKMANN, S.; SOARES, S. A dual augmented Lagrangian approach for optimal power flow. IEEE Transactions on Power Systems, v. 3, n. 3, p. 1020-1025, Aug. 1988.

SASSON, A. M. Combined use of the Powell and Fletcher-Powell nonlinear programming methods for optimal load flows. IEEE Transactions on Power Apparatus and Systems, PAS-88, n. 10, p. 1530-1537, Oct. 1969.

SASSON, A. M.; VILORIA, F.; ABOYTES, F. Optimal load flow solution using the Hessian matrix. IEEE Transactions on Power Apparatus and Systems, PAS-92, n. 1, p. 31-41, Jan. 1973.

SOLER, E. M. Resolução do problema de fluxo de potência ótimo com variáveis de controle discretas. 2011. 108 f. Tese (Doutorado em Ciências, Programa de Engenharia Elétrica) — Escola de Engenharia de São Carlos, Universidade de São Paulo, São Carlos, 2011.

SOlER, E. M.; SOUSA, V. A. de; COSTA, G. R. M. da. A modified primal-dual logarithmic-barrier method for solving the optimal power flow problem with discrete and continuous control variables. European Journal of Operational Research, v. 222, n. 3, p. 616-622, 2012.

SOUSA, V. A. de. Resolução do problema de fluxo de potência ótimo reativo via método da função Lagrangiana barreira modificada. 2006. 141 f. Tese (Doutorado em Engenharia Elétrica) - Escola de Engenharia de São Carlos, Universidade de São Paulo, São Carlos, 2006.

SOUSA, V. A. de; BAPTISTA, E. C.; COSTA, G. R. M. da. Modified barrier method for optimal power flow problem. In: IEEE POWER ENGINEERING SOCIETY GENERAL MEETING, 2004, Denver. Proceedings... Denver: IEEE, 2004.

- Loss minimization by a predictor-corrector modified approach. Electric Power Systems Research, v. 79, n. 5, p. 803-808, May 2009. 
SOUZA, A. M. de. Estudos de casos em sistemas de energia elétrica por meio do fluxo de potência ótimo e da análise de sensibilidade. 2005. 137 f. Tese (Doutorado em Engenharia Elétrica) - Escola de Engenharia de São Carlos, Universidade de São Paulo, São Carlos, 2005.

STOTT, B. Decoupled newton load flow. IEEE Transactions on Power Apparatus and Systems, PAS-91, n. 5, p. 1955-1959, Sept. 1972.

STOTT, B.; ALSAÇ, O. Fast decoupled load flow. IEEE Transactions on Power Apparatus and Systems, PAS-93, n. 3, p. 859-869, May 1974.

SUN, D. I.; ASHLEY, B.; BREWER, B.; HUGHES, A.; TINNEY, W. F. Optimal power flow by Newton approach. IEEE Transactions on Power Apparatus and Systems, PAS-103, n. 10, p. 2864-2880, Oct. 1984.

TINNEY, W. F.; HART, C. E. Power flow solution by Newton's method. IEEE Transactions on Power Apparatus and Systems, PAS-86, n. 11, p. 1449-1460, Nov. 1967.

TODOROV, E. Implicit nonlinear complementarity: a new approach to contact dynamics. In: IEEE INTERNATIONAL CONFERENCE ON ROBOTICS AND AUTOMATION, 2010, Anchorage. Proceedings... Anchorage: IEEE, 2010.

TORRES, G. L.; QUINTANA, V. H. An interior point method for nonlinear optimal power flow using voltage rectangular coordinates. IEEE Transactions on Power Systems, v. 13, n. 4, p. 1211-1218, Nov. 1998.

WÄCHTER, A.; BIEGLER, L. T. On the implementation of a primal-dual interior point filter line search algorithm for large-scale nonlinear programming. Mathematical Programming, v. 106, n. 1, p. 25-57, 2006.

WU, Y.-C.; DEBS, A. S.; MARSTEN, R. E. A direct nonlinear predictor-corrector primal-dual interior point algorithm for optimal power flows. IEEE Transactions on Power Systems, v. 9, n. 2, p. 876-883, May 1994. 


\section{Apêndice A}

\section{Expressões Gerais dos Fluxos de Potência Ativa e Reativa}

Este apêndice traz a dedução das expressões gerais dos fluxos de potência ativa e reativa em um SEE. Para isso, as equações das correntes fasoriais em um ramo genérico $k$ - $m$ são determinadas através da análise dos modelos de linhas de transmissão e transformadores (em-fase e defasadores) pelas leis de Kirchhoff; matematicamente, essas equações são definidas em função dos parâmetros do ramo e das tensões fasoriais nas suas barras terminais. Com essas equações determinadas, as expressões gerais dos fluxos de potência ativa e reativa e, por conseguinte, das injeções de potência nas barras do sistema podem ser, enfim, obtidas. ${ }^{1}$

\section{A.1 Modelagem de Componentes do Sistema Elétrico}

Os componentes de um sistema de transmissão de energia elétrica podem ser divididos em dois grupos distintos. O primeiro deles é formado pelos componentes que estão ligados entre um nó qualquer da rede elétrica e o nó terra, como é o caso de geradores, cargas, e bancos de capacitores e reatores shunt; o segundo é formado pelos

\footnotetext{
${ }^{1}$ Em todas as formulações apresentadas neste apêndice, os parâmetros e as grandezas do sistema são expressos em valores por unidade (p.u.).
} 
componentes que estão ligados entre dois nós quaisquer do sistema, como é o caso de linhas de transmissão e transformadores em-fase e defasadores.

Neste trabalho, os geradores e as cargas são considerados como pertencentes à parte externa do sistema, e são modelados como injeções de potência ativa e reativa nas barras às quais estão conectados. A parte interna do sistema é constituída pelos demais componentes, ou seja, linhas de transmissão, transformadores, bancos de capacitores e reatores shunt etc. Os modelos desses componentes da parte interna de um SEE são apresentados a seguir.

\section{A.1.1 Linhas de Transmissão (MONTICELli, 1983)}

O modelo equivalente $\pi$ de uma linha de transmissão $k-m$, representado na Figura A.1, é definido por três parâmetros: a resistência série $r_{k m}$, a reatância série $x_{k m}$, e susceptância shunt $b_{k m}^{s h}$. A impedância do elemento série $z_{k m}$ é dada por:

$$
z_{k m}=r_{k m}+j x_{k m}
$$

A admitância série $y_{k m}$ é, portanto, dada por:

$$
\begin{aligned}
y_{k m} & =z_{k m}^{-1} \\
& =\frac{r_{k m}}{r_{k m}^{2}+x_{k m}^{2}}-j \frac{x_{k m}}{r_{k m}^{2}+x_{k m}^{2}} \\
& =g_{k m}+j b_{k m}
\end{aligned}
$$

ou seja, a condutância série $g_{k m}$ e a susceptância série $b_{k m}$ são dadas por:

$$
g_{k m}=\frac{r_{k m}}{r_{k m}^{2}+x_{k m}^{2}} \quad \text { e } \quad b_{k m}=-\frac{x_{k m}}{r_{k m}^{2}+x_{k m}^{2}}
$$

Como os parâmetros $r_{k m}$ e $x_{k m}$ são positivos, $g_{k m}$ é positivo e $b_{k m}$ é negativo (tipo indutivo). Já o parâmetro $b_{k m}^{s h}$ é positivo, pois o shunt de linha é do tipo capacitivo. 


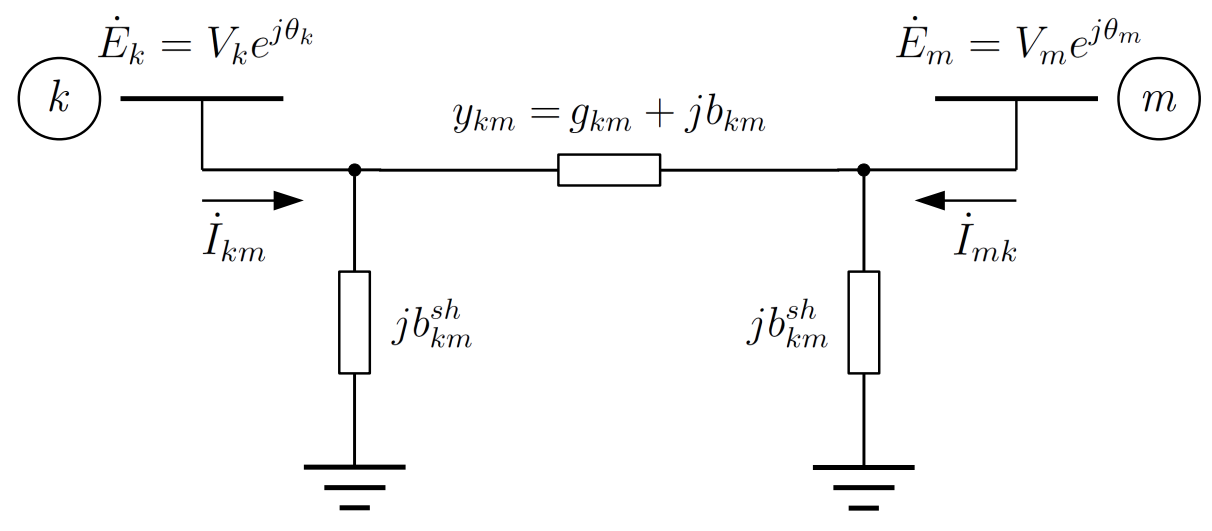

Figura A.1 - Modelo equivalente $\pi$ de uma linha de transmissão.

De acordo com a lei dos nós de Kirchhoff, a corrente $\dot{I}_{k m}$ (Figura A.1) é formada por uma componente série $\left(\dot{I}_{k m}^{z}\right)$ e uma componente shunt $\left(\dot{I}_{k m}^{s h}\right)$. Estas, por sua vez, podem ser expressas em função dos parâmetros da linha de transmissão e das tensões fasoriais $\dot{E}_{k}$ e $\dot{E}_{m}$ da seguinte forma:

$$
\begin{aligned}
\dot{I}_{k m} & =\dot{I}_{k m}^{z}+\dot{I}_{k m}^{s h} \\
& =y_{k m}\left(\dot{E}_{k}-\dot{E}_{m}\right)+j b_{k m}^{s h} \dot{E}_{k} \\
& =\left(y_{k m}+j b_{k m}^{s h}\right) \dot{E}_{k}-y_{k m} \dot{E}_{m}
\end{aligned}
$$

onde o ponto em $\dot{I}_{k m}, \dot{E}_{k}$ e $\dot{E}_{m}$ em (A.2) é usado para representar grandezas fasoriais, e:

$$
\dot{E}_{k}=V_{k} e^{j \theta_{k}} \quad \text { e } \quad \dot{E}_{m}=V_{m} e^{j \theta_{m}}
$$

Analogamente, a corrente $\dot{I}_{m k}$ (Figura A.1) é dada por:

$$
\begin{aligned}
\dot{I}_{m k} & =\dot{I}_{m k}^{z}+\dot{I}_{m k}^{s h} \\
& =y_{k m}\left(\dot{E}_{m}-\dot{E}_{k}\right)+j b_{k m}^{s h} \dot{E}_{m} \\
& =\left(y_{k m}+j b_{k m}^{s h}\right) \dot{E}_{m}-y_{k m} \dot{E}_{k}
\end{aligned}
$$




\section{A.1.2 Transformadores Em-Fase e Defasadores (GROUP, 1973)}

A representação de transformadores (em-fase e defasadores), dada na Figura A.2, consiste basicamente em um auto-transformador ideal com relação de transformação $a_{k m}$ : 1 e uma admitância série $y_{k m}$ (GROUP, 1973; CEPEL, 2004). Para transformadores em-fase, $a_{k m}$ é um número real $\left(a_{k m}=t_{k m}\right)$ e, para transformadores defasadores puros, $a_{k m}$ é um número complexo $\left(a_{k m}=e^{j \varphi_{k m}}\right)$.

No entanto, é importante ressaltar que, na operação de SEEs, os taps dos transformadores em-fase $\left(t_{k m}\right)$ e os ângulos de defasamento dos transformadores defasadores $\left(\varphi_{k m}\right)$ só podem ser ajustados por passos discretos, o que caracteriza a necessidade de uma modelagem discreta para essas variáveis de controle. Uma outra observação importante se refere à nomenclatura específica das barras terminais desses dispositivos de controle de tensão. No modelo da Figura A.2, a barra $k$ está mais "próxima" do tap e a barra $m$ está mais "próxima" da impedância. Por esse motivo, é comum dizer que a barra $k$ é a barra do tap e que a barra $m$ é a barra da impedância do ramo $k$ - $m$.

Feitas essas observações, considere o modelo genérico de um transformador (em-fase ou defasador) representado na Figura A.2, para o qual $a_{k m}=t_{k m} e^{j \varphi_{k m}}$. A relação entre as tensões nos nós $k$ e $p$ do transformador ideal é dada por:

$$
\begin{aligned}
\frac{\dot{E}_{p}}{\dot{E}_{k}} & =\frac{1}{a_{k m}} \\
& =\frac{1}{t_{k m} e^{j \varphi_{k m}}} \\
& =\frac{1}{t_{k m}} e^{-j \varphi_{k m}}
\end{aligned}
$$

o que implica em:

$$
\dot{E}_{p}=\frac{1}{t_{k m}} \dot{E}_{k} e^{-j \varphi_{k m}}
$$




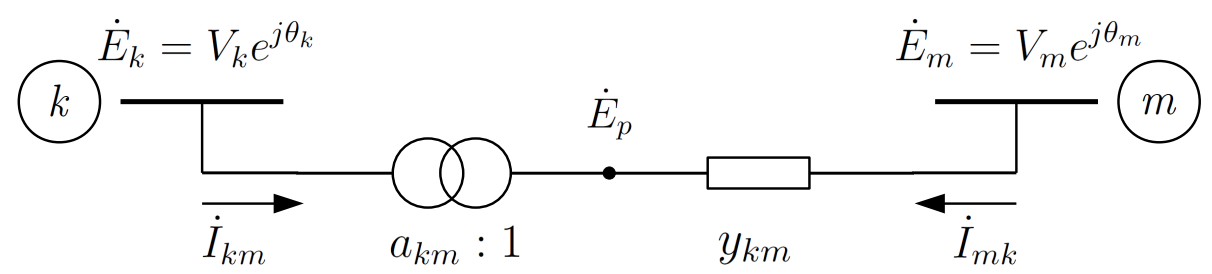

Figura A.2 - Modelo genérico de um transformador.

O fato de o transformador $k-p$ ser ideal implica também que as potências complexas na entrada e na saída são iguais, ou seja, não há dissipação de potência ativa ou reativa entre os nós $k$ e $p$ :

$$
\dot{E}_{k} \dot{I}_{k m}^{*}+\dot{E}_{p} \dot{I}_{m k}^{*}=0
$$

onde o asterisco em $\dot{I}_{k m}^{*}$ e $\dot{I}_{m k}^{*}$ representa o conjugado complexo dessas grandezas fasoriais.

Substituindo (A.5) em (A.6), obtém-se:

$$
\begin{aligned}
& \dot{E}_{k} \dot{I}_{k m}^{*}+\left(\frac{1}{t_{k m}} \dot{E}_{k} e^{-j \varphi_{k m}}\right) \dot{I}_{m k}^{*}=0 \\
& \dot{I}_{k m}^{*}=-\frac{1}{t_{k m}} e^{-j \varphi_{k m}} \dot{I}_{m k}^{*} \\
& \frac{\dot{I}_{k m}^{*}}{\dot{I}_{m k}^{*}}=-\frac{1}{t_{k m}} e^{-j \varphi_{k m}} \\
& \frac{\dot{I}_{k m}}{\dot{I}_{m k}}=-\frac{1}{t_{k m}} e^{j \varphi_{k m}}
\end{aligned}
$$

A corrente $\dot{I}_{m k}$ (Figura A.2) é formada por somente uma componente série, e pode ser expressa em função da admitância série $y_{k m}$ e das tensões fasoriais $\dot{E}_{p}$ e $\dot{E}_{m}$ da seguinte forma:

$$
\begin{aligned}
\dot{I}_{m k} & =\dot{I}_{m k}^{z} \\
& =y_{k m}\left(\dot{E}_{m}-\dot{E}_{p}\right)
\end{aligned}
$$


Substituindo (A.5) em (A.8):

$$
\begin{aligned}
\dot{I}_{m k} & =y_{k m}\left(\dot{E}_{m}-\frac{1}{t_{k m}} \dot{E}_{k} e^{-j \varphi_{k m}}\right) \\
& =y_{k m} \dot{E}_{m}+\left(-\frac{1}{t_{k m}} e^{-j \varphi_{k m}} y_{k m}\right) \dot{E}_{k}
\end{aligned}
$$

Já a corrente $\dot{I}_{k m}$ (Figura A.2) é determinada a partir de (A.7), ou seja:

$$
\dot{I}_{k m}=-\frac{1}{t_{k m}} e^{j \varphi_{k m}} \dot{I}_{m k}
$$

Substituindo (A.9) em (A.10), obtém-se:

$$
\begin{aligned}
\dot{I}_{k m} & =-\frac{1}{t_{k m}} e^{j \varphi_{k m}}\left[y_{k m} \dot{E}_{m}+\left(-\frac{1}{t_{k m}} e^{-j \varphi_{k m}} y_{k m}\right) \dot{E}_{k}\right] \\
& =\left(\frac{1}{t_{k m}^{2}} y_{k m}\right) \dot{E}_{k}+\left(-\frac{1}{t_{k m}} e^{j \varphi_{k m}} y_{k m}\right) \dot{E}_{m}
\end{aligned}
$$

\section{A.1.3 Bancos de Capacitores e Reatores Shunt}

Capacitores e reatores shunt são muito importantes para a operação de SEEs. Essencialmente, os capacitores e reatores shunt são uma forma de, respectivamente, injetar e absorver potência reativa no ponto onde são instalados. Eles podem ficar permanentemente ligados ou, quando atuam como dispositivos de controle de tensão, podem ser ligados e desligados conforme as necessidades do sistema.

Esses componentes, também conhecidos como shunts de barra, são modelados como susceptâncias diretamente conectadas às barras, como pode ser visto na Figura A.3. Se a susceptância do shunt for positiva, o shunt é do tipo capacitivo; caso ela seja negativa, o shunt é do tipo indutivo.

Quando o shunt de barra for formado por um conjunto de capacitores associados em paralelo, ele funciona como um importante dispositivo de controle de tensão nos SEEs. Estes são os bancos de capacitores. 


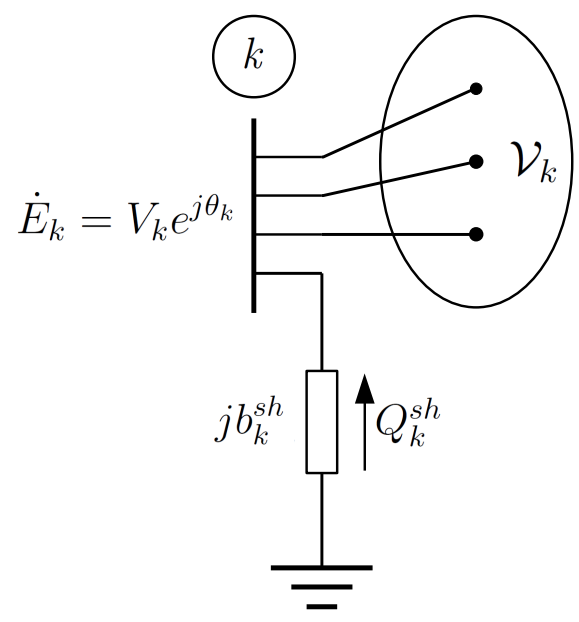

Figura A.3 - Modelo de um shunt de barra.

A atuação dos shunts de barra é feita por chaveamentos, e isso implica que a variável de controle associada a esses dispositivos, a susceptância equivalente do shunt da barra $\left(b_{k}^{s h}\right)$, seja modelada como uma variável de controle discreta ${ }^{2}$. Neste trabalho, considera-se que a variável controlada é a magnitude de tensão da própria barra $k$ através da injeção de potência reativa pelo shunt, cuja expressão é dada por:

$$
Q_{k}^{s h}\left(b_{k}^{s h}, V_{k}\right)=b_{k}^{s h} V_{k}^{2}
$$

\section{A.2 Expressões dos Fluxos de Potência}

A potência complexa $S_{k m}$ que deixa a barra $k$ pelo ramo $k-m$, cujas componentes ativa e reativa são representadas por $P_{k m}$ e $Q_{k m}$, é expressa em função da tensão fasorial na barra $k$ e do complexo conjugado da corrente fasorial $\dot{I}_{k m}$ da seguinte forma:

$$
S_{k m}=P_{k m}+j Q_{k m}=\dot{E}_{k} \dot{I}_{k m}^{*}
$$

\footnotetext{
${ }^{2}$ Neste trabalho, considera-se que os valores da susceptância equivalente $b_{k}^{s h}$ para os bancos de capacitores são determinados por todas combinações simples entre as susceptâncias de cada capacitor do banco de capacitores.
} 
Como a equação de $\dot{I}_{k m}^{*}$ não é conhecida, faz-se:

$$
S_{k m}^{*}=P_{k m}-j Q_{k m}=\dot{E}_{k}^{*} \dot{I}_{k m}
$$

Analogamente, a potência complexa $S_{m k}$ que deixa a barra $m$ pelo ramo $k$ - $m$ pode ser expressa por:

$$
S_{m k}^{*}=P_{m k}-j Q_{m k}=\dot{E}_{m}^{*} \dot{I}_{m k}
$$

Dessa forma, as expressões dos fluxos de potência ativa $P_{k m}$ e $P_{m k}$, e potência reativa $Q_{k m}$ e $Q_{m k}$ podem ser deduzidas, conforme será mostrado a seguir, a partir das equações das correntes $\dot{I}_{k m}$ e $\dot{I}_{m k}$ apresentadas na seção anterior.

\section{A.2.1 Linhas de Transmissão}

De acordo com (A.2) e (A.14), a potência complexa que flui da barra $k$ para a barra $m$ por uma linha de transmissão pode ser expressa por:

$$
S_{k m}^{*}=\dot{E}_{k}^{*}\left[\left(y_{k m}+j b_{k m}^{s h}\right) \dot{E}_{k}-y_{k m} \dot{E}_{m}\right]
$$

Substituindo as tensões fasoriais $\dot{E}_{k}$ e $\dot{E}_{m}$ em (A.16) por sua representação na forma polar (A.3):

$$
\begin{aligned}
S_{k m}^{*} & =V_{k} e^{-j \theta_{k}}\left[\left(y_{k m}+j b_{k m}^{s h}\right) V_{k} e^{j \theta_{k}}-y_{k m} V_{m} e^{j \theta_{m}}\right] \\
& =\left(y_{k m}+j b_{k m}^{s h}\right) V_{k}^{2}-y_{k m} V_{k} V_{m} e^{j\left(\theta_{m}-\theta_{k}\right)} \\
& =\left(y_{k m}+j b_{k m}^{s h}\right) V_{k}^{2}-y_{k m} V_{k} V_{m} e^{-j\left(\theta_{k}-\theta_{m}\right)}
\end{aligned}
$$


e adotando a notação $\theta_{k m}$ para a abertura angular entre os fasores das tensões nas barras $k$ e $m$, obtém-se:

$$
S_{k m}^{*}=\left(y_{k m}+j b_{k m}^{s h}\right) V_{k}^{2}-y_{k m} V_{k} V_{m} e^{-j \theta_{k m}}
$$

$$
\begin{aligned}
\operatorname{Como} e^{j \alpha} & =\cos (\alpha)+j \operatorname{sen}(\alpha) \text { : } \\
S_{k m}^{*} & =\left(y_{k m}+j b_{k m}^{s h}\right) V_{k}^{2}-y_{k m} V_{k} V_{m}\left[\cos \left(-\theta_{k m}\right)+j \operatorname{sen}\left(-\theta_{k m}\right)\right]
\end{aligned}
$$

e $\cos (-\alpha)=\cos (\alpha)$ e $\operatorname{sen}(-\alpha)=-\operatorname{sen}(\alpha)$, chega-se a:

$$
S_{k m}^{*}=\left(y_{k m}+j b_{k m}^{s h}\right) V_{k}^{2}-y_{k m} V_{k} V_{m}\left[\cos \left(\theta_{k m}\right)-j \operatorname{sen}\left(\theta_{k m}\right)\right]
$$

Finalmente, substituindo a admitância série $y_{k m}$ em (A.17) pela sua representação na forma retangular (A.1):

$$
S_{k m}^{*}=\left(g_{k m}+j b_{k m}+j b_{k m}^{s h}\right) V_{k}^{2}-\left(g_{k m}+j b_{k m}\right) V_{k} V_{m}\left[\cos \left(\theta_{k m}\right)-j \operatorname{sen}\left(\theta_{k m}\right)\right]
$$

chega-se à seguinte expressão para o fluxo de potência complexa em uma linha de transmissão:

$$
\begin{aligned}
S_{k m}^{*}= & {\left[g_{k m}+j\left(b_{k m}+b_{k m}^{s h}\right)\right] V_{k}^{2}-} \\
& V_{k} V_{m}\left[g_{k m} \cos \left(\theta_{k m}\right)+b_{k m} \operatorname{sen}\left(\theta_{k m}\right)\right]- \\
& j V_{k} V_{m}\left[b_{k m} \cos \left(\theta_{k m}\right)-g_{k m} \operatorname{sen}\left(\theta_{k m}\right)\right]
\end{aligned}
$$

Lembrando que as parcelas imaginárias de (A.18) correspondem a $-j Q_{k m}$, as expressões para os fluxos $P_{k m}$ e $Q_{k m}$ são obtidas identificando-se as partes reais e 
imaginárias dessa equação complexa:

$$
\begin{aligned}
& P_{k m}=g_{k m} V_{k}^{2}-V_{k} V_{m}\left[g_{k m} \cos \left(\theta_{k m}\right)+b_{k m} \operatorname{sen}\left(\theta_{k m}\right)\right] \\
& Q_{k m}=-\left(b_{k m}+b_{k m}^{s h}\right) V_{k}^{2}+V_{k} V_{m}\left[b_{k m} \cos \left(\theta_{k m}\right)-g_{k m} \operatorname{sen}\left(\theta_{k m}\right)\right]
\end{aligned}
$$

Os fluxos $P_{m k}$ e $Q_{m k}$, obtidos analogamente, são expressos por:

$$
\begin{aligned}
& P_{m k}=g_{k m} V_{m}^{2}-V_{k} V_{m}\left[g_{k m} \cos \left(\theta_{k m}\right)-b_{k m} \operatorname{sen}\left(\theta_{k m}\right)\right] \\
& Q_{m k}=-\left(b_{k m}+b_{k m}^{s h}\right) V_{m}^{2}+V_{k} V_{m}\left[b_{k m} \cos \left(\theta_{k m}\right)+g_{k m} \operatorname{sen}\left(\theta_{k m}\right)\right]
\end{aligned}
$$

As perdas de potência ativa em uma linha de transmissão são determinadas pela diferença entre a potência ativa que deixa a barra $k$ e a potência que chega à barra $m$. Matematicamente, essas perdas podem ser expressas como:

$$
\begin{aligned}
P_{k m}^{\text {perdas }}= & P_{k m}+P_{m k} \\
= & \left\{g_{k m} V_{k}^{2}-V_{k} V_{m}\left[g_{k m} \cos \left(\theta_{k m}\right)+b_{k m} \operatorname{sen}\left(\theta_{k m}\right)\right]\right\}+ \\
& \left\{g_{k m} V_{m}^{2}-V_{k} V_{m}\left[g_{k m} \cos \left(\theta_{k m}\right)-b_{k m} \operatorname{sen}\left(\theta_{k m}\right)\right]\right\} \\
= & g_{k m}\left[V_{k}^{2}+V_{m}^{2}-2 V_{k} V_{m} \cos \left(\theta_{k m}\right)\right]
\end{aligned}
$$

\section{A.2.2 Transformadores Em-Fase e Defasadores}

De acordo com (A.11) e (A.14), a potência complexa que flui da barra $k$ para a barra $m$ por um transformador pode ser expressa por:

$$
S_{k m}^{*}=\dot{E}_{k}^{*}\left[\left(\frac{1}{t_{k m}^{2}} y_{k m}\right) \dot{E}_{k}+\left(-\frac{1}{t_{k m}} e^{j \varphi_{k m}} y_{k m}\right) \dot{E}_{m}\right]
$$


Substituindo as tensões fasoriais $\dot{E}_{k}$ e $\dot{E}_{m}$ em (A.24) por sua representação na forma polar (A.3):

$$
\begin{aligned}
S_{k m}^{*} & =V_{k} e^{-j \theta_{k}}\left[\left(\frac{1}{t_{k m}^{2}} y_{k m}\right) V_{k} e^{j \theta_{k}}+\left(-\frac{1}{t_{k m}} e^{j \varphi_{k m}} y_{k m}\right) V_{m} e^{j \theta_{m}}\right] \\
& =\left(\frac{1}{t_{k m}^{2}} y_{k m}\right) V_{k}^{2}+\left(-\frac{1}{t_{k m}} y_{k m}\right) V_{k} V_{m} e^{j\left(\theta_{m}-\theta_{k}+\varphi_{k m}\right)} \\
& =\left(\frac{1}{t_{k m}^{2}} y_{k m}\right) V_{k}^{2}+\left(-\frac{1}{t_{k m}} y_{k m}\right) V_{k} V_{m} e^{-j\left(\theta_{k}-\theta_{m}-\varphi_{k m}\right)}
\end{aligned}
$$

e adotando a notação $\theta_{k m}$ para a abertura angular entre os fasores das tensões nas barras $k$ e $m$, obtém-se:

$$
\begin{gathered}
S_{k m}^{*}=\left(\frac{1}{t_{k m}^{2}} y_{k m}\right) V_{k}^{2}+\left(-\frac{1}{t_{k m}} y_{k m}\right) V_{k} V_{m} e^{-j\left(\theta_{k m}-\varphi_{k m}\right)} \\
\text { Como } e^{j \alpha}=\cos (\alpha)+j \operatorname{sen}(\alpha): \\
S_{k m}^{*}=\left(\frac{1}{t_{k m}^{2}} y_{k m}\right) V_{k}^{2}+\left(-\frac{1}{t_{k m}} y_{k m}\right) V_{k} V_{m}\left[\cos \left(-\left(\theta_{k m}-\varphi_{k m}\right)\right)+j \operatorname{sen}\left(-\left(\theta_{k m}-\varphi_{k m}\right)\right)\right] \\
\text { e } \cos (-\alpha)=\cos (\alpha) \text { e } \operatorname{sen}(-\alpha)=-\operatorname{sen}(\alpha), \operatorname{chega}-\operatorname{se~a:~} \\
S_{k m}^{*}=\left(\frac{1}{t_{k m}^{2}} y_{k m}\right) V_{k}^{2}+\left(-\frac{1}{t_{k m}} y_{k m}\right) V_{k} V_{m}\left[\cos \left(\theta_{k m}-\varphi_{k m}\right)-j \operatorname{sen}\left(\theta_{k m}-\varphi_{k m}\right)\right] \quad(\mathrm{A} .25)
\end{gathered}
$$

Finalmente, substituindo a admitância série $y_{k m}$ em (A.25) pela sua representação na forma retangular:

$$
S_{k m}^{*}=\left(g_{k m}+j b_{k m}\right) \frac{1}{t_{k m}^{2}} V_{k}^{2}-\left(g_{k m}+j b_{k m}\right) \frac{1}{t_{k m}} V_{k} V_{m}\left[\cos \left(\theta_{k m}-\varphi_{k m}\right)-j \operatorname{sen}\left(\theta_{k m}-\varphi_{k m}\right)\right]
$$


chega-se à seguinte expressão para o fluxo de potência complexa em um transformador:

$$
\begin{aligned}
S_{k m}^{*}= & \left(g_{k m}+j b_{k m}\right) \frac{1}{t_{k m}^{2}} V_{k}^{2}- \\
& \frac{1}{t_{k m}} V_{k} V_{m}\left[g_{k m} \cos \left(\theta_{k m}-\varphi_{k m}\right)+b_{k m} \operatorname{sen}\left(\theta_{k m}-\varphi_{k m}\right)\right]- \\
& j \frac{1}{t_{k m}} V_{k} V_{m}\left[b_{k m} \cos \left(\theta_{k m}-\varphi_{k m}\right)-g_{k m} \operatorname{sen}\left(\theta_{k m}-\varphi_{k m}\right)\right]
\end{aligned}
$$

Lembrando que as parcelas imaginárias de (A.26) correspondem a $-j Q_{k m}$, as expressões para os fluxos $P_{k m}$ e $Q_{k m}$ são obtidas identificando-se as partes reais e imaginárias dessa equação complexa:

$$
\begin{aligned}
& P_{k m}=g_{k m} \frac{1}{t_{k m}^{2}} V_{k}^{2}-\frac{1}{t_{k m}} V_{k} V_{m}\left[g_{k m} \cos \left(\theta_{k m}-\varphi_{k m}\right)+b_{k m} \operatorname{sen}\left(\theta_{k m}-\varphi_{k m}\right)\right] \\
& Q_{k m}=-b_{k m} \frac{1}{t_{k m}^{2}} V_{k}^{2}+\frac{1}{t_{k m}} V_{k} V_{m}\left[b_{k m} \cos \left(\theta_{k m}-\varphi_{k m}\right)-g_{k m} \operatorname{sen}\left(\theta_{k m}-\varphi_{k m}\right)\right]
\end{aligned}
$$

Os fluxos $P_{m k}$ e $Q_{m k}$, obtidos analogamente, são expressos por:

$$
\begin{aligned}
& P_{m k}=g_{k m} V_{m}^{2}-\frac{1}{t_{k m}} V_{k} V_{m}\left[g_{k m} \cos \left(\theta_{k m}-\varphi_{k m}\right)-b_{k m} \operatorname{sen}\left(\theta_{k m}-\varphi_{k m}\right)\right] \\
& Q_{m k}=-b_{k m} V_{m}^{2}+\frac{1}{t_{k m}} V_{k} V_{m}\left[b_{k m} \cos \left(\theta_{k m}-\varphi_{k m}\right)+g_{k m} \operatorname{sen}\left(\theta_{k m}-\varphi_{k m}\right)\right]
\end{aligned}
$$

Assim como para linhas de transmissão, as perdas de potência ativa em um transformador são determinadas pela diferença entre a potência ativa que deixa a barra $k$ e a potência que chega à barra $m$. Matematicamente, essas perdas podem ser expressas 
como:

$$
\begin{aligned}
P_{k m}^{\text {perdas }}= & P_{k m}+P_{m k} \\
= & \left\{g_{k m} \frac{1}{t_{k m}^{2}} V_{k}^{2}-\frac{1}{t_{k m}} V_{k} V_{m}\left[g_{k m} \cos \left(\theta_{k m}-\varphi_{k m}\right)+b_{k m} \operatorname{sen}\left(\theta_{k m}-\varphi_{k m}\right)\right]\right\} \\
& \left\{g_{k m} V_{m}^{2}-\frac{1}{t_{k m}} V_{k} V_{m}\left[g_{k m} \cos \left(\theta_{k m}-\varphi_{k m}\right)-b_{k m} \operatorname{sen}\left(\theta_{k m}-\varphi_{k m}\right)\right]\right\} \\
= & g_{k m}\left[\frac{1}{t_{k m}^{2}} V_{k}^{2}+V_{m}^{2}-2 \frac{1}{t_{k m}} V_{k} V_{m} \cos \left(\theta_{k m}-\varphi_{k m}\right)\right]
\end{aligned}
$$

\section{A.2.3 Expressões Gerais dos Fluxos}

Comparando-se as expressões dos fluxos de potência ativa e reativa em linhas de transmissão e transformadores, elas podem ser agrupadas nas seguintes expressões gerais:

$$
\begin{aligned}
P_{k m}= & g_{k m} \frac{1}{t_{k m}^{2}} V_{k}^{2}-\frac{1}{t_{k m}} V_{k} V_{m}\left[g_{k m} \cos \left(\theta_{k m}-\varphi_{k m}\right)+b_{k m} \operatorname{sen}\left(\theta_{k m}-\varphi_{k m}\right)\right] \\
Q_{k m}= & -\left(b_{k m} \frac{1}{t_{k m}^{2}}+b_{k m}^{s h}\right) V_{k}^{2}+ \\
& \frac{1}{t_{k m}} V_{k} V_{m}\left[b_{k m} \cos \left(\theta_{k m}-\varphi_{k m}\right)-g_{k m} \operatorname{sen}\left(\theta_{k m}-\varphi_{k m}\right)\right] \\
P_{m k}= & g_{k m} V_{m}^{2}-\frac{1}{t_{k m}} V_{k} V_{m}\left[g_{k m} \cos \left(\theta_{k m}-\varphi_{k m}\right)-b_{k m} \operatorname{sen}\left(\theta_{k m}-\varphi_{k m}\right)\right] \\
Q_{m k}= & -\left(b_{k m}+b_{k m}^{s h}\right) V_{m}^{2}+ \\
& \frac{1}{t_{k m}} V_{k} V_{m}\left[b_{k m} \cos \left(\theta_{k m}-\varphi_{k m}\right)+g_{k m} \operatorname{sen}\left(\theta_{k m}-\varphi_{k m}\right)\right]
\end{aligned}
$$

Para linhas de transmissão, $t_{k m}=1$ e $\varphi_{k m}=0$; para transformadores em-fase, $b_{k m}^{s h}=0$ e $\varphi_{k m}=0$; para defasadores, $b_{k m}^{s h}=0$ e $t_{k m}=1$.

As equações (A.32), (A.33), (A.34) e (A.35) foram desenvolvidas para a barra $k$ sendo a barra do tap e a barra $m$ sendo a barra da impedância de ramos transformadores. 
No entanto, a barra $k$ pode ser, simultaneamente, tanto a barra do tap de um ramo quanto a barra da impedância de outro. Portanto, deve-se restringir o uso das expressões (A.32) e (A.33) aos casos em que a barra $k$ é a barra do tap do ramo.

As expressões gerais dos fluxos $P_{k m}$ e $Q_{k m}$ para os casos em que a barra $k$ é a barra da impedância são obtidas, respectivamente, pela troca do índice $k$ pelo índice $m$ (e vice-versa) das variáveis associadas às tensões fasoriais nas equações (A.34) e (A.35). Essa troca resulta nas seguintes expressões gerais:

$$
\begin{aligned}
& P_{k m}=g_{k m} V_{k}^{2}-\frac{1}{t_{k m}} V_{k} V_{m}\left[g_{k m} \cos \left(\theta_{k m}+\varphi_{k m}\right)+b_{k m} \operatorname{sen}\left(\theta_{k m}+\varphi_{k m}\right)\right] \\
& Q_{k m}=-b_{k m} V_{k}^{2}+\frac{1}{t_{k m}} V_{k} V_{m}\left[b_{k m} \cos \left(\theta_{k m}+\varphi_{k m}\right)-g_{k m} \operatorname{sen}\left(\theta_{k m}+\varphi_{k m}\right)\right]
\end{aligned}
$$

onde o parâmetro $b_{k m}^{s h}$ foi omitido de (A.37) por esta referir-se somente ao cálculo do fluxo de potência reativa em transformadores (em-fase ou defasadores), para os quais $b_{k m}^{s h}=0$.

\section{A.3 Expressões das Injeções de Potência}

A formulação das expressões de injeção de potência ativa e reativa nas barras do sistema é baseada na lei dos nós de Kirchhoff, segundo a qual as injeções $P_{k}$ e $Q_{k}$ são, respectivamente, iguais à soma dos fluxos de potência ativa e reativa que deixam a barra $k$.

A imposição da conservação das potências ativa e reativa em cada barra do sistema elétrico através da lei dos nós Kirchhoff pode ser matematicamente expressa por:

$$
\begin{aligned}
& P_{k}=\sum_{m \in \mathcal{V}_{k}} P_{k m}\left(V_{k}, V_{m}, \theta_{k}, \theta_{m}, t_{k m}, \varphi_{k m}\right) \\
& Q_{k}+Q_{k}^{s h}\left(V_{k}, b_{k}^{s h}\right)=\sum_{m \in \mathcal{V}_{k}} Q_{k m}\left(V_{k}, V_{m}, \theta_{k}, \theta_{m}, t_{k m}, \varphi_{k m}\right)
\end{aligned}
$$

onde $P_{k m}$ e $Q_{k m}$ são determinados por (A.32), (A.33), (A.36) e (A.37), dependendo se o ramo $k-m$ for uma linha de transmissão ou transformador. 


\title{
Apêndice B
}

\section{Relatórios dos Fluxos Ótimos}

\section{B.1 Sistema IEEE 14 Barras}

\author{
CONVERGÊNCIA EM 6 ITERAÇÕES
}

\begin{tabular}{|c|c|c|c|c|c|c|c|c|c|c|c|}
\hline \multirow[b]{2}{*}{ BARRA } & \multirow[b]{2}{*}{$\mathrm{TP}$} & \multirow{2}{*}{\multicolumn{2}{|c|}{ NOME }} & \multicolumn{5}{|c|}{ ESTADO DO SISTEMA } & \multicolumn{3}{|c|}{ FLUXOS NOS RAMOS } \\
\hline & & & & & TENSÃO & ANG. & MW & MVAR & BARRA & MW & MVAR \\
\hline 1 & SL & ‘Bus & 1 & HV & 1.1000 & 0.00 & 231.3 & -24.5 & $\begin{array}{l}2 \\
5\end{array}$ & $\begin{array}{r}155.84 \\
75.49\end{array}$ & $\begin{array}{r}-24.94 \\
0.44\end{array}$ \\
\hline GER & REAT & 'Bus & 41.8 & [LIMI & TES : MÁX. & -4.63 & MÍN.$=$ & -40]$. & $\begin{array}{l}1 \\
3 \\
4 \\
5\end{array}$ & $\begin{array}{r}-151.86 \\
73.13 \\
55.81 \\
41.22\end{array}$ & $\begin{array}{r}33.92 \\
0.65 \\
-3.94 \\
-1.49\end{array}$ \\
\hline GER & $\mathrm{CR}$ & 'Bus & 30.4 & [LIMI & TES: MÁX. & $\begin{array}{l}-11.78 \\
.=40\end{array}$ & -94.2 & $\begin{array}{l}11.4 \\
0 .]\end{array}$ & $\begin{array}{l}2 \\
4\end{array}$ & $\begin{array}{l}-71.01 \\
-23.19\end{array}$ & $\begin{array}{l}5.76 \\
5.63\end{array}$ \\
\hline 4 & CG & 'Bus & 4 & HV & 1.0676 & -9.57 & -47.8 & 3.9 & $\begin{array}{l}2 \\
3\end{array}$ & $\begin{array}{r}-54.28 \\
23.53\end{array}$ & $\begin{array}{r}6.61 \\
-5.48\end{array}$ \\
\hline
\end{tabular}




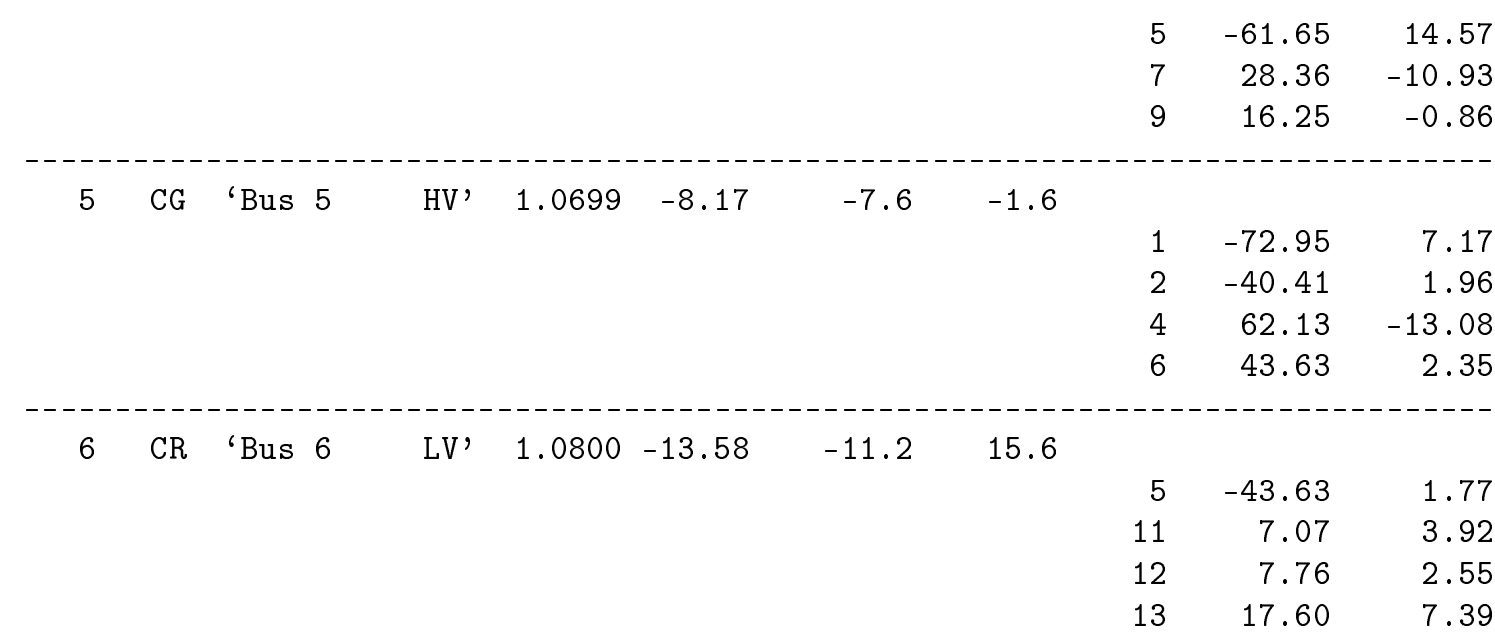

GER.REAT. $=23.1$ [LIMITES: MÁX. $=24$. MÍN. $=-6$.

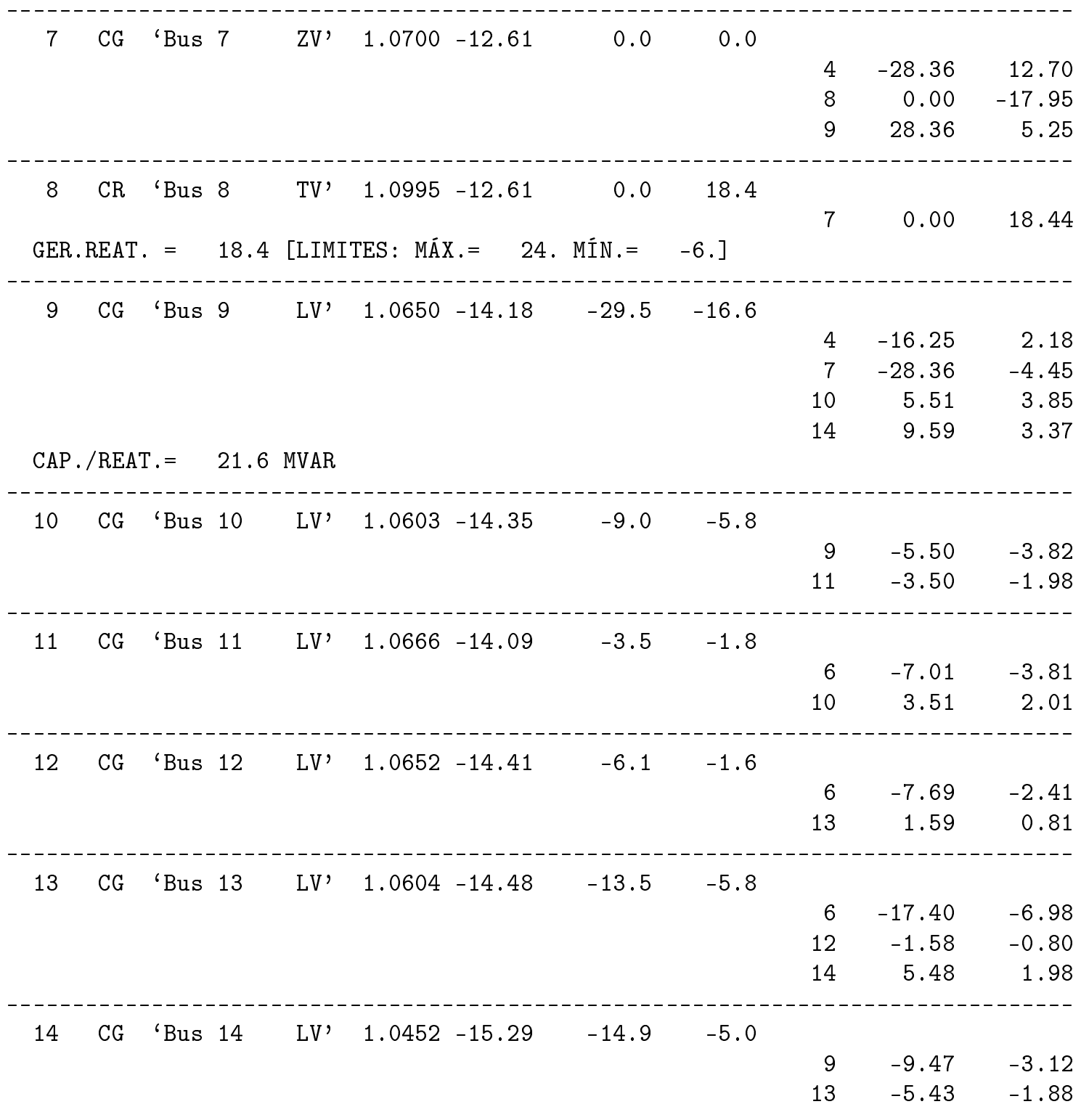


TAPS DOS TRANSFORMADORES

$\begin{array}{ccccccc}\text { BARRA } & \text { BARRA } & \text { BARRA } & \text { TAP } & \text { TAP } & \text { TAP } & \text { PASSO } \\ \text { INICIAL } & \text { FINAL } & \text { CRTL. } & & \text { MİN. } & \text { MÁX. } & \text { DISCRETO } \\ -1 & 7 & 7 & 1.0200 & 0.880 & 1.120 & 0.0100 \\ 4 & 9 & 9 & 1.0100 & 0.880 & 1.120 & 0.0100 \\ 4 & 6 & 6 & 0.9900 & 0.880 & 1.120 & 0.0100\end{array}$

SUSCEPTÂNCIA EQUIVALENTE DOS BANCOS DE CAPACITORES

\begin{tabular}{ccc} 
BARRA BSHUNT VALORES DISCRETOS \\
\hdashline$\quad 0.190 \quad 1$ & $\rightarrow>0.000$ \\
& $2 \rightarrow-0.050$ \\
3 & $\rightarrow>0.150$ \\
4 & $\rightarrow>0.190$ \\
5 & $\rightarrow>0.200$ \\
6 & $\rightarrow>0.240$ \\
7 & $\rightarrow>0.340$ \\
8 & $\rightarrow 0.390$
\end{tabular}

PARÂMETROS DO ALGORITMO DE DISCRETIZAÇÃO DAS VARIÁVEIS

$\begin{array}{rl}\text { TAP: } \quad \begin{array}{l}\text { gama } \\ \text { tau }\end{array}=10 & 10-05 \\ \text { BSHUNT }: & \text { gama }=1 \mathrm{e}-05 \\ \text { tau }=10 \\ \text { beta }=1 \\ \text { tol }=0.0001\end{array}$




\title{
B.2 Sistema IEEE 30 Barras
}

\author{
CONVERGÊNCIA EM 6 ITERAÇÕES
}

PERDAS $=16.5273 \mathrm{MW}$

ESTADO DO SISTEMA

FLUXOS NOS RAMOS

\begin{tabular}{|c|c|c|c|c|c|c|c|c|c|c|}
\hline BARRA & TP & \multicolumn{2}{|l|}{ NOME } & TENSÃO & ANG. & MW & MVAR & BARRA & MW & MVAR \\
\hline \multirow[t]{3}{*}{1} & SL & 'Glen Lyn & 132 & 1.1000 & 0.00 & 259.9 & 6.8 & & & \\
\hline & & & & & & & & 2 & 172.71 & -6.21 \\
\hline & & & & & & & & 3 & 87.22 & 13.06 \\
\hline \multirow[t]{5}{*}{2} & $\mathrm{CR}$ & 'Claytor & 132 & 1.0761 & -4.86 & 18.3 & 37.3 & & & \\
\hline & & & & & & & & 1 & -167.97 & 17.27 \\
\hline & & & & & & & & 4 & 43.60 & 8.94 \\
\hline & & & & & & & & 5 & 82.34 & 5.25 \\
\hline & & & & & & & & 6 & 60.33 & 5.84 \\
\hline \multicolumn{2}{|c|}{ GER . REAT . } & $=50.0$ & [LIMI & TES: MÁX & $=50$. & MÍN . = & -40]$. & & & \\
\hline \multirow[t]{3}{*}{3} & CG & 'Kumis & 132 & 1.0502 & -6.84 & -2.4 & -1.2 & & & \\
\hline & & & & & & & & 1 & -84.30 & -4.75 \\
\hline & & & & & & & & 4 & 81.90 & 3.55 \\
\hline \multirow[t]{5}{*}{4} & CG & 'Hancock & 132 & 1.0389 & -8.45 & -7.6 & -1.6 & & & \\
\hline & & & & & & & & 2 & -42.61 & -8.00 \\
\hline & & & & & & & & 3 & -81.10 & -1.70 \\
\hline & & & & & & & & 6 & 72.03 & -13.96 \\
\hline & & & & & & & & 12 & 44.08 & 22.06 \\
\hline \multirow[t]{3}{*}{5} & $\mathrm{CR}$ & 'Fieldale & 132 & 1.0388 & -13.10 & -94.2 & 17.9 & & & \\
\hline & & & & & & & & 2 & -79.56 & 4.09 \\
\hline & & & & & & & & 7 & -14.64 & 13.80 \\
\hline \multicolumn{2}{|c|}{ GER . REAT } & 36.9 & [LIMI & TES: MÁX & $=40$. & MÍN. $=$ & -40]$. & & & \\
\hline \multirow[t]{8}{*}{6} & CG & 'Roanoke & 132 & 1.0366 & -10.12 & 0.0 & 0.0 & & & \\
\hline & & & & & & & & 2 & -58.48 & -2.31 \\
\hline & & & & & & & & 4 & -71.43 & 15.54 \\
\hline & & & & & & & & 7 & 37.98 & -3.34 \\
\hline & & & & & & & & 8 & 29.54 & -8.90 \\
\hline & & & & & & & & 9 & 27.91 & -4.58 \\
\hline & & & & & & & & 10 & 16.00 & 2.05 \\
\hline & & & & & & & & 28 & 18.49 & 1.53 \\
\hline \multirow[t]{3}{*}{7} & CG & 'Blaine & 132 & 1.0295 & -11.83 & -22.8 & -10.9 & & & \\
\hline & & & & & & & & 5 & 14.82 & -14.44 \\
\hline & & & & & & & & 6 & -37.62 & 3.54 \\
\hline
\end{tabular}




\begin{tabular}{|c|c|c|c|c|c|c|c|c|c|c|}
\hline \multirow{3}{*}{8} & $\mathrm{CR}$ & 'Reusens & 132 & 1.0368 & -10.84 & -30.0 & 10.0 & \multirow[b]{2}{*}{6} & \multirow[b]{2}{*}{-29.44} & \multirow[b]{2}{*}{8.78} \\
\hline & & & & & & & & & & \\
\hline & & & & & & & & 28 & -0.56 & 1.22 \\
\hline \multicolumn{2}{|c|}{ GER . REAT } & 40.0 & [LIMI & TES : MÁX & $\mathrm{X} .=$ & MÍN. = & -10]$. & & & \\
\hline \multirow[t]{4}{*}{9} & CG & 'Roanoke & 1.0 & 1.0682 & -13.07 & 0.0 & 0.0 & & & \\
\hline & & & & & & & & 6 & -27.91 & 6.06 \\
\hline & & & & & & & & 11 & 0.00 & -13.14 \\
\hline & & & & & & & & 10 & 27.91 & 7.08 \\
\hline \multirow{8}{*}{10} & --- & -------- & --- & ------- & -------- & ------ & ------ & -- & -------- & ------- \\
\hline & $\mathrm{CG}$ & 'Roanoke & 33 & 1.0613 & -14.62 & -5.8 & -2.0 & & & \\
\hline & & & & & & & & 6 & -16.00 & -0.78 \\
\hline & & & & & & & & 9 & -27.91 & -6.28 \\
\hline & & & & & & & & 20 & 9.06 & 4.08 \\
\hline & & & & & & & & 17 & 5.36 & 4.79 \\
\hline & & & & & & & & 21 & 15.95 & 11.81 \\
\hline & & & & & & & & 22 & 7.74 & 5.77 \\
\hline \multicolumn{3}{|c|}{ CAP. $/$ REAT.$=$} & MVAR & & & & & & & \\
\hline \multirow{3}{*}{11} & 然 & 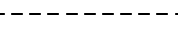 & ---1 & & 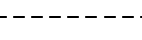 & & & & &.-- \\
\hline & $\mathrm{CR}$ & 'Roanoke & 11 & 1.0937 & -13.07 & 0.0 & 13.5 & & & \\
\hline & & & & & & & & 9 & 0.00 & 13.45 \\
\hline \multicolumn{2}{|c|}{ GER . REAT } & $=13.5$ & [LIMI & TES: MÁX & $X .=24$ & MÍN. = & -6]$. & & & \\
\hline \multirow[t]{6}{*}{12} & $\mathrm{CG}$ & 'Hancock & 33 & 1.0714 & -13.86 & -11.2 & -7.5 & & & \\
\hline & & & & & & & & 4 & -44.08 & -17.07 \\
\hline & & & & & & & & 13 & 0.00 & -3.27 \\
\hline & & & & & & & & 14 & 7.86 & 2.54 \\
\hline & & & & & & & & 15 & 17.81 & 7.33 \\
\hline & & & & & & & & 16 & 7.21 & 2.97 \\
\hline & $-\ldots$ & 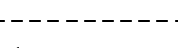 & 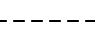 & & -------- & 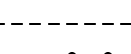 & ---- & -- & ----- & ----- \\
\hline \multirow[t]{2}{*}{13} & $\mathrm{CR}$ & 'Hancock & 11 & 1.0756 & -13.86 & 0.0 & 3.3 & & & \\
\hline & & & & & & & & 12 & 0.00 & 3.28 \\
\hline \multicolumn{2}{|c|}{ GER. REAT } & $=3.3$ & [LIMI & TES: MÁX & $x .=24$ & MÍN. = & -6]$. & & & \\
\hline \multirow{4}{*}{14} & --- & -------- & ---- & ------- & -------- & ------- & -------- & --- & -----1 & --- \\
\hline & CG & 'Bus 14 & 33, & 1.0564 & -14.72 & -6.2 & -1.6 & & & \\
\hline & & & & & & & & 12 & -7.79 & -2.39 \\
\hline & & & & & & & & 15 & 1.59 & 0.79 \\
\hline \multirow[t]{5}{*}{15} & CG & 'Bus 15 & 33, & 1.0516 & -14.79 & -8.2 & -2.5 & & & \\
\hline & & & & & & & & 12 & -17.59 & -6.91 \\
\hline & & & & & & & & 14 & -1.58 & -0.78 \\
\hline & & & & & & & & 18 & 5.98 & 1.22 \\
\hline & & & & & & & & 23 & 5.00 & 3.97 \\
\hline \multirow[t]{3}{*}{16} & $\mathrm{CG}$ & 'Bus 16 & 33 & 1.0595 & -14.44 & -3.5 & -1.8 & & & \\
\hline & & & & & & & & 12 & -7.16 & -2.87 \\
\hline & & & & & & & & 17 & 3.66 & 1.07 \\
\hline \multirow[t]{3}{*}{17} & CG & 'Bus 17 & 33 & 1.0558 & -14.77 & -9.0 & -5.8 & & & \\
\hline & & & & & & & & 16 & -3.65 & -1.04 \\
\hline & & & & & & & & 10 & -5.35 & -4.76 \\
\hline \multirow[t]{3}{*}{18} & CG & 'Bus 18 & $33^{\prime}$ & 1.0430 & -15.40 & -3.2 & -0.9 & & & \\
\hline & & & & & & & & 15 & -5.94 & -1.14 \\
\hline & & & & & & & & 19 & 2.74 & 0.24 \\
\hline
\end{tabular}




\begin{tabular}{|c|c|c|c|c|c|c|c|c|c|c|c|}
\hline 19 & CG & 'Bus & 19 & $33^{\prime}$ & 1.0410 & -15.58 & -9.5 & -3.4 & $\begin{array}{l}18 \\
20\end{array}$ & $\begin{array}{l}-2.74 \\
-6.76\end{array}$ & $\begin{array}{l}-0.23 \\
-3.17\end{array}$ \\
\hline 20 & CG & 'Bus & 20 & 33 , & 1.0453 & -15.40 & -2.2 & -0.7 & $\begin{array}{l}19 \\
10\end{array}$ & $\begin{array}{r}6.78 \\
-8.98\end{array}$ & $\begin{array}{r}3.20 \\
-3.90\end{array}$ \\
\hline 21 & CG & 'Bus & 21 & 33 , & 1.0477 & -15.02 & -17.5 & -11.2 & $\begin{array}{l}10 \\
22\end{array}$ & $\begin{array}{r}-15.83 \\
-1.67\end{array}$ & $\begin{array}{r}-11.54 \\
0.34\end{array}$ \\
\hline 22 & CG & 'Bus & 22 & 33 , & 1.0478 & -15.00 & 0.0 & 0.0 & $\begin{array}{l}10 \\
21 \\
24\end{array}$ & $\begin{array}{r}-7.68 \\
1.67 \\
6.01\end{array}$ & $\begin{array}{r}-5.65 \\
-0.34 \\
5.99\end{array}$ \\
\hline 23 & CG & 'Bus & 23 & 33 , & 1.0392 & -15.11 & -3.2 & -1.6 & $\begin{array}{l}15 \\
24\end{array}$ & $\begin{array}{r}-4.96 \\
1.76\end{array}$ & $\begin{array}{r}-3.90 \\
2.30\end{array}$ \\
\hline CAP & CG & 'Bus & 4.3 & MVAR & 1.0310 & -15.20 & -8.7 & -6.7 & $\begin{array}{l}22 \\
23 \\
25\end{array}$ & $\begin{array}{l}-5.93 \\
-1.75 \\
-1.02\end{array}$ & $\begin{array}{r}-5.88 \\
-2.28 \\
1.45\end{array}$ \\
\hline 25 & CG & 'Bus & 25 & 33 , & 1.0282 & -14.87 & 0.0 & 0.0 & $\begin{array}{l}24 \\
26 \\
27\end{array}$ & $\begin{array}{r}1.02 \\
3.54 \\
-4.57\end{array}$ & $\begin{array}{r}-1.44 \\
2.37 \\
-0.92\end{array}$ \\
\hline 26 & CG & 'Bus & 26 & 33 , & 1.0108 & -15.29 & -3.5 & -2.3 & 25 & -3.50 & -2.30 \\
\hline 27 & CG & 'Clov & verdle & 33 & 1.0350 & -14.42 & 0.0 & 0.0 & $\begin{array}{l}25 \\
28 \\
29 \\
30\end{array}$ & $\begin{array}{r}4.59 \\
-17.87 \\
6.19 \\
7.09\end{array}$ & $\begin{array}{r}0.96 \\
-4.28 \\
1.66 \\
1.66\end{array}$ \\
\hline 28 & CG & 'Clov & verdle & 132 & 1.0325 & -10.70 & 0.0 & 0.0 & $\begin{array}{r}27 \\
8 \\
6\end{array}$ & $\begin{array}{r}17.87 \\
0.57 \\
-18.43\end{array}$ & $\begin{array}{r}5.53 \\
-3.50 \\
-2.03\end{array}$ \\
\hline 29 & CG & 'Bus & 29 & 33 , & 1.0154 & -15.62 & -2.4 & -0.9 & $\begin{array}{l}27 \\
30\end{array}$ & $\begin{array}{r}-6.10 \\
3.70\end{array}$ & $\begin{array}{r}-1.50 \\
0.60\end{array}$ \\
\hline 30 & CG & 'Bus & 30 & 33 , & 1.0041 & -16.48 & -10.6 & -1.9 & $\begin{array}{l}27 \\
29\end{array}$ & $\begin{array}{l}-6.93 \\
-3.67\end{array}$ & $\begin{array}{l}-1.36 \\
-0.54\end{array}$ \\
\hline
\end{tabular}


TAPS DOS TRANSFORMADORES

\begin{tabular}{|c|c|c|c|c|c|c|}
\hline BARRA & BARRA & BARRA & TAP & TAP & TAP & PASSO \\
\hline INICIAL & FINAL & CRTL. & & MÍN. & MÁX. & DISCRETO \\
\hline & 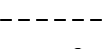 & & & $\operatorname{son}$ & 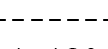 & 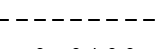 \\
\hline 6 & 9 & 9 & 0.9800 & 0.880 & 1.120 & 0.0100 \\
\hline- & -- & $5--x+3$ & & --- & ----- & -------- \\
\hline 6 & 10 & 10 & 0.9700 & 0.880 & 1.120 & 0.0100 \\
\hline & - & & & --- & ----- & ------- \\
\hline 4 & 12 & 12 & 0.9300 & 0.880 & 1.120 & 0.0100 \\
\hline & - & & & & & ------- \\
\hline 28 & 27 & 27 & 0.9800 & 0.880 & 1.120 & 0.0100 \\
\hline
\end{tabular}

SUSCEPTÂNCIA EQUIVALENTE DOS BANCOS DE CAPACITORES

\begin{tabular}{|c|c|c|c|}
\hline BARRA & BSHUNT & VALORES & DISCRETOS \\
\hline & --- & -----1 & ------- \\
\hline 10 & 0.190 & $1->$ & 0.000 \\
\hline & & $2->$ & 0.050 \\
\hline & & $3->$ & 0.150 \\
\hline & & $4->$ & $0.190 *$ \\
\hline & & $5->$ & 0.200 \\
\hline & & $6->$ & 0.240 \\
\hline & & $7->$ & 0.340 \\
\hline & & $8->$ & 0.390 \\
\hline & & ----- & ------- \\
\hline 24 & 0.000 & $1->$ & $0.000 *$ \\
\hline & & $2->$ & 0.040 \\
\hline & & $3->$ & 0.050 \\
\hline & & $4->$ & 0.090 \\
\hline
\end{tabular}

PARÂMETROS DO ALGORITMO DE DISCRETIZAÇÃO DAS VARIÁVEIS

$\begin{array}{rl}\text { TAP: } \quad \begin{array}{l}\text { gama } \\ \text { tau }\end{array}=10 & 10-05 \\ \text { BSHUNT: } & \text { gama }=1 \mathrm{e}-05 \\ \text { tau }=10 \\ \text { beta }=1 \\ \text { tol }=0.0001\end{array}$




\section{B.3 Sistema IEEE 57 Barras}

CONVERGÊNCIA EM 30 ITERAÇÕES

PERDAS $=18.5917 \mathrm{MW}$

ESTADO DO SISTEMA

FLUXOS NOS RAMOS

\begin{tabular}{|c|c|c|c|c|c|c|c|c|c|c|}
\hline BARRA & $\mathrm{TP}$ & NOME & & TENSÃO & ANG. & MW & MVAR & BARRA & MW & MVAR \\
\hline \multirow[t]{7}{*}{1} & $\mathrm{SL}$ & 'Kanawha & V1, & 1.1000 & 0.00 & 414.4 & 8.8 & & & \\
\hline & & & & & & & & 2 & 44.02 & -0.17 \\
\hline & & & & & & & & 5 & 35.58 & 0.39 \\
\hline & & & & & & & & 2 & 93.72 & 1.79 \\
\hline & & & & & & & & 15 & 106.10 & 6.48 \\
\hline & & & & & & & & 16 & 60.45 & -2.18 \\
\hline & & & & & & & & 17 & 74.52 & 2.49 \\
\hline \multirow[t]{7}{*}{2} & $\mathrm{CR}$ & 'Turner & V1, & 1.0917 & -1.23 & -3.0 & -38.0 & & & \\
\hline & & & & & & & & 1 & -43.71 & -2.05 \\
\hline & & & & & & & & 3 & 23.22 & -4.94 \\
\hline & & & & & & & & 4 & 29.86 & -4.16 \\
\hline & & & & & & & & 5 & 29.43 & -3.30 \\
\hline & & & & & & & & 1 & -93.12 & -7.50 \\
\hline & & & & & & & & 3 & 51.32 & -16.06 \\
\hline
\end{tabular}

GER.REAT. $=50.0$ [LIMITES: MÁX. $=50$. MÍN. $=-17$.

\begin{tabular}{|c|c|c|c|c|c|c|c|c|c|c|}
\hline 3 & CR & 'Logan & V1 & 1.0892 & -3.53 & -1.0 & 39.0 & & & \\
\hline & & & & & & & & 2 & -23.00 & 3.25 \\
\hline & & & & & & & & 4 & 5.15 & 2.16 \\
\hline & & & & & & & & 2 & -50.61 & 13.20 \\
\hline & & & & & & & & 4 & 23.70 & 12.91 \\
\hline & & & & & & & & 15 & 43.76 & 7.48 \\
\hline GEF & REAT & $=60.0$ & [LIM & TES: MÁX. & 60. & $\operatorname{MINN} .=$ & -10]$. & & & \\
\hline 4 & CG & 'Sprigg & V1, & 1.0821 & -3.87 & 0.0 & 0.0 & & & \\
\hline & & & & & & & & 2 & -29.42 & 3.49 \\
\hline & & & & & & & & 3 & -5.13 & -2.87 \\
\hline & & & & & & & & 5 & -3.47 & 3.70 \\
\hline & & & & & & & & 7 & 5.65 & 9.27 \\
\hline & & & & & & & & 9 & 7.50 & 8.13 \\
\hline & & & & & & & & 3 & -23.63 & -14.91 \\
\hline & & & & & & & & 5 & -0.89 & 0.50 \\
\hline & & & & & & & & 6 & 16.39 & -10.19 \\
\hline & & & & & & & & 18 & 14.56 & 3.60 \\
\hline & & & & & & & & 18 & 18.44 & -0.72 \\
\hline 5 & CG & 'Bus 5 & V1' & 1.0811 & -3.78 & -13.0 & -4.0 & & & \\
\hline & & & & & & & & 1 & -35.01 & -0.98 \\
\hline
\end{tabular}




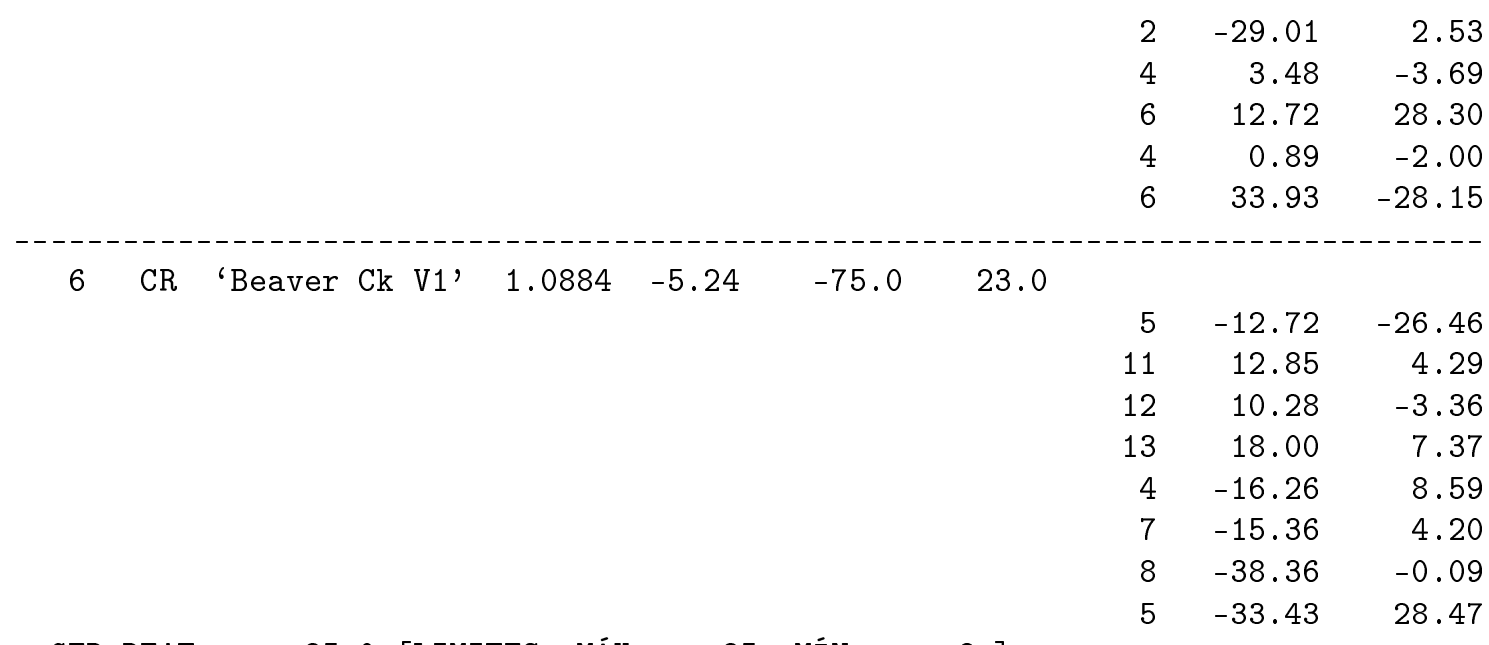

GER.REAT. $=25.0$ [LIMITES: MÁX. $=25$. MÍN. $=$-8.]

\begin{tabular}{|c|c|c|c|c|c|c|c|c|c|c|}
\hline \multirow[t]{7}{*}{7} & CG & 'Bus 7 & V1, & 1.0866 & -4.44 & 0.0 & 0.0 & & & \\
\hline & & & & & & & & 4 & -5.65 & -9.07 \\
\hline & & & & & & & & 8 & -28.31 & -7.65 \\
\hline & & & & & & & & 9 & 26.48 & 11.18 \\
\hline & & & & & & & & 6 & 15.40 & -5.60 \\
\hline & & & & & & & & 8 & -71.03 & -5.63 \\
\hline & & & & & & & & 29 & 63.10 & 16.77 \\
\hline \multirow[t]{5}{*}{8} & $\mathrm{CR}$ & 'Clinch Rv & V1 & 1.1000 & -2.05 & 300.0 & 40.8 & & & \\
\hline & & & & & & & & 7 & 28.31 & 8.93 \\
\hline & & & & & & & & 6 & 38.78 & -0.58 \\
\hline & & & & & & & & 9 & 161.29 & 24.94 \\
\hline & & & & & & & & 7 & 71.62 & 7.52 \\
\hline \multicolumn{2}{|c|}{ GER . REAT } & 62.8 & [LIMI & TES: MÁX & $=200$. & MÍN.$=$ & 140.] & & & \\
\hline \multirow[t]{11}{*}{9} & $\mathrm{CR}$ & 'Saltville & V1 & 1.0757 & -5.87 & -121.0 & -29.0 & & & \\
\hline & & & & & & & & 4 & -7.50 & -7.58 \\
\hline & & & & & & & & 7 & -26.48 & -10.41 \\
\hline & & & & & & & & 10 & 19.99 & -2.06 \\
\hline & & & & & & & & 14 & 2.31 & 2.54 \\
\hline & & & & & & & & 8 & -159.10 & -17.03 \\
\hline & & & & & & & & 10 & 10.55 & -0.84 \\
\hline & & & & & & & & 11 & 11.63 & 3.63 \\
\hline & & & & & & & & 12 & 4.93 & -6.70 \\
\hline & & & & & & & & 13 & 4.72 & 2.08 \\
\hline & & & & & & & & 55 & 17.95 & 7.38 \\
\hline GEP & REAT & -3.0 & [LIMI & TES: MÁX & 9. & MÍN.= & -3]$. & & & \\
\hline \multirow[t]{6}{*}{10} & CG & 'Bus 10 & v1, & 1.0715 & -6.74 & -5.0 & -2.0 & & & \\
\hline & & & & & & & & 9 & -19.88 & 2.36 \\
\hline & & & & & & & & 11 & -3.44 & 2.57 \\
\hline & & & & & & & & 9 & -10.51 & -1.53 \\
\hline & & & & & & & & 12 & -2.66 & -11.88 \\
\hline & & & & & & & & 51 & 31.49 & 6.48 \\
\hline \multirow[t]{3}{*}{11} & CG & 'Tazewell & V1, & 1.0696 & -6.30 & 0.0 & 0.0 & & & \\
\hline & & & & & & & & 6 & -12.70 & -3.98 \\
\hline & & & & & & & & 10 & 3.45 & -2.54 \\
\hline
\end{tabular}




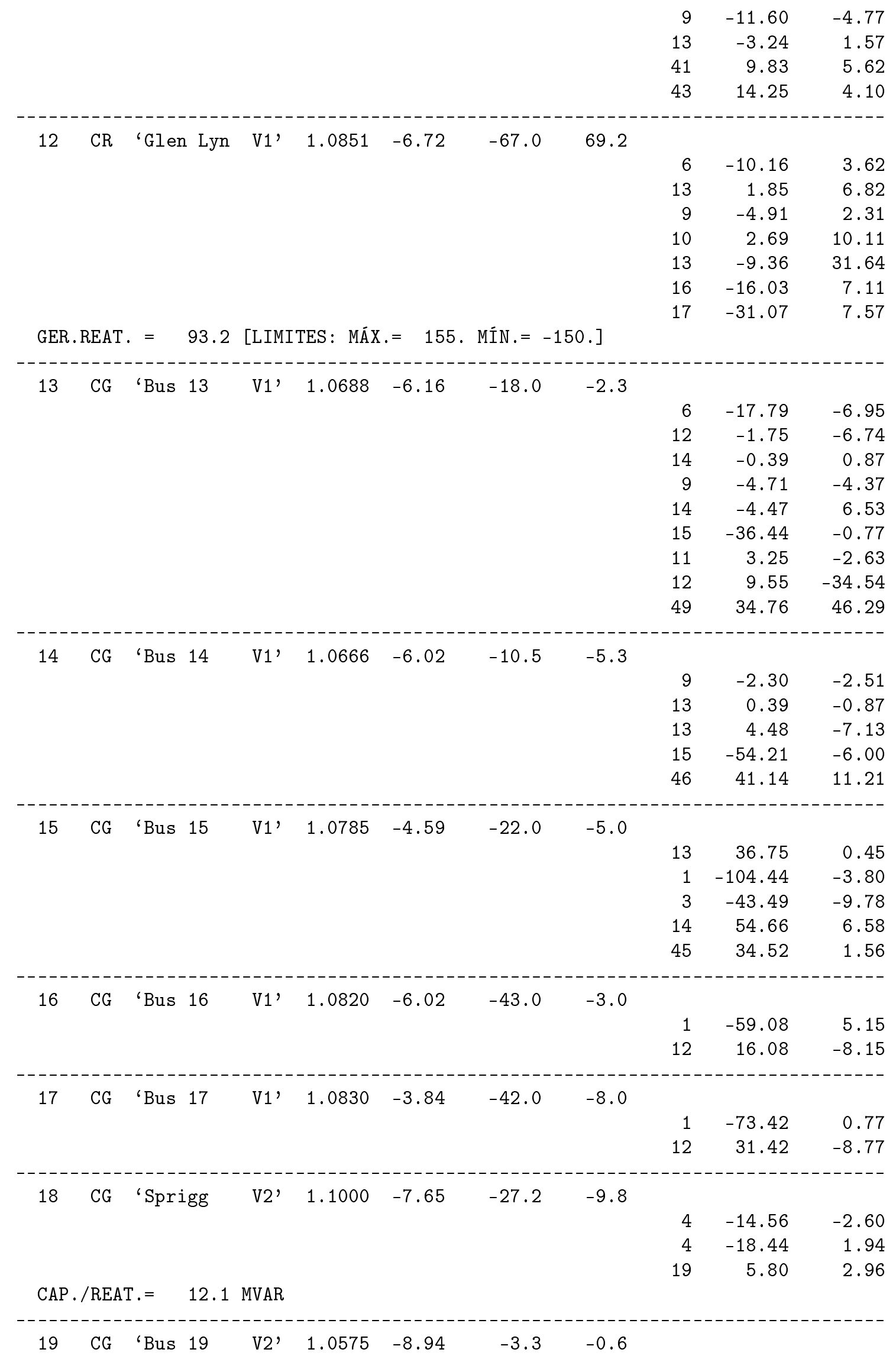




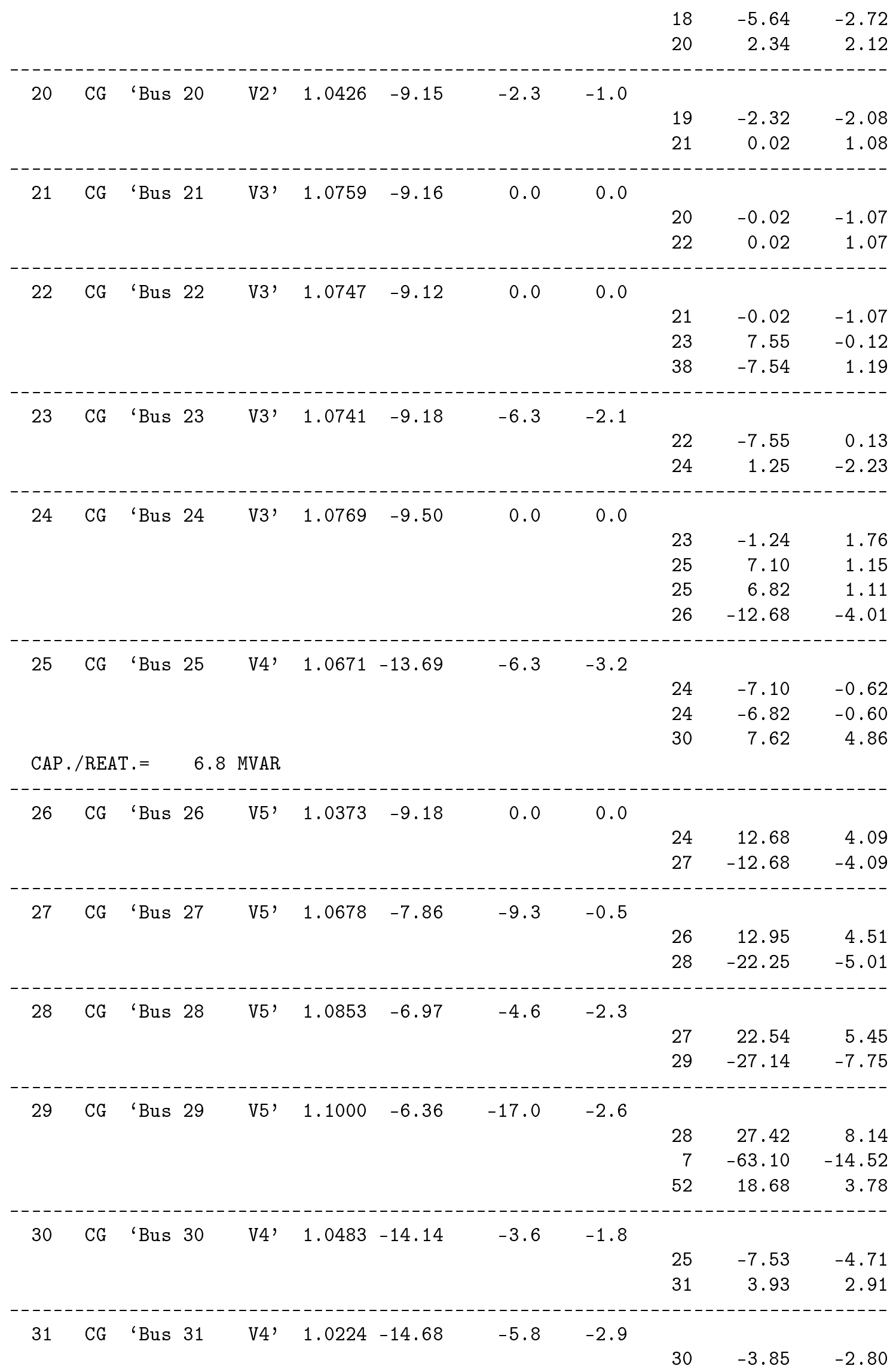




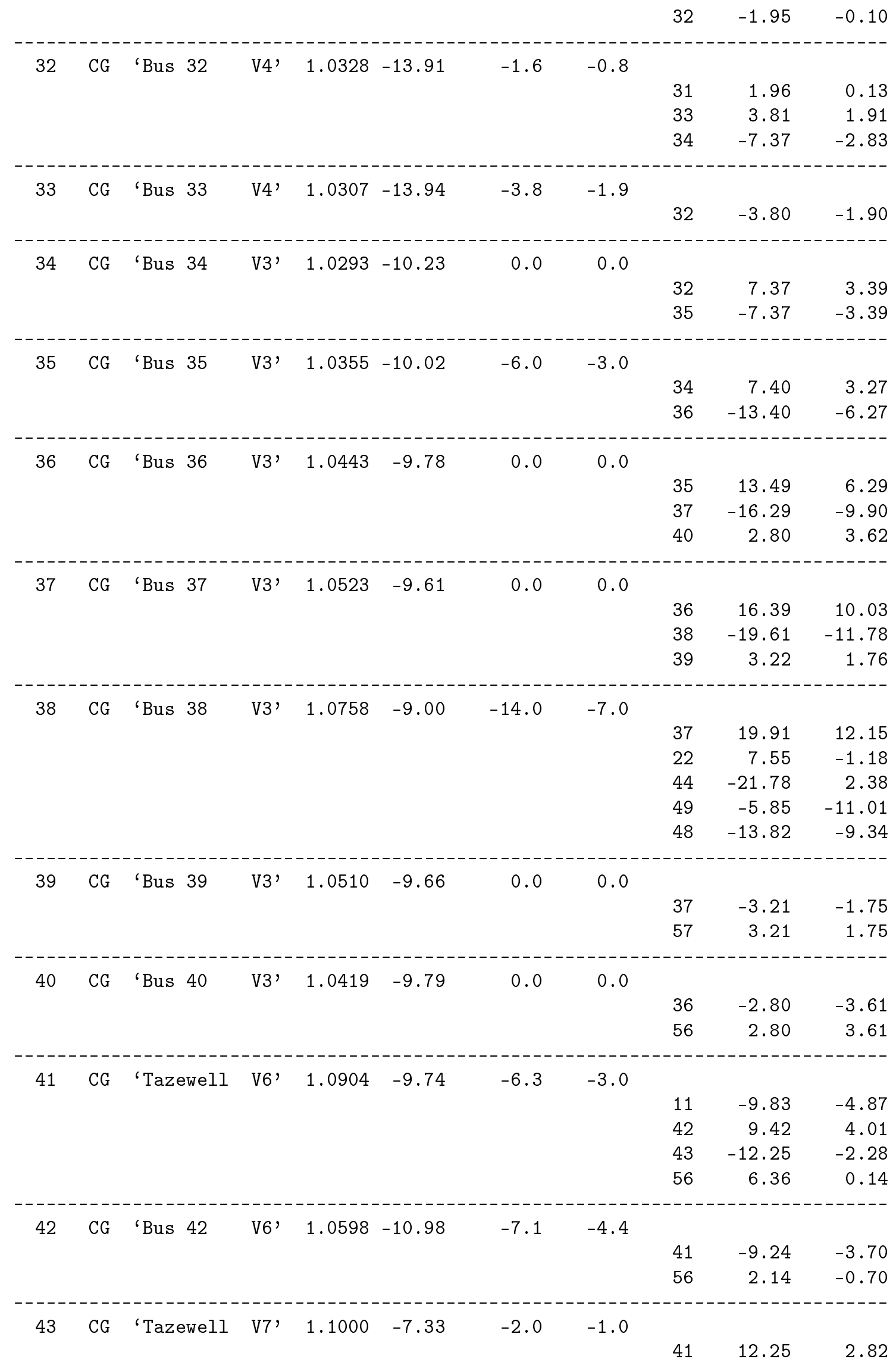




\begin{tabular}{|c|c|c|c|c|c|c|c|c|c|c|}
\hline & & & & & & & & 11 & -14.25 & -3.82 \\
\hline \multirow{4}{*}{44} & & & & & & & & & & \\
\hline & CG & 'Bus 44 & V3, & 1.0804 & -8.34 & -12.0 & -1.8 & & & \\
\hline & & & & & & & & 38 & 21.90 & -2.25 \\
\hline & & & & & & & & 45 & -33.90 & 0.45 \\
\hline \multirow[t]{3}{*}{45} & CG & 'Bus 45 & V3' & 1.1000 & -6.29 & 0.0 & 0.0 & & & \\
\hline & & & & & & & & 15 & -34.52 & -0.53 \\
\hline & & & & & & & & 44 & 34.52 & 0.53 \\
\hline & - & -------- & ---- & ------ & ------ & ----- & ----- & -1 & ----- & ----- \\
\hline \multirow[t]{3}{*}{46} & $\mathrm{CG}$ & 'Bus 46 & V3, & 1.1000 & -7.44 & 0.0 & 0.0 & & & \\
\hline & & & & & & & & 14 & -41.14 & -10.12 \\
\hline & & & & & & & & 47 & 41.14 & 10.12 \\
\hline \multirow{3}{*}{47} & CG & 'Bus 47 & V3, & 1.0853 & -8.67 & -29.7 & -11.6 & --1 & ----- & ------ \\
\hline & & & & & & & & 46 & -40.80 & -9.30 \\
\hline & & & & & & & & 48 & 11.10 & -2.30 \\
\hline \multirow{4}{*}{48} & CG & 'Bus 48 & V3, & 1.0840 & -8.82 & 0.0 & 0.0 & & --- & ------ \\
\hline & & & & & & & & 47 & -11.08 & 2.32 \\
\hline & & & & & & & & 49 & -2.82 & -11.77 \\
\hline & & & & & & & & 38 & 13.90 & 9.45 \\
\hline \multirow[t]{5}{*}{49} & CG & 'Bus 49 & V3, & 1.1000 & -9.11 & -18.0 & -8.5 & & & \\
\hline & & & & & & & & 48 & 2.93 & 11.65 \\
\hline & & & & & & & & 50 & 7.83 & 10.43 \\
\hline & & & & & & & & 13 & -34.76 & -41.65 \\
\hline & & & & & & & & 38 & 6.00 & 11.07 \\
\hline & --1 r y & ----1 & - & -----1 & --- & ---- & ---- & ---1 - & -------- & ------- \\
\hline \multirow[t]{3}{*}{50} & CG & 'Bus 50 & V3, & 1.0822 & -9.19 & -21.0 & -10.5 & & & \\
\hline & & & & & & & & 49 & -7.71 & -10.25 \\
\hline & & & & & & & & 51 & -13.29 & -0.25 \\
\hline--- & -1 & -------- & & ------- & ----- & ----- & ----- & --- & -------- & ----- \\
\hline \multirow[t]{3}{*}{51} & CG & 'Bus 51 & V3, & 1.1000 & -7.80 & -18.0 & -5.3 & & & \\
\hline & & & & & & & & 50 & 13.49 & 0.58 \\
\hline & & & & & & & & 10 & -31.49 & -5.88 \\
\hline \multirow[t]{3}{*}{52} & CG & 'Bus 52 & V5, & ----- & -7.79 & -4.9 & -2.2 & & ------- & \\
\hline & & & & & & & & 29 & -18.25 & -3.22 \\
\hline & & & & & & & & 53 & 13.35 & 1.02 \\
\hline 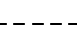 & -1 & ------- & --- & ----1 & ----- & ----- & ---- & & -------- & ---- \\
\hline \multirow[t]{3}{*}{53} & CG & 'Bus 53 & V5 & 1.0590 & -8.42 & -20.0 & -10.0 & & & \\
\hline & & & & & & & & 52 & -13.23 & -0.86 \\
\hline & & & & & & & & 54 & -6.77 & -1.85 \\
\hline \multicolumn{3}{|c|}{ CAP. $/$ REAT.$=$} & MVAR & & & & & & & \\
\hline \multirow{4}{*}{54} & & & & & & & & & & \\
\hline & CG & 'Bus 54 & V5' & 1.0752 & -7.80 & -4.1 & -1.4 & & & \\
\hline & & & & & & & & 53 & 6.85 & 1.95 \\
\hline & & & & & & & & 55 & -10.95 & -3.35 \\
\hline \multirow[t]{3}{*}{55} & CG & 'Saltville & V5 & 1.1000 & -6.88 & -6.8 & -3.4 & & & \\
\hline & & & & & & & & 54 & 11.15 & 3.61 \\
\hline & & & & & & & & 9 & -17.95 & -7.01 \\
\hline
\end{tabular}

56 CG 'Bus $56 \quad$ V6' $1.0579-11.44 \quad-7.6 \quad-2.2$ 


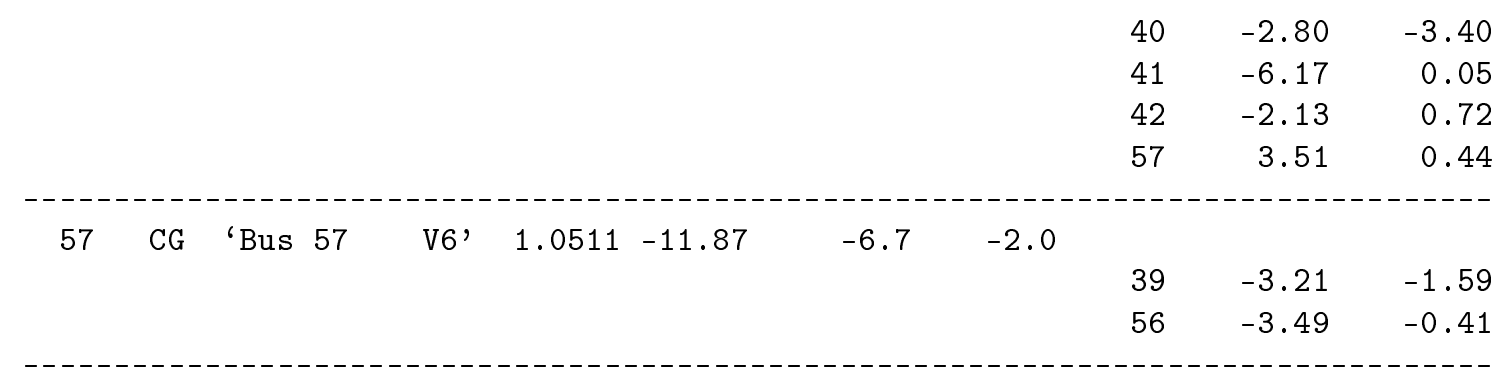

TAPS DOS TRANSFORMADORES

\begin{tabular}{|c|c|c|c|c|c|c|}
\hline $\begin{array}{c}\text { BARRA } \\
\text { INICIAL }\end{array}$ & $\begin{array}{l}\text { BARRA } \\
\text { FINAL }\end{array}$ & $\begin{array}{l}\text { BARRA } \\
\text { CRTL. }\end{array}$ & TAP & $\begin{array}{l}\text { TAP } \\
\text { MÍN. }\end{array}$ & $\begin{array}{l}\text { TAP } \\
\text { MÁX. }\end{array}$ & $\begin{array}{c}\text { PASSO } \\
\text { DISCRETO }\end{array}$ \\
\hline & & & & & & \\
\hline 4 & 7 & 7 & 0.9800 & 0.880 & 1.120 & 0.0100 \\
\hline & & & & & & \\
\hline 4 & 9 & 9 & 0.9700 & 0.880 & 1.120 & 0.0100 \\
\hline & & & & & & \\
\hline 5 & 6 & 6 & 0.9400 & 0.880 & 1.120 & 0.0100 \\
\hline & & & & & & \\
\hline 4 & 18 & 18 & 0.9700 & 0.880 & 1.120 & 0.0100 \\
\hline 4 & 18 & 18 & 0.9892 & 0.880 & 1.120 & 0.0100 \\
\hline & & 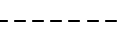 & (1) & ----- & ----- & -- \\
\hline 21 & 20 & 20 & 1.0400 & 0.880 & 1.120 & 0.0100 \\
\hline & -- & -- & ------ & ----- & ----- & -- \\
\hline 24 & 25 & 25 & 1.0000 & 0.880 & 1.120 & 0.0100 \\
\hline & & -- & ------ & ---- & ----- & ------- \\
\hline 24 & 25 & 25 & 1.0000 & 0.880 & 1.120 & 0.0100 \\
\hline & & -- & ------- & ----- & ------ & ---- \\
\hline 24 & 26 & 26 & 1.0400 & 0.880 & 1.120 & 0.0100 \\
\hline & & --- & ------ & ---- & ------ & ---- \\
\hline 7 & 29 & 29 & 0.9797 & 0.880 & 1.120 & 0.0100 \\
\hline--- & --- & --- & ------ & ------ & ------- & ------- \\
\hline 34 & 32 & 32 & 0.9700 & 0.880 & 1.120 & 0.0100 \\
\hline--1 - & --1 > & --- & -------- & ------- & ------- & ------- \\
\hline 11 & 41 & 41 & 0.9500 & 0.880 & 1.120 & 0.0100 \\
\hline.- & - & --- & -------- & ------ & ------- & -------- \\
\hline 15 & 45 & 45 & 0.9795 & 0.880 & 1.120 & 0.0100 \\
\hline- & 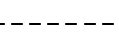 & $x_{0}+2$ & ------- & & ---- & --- \\
\hline 14 & 46 & 46 & 0.9609 & 0.880 & 1.120 & 0.0100 \\
\hline & & & & & & \\
\hline 10 & 51 & 51 & 0.9706 & 0.880 & 1.120 & 0.0100 \\
\hline 12 & 10 & 10 & 0 0105 & 0000 & -----1 & -- \\
\hline 13 & 49 & 49 & 0.9105 & 0.880 & 1.120 & 0.0100 \\
\hline 11 & 43 & 43 & 0.9695 & 0.880 & 1.120 & 0.0100 \\
\hline & & & & & & ------ \\
\hline 40 & 56 & 56 & 0.9500 & 0.880 & 1.120 & 0.0100 \\
\hline 39 & 57 & 57 & 0.9800 & 0.880 & 1.120 & 0.0100 \\
\hline 9 & 55 & 55 & 0.9709 & 0.880 & 1.120 & 0.0100 \\
\hline
\end{tabular}


SUSCEPTÂNCIA EQUIVALENTE DOS BANCOS DE CAPACITORES

\begin{tabular}{|c|c|c|c|}
\hline BARRA & BSHUNT & VALORES & DISCRETOS \\
\hline 18 & 0.100 & $\begin{array}{ll}1 & -> \\
2 & -> \\
3 & -> \\
4 & -> \\
5 & -> \\
6 & -> \\
7 & -> \\
8 & ->\end{array}$ & $\begin{array}{l}0.000 \\
0.050 \\
0.100 * \\
0.120 \\
0.150 \\
0.170 \\
0.220 \\
0.270\end{array}$ \\
\hline 25 & 0.060 & $\begin{array}{ll}1 & -> \\
2 & -> \\
3 & -> \\
4 & -> \\
5 & -> \\
6 & -> \\
7 & -> \\
8 & ->\end{array}$ & $\begin{array}{l}0.000 \\
0.020 \\
0.030 \\
0.040 \\
0.050 \\
0.060 * \\
0.070 \\
0.090\end{array}$ \\
\hline 53 & 0.065 & $\begin{array}{ll}1 & -> \\
2 & -> \\
3 & -> \\
4 & ->\end{array}$ & $\begin{array}{l}0.000 \\
0.065 * \\
0.100 \\
0.165\end{array}$ \\
\hline
\end{tabular}

PARÂMETROS DO ALGORITMO DE DISCRETIZAÇÃO DAS VARIÁVEIS

\begin{tabular}{|c|c|}
\hline TAP: & $\begin{array}{l}\text { gama }=1 \mathrm{e}-06 \\
\text { tau }=1.5\end{array}$ \\
\hline HUNT & $\begin{array}{l}\text { gama }=1 \mathrm{e}-06 \\
\text { tau }=2\end{array}$ \\
\hline & $\begin{array}{l}\text { eta }=1 \\
=0.001\end{array}$ \\
\hline
\end{tabular}




\title{
B.4 Sistema IEEE 118 Barras
}

\author{
CONVERGÊNCIA EM 6 ITERAÇÕES
}

PERDAS $=106.7127 \mathrm{MW}$

ESTADO DO SISTEMA

FLUXOS NOS RAMOS

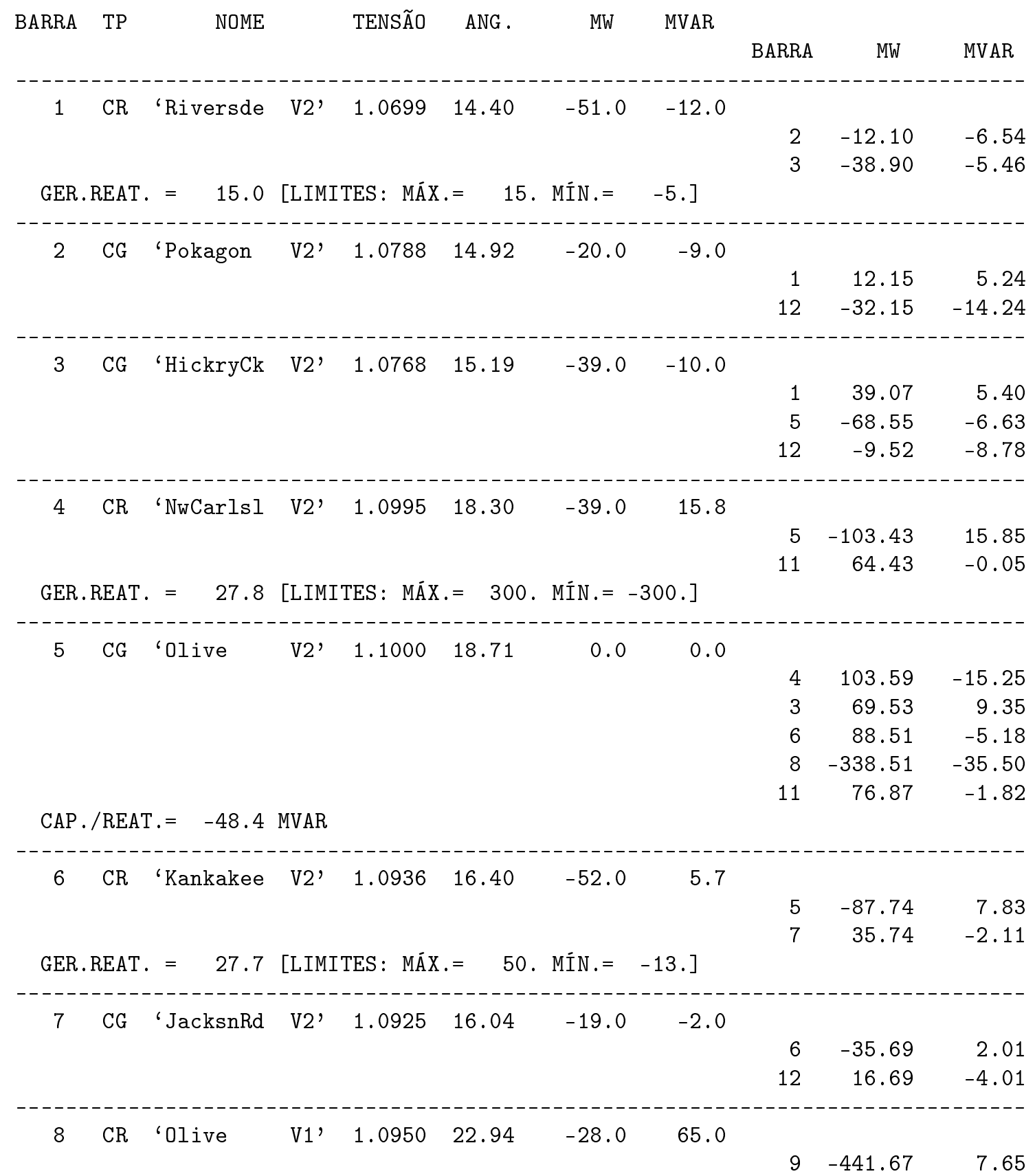




\begin{tabular}{|c|c|c|c|c|c|c|c|c|c|c|}
\hline GEI & REAT & $=65.0$ & [LIM] & TES : MÁX & $=300$ & MÍN. $=$ & 00]$. & $\begin{array}{r}5 \\
30\end{array}$ & $\begin{array}{r}338.51 \\
75.16\end{array}$ & $\begin{array}{l}61.06 \\
-3.74\end{array}$ \\
\hline 9 & CG & 'Bequine & V1, & 1.1000 & 29.42 & 0.0 & 0.0 & $\begin{array}{r}8 \\
10\end{array}$ & $\begin{array}{r}445.68 \\
-445.68\end{array}$ & $\begin{array}{r}-27.55 \\
27.55\end{array}$ \\
\hline GEI & REAT & $\begin{array}{l}\text { 'Breed } \\
=\quad-48.0\end{array}$ & $\begin{array}{l}\text { V1, } \\
\text { [LIM] }\end{array}$ & $\begin{array}{l}1.0994 \\
\text { TES : MÁX }\end{array}$ & $\begin{array}{l}36.31 \\
=\quad 200\end{array}$ & $\begin{array}{l}450.0 \\
\text { MÍN. }=\end{array}$ & $\begin{array}{l}-48.0 \\
47 .]\end{array}$ & 9 & 450.00 & -47.95 \\
\hline 11 & CG & 'SouthBnd & $\mathrm{v} 2$, & 1.0877 & 16.18 & -70.0 & -23.0 & $\begin{array}{r}4 \\
5 \\
12 \\
13\end{array}$ & $\begin{array}{r}-63.72 \\
-75.88 \\
34.28 \\
35.31\end{array}$ & $\begin{array}{r}1.38 \\
4.11 \\
-36.60 \\
8.11\end{array}$ \\
\hline 12 & $\mathrm{CR}$ & 'TwinBrch & $\mathrm{V} 2$ & 1.0924 & 15.75 & 38.0 & 69.8 & $\begin{array}{r}11 \\
2 \\
3 \\
7 \\
14 \\
16 \\
117\end{array}$ & $\begin{array}{r}-34.16 \\
32.35 \\
9.59 \\
-16.67 \\
18.63 \\
8.13 \\
20.13\end{array}$ & $\begin{array}{r}36.72 \\
13.95 \\
6.59 \\
3.57 \\
-1.56 \\
4.13 \\
6.44\end{array}$ \\
\hline
\end{tabular}

GER.REAT. $=79.8$ [LIMITES: MÁX. $=120$. MÍN. $=-35$.

\begin{tabular}{|c|c|c|c|c|c|c|c|c|c|c|}
\hline \multirow[t]{3}{*}{13} & CG & 'Concord & V2, & 1.0749 & 15.01 & -34.0 & -16.0 & & & \\
\hline & & & & & & & & 11 & -35.06 & -8.39 \\
\hline & & & & & & & & 15 & 1.06 & -7.61 \\
\hline \multirow[t]{3}{*}{14} & CG & 'Goshen Jt & V2, & 1.0895 & 15.11 & -14.0 & -1.0 & & & \\
\hline & & & & & & & & 12 & -18.57 & 0.68 \\
\hline & & & & & & & & 15 & 4.57 & -1.68 \\
\hline \multirow[t]{6}{*}{15} & $\mathrm{CR}$ & 'FtWayne & V2, & 1.0873 & 14.67 & -90.0 & -0.0 & & & \\
\hline & & & & & & & & 13 & -1.04 & 4.03 \\
\hline & & & & & & & & 14 & -4.56 & -1.26 \\
\hline & & & & & & & & 17 & -103.97 & 0.55 \\
\hline & & & & & & & & 19 & 11.69 & -2.27 \\
\hline & & & & & & & & 33 & 7.88 & -1.04 \\
\hline
\end{tabular}

GER.REAT. $=30.0$ [LIMITES: MÁX. $=30$. MÍN.$=-10$.

\begin{tabular}{|c|c|c|c|c|c|c|c|c|c|c|}
\hline \multirow[t]{3}{*}{16} & CG & 'N. E. & V2, & 1.0872 & 15.47 & -25.0 & -10.0 & & & \\
\hline & & & & & & & & 12 & -8.11 & -5.34 \\
\hline & & & & & & & & 17 & -16.89 & -4.66 \\
\hline 17 & CG & 'Sorenson & V2, & 1.1000 & 16.86 & -11.0 & -3.0 & & & \\
\hline & & & & & & & & 15 & 105.17 & 0.79 \\
\hline & & & & & & & & 16 & 17.00 & 2.32 \\
\hline & & & & & & & & 18 & 81.46 & -4.35 \\
\hline & & & & & & & & 30 & -233.78 & -1.88 \\
\hline & & & & & & & & 31 & 15.83 & 1.35 \\
\hline & & & & & & & & 113 & 3.32 & -1.23 \\
\hline
\end{tabular}




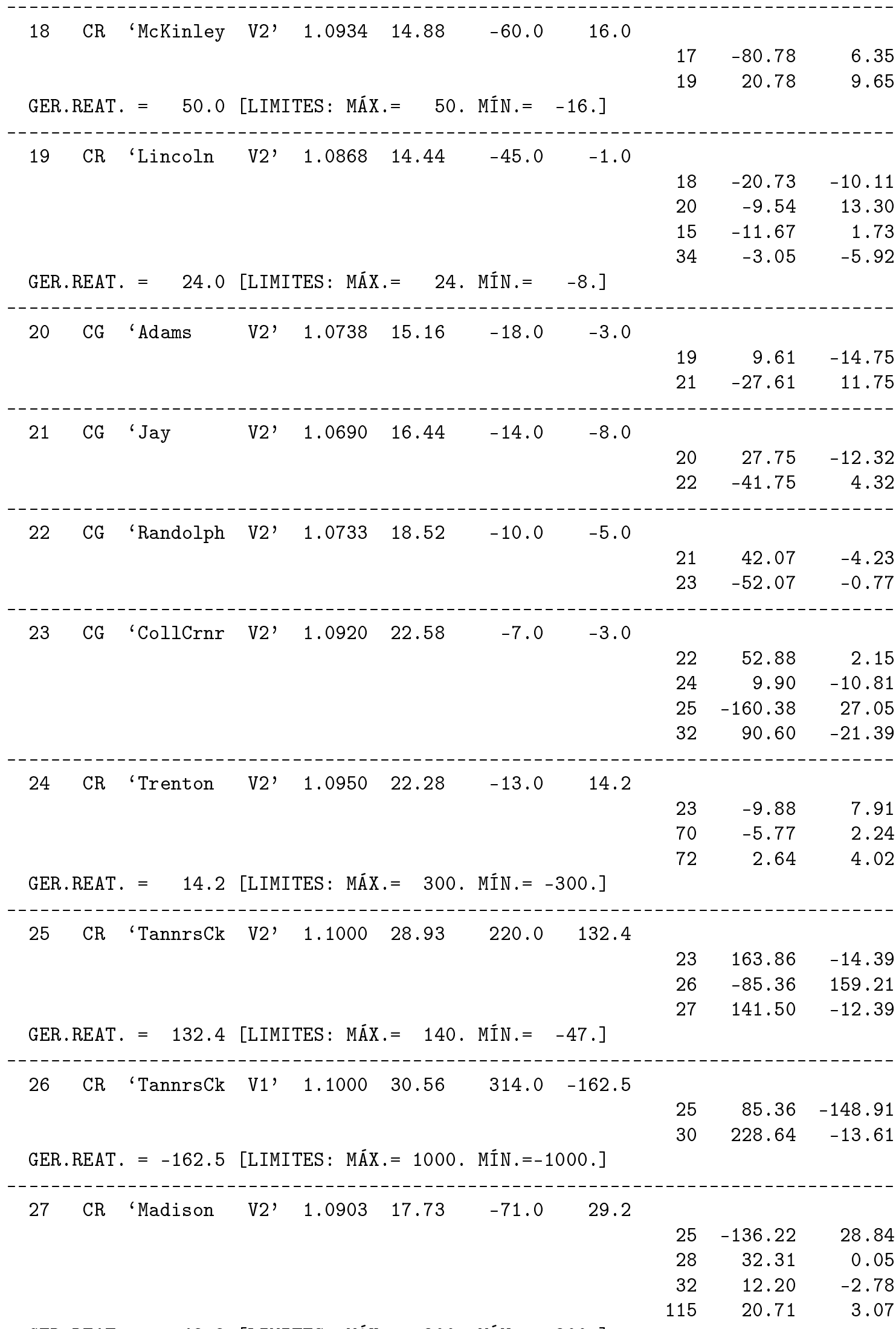

GER.REAT. $=42.2$ [LIMITES: MÁX. $=300$. MÍN. $=-300$. 


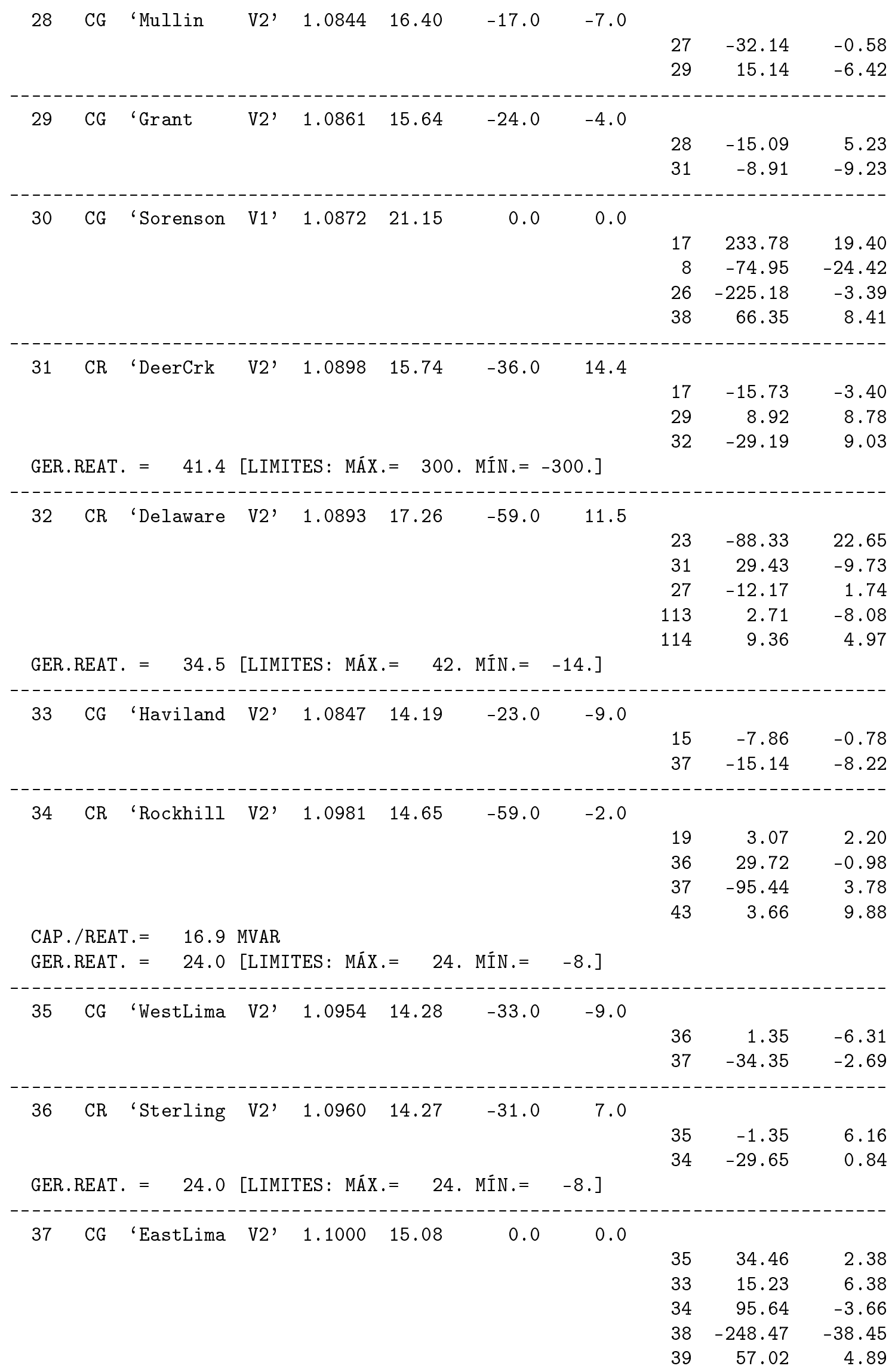




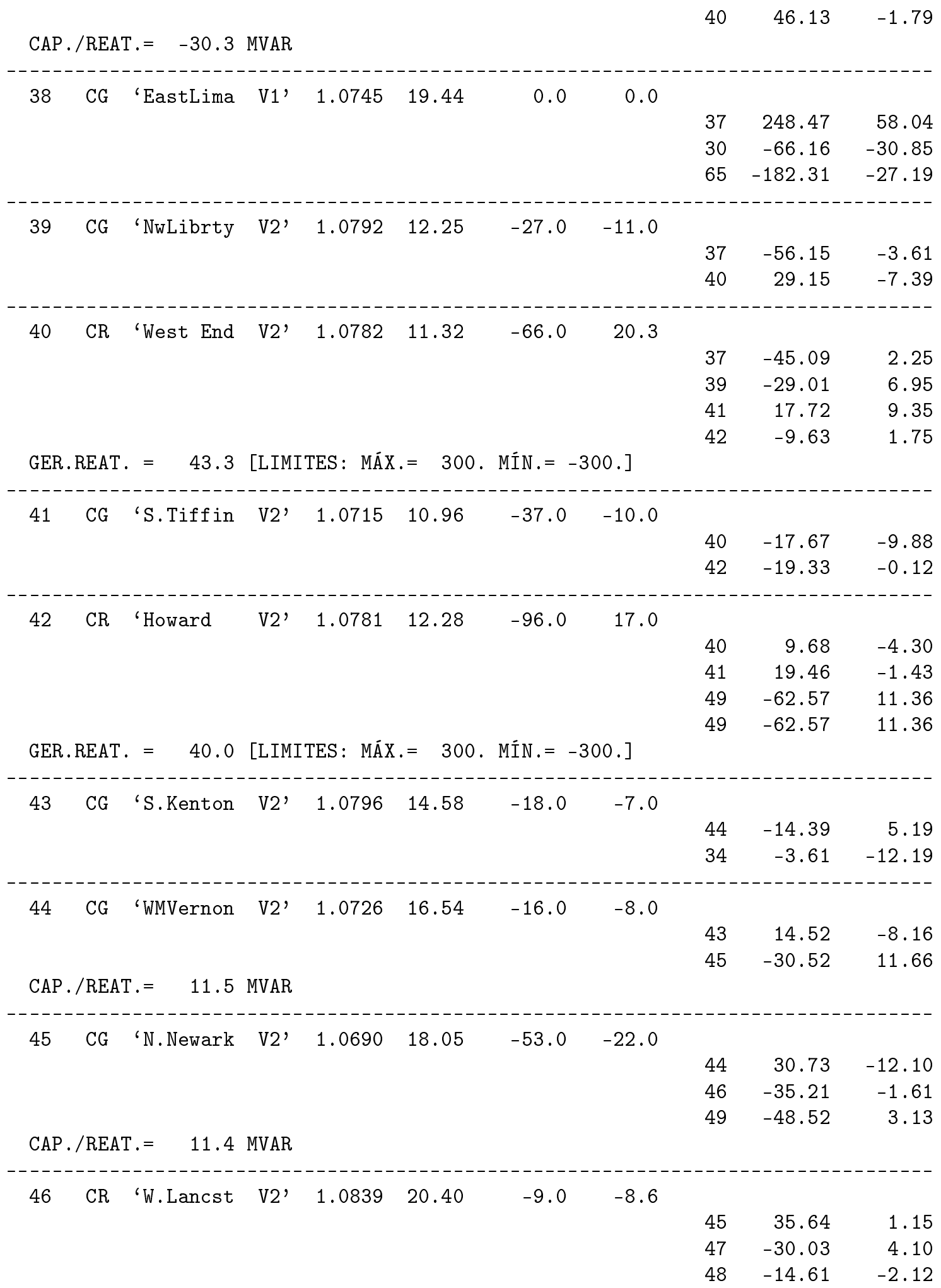

CAP./REAT.$=11.7$ MVAR

GER.REAT. $=1.4$ [LIMITES: MÁX. $=100$. MÍN. $=-100$.

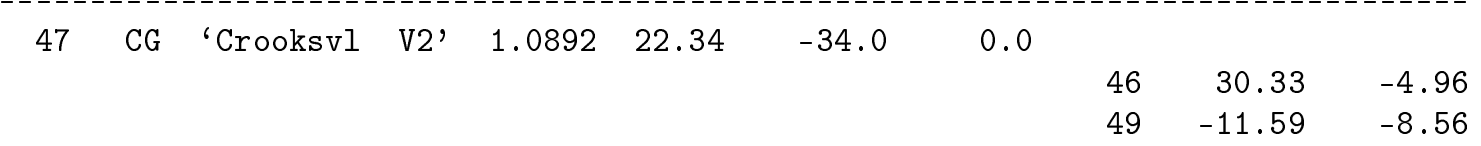




\begin{tabular}{|c|c|c|c|c|c|c|c|c|c|c|}
\hline & & & & & & & & 69 & -52.74 & 13.52 \\
\hline 48 & CG & 'Zanesvll & V2, & 1.0936 & 21.71 & -20.0 & -11.0 & & & \\
\hline & & & & & & & & 46 & 14.72 & \\
\hline & & & & & & & & 49 & -34.72 & \\
\hline CA. & /REA & {$[.=17.9$} & MVAR & & & & & & & \\
\hline 49 & $\mathrm{CR}$ & 'Philo & V2' & 1.0958 & 22.61 & 117.0 & 45.9 & & & \\
\hline & & & & & & & & 47 & 11.62 & \\
\hline & & & & & & & & 42 & 65.10 & \\
\hline & & & & & & & & 42 & 65.10 & -5 . \\
\hline & & & & & & & & 45 & 49.94 & -1 \\
\hline & & & & & & & & 48 & 34.91 & -7 . \\
\hline & & & & & & & & 50 & 50.57 & -0 \\
\hline & & & & & & & & 51 & 62.76 & \\
\hline & & & & & & & & 54 & 36.49 & -2 \\
\hline & & & & & & & & 54 & 35.75 & -4 \\
\hline & & & & & & & & 66 & -125.97 & 25 . \\
\hline & & & & & & & & 66 & -125.97 & 25 . \\
\hline & & & & & & & & 69 & -43.31 & 12 \\
\hline
\end{tabular}

GER.REAT. $=75.9$ [LIMITES: MÁX. $=210$. MÍN. $=-85$.

\begin{tabular}{|c|c|c|c|c|c|c|c|c|c|c|}
\hline 50 & CG & 'WCambrdg & V2, & 1.0844 & 20.77 & -17.0 & -4.0 & $\begin{array}{l}49 \\
57\end{array}$ & $\begin{array}{r}-50.00 \\
33.00\end{array}$ & $\begin{array}{r}1.45 \\
-5.45\end{array}$ \\
\hline 51 & CG & 'Newcmrst & V2, & 1.0664 & 18.48 & -17.0 & -8.0 & $\begin{array}{l}49 \\
52 \\
58\end{array}$ & $\begin{array}{r}-61.16 \\
27.82 \\
16.34\end{array}$ & $\begin{array}{r}0.01 \\
0.89 \\
-8.90\end{array}$ \\
\hline 52 & CG & 'SCoshoct & v2 & 1.0605 & 17.66 & -18.0 & -5.0 & $\begin{array}{l}51 \\
53\end{array}$ & $\begin{array}{r}-27.68 \\
9.68\end{array}$ & $\begin{array}{l}-1.28 \\
-3.72\end{array}$ \\
\hline 53 & CG & 'Wooster & V2, & 1.0609 & 16.80 & -23.0 & -11.0 & $\begin{array}{l}52 \\
54\end{array}$ & $\begin{array}{r}-9.64 \\
-13.36\end{array}$ & $\begin{array}{r}1.58 \\
-12.58\end{array}$ \\
\hline 54 & $\mathrm{CR}$ & 'Torrey & V2, & 1.0777 & 17.47 & -65.0 & 22.9 & $\begin{array}{l}53 \\
49 \\
49 \\
55 \\
56 \\
59\end{array}$ & $\begin{array}{r}13.43 \\
-35.68 \\
-34.82 \\
6.73 \\
17.97 \\
-32.64\end{array}$ & $\begin{array}{r}11.15 \\
1.76 \\
3.21 \\
-0.01 \\
10.87 \\
-4.04\end{array}$ \\
\hline
\end{tabular}

GER.REAT. $=54.9$ [LIMITES: MÁX. $=300$. MÍN. $=-300$.

\begin{tabular}{|c|c|c|c|c|c|c|c|c|c|c|}
\hline 55 & $\mathrm{CR}$ & 'Wagenhls & V2 & 1.0763 & 17.24 & -63.0 & 1.0 & & & \\
\hline & & & & & & & & 54 & -6.73 & -1.14 \\
\hline & & & & & & & & 56 & -19.42 & 6.29 \\
\hline & & & & & & & & 59 & -36.85 & -4.15 \\
\hline
\end{tabular}

GER.REAT. $=23.0$ [LIMITES: MÁX. $=23$. MÍN. $=-8$.

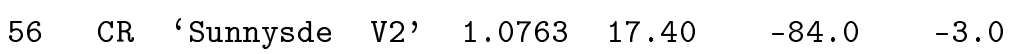




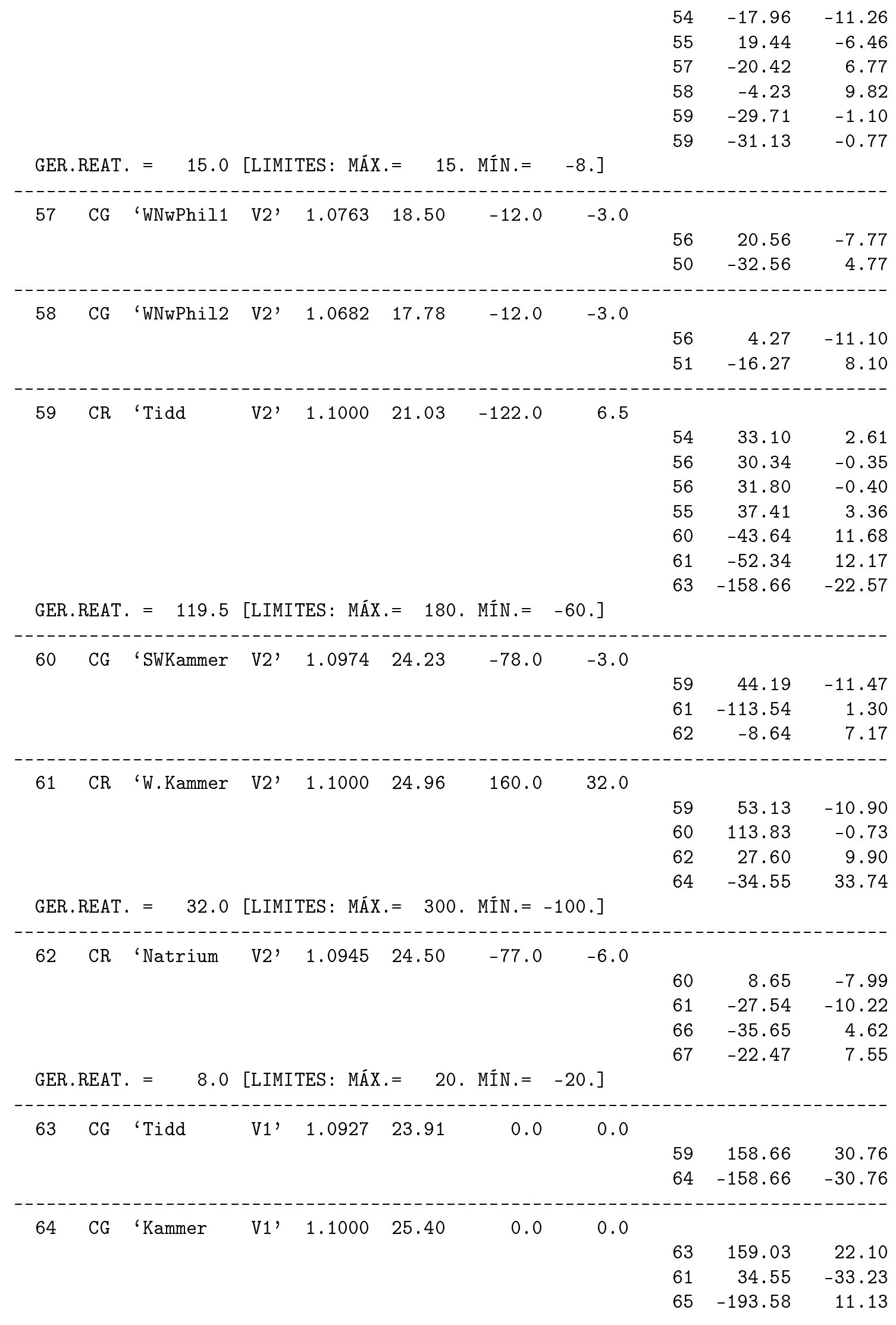

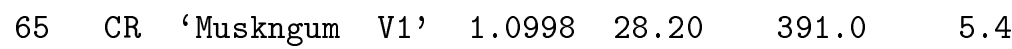




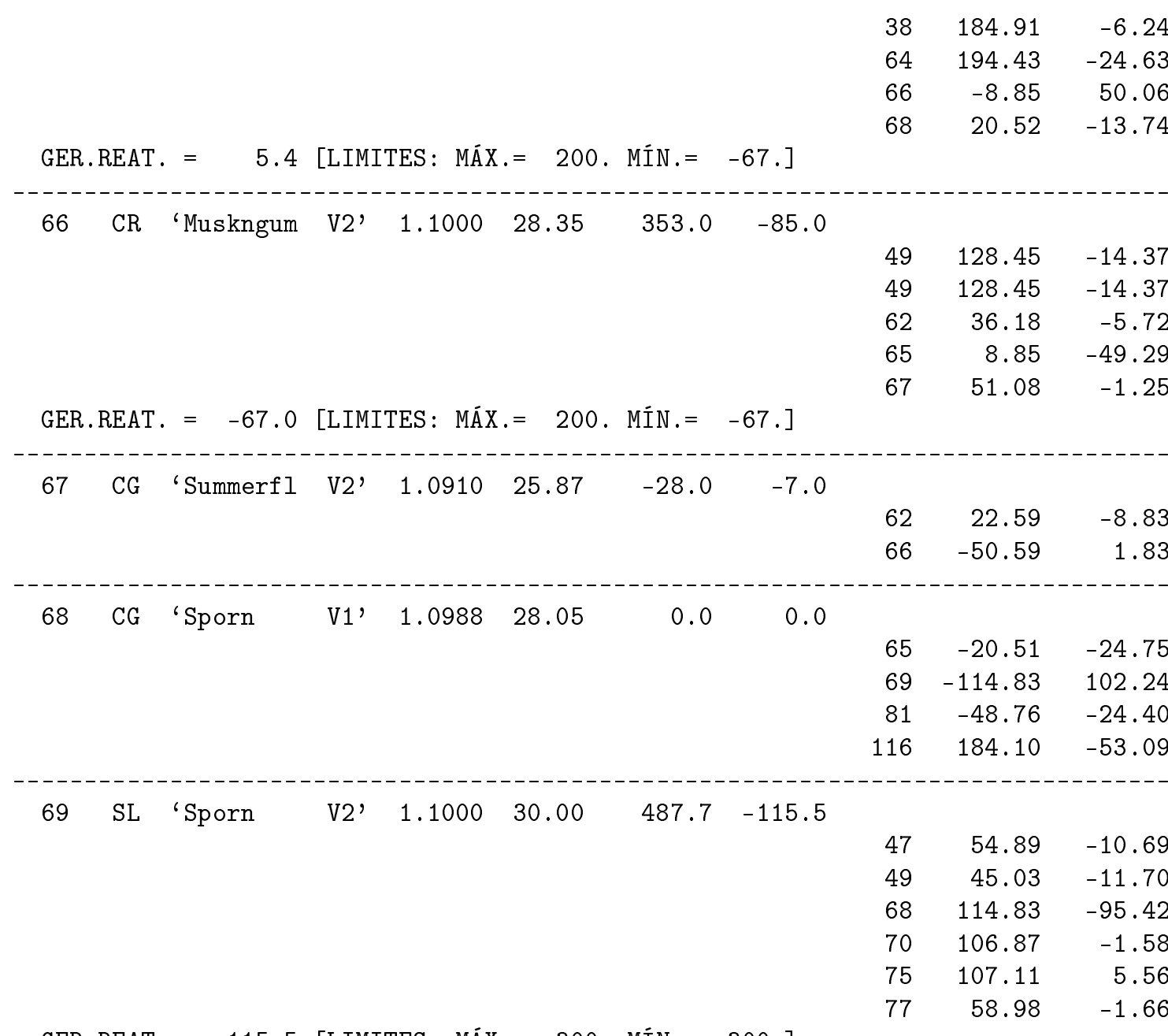

GER.REAT. $=-115.5$ [LIMITES: MÁX.$=300$. MÍN. $=-300$.

$\begin{array}{rlrrrr}70 \quad \mathrm{CR} \text { 'Portsmth V2, } 1.0754 & 23.44 & -66.0 & 12.0 & & \\ & & & 69 & -104.03 & 6.35 \\ & & 24 & 5.77 & -8.04 \\ & & 71 & 15.45 & -11.39 \\ & & 74 & 16.52 & 11.79 \\ & & 75 & 0.30 & 13.29\end{array}$

GER.REAT. $=32.0$ [LIMITES: MÁX. $=32$. MÍN. $=-10$.

\begin{tabular}{|c|c|c|c|c|c|c|c|c|c|c|}
\hline \multirow[t]{4}{*}{71} & CG & 'NPortsmt & V2, & 1.0779 & 23.12 & 0.0 & 0.0 & & & \\
\hline & & & & & & & & 70 & -15.42 & 10.99 \\
\hline & & & & & & & & 72 & 9.42 & -7.22 \\
\hline & & & & & & & & 73 & 6.00 & -3.76 \\
\hline 72 & $\mathrm{CR}$ & 'Hillsbro & V2, & 1.0840 & 22.16 & -12.0 & -2.0 & & & \\
\hline & & & & & & & & 24 & -2.63 & -6.86 \\
\hline & & & & & & & & 71 & -9.37 & 4.82 \\
\hline
\end{tabular}

GER.REAT. $=-2.0$ [LIMITES: MÁX. $=100$. MÍN. $=-100$.

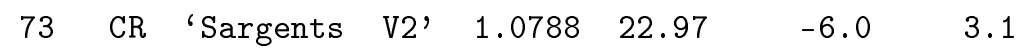

GER.REAT. $=3.1$ [LIMITES: MÁX. $=100$. MÍN. $=-100$. 


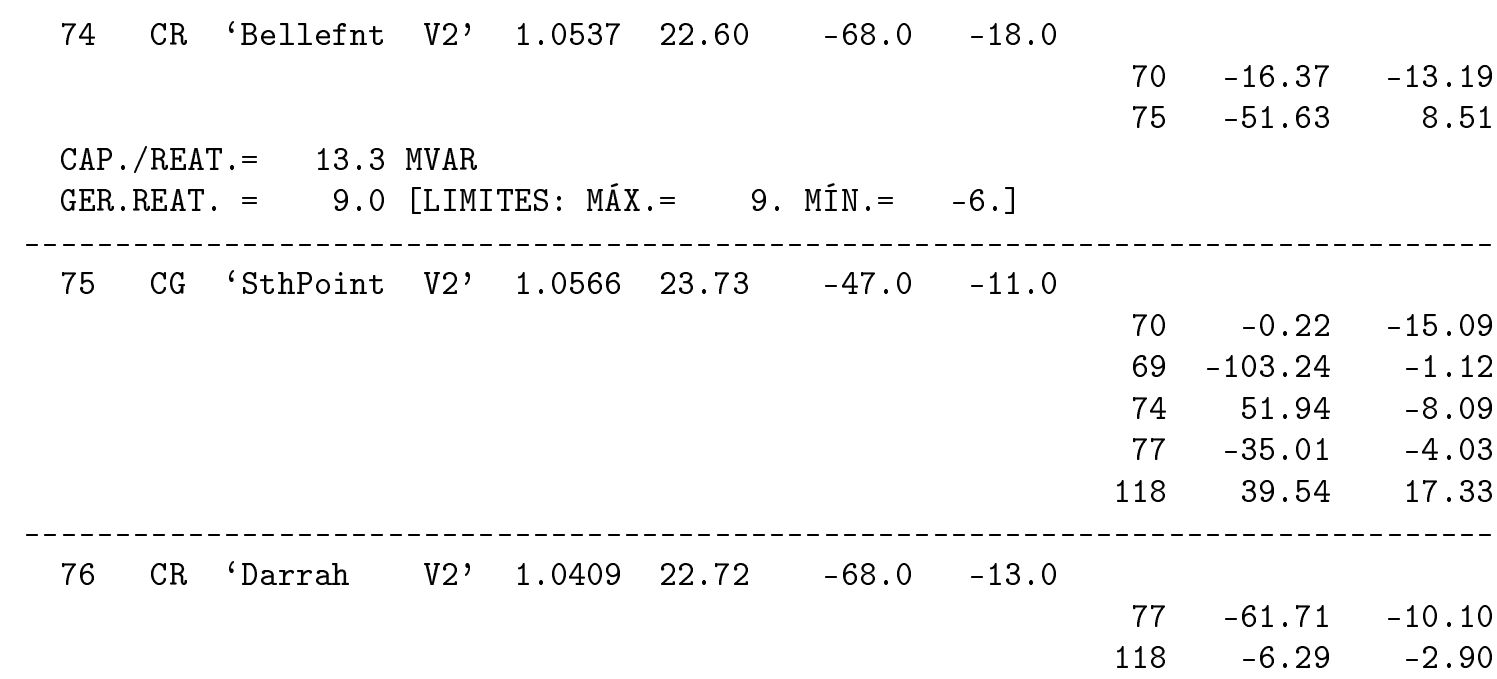

GER.REAT. $=23.0$ [LIMITES: MÁX. $=23$. MÍN. $=-8$.

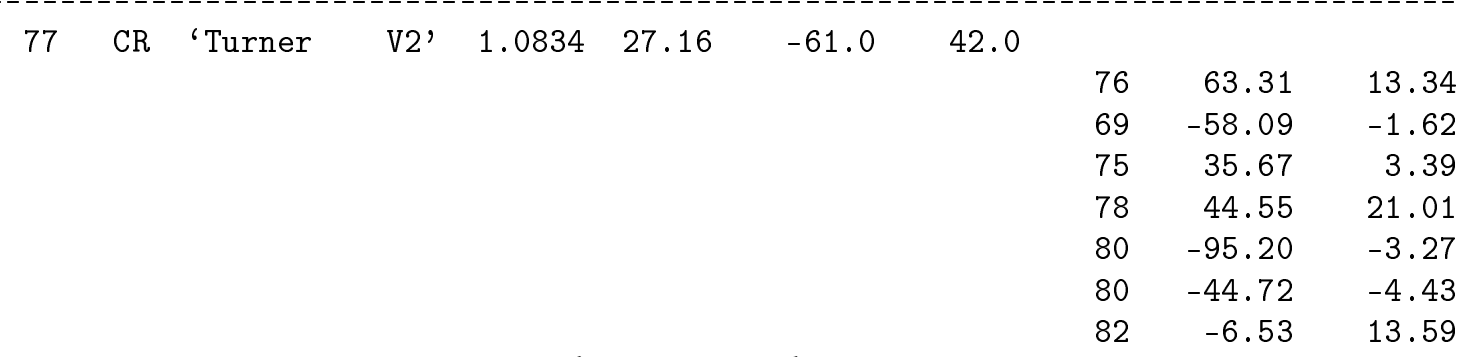

GER.REAT. $=70.0[$ LIMITES: MÁX. $=70$. MÍN. $=-20$.

\begin{tabular}{|c|c|c|c|c|c|c|c|c|c|c|}
\hline 78 & CG & 'Chemical & V2' & 1.0794 & 26.93 & -71.0 & -26.0 & $\begin{array}{l}77 \\
79\end{array}$ & $\begin{array}{l}-44.47 \\
-26.53\end{array}$ & $\begin{array}{r}-21.49 \\
-4.51\end{array}$ \\
\hline CAP & /REA' &.$=23.4$ & MVAR & 1.0818 & 27.23 & -39.0 & -32.0 & $\begin{array}{l}78 \\
80\end{array}$ & $\begin{array}{r}26.56 \\
-65.56\end{array}$ & $\begin{array}{r}4.29 \\
-12.89\end{array}$ \\
\hline GER & $\mathrm{CR}$ & 'CabinCrk & [LIM] & TES : MÁY & $=280$. & MÍN . = & 28.5 & $\begin{array}{l}77 \\
77 \\
79 \\
81 \\
96 \\
97 \\
98 \\
99\end{array}$ & $\begin{array}{l}96.51 \\
45.22 \\
66.15 \\
48.79 \\
18.18 \\
25.55 \\
27.96 \\
18.63\end{array}$ & $\begin{array}{r}4.20 \\
4.87 \\
14.45 \\
-23.12 \\
12.31 \\
16.91 \\
1.85 \\
-2.94\end{array}$ \\
\hline 81 & CG & 'Kanawha & V1, & 1.0996 & 28.51 & 0.0 & 0.0 & $\begin{array}{l}68 \\
80\end{array}$ & $\begin{array}{r}48.79 \\
-48.79\end{array}$ & $\begin{array}{r}-24.01 \\
24.01\end{array}$ \\
\hline 82 & CG & 'Logan & V2 & 1.0727 & 27.67 & -54.0 & -27.0 & $\begin{array}{l}77 \\
83\end{array}$ & $\begin{array}{r}6.60 \\
-49.92\end{array}$ & $\begin{array}{r}-18.12 \\
10.23\end{array}$ \\
\hline
\end{tabular}




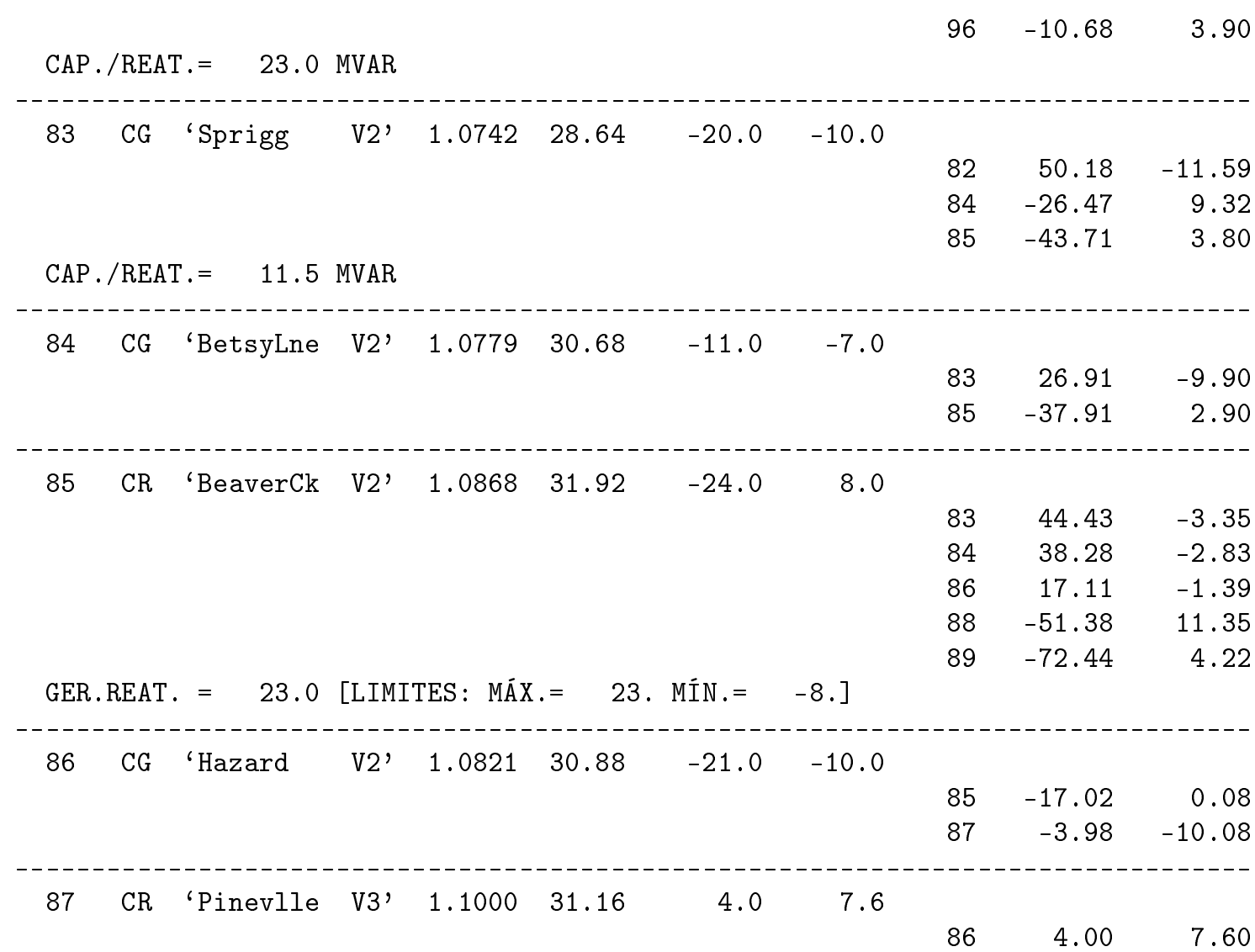

GER.REAT. $=7.6$ [LIMITES: MÁX. $=1000$. MÍN. $=-100$.

\begin{tabular}{|c|c|c|c|c|c|c|c|c|c|c|}
\hline \multirow[t]{3}{*}{88} & CG & 'Fremont & V2, & 1.0860 & 34.58 & -48.0 & -10.0 & & & \\
\hline & & & & & & & & 85 & 51.85 & -10.57 \\
\hline & & & & & & & & 89 & -99.85 & 0.57 \\
\hline \multirow[t]{7}{*}{89} & $\mathrm{CR}$ & 'ClinchRv & v2, & 1.1000 & 38.00 & 607.0 & -17.3 & & & \\
\hline & & & & & & & & 85 & 73.51 & 0.70 \\
\hline & & & & & & & & 88 & 101.02 & 4.29 \\
\hline & & & & & & & & 90 & 57.92 & -5.22 \\
\hline & & & & & & & & 90 & 110.36 & -6.30 \\
\hline & & & & & & & & 92 & 200.86 & -5.19 \\
\hline & & & & & & & & 92 & 63.31 & -5.54 \\
\hline
\end{tabular}

GER.REAT. $=-17.3$ [LIMITES: MÁX. $=300$. MÍN. $=-210$.

$\begin{array}{rrrrrrr}90 \quad \mathrm{CR} \text { 'Holston V2' } 1.0836 & 32.67 & -163.0 & 10.8 & & & \\ & & & & & -56.48 & 7.31 \\ & & & & 89 & -107.97 & 10.02 \\ & & & 91 & 1.45 & -6.58\end{array}$

GER.REAT. $=52.8$ [LIMITES: MÁX. $=300$. MÍN. $=-300$.

\begin{tabular}{|c|c|c|c|c|c|c|c|c|c|c|}
\hline 91 & CR & 'HolstonT & V2, & 1.0879 & 32.54 & -10.0 & 7.4 & & & \\
\hline & & & & & & & & 90 & $\begin{array}{l}-1.44 \\
-8.56\end{array}$ & 5.34 \\
\hline
\end{tabular}

GER.REAT. $=7.4$ [LIMITES: MÁX. $=100$. MÍN. $=-100$.

$92 \mathrm{CR}$ 'Saltvlle V2' $1.0875 \quad 33.12 \quad-65.0 \quad-1.0$ 


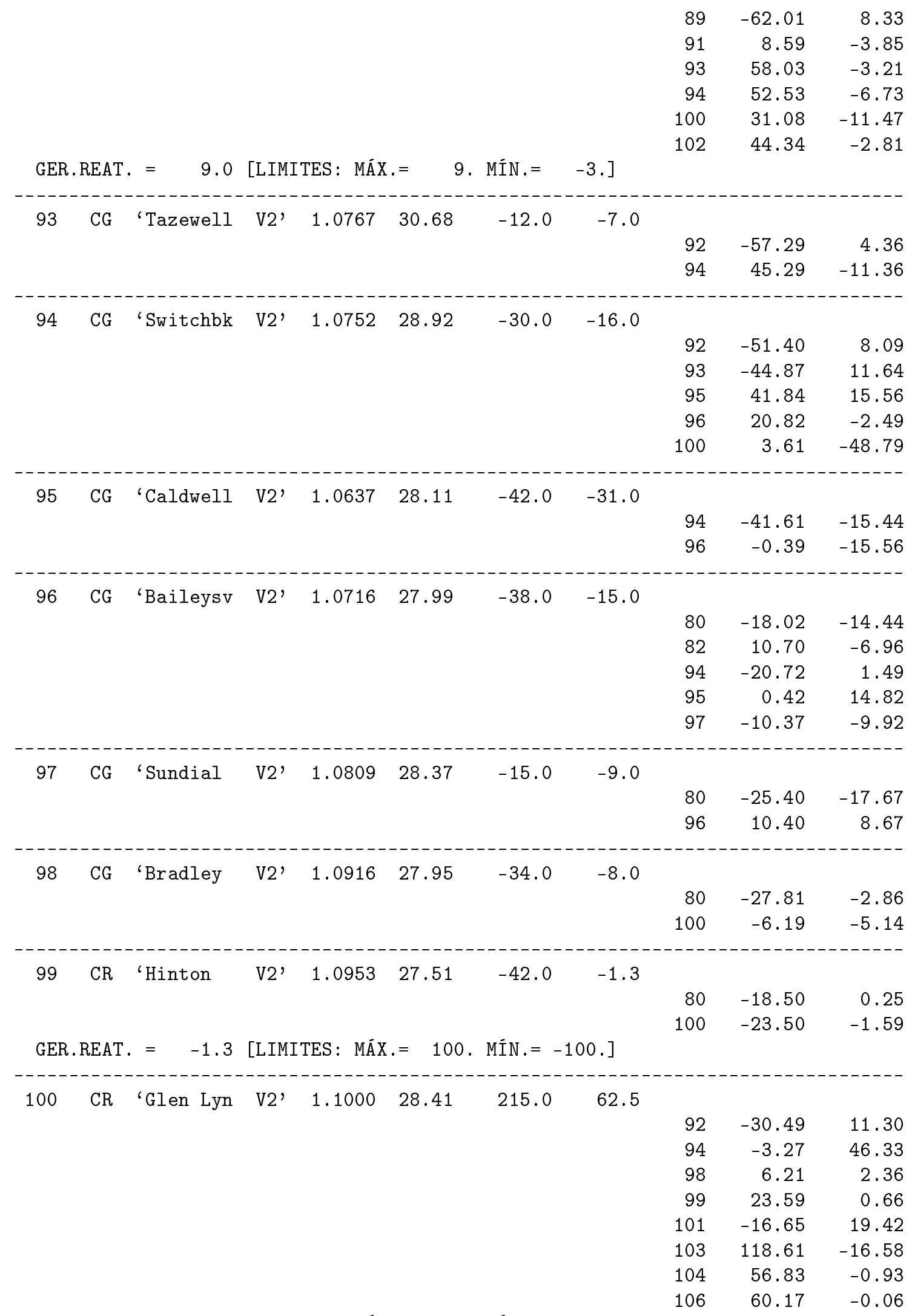

GER.REAT. $=80.5$ [LIMITES: MÁX. $=155$. MÍN. $=-50$.

101 CG 'Wythe V2' $1.0810 \quad 29.69 \quad-22.0 \quad-15.0$ 


\begin{tabular}{|c|c|c|c|c|c|c|c|c|c|c|}
\hline & & & & & & & & $\begin{array}{l}100 \\
102\end{array}$ & $\begin{array}{r}16.81 \\
-38.81\end{array}$ & $\begin{array}{r}-20.65 \\
5.65\end{array}$ \\
\hline \multirow[t]{3}{*}{102} & CG & 'Smythe & V2, & 1.0839 & 31.90 & -5.0 & -3.0 & & & \\
\hline & & & & & & & & 92 & -44.14 & 2.89 \\
\hline & & & & & & & & 101 & 39.14 & -5.89 \\
\hline \multirow[t]{5}{*}{103} & $\mathrm{CR}$ & 'Claytor & V2, & 1.0915 & 25.32 & 17.0 & 11.8 & & & \\
\hline & & & & & & & & 100 & -116.72 & 19.57 \\
\hline & & & & & & & & 104 & 32.06 & -2.39 \\
\hline & & & & & & & & 105 & 42.24 & -4.97 \\
\hline & & & & & & & & 110 & 59.41 & -0.40 \\
\hline
\end{tabular}

GER.REAT. $=27.8$ [LIMITES: MÁX. $=40$. MÍN. $=-15$.

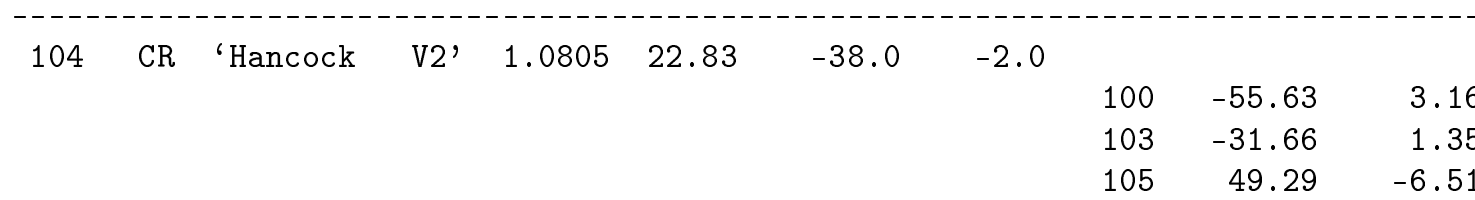

GER.REAT. $=23.0$ [LIMITES: MÁX. $=23$. MÍN. $=-8$.

$\begin{array}{rrrrrr}105 \text { CR 'Roanoke V2' } 1.078321 .88 & -31.0 & -3.0 & & & \\ & & & -41.43 & 5.02 \\ & & 104 & -49.08 & 6.74 \\ & & 106 & 8.54 & 13.07 \\ & & 107 & 26.72 & -1.57 \\ & & 108 & 24.25 & -3.01\end{array}$

CAP. $/$ REAT. $=23.3$ MVAR

GER.REAT. $=23.0$ [LIMITES: MÁX. $=23$. MÍN. $=-8$.

\begin{tabular}{|c|c|c|c|c|c|c|c|c|c|c|}
\hline \multirow[t]{4}{*}{106} & CG & 'Cloverdl & v2, & 1.0704 & 21.74 & -43.0 & -16.0 & & & \\
\hline & & & & & & & & 100 & -58.36 & 3.2 \\
\hline & & & & & & & & 105 & -8.51 & -13.78 \\
\hline & & & & & & & & 107 & 23.88 & -5.4 \\
\hline \multirow[t]{3}{*}{107} & $\mathrm{CR}$ & 'Reusens & V2, & 1.0665 & 19.44 & -50.0 & -3.1 & & & \\
\hline & & & & & & & & 105 & -26.39 & \\
\hline & & & & & & & & 106 & -23.61 & \\
\hline
\end{tabular}
CAP. $/$ REAT . $=$
6.8 MVAR
GER.REAT. =
8.9 [LIMITES: MÁX.$=200$. MÍN.$=-200$.

\begin{tabular}{|c|c|c|c|c|c|c|c|c|c|c|}
\hline \multirow[t]{3}{*}{108} & CG & 'Blaine & V2, & 1.0742 & 21.01 & -2.0 & -1.0 & \multirow{3}{*}{$\begin{array}{l}105 \\
109\end{array}$} & \multirow{3}{*}{$\begin{array}{r}-24.12 \\
22.12\end{array}$} & \multirow{3}{*}{$\begin{array}{r}2.30 \\
-3.30\end{array}$} \\
\hline & & & & & & & & & & \\
\hline & & & & & & & & & & \\
\hline \multirow[t]{3}{*}{109} & CG & 'Franklin & V2 & 1.0729 & 20.67 & -8.0 & -3.0 & & & \\
\hline & & & & & & & & 108 & -22.07 & 2.99 \\
\hline & & & & & & & & 110 & 14.07 & -5.99 \\
\hline \multirow[t]{5}{*}{110} & $\mathrm{CR}$ & 'Fieldale & v2, & 1.0731 & 20.06 & -39.0 & -7.0 & & & \\
\hline & & & & & & & & 103 & -58.25 & 3.07 \\
\hline & & & & & & & & 109 & -14.02 & 4.97 \\
\hline & & & & & & & & 111 & -35.75 & -3.69 \\
\hline & & & & & & & & 112 & 69.02 & -4.44 \\
\hline
\end{tabular}

CAP./REAT. $=6.9$ MVAR

GER.REAT. $=23.0$ [LIMITES: MÁX. $=23$. MÍN. $=-8$. 


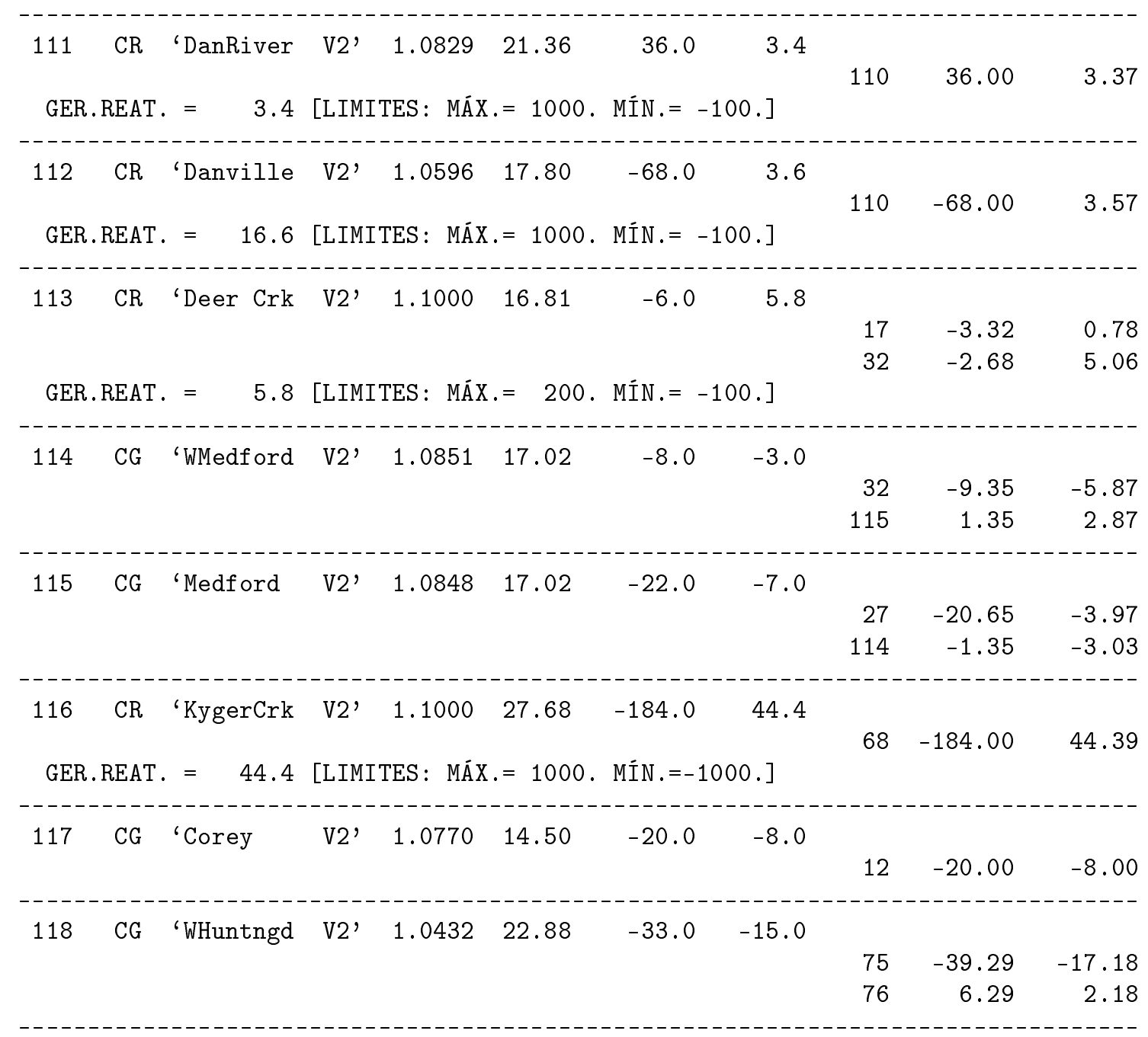

TAPS DOS TRANSFORMADORES

\begin{tabular}{|c|c|c|c|c|c|c|}
\hline BARRA & BARRA & BARRA & TAP & TAP & TAP & PASSO \\
\hline INICIAL & FINAL & CRTL. & & MÍN. & MÁX. & DISCRETO \\
\hline-- & -- & -- & & $-1--$ & ----- & -------- \\
\hline 8 & 5 & 5 & 0.9850 & 0.880 & 1.120 & 0.0075 \\
\hline 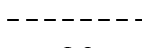 & -- & --1 & ------ & ---- & ---- & -------- \\
\hline 26 & 25 & 25 & 1.0525 & 0.880 & 1.120 & 0.0075 \\
\hline-- & --- & --- & ----- & ------- & ---- & -------- \\
\hline 30 & 17 & 17 & 0.9850 & 0.880 & 1.120 & 0.0075 \\
\hline- & --- & --- & ------- & ------- & ----- & --------- \\
\hline 38 & 37 & 37 & 0.9625 & 0.880 & 1.120 & 0.0075 \\
\hline & --- & ---- & ------- & ------- & ----- & --------- \\
\hline 63 & 59 & 59 & 0.9850 & 0.880 & 1.120 & 0.0075 \\
\hline & -- & --1 & ------- & ------1 & ----- & --------- \\
\hline 64 & 61 & 61 & 1.0075 & 0.880 & 1.120 & 0.0075 \\
\hline & & -- & ----- & ------ & ----- & --------- \\
\hline 65 & 66 & 66 & 0.9850 & 0.880 & 1.120 & 0.0075 \\
\hline & & -- & - & - & ---- & -------- \\
\hline 68 & 69 & 69 & 0.9700 & 0.880 & 1.120 & 0.0075 \\
\hline
\end{tabular}




$\begin{array}{lllllll}81 & 80 & 80 & 0.9925 & 0.880 & 1.120 & 0.0075\end{array}$

SUSCEPTÂNCIA EQUIVALENTE DOS BANCOS DE CAPACITORES

\begin{tabular}{|c|c|c|c|}
\hline BARRA & BSHUNT & VALORES & DISCRETOS \\
\hline & & -----1 & $--7+7>$ \\
\hline 5 & -0.400 & $1->$ & 0.000 \\
\hline & & $2->$ & $-0.400 *$ \\
\hline & & ----- & ------ \\
\hline 34 & 0.140 & $1->$ & 0.000 \\
\hline & & $2->$ & 0.060 \\
\hline & & $3->$ & 0.070 \\
\hline & & $4->$ & 0.130 \\
\hline & & $5->$ & $0.140 *$ \\
\hline & & $6->$ & 0.200 \\
\hline-- & ----- & ----- & -------- \\
\hline 37 & -0.250 & $1->$ & 0.000 \\
\hline & & $2->$ & $-0.250 *$ \\
\hline-- & & ----- & ------ \\
\hline 44 & 0.100 & $1->$ & 0.000 \\
\hline & & $2->$ & $0.100 *$ \\
\hline--- & ------- & ----- & -------- \\
\hline 45 & 0.100 & $1->$ & 0.000 \\
\hline & & $2->$ & $0.100 *$ \\
\hline - & ----- & ----- & -------- \\
\hline 46 & 0.100 & $1->$ & 0.000 \\
\hline & & $2->$ & $0.100 *$ \\
\hline & $-\ldots-\ldots$ & ---- & -------- \\
\hline 48 & 0.150 & $1->$ & 0.000 \\
\hline & & $2->$ & $0.150 *$ \\
\hline & ------ & ----- & -------- \\
\hline 74 & 0.120 & $1->$ & 0.000 \\
\hline & & $2->$ & 0.080 \\
\hline & & $3->$ & $0.120 *$ \\
\hline & & $4->$ & 0.200 \\
\hline & $\ldots$ & --- & ------- \\
\hline 79 & 0.200 & $1->$ & 0.000 \\
\hline & & $2->$ & 0.100 \\
\hline & & $3->$ & $0.200 *$ \\
\hline & & --- & -------- \\
\hline 82 & 0.200 & $1->$ & 0.000 \\
\hline & & $2->$ & 0.100 \\
\hline & & $3->$ & $0.200 *$ \\
\hline 83 & 0.100 & $1->$ & 0.000 \\
\hline & & $2->$ & $0.100 *$ \\
\hline & & $3->$ & 0.200 \\
\hline 105 & 0.200 & $1->$ & 0.000 \\
\hline & & $2->$ & 0.100 \\
\hline & & $3->$ & $0.200 *$ \\
\hline
\end{tabular}




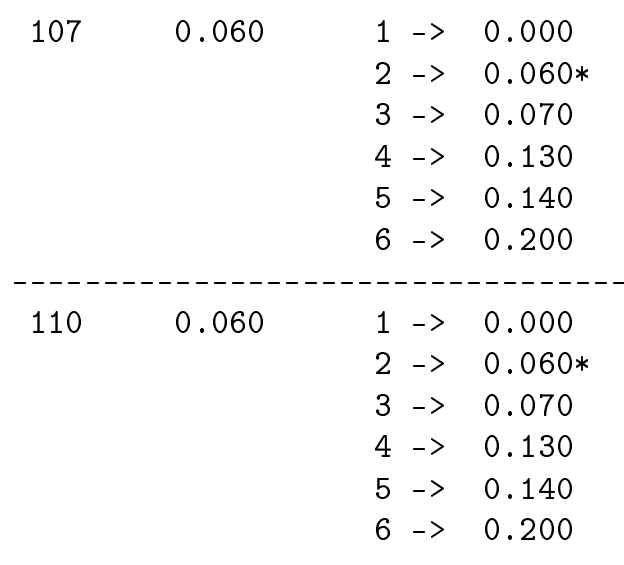

PARÂMETROS DO ALGORITMO DE DISCRETIZAÇÃO DAS VARIÁVEIS

\begin{tabular}{|c|c|}
\hline TAP: & $\begin{array}{l}\text { gama }=1 \mathrm{e}-05 \\
\text { tau }=10\end{array}$ \\
\hline BSHUNT : & $\begin{aligned} \text { gama } & =1 e-05 \\
\text { tau } & =10\end{aligned}$ \\
\hline & $\begin{array}{l}\text { eta }=1 \\
=0.0001\end{array}$ \\
\hline
\end{tabular}

